\title{
Thermodynamical Formalism and Multifractal Analysis for Meromorphic Functions of finite order
}

\author{
Volker Mayer \\ Mariusz Urbański
}

Volker Mayer, Université de Lille I, UFR de Mathématiques, UMR 8524 du CNRS, 59655 Villeneuve D'AscQ Cedex, France

E-mail address: volker.mayer@math.univ-lille1.fr

Web: math.univ-lille1.fr/ mayer

Mariusz Urbański, Department of Mathematics, University of North Texas, Denton, TX 76203-1430, USA

E-mail address: urbanski@unt.edu

Web: www.math.unt.edu/ urbanski 
Received by editor December 21, 2006.

2000 Mathematics Subject Classification. Primary 30D05, 37F10

Key words and phrases. Holomorphic dynamics, Thermodynamical Formalism,

Transcendental functions, Fractal geometry.

Research of the second author is supported in part by the NSF Grant DMS

0400481.

Abstract. The thermodynamical formalism has been developed in MyU2

for a very general class of transcendental meromorphic functions. A function $f: \mathbb{C} \rightarrow \hat{\mathbb{C}}$ of this class is called dynamically (semi-) regular. The key point in MyU2 was that one worked with a well chosen Riemannian metric space $(\hat{\mathbb{C}}, \sigma)$ and that the Nevanlinna theory was employed.

In the present manuscript we first improve $\mathbf{M y U 2}$ in providing a systematic account of the thermodynamical formalism for such a meromorphic function $f$ and all potentials that are Hölder perturbations of $-t \log \left|f^{\prime}\right|_{\sigma}$. In this general setting, we prove the variational principle, we show the existence and uniqueness of Gibbs states (with the definition appropriately adapted for the transcendental case) and equilibrium states of such potentials, and we demonstrate that they coincide. There is also given a detailed description of spectral and asymptotic properties (spectral gap, Ionescu-Tulcea and Marinescu Inequality) of Perron-Frobenius operators, and their stochastic consequences such as the Central Limit Theorem, K-mixing, and exponential decay of correlations.

Then we provide various, mainly geometric, applications of this theory. Indeed, we examine the finer fractal structure of the radial (in fact nonescaping) Julia set by developing the multifractal analysis of Gibbs states. In particular, the Bowen's formula for the Hausdorff dimension of the radial Julia set from MyU2 is reproved. Moreover, the multifractal spectrum function is proved to be convex, real-analytic and to be the Legendre transform conjugate to the temperature function. In the last chapter we went even further by showing that, for a analytic family satisfying a symmetric version of the growth condition (1.1) in a uniform way, the multifractal spectrum function is real-analytic also with respect to the parameter. Such a fact, up to our knowledge, has not been so far proved even for hyperbolic rational functions nor even for the quadratic family $z \mapsto z^{2}+c$. As a by-product of our considerations we obtain real analyticity of the Hausdorff dimension function. 


\section{Contents}

Chapter 1. Introduction

Chapter 2. Balanced functions

Chapter 3. Transfer operator and Nevanlinna Theory 15

3.1. Choice of a Riemannian metric and transfer operator $\quad 15$

3.2. Nevanlinna Theory and Borel Sums 16

Chapter 4. Preliminaries, Hyperbolicity and Distortion Properties. 21

4.1. Dynamical preliminaries and hyperbolicity 21

4.2. Distortion properties 24

4.3. Hölder functions and dynamical Hölder property 25

Chapter 5. Perron-Frobenius Operators and Generalized Conformal Measures 27 5.1. Tame potentials 27

5.2. Growth condition and cohomological Perron-Frobenius operator 28

5.3. Topological pressure and existence of conformal measures

5.4. Thermodynamical Formalism $\quad 32$

5.5. The support and uniqueness of the conformal measure 35

Chapter 6. Finer properties of Gibbs States 39

6.1. The two norm inequality and the spectral gap 39

6.2. Ergodic properties of Gibbs States 43

6.3. Decay of correlations and Central Limit Theorem

6.4. Cohomologies and $\sigma^{2}=0 \quad 49$

6.5. Variational Principle 55

Chapter 7. Regularity of Perron-Frobenius Operators and Topological Pressure

7.1. Analyticity of Perron-Frobenius Operators 59

7.2. Analyticity of pressure $\quad 62$

7.3. Derivatives of the Pressure function 64

Chapter 8. Multifractal analysis 83

8.1. Hausdorff dimension of Gibbs states 83 
8.2. The temperature function 86

8.3. Multifractal analysis

Chapter 9. Multifractal Analysis of Analytic Families of Dynamically Regular Functions

9.1. Extensions of Harmonic Functions

9.2. Holomorphic Families and Quasi-Conformal Conjugacies

9.3. Real Analyticity of the Multifractal Function 


\section{CHAPTER 1}

\section{Introduction}

The thermodynamic formalism of hyperbolic (expanding) rational functions and Hölder continuous potentials on the Riemann sphere is by now fairly well developed and understood. Being a part of a more general theory of distance expanding maps, its systematic account can be found in [PU] (see also [Zin]). It was greatly influenced by the work Bw1 of R. Bowen, R1 of D. Ruelle and others. In particular, the topological pressure was introduced and analyzed, the spectral and asymptotic properties of Perron-Frobenius operators are established, regularity properties (real analyticity) of topological pressure function are also established, Gibbs and equilibrium states are shown to exist, to coincide and to be unique. Moreover, the resulting dynamical systems are "strongly" mixing (K-mixing, weak Bernoulli), satisfy the Central Limit Theorem, exponential decay of correlations, and the Invariant Principle Almost Surely.

The fractal geometry of hyperbolic rational functions also seems to have reached its maturity period. As far as we know, its modern development began with the work $\mathbf{B w 2}$ of R. Bowen, paralleled by Sullivan's activity (see $[\mathbf{S u}]$ ), and followed by PUZ. It is known that for hyperbolic rational functions $\operatorname{HD}(\mathcal{J}(f))$, the Hausdorff dimension of the Julia set is given by Bowen's formula (the zero of the pressure function), the conformal measure is unique (and its exponent equals to $\operatorname{HD}(\mathcal{J}(f))$ ), both Hausdorff and packing measures are positive and finite and coincide up to a multiplicative constant. Also the Hausdorff dimension of the Julia set is shown to depend analytically on the parameter $([\mathbf{R 2}])$ and the multifractal formalism, analyzing the structure of the level sets of the local dimension function of a given Gibbs measure, is developed. Actually all of this can be found with proofs in [PU; the survey article [Ur1 briefly summarizes hyperbolic rational functions and deals in greater detail with parabolic and non-recurrent rational functions.

Notice that all of this is quite different for transcendental functions. As we will see, there is a Bowen's formula but the zero of the pressure is not the Hausdorff dimension of the Julia set. It determines the hyperbolic dimension which equals to the Hausdorff dimension of $\mathcal{J}_{r}(f)$, the radial (or conical) Julia set of the function $f$. Such a Bowen's formula has been established in UZ1 for hyperbolic exponential functions. As a corollary the authors obtained that there is a gap between the hyperbolic dimension and the Hausdorff dimension of the Julia set itself (the later being of dimension two, a result from McMullen's paper [McM]). Such a phenomenon is in big contrast to what happens for rational functions. That transcendental functions can have such a gap has been observed for the first time (in terms of the critical Poincaré exponent) by G. Stallard [St1. 1] Finally, the behavior of

\footnotetext{
${ }^{1}$ We like to thank L. Rempe for bringing this result to our attention.
} 
Hausdorff and packing measures has been studied in MyU3 (see also [UZ1]). It turned out that the Hausdorff measure (of the radial Julia set) may vanish whereas the packing measure may happen to be locally infinite.

There is yet one more important direction of research concerning fractal geometry of Julia sets of transcendental functions. It copes with determining precise values and estimates of the Hausdorff dimension of Julia sets. G. Stallard has done a lot in this direction (see the survey [St2]), there are also contributions of J. Kotus, B. Karpińska, P. Rippon and the authors of this memoir. We essentially do not touch this topic here. The reader interested in the early historical development of the "measurable" (thermodynamic formalism, fractal geometry, absolutely continuous invariant measures) theory of transcendental functions, can find some useful information in [KU4].

The present work exclusively concerns transcendental dynamics. The first work on thermodynamical formalism is $\mathbf{B a}$ where Barański was dealing with the tangent family. Expanding the ideas from Ba led to [KU1, where Walters expanding maps and Barański maps were introduced and studied. One important feature of maps treated in $\mathbf{B a}$ and $[\mathbf{K U 1}$ was that all analytic inverse branches were welldefined at all points of Julia sets. This property dramatically fails for example for such classical functions as $f_{\lambda}(z)=\lambda e^{z}$ (there are no well-defined inverse branches at infinity) and the Perron-Frobenius operator, taken in its most natural sense, is even not well-defined:

$$
\mathcal{L}_{t} \mathbb{1}(w)=\sum_{z \in f_{\lambda}^{-1}(w)}\left|f_{\lambda}^{\prime}(z)\right|^{-t}=\sum_{z \in f_{\lambda}^{-1}(w)}|z|^{-t}=+\infty .
$$

To remedy this situation, the periodicity of $f_{\lambda}$ was exploited to project the dynamics of these functions down to the cylinder and the appropriate thermodynamical formalism was developed in UZ1 and UZ2. This approach has been adopted to other periodic transcendental functions (besides the papers cited above, see also CS1, CS2, KU2, MyU1, UZ3, UZ4 and the survey [KU4]).

The situation changed completely with the new approach from MyU2. It allows to handle all the periodic functions cited above in a uniform way and, most importantly, it goes much farther beyond. The key point of $\mathbf{M y U 2}$ is to associate to a given transcendental function $f: \mathbb{C} \rightarrow \hat{\mathbb{C}}$ a Riemannian metric $\sigma$ which then allows to perform, with the help of Nevanlinna theory, the whole thermodynamical formalism for the potentials of the form

$$
-t \log \left|f^{\prime}\right|_{\sigma}
$$

$\left|f^{\prime}\right|_{\sigma}$ being the derivative of $f$ with respect to the metric $\sigma$. This approach applies to any finite order meromorphic function $f$ that satisfies a growth condition for the derivative of the form

$$
\left|f^{\prime}(z)\right| \geq \kappa^{-1}(1+|z|)^{\alpha_{1}}\left(1+|f(z)|^{\alpha_{2}}, \quad z \in \mathcal{J}(f) \backslash f^{-1}(\{\infty\})\right.
$$

where $\kappa>0$ and where $\underline{\alpha}_{2}>\max \left\{-\alpha_{1}, 0\right\}$. Such a function will be called dynamically semi-regular if it is in addition hyperbolic (precise definitions are given in Chapters 2 and 4). Notice that this growth condition is quite natural for many transcendental functions. Besides the periodic (tangent, sine and exponential as well as elliptic) functions also their composition with polynomials and many other functions 
like the cosine-root family and functions with polynomial or rational Schwarzian derivative share this property.

In the present paper we provide a systematic account of the thermodynamic formalism for dynamically regular functions and tame potentials, i.e. potentials of the form

$$
-t \log \left|f^{\prime}\right|_{\sigma}+h, \quad \text { where } t>\rho / \alpha
$$

( $\rho$ being the order of the transcendental function $f$ and $\alpha=\alpha_{1}+\underline{\alpha}_{2}$ coming from the derivative growth condition) and $h$ is a bounded weakly Hölder function. Notice that the added term $h$ not only generalizes the theory of $\mathbf{M y U 2}$ but it naturally emerges from the needs of multifractal analysis of Gibbs measures. The thermodynamic formalism presented in this paper is also based on good bounds for Perron-Frobenius operators which we obtain again by employing Nevanlinna theory after having made a suitable choice of a Riemannian metric on $\hat{\mathbb{C}}$. The emerging picture is nearly as complete as in the case of rational functions of Riemann sphere. We prove variational principle, the existence and uniqueness of Gibbs states (with the definition appropriately adapted for the transcendental case) and of equilibrium states of tame potentials, and we show that they coincide. There is also given a detailed description of spectral and asymptotic properties (spectral gap, Ionescu-Tulcea and Marinescu Inequality) of Perron-Frobenius operators, and their stochastic consequences such as the Central Limit Theorem, K-mixing, and exponential decay of correlations.

Thermodynamic formalism being interesting itself, we have also applied it to study the fractal structure of Julia sets. Already in MyU2 Bowen's formula was established identifying the Hausdorff dimension of the radial Julia set as the zero of the pressure function $t \mapsto \mathrm{P}\left(-t \log \left|f^{\prime}\right|_{\sigma}\right)$, and the real-analytic dependence of the Hausdorff dimension on a reference parameter was shown. Recall that the concept of the radial Julia set, i.e. points that do not escape to infinity was firmly introduced in UZ1 and an appropriate Bowen's formula for exponential functions $z \mapsto \lambda e^{z}$ was proved in [UZ2. There, also real-analytic dependence on $\lambda$ was proved. In the present paper we went further with applications of the developed thermodynamical formalism. Namely, we examined the finer fractal structure of the radial Julia sets by developing the multifractal analysis of Gibbs states of tame potentials. Here again, the theory turned out to be as complete as for hyperbolic rational functions. Indeed, the multifractal spectrum function is proved to be convex, real-analytic and to be the Legendre transform conjugate to the temperature function. Here, in the last chapter, we went even further, by showing that for a analytic family satisfying a two-sided version of the growth condition (1.1) in a uniform way, the multifractal spectrum function is real-analytic also with respect to the parameter. Such a fact, up to our knowledge, has not been so far proved even for hyperbolic rational functions nor even for the quadratic family $z \mapsto z^{2}+c$.

Looking for a moment at the content of our memoir, let us note that the Chapters 2, 3 , and and 4 deal with functions that satisfy various growth conditions, introductory treatment of the transfer operator along with the change of Riemannnian metric, application of Nevanlina's theory, most notably Borel sums, various concepts of Hölderness, and distortion properties. Chapters 5 and 6 cover the core part of the 
thermodynamic formalism, whereas Chapter 7 touches on more refined properties of Gibbs states and Perron-Frobenius operators. Here, the leading idea is to embed the potentials holomorphically into a complex-valued family of tame functions and to consider the corresponding Perron-Frobenius operators. These are demonstrated to depend holomorphically on the (complex) parameter. This technical fact along with the Kato-Rellich Perturbation Theorem for Linear Operators is a source of a number of interesting consequences. Among them real analyticity of topological pressure and other objects like eigenfunctions and contracting "remainders" produced in the process of developing the thermodynamic formalism. A uniform version of exponential decay of correlations finishes the Section 7.2.

Section 7.3, Derivatives of the Pressure Function, motivated by the appropriate parts of [PU] establishes formulas for the first and second derivatives of topological pressure. Even in the classical cases of distance expanding or subshift of finite type cases, this is not an easy task. In our present context, the calculations, especially of the second derivative, are tedious indeed. We have divided the proofs in several steps and provided a detailed ideas of each of them. One of the sources of technical difficulties is the fact that loosely tame potentials are unbounded and, therefore, do not belong to the Banach space of bounded Hölder continuous functions. This difficulty is taken care of by Lemma 7.9.

In Chapter 8, where the multifractal analysis is performed on the whole radial Julia set $J_{r}(f)$, we take fruits of all the previous sections, especially Section 6 . Real analyticity of the multifractal spectrum is established for all dynamically regular transcendental maps and Gibbs states of all tame potentials. The multifractal spectrum is also shown to be the Legendre conjugate of the temperature function. Volume Lemma, the Billingsley's type formula for the Hausdorff dimension of Gibbs measures of tame potentials, is proven and, as a by-product, Bowen's formula for the Hausdorff dimension of the radial Julia set $J_{r}(f)$ from $\mathbf{M y U 2}$ is reproved.

Fixing a family of transcendental functions that satisfy again certain natural uniform versions of condition (1.1) we perform the multifractal analysis for potentials of the form

$$
-t \log \left|f_{\lambda}^{\prime}\right|_{\sigma}+h,
$$

where $h$ is a real-valued bounded harmonic function defined on an open neighborhood of the Julia set of a fixed member of $\Lambda$. We show that the multifractal function $\mathcal{F}_{\phi}(\lambda, \alpha)$ depends real analytically not only on the multifractal parameter $\alpha$ but also on $\lambda$. As a by-product of our considerations in this chapter, we reproduce from MyU2], providing all details, the real-analytic dependence of $\operatorname{HD}\left(J_{r}\left(f_{\lambda}\right)\right)$ on $\lambda$ (Theorem 9.11). At the end of this chapter we provide a fairly easy sufficient condition for the multifractal spectrum not to degenerate. 


\section{CHAPTER 2}

\section{Balanced functions}

Here we introduce our class of functions. They are determined via growth conditions on the derivative that will be given in the next section. Such growth conditions are quite natural and very general in the context of meromorphic functions. We illustrate this with various examples in the remainder of this chapter.

In the definitions to follow appear some conditions on the growth of the derivative of the function. It is only necessary that they hold on the Julia set. Let us simply recall here that $\mathcal{F}_{f}$ designs the Fatou and $\hat{\mathcal{J}}(f)$ the Julia set of the function $f: \mathbb{C} \rightarrow \hat{\mathbb{C}}$. Since infinity is a point of indeterminacy for $f$ it is more convenient to work with the (finite) Julia set

$$
\mathcal{J}(f)=\hat{\mathcal{J}}(f) \cap \mathbb{C} .
$$

Precise definitions are given in Chapter 4

\subsection{Growth conditions}

We consider meromorphic functions $f: \mathbb{C} \rightarrow \hat{\mathbb{C}}$ of finite order $\rho=\rho(f)$ that satisfy the following conditions.

Definition 2.1 (Rapid derivative growth). A meromorphic function $f$ has rapid derivative growth if there are $\underline{\alpha}_{2}>\max \left\{0,-\alpha_{1}\right\}$ and $\kappa>0$ such that

$$
\left|f^{\prime}(z)\right| \geq \kappa^{-1}(1+|z|)^{\alpha_{1}}\left(1+|f(z)|^{\underline{\alpha}_{2}}\right)
$$

for all finite $z \in \mathcal{J}(f) \backslash f^{-1}(\infty)$.

Definition 2.2 (Balanced growth). The meromorphic function $f$ is balanced if there are $\kappa>0$, a bounded function $\left.\alpha_{2}: \mathcal{J}(f) \cap \mathbb{C} \rightarrow\left[\underline{\alpha}_{2}, \bar{\alpha}_{2}\right] \subset\right] 0, \infty[$ and $\alpha_{1}>-\underline{\alpha}_{2}=-\inf \alpha_{2}$ such that

$$
\kappa^{-1}(1+|z|)^{\alpha_{1}}\left(1+|f(z)|^{\alpha_{2}(z)}\right) \leq\left|f^{\prime}(z)\right| \leq \kappa(1+|z|)^{\alpha_{1}}\left(1+|f(z)|^{\alpha_{2}(z)}\right)
$$

for all finite $z \in \mathcal{J}(f) \backslash f^{-1}(\infty)$.

We will make some natural restrictions on the function $\alpha_{2}$ (given in Definition 2.5). Notice that most of our work does rely only on the weaker rapid growth condition. The balanced version of it is only used in the last two chapters.

Since we are interested in hyperbolic functions $f$ (the precise definition of hyperbolicity is also given in Chapter (4) we can and do assume that

$$
\left|f^{\prime}\right|_{\mid \mathcal{J}(f)} \geq c>0 \text { and } \quad|f|_{\mid \mathcal{J}(f)} \geq T>0 \text {. }
$$


The second condition means that $0 \in \mathcal{F}_{f}$. Under these assumptions the derivative growth condition (2.1) can then be reformulated in the following more convenient form:

There are $\underline{\alpha}_{2}>0, \alpha_{1}>-\underline{\alpha}_{2}$ and $\kappa>0$ such that

$$
\left|f^{\prime}(z)\right| \geq \kappa^{-1}|z|^{\alpha_{1}}|f(z)|^{\underline{\alpha}_{2}}
$$

for all $z \in \mathcal{J}(f) \backslash f^{-1}(\infty)$.

Similarly, the balanced condition (2.2) becomes

There are $\kappa>0$, a bounded function $\left.\alpha_{2}: \mathcal{J}(f) \rightarrow\left[\underline{\alpha}_{2}, \bar{\alpha}_{2}\right] \subset\right] 0, \infty[$ and a constant $\alpha_{1}>-\underline{\alpha}_{2}=-\inf \alpha_{2}$ such that

$$
\kappa^{-1}|z|^{\alpha_{1}}|f(z)|^{\alpha_{2}(z)} \leq\left|f^{\prime}(z)\right| \leq \kappa|z|^{\alpha_{1}}|f(z)|^{\alpha_{2}(z)}
$$

for all $z \in \mathcal{J}(f) \backslash f^{-1}(\infty)$,

Throughout the entire text we use the notations

$$
\alpha=\alpha_{1}+\underline{\alpha}_{2} \quad \text { and, for every } \tau \in \mathbb{R}, \quad \hat{\tau}=\alpha_{1}+\tau .
$$

Definition 2.3 (Dynamically regular functions). A balanced hyperbolic meromorphic function $f$ of finite order $\rho(f)$ is called dynamically regular. If $f$ satisfies only the rapid derivative growth condition then we call it dynamically semi-regular.

In the geometric applications of the thermodynamical formalism the following notion is useful.

Definition 2.4 (Divergence type). A meromorphic function $f$ is of divergence type if the series

$$
\Sigma(t, w)=\sum_{z \in f^{-1}(w)}|z|^{-t}
$$

diverges at the critical exponent (which is the order of the function $t=\rho ; w$ is any non Picard exceptional value). In the case $f$ is entire we assume instead of (2.6) that, for any $A, B>0$, there exists $R>1$ such that

$$
\int_{\log R}^{R} \frac{T(r)}{r^{\rho+1}} d r-B(\log R)^{1-\rho} \geq A
$$

where $T$ is the characteristic function of $f$.

In the entire case (2.6) is not sufficient for our needs. This is why we allow ourselves to modify in this case the usual notion of divergence type. This notion is in fact a condition on the growth of the characteristic function. For example, if

$$
\liminf _{r \rightarrow \infty} \frac{T(r)}{r^{\rho}}>0,
$$

then the function is of divergence type. 


\subsection{The precise form of $\alpha_{2}$}

The meaning of the exponent $\alpha_{1}$ and, in particular, of the $\alpha_{2}$-function deserves some clarifications and comments.

For entire functions the balanced growth condition (2.5) is in fact a condition on the logarithmic derivative of the function. Indeed, for all known balanced entire functions and, in particular, for the ones we describe below one has $\alpha_{2}=1$ and $\alpha_{1}=\rho-1$ with, as usual, $\rho$ being the order of the function. The balanced growth condition signifies then that the logarithmic derivative of the function is of polynomial growth of order $\rho-1$. For entire functions with bounded singular set this is a general fact (see Lemma 3.1 in MyU2 ).

For a meromorphic function $f$ with pole $b$ of multiplicity $q$, we have $\left|f^{\prime}\right| \asymp$ $|f|^{1+\frac{1}{q}}$ near the pole $b$. If $f$ satisfies the balanced growth condition then necessarily $\alpha_{2} \asymp 1+\frac{1}{q}$ near $b$. In order to be able to handle meromorphic functions with poles of different multiplicities we introduced the variable function $\alpha_{2}$. It must however satisfy the following condition.

Definition 2.5. If $f$ is entire then we suppose $\alpha_{2} \equiv 1$ 1]. If $f$ has poles then we suppose that

$$
\sup \left\{q_{b}, q_{b} \text { multiplicity of the pole } b\right\}<\infty
$$

and that

$$
\underline{\alpha}_{2}=\inf \left\{1+\frac{1}{q_{b}}, b \text { pole of } f\right\} \leq \alpha_{2} \leq \bar{\alpha}_{2}<\infty
$$

\subsection{Classical families}

We now present various classical families to which the theory of this memoir applies. First of all, the whole exponential family $f_{\lambda}(z)=\lambda \exp (z), \lambda \neq 0$, clearly satisfies the balanced growth condition with $\alpha_{1}=0$ and $\alpha_{2} \equiv 1$. More generally, if $P$ and $Q$ are arbitrary polynomials such that

$$
f(z)=P(z) \exp (Q(z))
$$

satisfies (2.3), then

$$
\left|f^{\prime}\right|=\frac{\left|P^{\prime}+Q^{\prime} P\right|}{|P|}|f| \asymp|z|^{\operatorname{deg}(Q)-1}|f|
$$

which explains that all these functions satisfy the balanced growth condition with $\alpha_{1}=\operatorname{deg}(Q)-1$ and $\alpha_{2} \equiv 1$. One can also consider functions

$$
f(z)=P \circ \exp (Q(z))
$$

where again $P, Q$ are polynomials such that (2.3) is satisfied. Then $f$ is again balanced with $\alpha_{1}=\operatorname{deg}(Q)-1$ and $\alpha_{2} \equiv 1$. Note that the order of these functions is $\rho=\operatorname{deg}(Q)$. Consequently $\frac{\rho}{\alpha}=1$.

Since one can replace in these considerations the exponential function by any arbitrary balanced meromorphic function $g$ one can produce in this way large families of balanced meromorphic functions. For example, if $P, Q$ are (non constant) polynomials such that $f=P \circ g \circ Q$ satisfies (2.3) then $f$ is balanced.

\footnotetext{
${ }^{1}$ In fact, only $\alpha_{2} \equiv c>0$ is needed.
} 
Assuming still (2.3), the following functions are also balanced:

The sine family. $f(z)=\sin (a z+b)$ where $a, b \in \mathbb{C}$ and $a \neq 0$.

The cosine-root family. $f(z)=\cos (\sqrt{a z+b})$ with again $a, b \in \mathbb{C}$ and $a \neq 0$. Note that here $\alpha_{1}=-\frac{1}{2}$ and $\alpha_{2} \equiv 1$ which explains that negative values of $\alpha_{1}$ should be considered in (2.4) and (2.5).

The tangent family. Certain solutions of Ricatti differential equations like, for example, the tangent family $f(z)=\lambda \tan (z), \lambda \neq 0$, and, more generally, the functions

$$
f(z)=\frac{A e^{2 z^{k}}+B}{C e^{2 z^{k}}+D} \quad \text { with } \quad A D-B C \neq 0 .
$$

The associated differential equations are of the form $w^{\prime}=k z^{k-1}\left(a+b w+c w^{2}\right)$ which explains that here $\alpha_{1}=k-1$ and $\alpha_{2} \equiv 2$.

Elliptic functions. All elliptic functions are balanced. Indeed, if $f: \mathbb{C} \rightarrow \hat{\mathbb{C}}$ is a doubly periodic meromorphic function, then there is $R>0$ such that every component $V_{b}$ of $f^{-1}(\{z \in \mathbb{C}:|z|>R\} \cup\{\infty\})$ is a bounded topological disc, and there is $\kappa>0$ such that for every pole $b$ and any $z \in V_{b} \backslash\{b\}$ we have

$$
\frac{1}{\kappa}|f(z)|^{1+\frac{1}{q_{b}}} \leq\left|f^{\prime}(z)\right| \leq \kappa|f(z)|^{1+\frac{1}{q_{b}}}
$$

where $q_{b}$ is the multiplicity of the pole $b$. From the periodicity of $f$ and the assumption $\left|f^{\prime}\right|_{\mid \mathcal{J}(f)} \geq c>0$ easily follows now that $f$ satisfies (2.5) with $\alpha_{1}=0$ and

$$
\underline{\alpha}_{2}=\inf \left\{1+\frac{1}{q_{b}}: b \in f^{-1}(\infty)\right\} .
$$

More generally, the preceding discussion shows that for any function $f$ that has at least one pole one always has

$$
\underline{\alpha}_{2} \leq \inf \left\{1+\frac{1}{q_{b}}: b \in f^{-1}(\infty)\right\} \text { and } \sup \left\{1+\frac{1}{q_{b}}: b \in f^{-1}(\infty)\right\} \leq \sup _{z \in \mathcal{J}(f)} \alpha_{2}(z) .
$$

\subsection{Functions with polynomial Schwarzian derivative}

The exponential and tangent functions are examples for which the Schwarzian derivative

$$
S(f)=\left(\frac{f^{\prime \prime}}{f^{\prime}}\right)^{\prime}-\frac{1}{2}\left(\frac{f^{\prime \prime}}{f^{\prime}}\right)^{2}
$$

is constant. By Möbius invariance of $S(f)$, functions like

$$
\frac{e^{z}}{\lambda e^{z}+e^{-z}} \text { and } \frac{\lambda e^{z}}{e^{z}-e^{-z}}
$$

also have constant Schwarzian derivative. Examples for which $S(f)$ is a polynomial are

and also

$$
f(z)=\int_{0}^{z} \exp (Q(\xi)) d \xi \quad, \quad Q \text { a polynomial, }
$$

$$
f(z)=\frac{a A i(z)+b B i(z)}{c A i(z)+d B i(z)} \quad \text { with } \quad a d-b c \neq 0
$$


and with $A i$ and $B i$ the Airy functions of the first and second kind. These a linear independent solutions of $g^{\prime \prime}-z g=0$ and, in general, if $g_{1}, g_{2}$ are linear independent solutions of

$$
g^{\prime \prime}+P g=0,
$$

then $f=\frac{g_{1}}{g_{2}}$ is a solution of the Schwarzian equation

$$
S(f)=2 P .
$$

Conversely, every solution of (2.10) can be written locally as a quotient of two linear independent solutions of the linear differential equation (2.9). Note that, if $g_{1}, g_{2}$ are two linear independent solutions of (2.9), then the Wronskian $W\left(g_{1}, g_{2}\right)$ has zero derivative and is therefore constant (and it is non-zero).

Nevanlinna Nev3 established that meromorphic functions with polynomial Schwarzian derivative are exactly the functions that have only finitely many asymptotical values and no critical values. Moreover, if such a function has a pole, then it is of order one. Consequently the maps of this class are locally injective. We also mention that any solution of (2.10) is of order $\rho=p / 2$, where $p=\operatorname{deg}(P)+2$, and it is of normal type of its order (cf. [H2]).

Theorem 2.6. Any meromorphic function $f$ with polynomial Schwarzian derivative is of divergence type and is balanced provided $\left|f^{\prime}\right|_{\mid \mathcal{J}(f)} \geq c>0$ with $\alpha_{1}=$ $\operatorname{deg}(S(f)) / 2$ and $\underline{\alpha}_{2} \in[1,2]$. Moreover, $\underline{\alpha}_{2} \equiv 2$ if all the asymptotical values of $f$ are finite.

Proof. The asymptotic properties of the solutions of (2.9) are well known due to work of Hille $([\mathbf{H 3}]$, see also $[\mathbf{H 2}]$. We follow $[\mathbf{L 1}])$. First of all, there are $p$ critical directions $\theta_{1}, \ldots, \theta_{p}$ which are given by

$$
\arg u+p \theta=0(\bmod 2 \pi)
$$

where $u$ is the leading coefficient of $P(z)=u z^{p-2}+\ldots$ In a sector

$$
S_{j}=\left\{\left|\arg z-\theta_{j}\right|<\frac{2 \pi}{p}-\delta ;|z|>R\right\},
$$

$R>0$ is sufficiently large and $\delta>0$, the equation (2.9) has two linear independent solutions

$$
\begin{aligned}
& g_{1}(z)=P(z)^{-\frac{1}{4}} \exp (i Z+o(1)) \quad \text { and } \\
& g_{2}(z)=P(z)^{-\frac{1}{4}} \exp (-i Z+o(1))
\end{aligned}
$$

where

$$
Z=\int_{2 R e^{i \theta_{j}}}^{z} P(t)^{\frac{1}{2}} d t=\frac{2}{p} u^{\frac{1}{2}} z^{\frac{p}{2}}(1+o(1)) \quad \text { for } \quad z \rightarrow \infty \text { in } S_{j} .
$$

Therefore, if $f$ is a meromorphic solution of the Schwarzian equation (2.10), then there are $a, b, c, d \in \mathbb{C}$ with $a d-b c \neq 0$ such that

$$
f(z)=\frac{a g_{1}(z)+b g_{2}(z)}{c g_{1}(z)+d g_{2}(z)} \quad, \quad z \in S_{j} .
$$

Observe that $f(z) \rightarrow a / c$ if $z \rightarrow \infty$ on any ray in $S_{j} \cap\left\{\arg z<\theta_{j}\right\}$ and that $f(z) \rightarrow b / d$ if $z \rightarrow \infty$ on any ray in $S_{j} \cap\left\{\arg z>\theta_{j}\right\}$. The asymptotic values of $f$ are given by all the $a / c, b / d$ corresponding to all the sectors $S_{j}, j=1, \ldots, p$. 
With this precise description of the asymptotic behavior of $f$ we can now proof Theorem 2.6 as follows. The Möbius transformation $\Phi(w)=\frac{a w+b}{c w+d}$ satisfies the differential equation

$$
w \Phi^{\prime}(w)=\alpha+\beta \Phi(w)+\gamma \Phi^{2}(w)
$$

where $\alpha=-a b / \delta, \beta=(a d+b c) / \delta, \gamma=-c d / \delta$ and $\delta=a d-b c$. If $g=\frac{g_{1}}{g_{2}}, g_{1}, g_{2}$ the functions given by (2.11), then the meromorphic function $f$ is $f=\Phi \circ g$ in the sector $S_{j}$ (see (2.12). Note that

$$
g^{\prime}=\frac{g_{1}^{\prime} g_{2}-g_{1} g_{2}^{\prime}}{g_{2}^{2}}=\frac{W\left(g_{1}, g_{2}\right)}{g_{2}^{2}}=\frac{k}{g_{2}^{2}}
$$

for some non-zero constant $k$. It follows then from (2.14) that

$$
f^{\prime}=\Phi^{\prime} \circ g g^{\prime}=\frac{1}{g}\left(\alpha+\beta f+\gamma f^{2}\right) \frac{k}{g_{2}^{2}}=\frac{k}{g_{1} g_{2}}\left(\alpha+\beta f+\gamma f^{2}\right) \text {. }
$$

Because of (2.11),

$$
g_{1}(z) g_{2}(z)=P(z)^{-\frac{1}{2}}(1+o(1)) \quad \text { for } z \rightarrow \infty \text { in } S_{j}
$$

This leads to

$$
\left|f^{\prime}(z)\right| \asymp|z|^{\frac{p}{2}-1}\left|\alpha+\beta f(z)+\gamma f(z)^{2}\right| \quad \text { for } z \in S_{j}
$$

Because of our standard assumption $\left|f^{\prime}\right|_{\mid \mathcal{J}(f)} \geq c>0$ it is clear now that $f$ is balanced in $\mathcal{J}(f) \cap S_{j}$ with $\alpha_{1}=\frac{p}{2}-1$ and $\alpha_{2} \equiv 1$ or $\alpha_{2} \equiv 2$ depending on $\gamma=-c d / \delta$. In fact, $\alpha_{2} \equiv 1$ precisely when $c d=0$. Notice that this implies that one of the asymptotic values is infinity.

The sectors $S_{j}, j=1, \ldots, p$, cover a neighborhood of infinity. Since $f$ can only have simple poles, $\left|f^{\prime}\right| \asymp|f|^{1+\frac{1}{q_{b}}}=|f|^{2}$ near a pole $b$. From a compactness argument follows now easily that $f$ is balanced, i.e. satisfies the condition (2.5), and that $\alpha_{2} \equiv 2$ in the case when all the asymptotic values of $f$ are finite.

It remains to check that $f$ is of divergence type. Take $z_{0} \in S_{j}$ and let $w_{0}=f\left(z_{0}\right)$ and $w=\Phi^{-1}\left(w_{0}\right)$. Now, $z \in f^{-1}\left(w_{0}\right) \cap S_{j}$ if and only if

$$
g(z)=\frac{g_{1}(z)}{g_{2}(z)}=\exp (2 i Z+o(1))=w
$$

(cf. 2.11). Recall that the order of $f$ is $\rho=p / 2$. From the 1 -periodicity of the exponential function and since $|Z| \asymp|z|^{p / 2}$ in $S_{j}$ it follows that

$$
\sum_{f(z)=w_{0}}|z|^{-\rho} \geq \sum_{\substack{g(z)=w \\ z \in S_{j}}}|z|^{-\frac{p}{2}}=\infty
$$

which precisely means that $f$ is of divergence type.

\subsection{Functions with rational Schwarzian derivative}

If $f$ is a meromorphic function with polynomial Schwarzian derivative and if $Q$ is any polynomial then it is easy to check that $g=f \circ Q$ is of divergence type and balanced with $\alpha_{1}=\operatorname{deg}(Q)-1+\operatorname{deg}(S(f)) / 2$ and $\alpha_{2}=\alpha_{2}(f)$ (still provided (2.3) holds). Since $g$ has critical points as soon as $\operatorname{deg}(Q)>1$ it cannot be a 
function with polynomial Schwarzian derivative. So here we have a first large class of balanced functions that are solutions of

$$
S(f)=R
$$

with $R$ a rational map. Functions with rational Schwarzian derivative have been studied by Elfving [Elf] who generalized the work of Nevanlinna cited above. These functions do also fit very well into our context. Let us simply focus on the following

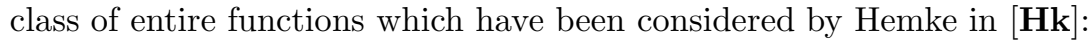

$$
f(z)=\int_{0}^{z} P(\xi) \exp (Q(\xi)) d \xi+c, \quad P, Q \text { polynomials }, c \in \mathbb{C} .
$$

These maps are precisely the entire functions with only finitely many singular values counted with multiplicity (see Corollary 2.13 of $[\mathbf{H k}]$ ).

Proposition 2.7. If $f$ is given by (2.15) such that $\left|f^{\prime}\right|_{\mid \mathcal{J}(f)} \geq c>0$, then $f$ is a balanced function with $\alpha_{1}=\operatorname{deg}(Q)-1$ and $\alpha_{2} \equiv 1$.

Proof. For $k=1, \ldots, \operatorname{deg}(Q)$ define

$$
\Phi_{k}=\frac{(2 k+1) \pi-\arg q}{\operatorname{deg}(Q)},
$$

where $q$ is the leading coefficient of $Q(z)=q z^{\operatorname{deg}(Q)}+\ldots$. Since $\exp \left(Q\left(R \varepsilon^{i \Phi_{k}}\right)\right)$ decreases very fast when $R \rightarrow \infty, s_{k}=\lim _{R \rightarrow \infty} f\left(R \varepsilon^{i \Phi_{k}}\right)$ is a finite asymptotical value of $f$. For $z \in \mathbb{C}$ choose $k$ such that

$$
\Phi_{k}-\frac{\pi}{\operatorname{deg}(Q)} \leq \arg z<\Phi_{k}+\frac{\pi}{\operatorname{deg}(Q)}
$$

and define $\bar{s}(z)=s_{k}$. Lemma $4.1 \mathrm{in}[\mathbf{H k}]$ states that

$$
f(z)=\bar{s}(z)+\frac{P(z) e^{Q(z)}}{Q^{\prime}(z)}+\mathcal{O}\left(|z|^{\operatorname{deg}(P)-\operatorname{deg}(Q)}\right) e^{Q(z)} \quad \text { for }|z| \geq R>0 .
$$

It follows that

$$
|f(z)-\bar{s}(z)|\left|Q^{\prime}(z)\right|=\left|f^{\prime}(z)\right|\left|1+\mathcal{O}\left(|z|^{\operatorname{deg}\left(Q^{\prime}\right)-\operatorname{deg}(Q)}\right)\right|
$$

which implies

$$
\left|f^{\prime}(z)\right| \asymp\left|Q^{\prime}(z)\right||f(z)-\bar{s}(z)| \text { for }|z| \geq R
$$

and the assertion follows.

\subsection{Uniform balanced growth}

In Chapter 8 we deal with analytic families of dynamically regular meromorphic functions. More precisely, the Speiser class $\mathcal{S}$ is the set of meromorphic functions $f: \mathbb{C} \rightarrow \hat{\mathbb{C}}$ that have a finite set of singular values $\operatorname{sing}\left(f^{-1}\right)$. We will work in the subclass $\mathcal{S}_{0}$ which consists in the functions $f \in \mathcal{S}$ that have a strictly positive and finite order $\rho=\rho(f)$ and that are of divergence type. Fix $\Lambda$, an open subset of $\mathbb{C}^{N}$, $N \geq 1$. Let

$$
\mathcal{M}_{\Lambda}=\left\{f_{\lambda}\right\}_{\lambda \in \Lambda} \subset \mathcal{S}_{0}
$$

be a holomorphic family of dynamically regular meromorphic functions such that the singular points $\operatorname{sing}\left(f_{\lambda}^{-1}\right)=\left\{a_{1, \lambda}, \ldots, a_{d, \lambda}\right)$ depend continuously on $\lambda \in \Lambda$. 
Definition 2.8 (Bounded deformation). A family $\mathcal{M}_{\Lambda}$ is of bounded deformation if there is $M>0$ such that for all $j=1, \ldots, N$

$$
\left|\frac{\partial f_{\lambda}(z)}{\partial \lambda_{j}}\right| \leq M\left|f_{\lambda}^{\prime}(z)\right|, \quad \lambda \in \Lambda \text { and } z \in \mathcal{J}\left(f_{\lambda}\right) \text {. }
$$

We will see that this bounded deformation condition yields the existence of a holomorphic motion

$$
z \in \mathcal{J}\left(f_{\lambda^{0}}\right) \mapsto z_{\lambda}=G_{\lambda}(z) \in \mathcal{J}\left(f_{\lambda}\right)
$$

that conjugates the dynamics and has the additional property that $G_{\lambda}$ converges to the identity uniformly on the whole plane as $\lambda \rightarrow \lambda^{0}$.

Definition 2.9 (Uniformly balanced). A family $\mathcal{M}_{\Lambda}$ is uniformly balanced provided every $f \in \mathcal{M}_{\Lambda}$ satisfies the condition (2.2) with $\kappa, \alpha_{1}, \alpha_{2}$ independent of $f \in \mathcal{M}$. Concerning $\alpha_{2}$, this means that for every $z \in \mathcal{J}\left(f_{\lambda^{0}}\right)$ the map

$$
\lambda \in \Lambda \mapsto \alpha_{2, \lambda}\left(z_{\lambda}\right)
$$

is constant.

Uniform balanced growth holds for various families of meromorphic functions. Here are some examples.

Proposition 2.10. Let $f: \mathbb{C} \rightarrow \hat{\mathbb{C}}$ be either the sine, tangent, exponential or the Weierstrass elliptic function and let $f_{\lambda}(z)=f\left(\lambda_{d} z^{d}+\lambda_{d-1} z^{d-1}+\ldots+\lambda_{0}\right), \lambda=$ $\left(\lambda_{d}, \lambda_{d-1}, \ldots, \lambda_{0}\right) \in \mathbb{C}^{*} \times \mathbb{C}^{d}$. Suppose $\lambda^{0}$ is a parameter such that $f_{\lambda^{0}}$ is topologically hyperbolic. Then there is a neighbourhood $U$ of $\lambda^{0}$ such that $\mathcal{M}_{U}=\left\{f_{\lambda} ; \lambda \in U\right\}$ is of uniform balanced growth.

Remark 2.11. Instead of the Weierstrass elliptic function one can take here any other elliptic function. This follows immediately from the above discussion on elliptic functions. Note that then $\alpha_{2}$ cannot be taken constant since the poles of such functions can have different multiplicities.

Proof. All the functions $f$ mentioned have only finitely many singular values, they are in the Speiser class. The function $f_{\lambda^{0}}$ being in addition topologically hyperbolic, its singular values are attracted by attracting cycles. As we already remarked in the previous section, this is a stable property in the sense that there is a neighbourhood $U$ of $\lambda^{0}$ such that all the functions of $\mathcal{M}_{U}=\left\{f_{\lambda} ; \lambda \in U\right\}$ have the same property. In particular, no critical point of $f_{\lambda}$ is in $J\left(f_{\lambda}\right)$. The function $f$ satisfies a differential equation of the form

$$
\left(f^{\prime}\right)^{p}=Q \circ f
$$

with $Q$ a polynomial whose zeros are contained in $\sin g\left(f^{-1}\right)$. For example, in the case when $f$ is the Weierstrass elliptic function then

$$
\left(f^{\prime}\right)^{2}=4\left(f-e_{1}\right)\left(f-e_{2}\right)\left(f-e_{3}\right)
$$

with $e_{1}, e_{2}, e_{3}$ the critical values of $f$. Let $\lambda \in U$ and denote $P_{\lambda}(z)=\lambda_{d} z^{d}+$ $\lambda_{d-1} z^{d-1}+\ldots+\lambda_{0}$. Since

$$
\left(f_{\lambda}^{\prime}\right)^{p}=\left(f^{\prime} \circ P_{\lambda} P_{\lambda}^{\prime}\right)^{p}=Q \circ f_{\lambda}\left(P_{\lambda}^{\prime}\right)^{p}
$$


and $f_{\lambda}(z) \neq 0$ for all $z \in J\left(f_{\lambda}\right)$, the polynomials $P_{\lambda}^{\prime}$ and $Q$ do not have any zero in $J\left(f_{\lambda}\right)$. Consequently

$$
\left|P_{\lambda}^{\prime}(z)\right| \asymp|z|^{d-1} \quad \text { and } \quad|Q(z)| \asymp|z|^{q} \quad \text { on } \quad J\left(f_{\lambda}\right)
$$

with $q=\operatorname{deg}(Q)$. Moreover, restricting $U$ if necessary, the involved constants can be chosen to be independent of $\lambda \in U$. Therefore,

$$
\left|f_{\lambda}^{\prime}(z)\right| \asymp\left|f_{\lambda}(z)\right|^{\frac{q}{p}}|z|^{d-1}
$$

for $z \in J\left(f_{\lambda}\right)$ and $\lambda \in U$. We verified the uniform balanced growth condition with $\alpha_{1}=d-1$ and $\alpha_{2}=\frac{q}{p}$ depending on the choice of $f$. In the case of the Weierstrass elliptic function one has $\alpha_{2}=3 / 2$. 



\section{CHAPTER 3}

\section{Transfer operator and Nevanlinna Theory}

\subsection{Choice of a Riemannian metric and transfer operator}

It was observed in MyU2 that one can build the thermodynamical formalism of the very general class of dynamically semi-regular meromorphic functions provided that one works with the right Riemannian metric space $(\mathbb{C}, d \sigma=\gamma|d z|)$. More precisely, if $\phi=-t \log \left|f^{\prime}\right|_{\sigma}$ is a geometric potential with $t>\rho / \alpha$ and with

$$
\left|f^{\prime}(z)\right|_{\sigma}=\frac{d \sigma(f(z))}{d \sigma(z)}=\left|f^{\prime}(z)\right| \frac{\gamma(f(z))}{\gamma(z)}
$$

the derivative of $f$ with respect to the metric $\sigma$, then the right choice of the metric is

$$
d \sigma(z)=d \sigma_{\tau}(z)=\frac{|d z|}{1+|z|^{\tau}}
$$

where $\tau \in\left(0, \underline{\alpha}_{2}\right)$ is such that $t>\rho / \hat{\tau}>\rho / \alpha$ (for simplicity we will denote the metric $\sigma_{\tau}$ just by $\tau$ ). Since we only work on the Julia set $\mathcal{J}(f)$ which we supposed to be at some distance from the origin (see (2.3) ) we can and do work with the simpler form of the metric

$$
d \tau(z)=|z|^{-\tau}|d z| .
$$

The main idea in MyU2 was that one could show, with the help of Nevanlinna theory, that the (geometric) transfer operator

$$
\mathcal{L}_{t} \varphi(w)=\sum_{z \in f^{-1}(w)}\left|f^{\prime}(z)\right|_{\tau}^{-t} \varphi(z) \quad, \quad \varphi \in C_{b}(\mathcal{J}(f)),
$$

is bounded for all $t>\rho / \hat{\tau}$. In Chapter 5 we systematical deal with a more general class of potentials. But let us explain at the moment, with the example of the above potentials $-t \log \left|f^{\prime}(z)\right|_{\tau}$, why Nevanlinna Theory plays the first rate role in the understanding of the transfer operator. Suppose $f$ is a function that satisfies the balanced growth condition (2.5). Then we have (still under the assumption (2.3)

$$
|z|^{\hat{\tau}} \preceq|z|^{\hat{\tau}}|f(z)|^{\alpha_{2}-\tau} \preceq\left|f^{\prime}(z)\right|_{\tau} \preceq|z|^{\hat{\tau}}|f(z)|^{\bar{\alpha}_{2}-\tau}, \quad z \in \mathcal{J}(f) \backslash f^{-1}(\infty) .
$$

Here and in the whole text the symbols $\asymp$ and $\preceq$ signify that equality respectively inequality holds up to a multiplicative constant that is independent of the involved variables. Notice that the left hand inequality of (3.4) is still valid under the weaker growth condition (2.4). Therefore we have for a dynamically semi-regular function $f$ the estimation

$$
\mathcal{L}_{t} \mathbb{1}(w) \preceq \frac{1}{|w|^{\alpha_{2}}-\tau} \sum_{z \in f^{-1}(w)}|z|^{-\hat{\tau} t} \preceq \sum_{z \in f^{-1}(w)}|z|^{-\hat{\tau} t} \quad, w \in \mathcal{J}(f),
$$


and this last sum, which we also call Borel sum, is very well known in Nevanlinna theory. The next Section provides all necessary details related to the behavior of this sum which, in particular, give boundedness of the transfer operator.

\subsection{Nevanlinna Theory and Borel Sums}

The reader may consult, for example, Hy, [H1, JV], [Nev1, Nev2 or CY] for a detailed exposition on meromorphic functions and on Nevanlinna theory. In the whole text we use the terminology meromorphic function for a transcendental meromorphic function $f$ of the plane $\mathbb{C}$ into the sphere $\hat{\mathbb{C}}$ and we always suppose that $f$ is of finite order

$$
\rho=\rho(f)=\limsup _{r \rightarrow \infty} \frac{\log T(r)}{\log r}<\infty .
$$

Here and in the following we use the standard notation of Nevanlinna theory. For example, $n(r, a)$ is the number of $a$-points of modulus at most $r, N(r, a)$ is defined by $d N(r, a)=n(r, a) / r$ and $T(r)$ is the characteristic of $f$ (more precisely the Ahlfors-Shimizu version of it; these two different definitions of the characteristic function only differ by a bounded amount). Notice that $f$ is of finite order $\rho$ if and only if the integral

$$
\int^{\infty} \frac{T(r)}{r^{u+1}} d r
$$

converges for $u>\rho$ and diverges if $u<\rho$. This integral may converge or diverge for the critical exponent $u=\rho$. Following Valiron we introduce the following (and remember that for entire functions we take the different version given in (2.7).

Definition 3.1. A meromorphic function $f$ of finite order $\rho$ is of divergence type if

$$
\int^{\infty} \frac{T(r)}{r^{\rho+1}} d r=\infty
$$

More adapted for our concerns will be the characterization of the order and the divergence type in terms of the sum

$$
\Sigma(u, a)=\sum_{\substack{f(z)=a \\ z \neq 0}}|z|^{-u}, \quad a \in \hat{\mathbb{C}} .
$$

The relation between this sum and the integral (3.5) goes via the average counting number $N(r, a)$ and Nevanlinna's main theorems. The first main theorem (FMT) as stated in [Er] or in [H1, p. 216] yields

Corollary 3.2 (of FMT). For every $a \in \hat{\mathbb{C}}$ there is $\Theta_{a}>0$ such that

$$
N(r, a)-\Theta_{a} \leq T(r) \quad \text { for all } r>0 .
$$

In the case $f(0) \neq a$ one has $\Theta_{a}=-\log [f(0), a]$ where $[a, b]$ denotes the chordal distance on the Riemann sphere (with in particular $[a, b] \leq 1$ for all $a, b \in \hat{\mathbb{C}}$ ). 
From the second main theorem (SMT) of Nevanlinna we need the following version which is from [Nev1, p. 257] ([Nev2 , p. 255] or again [H1]) and which is valid only since $f$ is supposed to be of finite order.

Corollary 3.3 (of SMT). Let $a_{1}, a_{2}, a_{3} \in \hat{\mathbb{C}}$ be distinct points. Then

$$
T(r) \leq N\left(r, a_{1}\right)+N\left(r, a_{2}\right)+N\left(r, a_{3}\right)+S(r)
$$

for every $r>0$ and with $S(r)=\mathcal{O}(\log (r))$.

Putting together these two results one has for any $r>0$, any three distinct points $a_{1}, a_{2}, a_{3} \in \hat{\mathbb{C}}$ and any $a \in \hat{\mathbb{C}}$ that

$$
N(r, a)-\Theta_{a} \leq T(r) \leq N\left(r, a_{1}\right)+N\left(r, a_{2}\right)+N\left(r, a_{3}\right)+S(r) .
$$

The error term $S(r)$ also depends on the points $a_{j}$. It has been studied in detail and sharp estimates are known. The following results from Hinkkanen's paper [Hk] and also from Cherry-Ye's book [CY]. We use here the notion of hyperbolicity which is defined in the next section.

Lemma 3.4. Let $f$ be a hyperbolic meromorphic function of finite order $\rho$ that is normalized such that $0 \in D(0, T) \subset \mathcal{F}_{f}, f(0) \notin\{0, \infty\}$ and $f^{\prime}(0) \neq 0$. Then, for every $\Delta<T / 4$, there exists $C_{1}=C_{1}(\Delta)>0$ and $C_{2}>0$ such that

$$
4 N(R+\Delta, a) \geq T(R)-(3 \rho+1) \log R-C_{1}-C_{2} \log |a|
$$

for every $a \in \mathcal{J}(f)$ and every $R>T$.

Proof. Since $f$ is expanding there is $c>0$ such that $\left|f^{\prime}(z)\right| \geq c>0$ for all $z \in \mathcal{J}(f)$. Let $0<\Delta^{\prime}<\min \{\delta(f), T\}$ such that $\Delta=2 K \Delta^{\prime} / c<T / 4$ where $K$ is an appropriate Koebe distortion constant. Consider then $a \in \mathcal{J}(f)$ and $a^{\prime} \in D\left(a, \Delta^{\prime}\right)$. Since all the inverse branches of $f$ are well defined on $D\left(a, 2 \Delta^{\prime}\right)$ we have

$$
n(r+\Delta, a) \geq n\left(r, a^{\prime}\right) \quad, \quad r>0 .
$$

Consequently

$$
\begin{aligned}
N\left(R, a^{\prime}\right) & =\int_{0}^{R} \frac{n\left(r, a^{\prime}\right)}{r} d r \leq \int_{0}^{R} \frac{n(r+\Delta, a)}{r} d r \\
& =\int_{\Delta}^{R+\Delta} \frac{n(t, a)}{t} \frac{t}{t-\Delta} d t \leq \frac{T}{T-\Delta} \int_{T}^{R+\Delta} \frac{n(t, a)}{t} d t \\
& \leq \frac{4}{3} N(R+\Delta, a) \text { for every } R>T .
\end{aligned}
$$

Choose now $a_{1}, a_{2}, a_{3} \in D\left(a, \Delta^{\prime}\right)$, any three points that satisfy $\left|a_{i}-a_{j}\right| \geq \Delta^{\prime} / 3$ for all $i \neq j$. It follows then from the sharp form of SMT given in $\underline{\mathbf{H k}}$, the fact that $f$ is of finite order, along with the normalisations stated in the lemma that

$$
\begin{aligned}
4 N(R+\Delta, a) & \geq \sum_{i=1}^{3} N\left(R, a_{i}\right) \geq T(R)-S\left(R, a_{1}, a_{2}, a_{3}\right) \\
& \geq T(R)-(3 \rho+1) \log R-C_{1}(\Delta)-C_{2} \log |a|
\end{aligned}
$$

for every $a \in \mathcal{J}(f)$ and for all $R>T$. 
It follows from SMT that the convergence of the integral (3.5) implies the convergence of

$$
\int^{\infty} \frac{N(r, a)}{r^{u+1}} d r
$$

for all $a \in \hat{\mathbb{C}}$. Conversely, if the integral (3.5) diverges then (3.8) also diverges for all but at most two (the Picard exceptional values) points $a \in \hat{\mathbb{C}}$.

Let us now come back to the sum (3.6). If $0<r_{0}<r$, then by the definition of the Riemann-Stieltjes integral and with integration by parts,

$$
\begin{aligned}
& \sum_{\substack{f(z)=a \\
r_{0}<|z|<r}}|z|^{-u}=\int_{r_{0}}^{r} \frac{d n(t, a)}{t^{u}}=\frac{n(r, a)}{r^{u}}-\frac{n\left(r_{0}, a\right)}{r_{0}^{u}}+u \int_{r_{0}}^{r} \frac{n(t, a) d t}{t^{u+1}} \\
& \quad=\frac{n(r, a)}{r^{u}}-\frac{n\left(r_{0}, a\right)}{r_{0}^{u}}+u\left(\frac{N(r, a)}{r^{u}}-\frac{N\left(r_{0}, a\right)}{r_{0}^{u}}\right)+u^{2} \int_{r_{0}}^{r} \frac{N(t, a) d t}{t^{u+1}} .
\end{aligned}
$$

It follows now easily that the convergence behavior of $\Sigma(u, a)$ is the same as the one of the integral (3.8). We thus have

Theorem 3.5 (Borel-Picard). Let $f$ be a meromorphic function and let $\mathcal{E}_{f} \subset \hat{\mathbb{C}}$ be the set of the (at most two) Picard exceptional values. Then $f$ is of finite order $\rho$ if and only if

$$
\begin{aligned}
& \Sigma(u, a)<\infty \text { if } u>\rho \text { and } \\
& \Sigma(u, a)=\infty \text { if } u<\rho
\end{aligned}
$$

for all $a \in \hat{\mathbb{C}} \backslash \mathcal{E}_{f}$. Moreover, $\Sigma(\rho, a)=\infty$ for some $a \in \hat{\mathbb{C}}$ if and only if

$$
\Sigma(\rho, a)=\infty \text { for all } a \in \hat{\mathbb{C}} \backslash \mathcal{E}_{f} .
$$

The following uniform estimate is crucial for our needs.

Proposition 3.6. Let $f$ be meromorphic of finite order $\rho$ and let $\mathcal{K} \subset \hat{\mathbb{C}}$ such that $f(0) \notin \overline{\mathcal{K})>0}$. Then, for every $u>\rho$, there is $M_{u}>0$ such that

$$
\Sigma(u, a)=\sum_{f(z)=a} \frac{1}{|z|^{u}} \leq M_{u} \quad \text { for all } a \in \mathcal{K} .
$$

Proof. Let $0<r_{0}<\operatorname{dist}\left(0, f^{-1}(\mathcal{K})\right)$. Then $n\left(r_{0}, a\right)=N\left(r_{0}, a\right)=0$ and

$$
\sum_{\substack{f(z)=a \\ r_{0}<|z|<r}}|z|^{-u}=\frac{n(r, a)}{r^{u}}+u \frac{N(r, a)}{r^{u}}+u^{2} \int_{r_{0}}^{r} \frac{N(t, a) d t}{t^{u+1}}
$$

for every $a \in \mathcal{K}$ and $r>r_{0}$. We have $\lim _{r \rightarrow \infty} \frac{n(r, a)}{r^{u}}=\lim _{r \rightarrow \infty} \frac{N(r, a)}{r^{u}}=0$ since $u>\rho$. It follows from the assumption $\operatorname{dist}\left(0, f^{-1}(\mathcal{K})\right)>0$ that the constant $\Theta_{a}$ 
in FMT (Corollary 3.2) can be chosen to be independent of $a \in \mathcal{K}$. It follows that there is $A_{u}>0$ such that

$$
\Sigma(u, a) \leq \int_{r_{0}}^{\infty} \frac{T(r)}{r^{u+1}} d r+A_{u}=: M_{u}
$$

for every $a \in \mathcal{K}$. 



\section{CHAPTER 4}

\section{Preliminaries, Hyperbolicity and Distortion Properties.}

\subsection{Dynamical preliminaries and hyperbolicity}

For a general introduction of the dynamical aspects of meromorphic functions we refer to the survey article of Bergweiler [Bw1. We collect here the properties of interest for our concerns. The Fatou set of a meromorphic function $f: \mathbb{C} \rightarrow \hat{\mathbb{C}}$ is denoted by $\mathcal{F}_{f}$. It is defined as usual as to be the set of points $z \in \mathbb{C}$ for which there exists a neighborhood $U$ of $z$ on which all the iterates $f^{k}, k \geq 1$, of the function $f$ are defined and are normal. The complement is the Julia set $\hat{\mathcal{J}}(f)=\hat{\mathbb{C}} \backslash \mathcal{F}_{f}$. We write

$$
\mathcal{J}(f)=\hat{\mathcal{J}}(f) \cap \mathbb{C} .
$$

By Picard's theorem, there are at most two points $z_{0} \in \hat{\mathbb{C}}$ that have finite backward orbit $\mathcal{O}^{-}\left(z_{0}\right)=\bigcup_{n \geq 0} f^{-n}\left(z_{0}\right)$. The set of these points is the exceptional set $\mathcal{E}_{f}$. In contrast to the situation of rational maps it may happen that $\mathcal{E}_{f} \subset \hat{\mathcal{J}}(f)$. Iversen's theorem [Iv, $\mathbf{N e v 1}$ asserts that every $z_{0} \in \mathcal{E}_{f}$ is an asymptotic value. Consequently, $\mathcal{E}_{f} \subset \operatorname{sing}\left(f^{-1}\right)$ the set of critical and finite asymptotic values. The post-critical set $\mathcal{P}_{f}$ is defined to be the closure in the plane of

$$
\bigcup_{n \geq 0} f^{n}\left(\operatorname{sing}\left(f^{-1}\right) \backslash f^{-(n-1)}(\infty)\right) .
$$

The Julia set splits into two dynamically different subsets. First, there is the escaping set

$$
I_{\infty}(f)=\left\{z \in \mathcal{J}(f) ; \lim _{n \rightarrow \infty} f^{n}(z)=\infty\right\} .
$$

And, more importantly to us, its complement, the radial (or conical) Julia set

$$
\mathcal{J}_{r}(f)=\mathcal{J}(f) \backslash I_{\infty}(f) .
$$

The Hausdorff dimension of the radial Julia set will be called hyperbolic dimension of the function $f$.

Remark 4.1. We like to mention that L. Rempe in Rem introduced a different radial Julia set. Let us denote it by $\Lambda_{f}$. A point $z \in \mathcal{J}(f)$ is a radial point of $\Lambda_{f}$ if there is $\delta>0$ and $n_{j} \rightarrow \infty$ such that

$$
f^{n_{j}}: \operatorname{Comp}_{z}\left(D_{\sigma}\left(f^{n_{j}}(z), \delta\right)\right) \longrightarrow D_{\sigma}\left(f^{n_{j}}(z), \delta\right)
$$

is univalent where, this time, $\sigma$ stands for the spherical metric in $\hat{\mathbb{C}}$. In other words, there are arbitrary small neighborhoods of $z$ that can be zoomed in a univalent way by some iterate of the function $f$ to a disk of a fixed spherical size. This definition naturally emerges from the notion of conical limit set of Kleinian groups or the conical Julia set from rational functions. 
For our needs it is more convenient to use $\mathcal{J}_{r}(f)$ and both sets are closely related for hyperbolic functions. Indeed, for a hyperbolic function $f$ we always have $\mathcal{J}_{r}(f) \subset \Lambda_{f}$ and we will see that the difference $\Lambda_{f} \backslash \mathcal{J}_{r}(f)$ is dynamically insignificant in the sense that, firstly, all the Gibbs measures and equilibrium states have total mass on $\mathcal{J}_{r}(f)$ (Proposition 5.21) and, secondly, both sets have same Hausdorff dimension (Proposition 8.4). In particular, in the above definition of the hyperbolic dimension one can take either one of these radial sets. Notice also that L. Rempe showed that the hyperbolic dimension equals the supremum of the hyperbolic subsets of $\mathcal{J}(f)$ (which is indeed the usual definition for the hyperbolic dimension).

A classical fact based on Montel's theorem and the density of repelling cycles is that if $U$ is any open set with nonempty intersection with the Julia set and if $K$ is any compact subset of $\mathcal{J}(f)$, then there is $N \geq 0$ such that $f^{N}(U) \supset K$. The following is more convenient for our needs.

Lemma 4.2. Let $\delta>0$ and denote $U_{w}=D(w, \delta)$. For any $R>0$ there exists $N=N(R) \geq 0$ such that, if $K=\mathcal{J}(f) \cap \bar{D}(0, R)$, then $f^{N}\left(U_{w}\right) \supset K$ for any $w \in K$

Proof. Suppose to the contrary that there exists $R>0$ and, for any $N \geq 0$, $w_{N} \in K=\mathcal{J}(f) \cap \bar{D}(0, R)$ with $K \backslash f^{N}\left(U_{w_{N}}\right) \neq \emptyset$. We may suppose that $w_{N} \rightarrow$ $w \in K$. But then there is $N_{0} \geq 0$ such that $f^{N}(D(w, \delta / 2))$ does not contain $K$ for any $N \geq N_{0}$. This is impossible.

4.1.1. Hyperbolicity. Let us introduce the following definitions.

Definition 4.3. A meromorphic function $f$ is called topologically hyperbolic if

$$
\delta(f):=\frac{1}{4} \operatorname{dist}\left(\mathcal{J}(f), \mathcal{P}_{f}\right)>0 .
$$

and it is called expanding if there is $c>0$ and $\gamma>1$ such that

$$
\left|\left(f^{n}\right)^{\prime}(z)\right| \geq c \gamma^{n} \quad \text { for all } z \in \mathcal{J}(f) \backslash f^{-1}(\infty) .
$$

A topologically hyperbolic and expanding function is called hyperbolic.

The Julia set of a hyperbolic function is never the whole sphere. We thus may and we do assume that the origin $0 \in \mathcal{F}_{f}$ is in the Fatou set (otherwise it suffices to conjugate the map by a translation). This means that there exists $T>0$ such that

$$
D(0, T) \cap \mathcal{J}(f)=\emptyset .
$$

Here we simply justified and quantified the second part of the assumption (2.3).

It is well known that in the context of rational functions topological hyperbolicity and expanding property are equivalent. Neither implication is established for transcendental functions. However, under the rapid derivative growth condition (2.4) with $\alpha_{1} \geq 0$ topological hyperbolicity implies hyperbolicity.

Proposition 4.4. Every topologically hyperbolic meromorphic function satisfying the rapid derivative growth condition with $\alpha_{1} \geq 0$ is expanding, and consequently, hyperbolic. 
Proof. Let us fix $\gamma \geq 2$ such that $\gamma \kappa^{-1} T^{\alpha} \geq 2$. In view of rapid derivative growth (2.4) and (4.1)

$$
\left|f^{\prime}(z)\right| \geq \kappa^{-1} T^{\alpha} \quad \text { for all } z \in \mathcal{J}(f)
$$

and

$$
\left|f^{\prime}(z)\right| \geq \gamma \quad \text { for all } z \in f^{-1}(\mathcal{J}(f) \backslash D(0, R))
$$

provided $R>0$ has been chosen sufficiently large. In addition we need the following.

Claim: There exists $p \geq 1$ such that

$$
\left|\left(f^{n}\right)^{\prime}(z)\right| \geq \gamma \text { for all } n \geq p \text { and } z \in \bar{D}(0, R) \cap \mathcal{J}(f) .
$$

Indeed, suppose on the contrary that for some $n_{p} \rightarrow \infty$ and $z_{p} \in \bar{D}(0, R) \cap \mathcal{J}(f)$ we have

$$
\left|\left(f^{n_{p}}\right)^{\prime}\left(z_{p}\right)\right|<\gamma
$$

Put $\delta=\delta(f)$. Then for every $p \geq 1$ there exists a unique holomorphic branch $f_{*}^{-n_{p}}: D\left(f^{n_{p}}\left(z_{p}\right), 2 \delta\right) \rightarrow \mathbb{C}$ of $f^{-n_{p}}$ sending $f^{n_{p}}\left(z_{p}\right)$ to $z_{p}$. It follows from $\frac{1}{4}$ Koebe's Distortion Theorem (cf. Lemma 4.6) and (4.4) that

$$
f_{*}^{-n_{p}}\left(D\left(f^{n_{p}}\left(z_{p}\right), 2 \delta\right)\right) \supset D\left(z_{p}, \delta /(2 \gamma)\right)
$$

or, equivalently, that $f^{n_{p}}\left(D\left(z_{p}, \delta /(2 \gamma)\right)\right) \subset D\left(f^{n_{p}}\left(z_{p}\right), 2 \delta\right)$. Passing to a subsequence we may assume without loss of generality that the sequence $\left\{z_{p}\right\}_{p=1}^{\infty}$ converges to a point $z \in \bar{D}(0, R) \cap \mathcal{J}(f)$. Since $D\left(\mathcal{P}_{f}, 2 \delta\right) \cap D\left(f^{n_{p}}\left(z_{p}\right), 2 \delta\right)=\emptyset$ for every $p \geq 1$, it follows from Montel's theorem that the family $\left\{\left.f^{n_{p}}\right|_{D\left(z,(2 \gamma)^{-1} \delta\right)}\right\}_{p=1}^{\infty}$ is normal, contrary to the fact that $z \in \mathcal{J}(f)$. The claim is proved.

Let $p=p(\gamma, R) \geq 1$ be the number produced by the claim. It remains to show that

$$
\left|\left(f^{2 p}\right)^{\prime}(z)\right| \geq 2>1 \quad \text { for every } z \in \mathcal{J}(f) .
$$

This formula holds if $\left|f^{j}(z)\right|>R$ for $j=0,1, \ldots, p$ because of (4.2), (4.3) and the choice of $\gamma$. If $\left|f^{j}(z)\right| \leq R$ for some $0 \leq j \leq p$, the conclusion follows from (4.2) and the claim.

4.1.2. Analytic families. Let us recall that the class of Speiser $\mathcal{S}$ consists in the functions $f$ that have a finite set of singular values $\operatorname{sing}\left(f^{-1}\right)$. The classification of the periodic Fatou components is the same as the one of rational functions because any map of $\mathcal{S}$ has no wandering nor Baker domains [Bw1. Consequently, if $f \in \mathcal{S}$ then $f$ is topologically hyperbolic if and only if the orbit of every singular value converges to one of the finitely many attracting cycles of $f$. This last property is stable under perturbation, a fact that is needed for the next remark:

Fact 4.5. Let $f_{\lambda^{0}} \in \mathcal{H}$ be a hyperbolic function and $U \subset \Lambda$ an open neighborhood of $\lambda^{0}$ such that, for every $\lambda \in U, f_{\lambda}$ satisfies the balanced growth condition (2.5) with $\kappa>0, \alpha_{1} \geq 0$ and $\underline{\alpha}_{2}>0$ independent of $\lambda \in U$. Then, replacing $U$ by some smaller neighborhood if necessary, all the $f_{\lambda}$ satisfy the expanding property for some $c, \rho$ independent of $\lambda \in U$. 


\subsection{Distortion properties}

We start with the following well-known result.

Lemma 4.6 (Koebe's Distortion Theorem). There exists a constant $K_{2} \geq 1 \mathrm{such}$ that if $D \subset \mathbb{C}$ is a geometric disk, and $g: D \rightarrow \mathbb{C}$ is a univalent holomorphic function, then for all $w, z \in \frac{1}{2} D$

$$
1-K_{2}|z-w| \leq \frac{\left|g^{\prime}(w)\right|}{\left|g^{\prime}(z)\right|} \leq 1+K_{2}|z-w|
$$

or equivalently

$$
|| g^{\prime}(w)|-| g^{\prime}(z)|| \leq K_{2}\left|g^{\prime}(z)\right||z-w| .
$$

Since $\log (1+x) \leq x$ for all $x \geq-1$, it follows from the first inequality above that

$$
|\log | g^{\prime}(w)|-\log | g^{\prime}(z)|| \leq K_{2}|z-w| \text {. }
$$

Given $T>0$ we denote by $\mathcal{K}_{T}$ the class of all univalent holomorphic functions whose domains are geometric disks in $\mathbb{C} \backslash D(0, T)$ with Euclidean radii $\leq 1$ and whose ranges are contained in $\mathbb{C} \backslash D(0, T)$. We shall prove the following Lemma in which we write again

$$
\left|g^{\prime}(z)\right|_{\tau}=\left|g^{\prime}(z)\right| \frac{|z|^{\tau}}{|g(z)|^{\tau}}
$$

the derivative of $g$ with respect to the Riemannian metric $\tau$ (given in (3.2)).

Lemma 4.7. There exists a constant $K=K_{\tau, T} \geq 1$ such that if $g: D \rightarrow \mathbb{C}$ belongs to $\mathcal{K}_{T}$, then for all $z, w \in \frac{1}{2} D$,

$$
\left.|\log | g^{\prime}(w)\right|_{\tau}-\log \left|g^{\prime}(z)\right|_{\tau}\left|\leq K\left(1+\left|g^{\prime}(z)\right|\right)\right| z-w \mid
$$

and

$$
\left|\frac{\left|g^{\prime}(w)\right|_{\tau}}{\left|g^{\prime}(z)\right|_{\tau}}-1\right| \leq K\left(1+\left|g^{\prime}(z)\right|\right)|z-w|
$$

ProOF. Rewrite (4.6) in the logarithmic form:

$$
\log \left|g^{\prime}(\xi)\right|_{\tau}=\log \left|g^{\prime}(\xi)\right|+\tau \log |\xi|-\tau \log |g(\xi)| .
$$

Then, using in turn the second part of Lemma 4.6.

$$
\begin{aligned}
& \left.|\log | g^{\prime}(w)\right|_{\tau}-\log \left|g^{\prime}(z)\right|_{\tau} \mid= \\
& \quad=|\log | g^{\prime}(w)|-\log | g^{\prime}(z)|+\tau \log (|w| /|z|)+\tau \log (|g(z)| /|g(w)|)| \\
& \quad \leq|\log | g^{\prime}(w)|-\log | g^{\prime}(z)||+\tau \log \left(1+\frac{|w-z|}{|z|}\right)+\tau \log \left(1+\frac{|g(z)-g(w)|}{|g(w)|}\right) \\
& \quad \leq K_{2}|z-w|+\frac{\tau}{|z|}|w-z|+\frac{\tau}{|g(w)|}|g(z)-g(w)| \\
& \quad \leq K_{2}|z-w|+\tau T^{-1}|w-z|+\tau T^{-1}\left(1+K_{2}|z-w|\right)\left|g^{\prime}(z)\right||z-w| \\
& \quad \leq\left(\left(K_{2}+\tau T^{-1}\right)+\left(\tau T^{-1}\left(1+2 K_{2}\right)\left|g^{\prime}(z)\right|\right)|z-w|\right. \\
& \quad \leq\left(K_{2}+\tau T^{-1}\left(1+2 K_{2}\right)\right)\left(1+\left|g^{\prime}(z)\right|\right)|w-z|
\end{aligned}
$$


and the first formula constituting our lemma is proved. Applying to it the Mean Value Theorem, we get with some $A \in\left[\min \left\{\left|g^{\prime}(w)\right|_{\tau},\left|g^{\prime}(z)\right|_{\tau}\right\}, \max \left\{\left|g^{\prime}(w)\right|_{\tau},\left|g^{\prime}(z)\right|_{\tau}\right\}\right]$, that

$$
\left.A^{-1}|| g^{\prime}(w)\right|_{\tau}-\left|g^{\prime}(z)\right|_{\tau}\left|\leq K\left(1+\left|g^{\prime}(z)\right|\right)\right| z-w \mid .
$$

So, invoking the first part of Theorem 4.6. we get

$$
\left.|| g^{\prime}(w)\right|_{\tau}-\left|g^{\prime}(z)\right|_{\tau}\left|\leq K\left(1+K_{2}\right)\right| g^{\prime}(z)\left|\left(1+\left|g^{\prime}(z)\right|\right)\right| z-w \mid,
$$

and the second part of our lemma is also proved with appropriately large $K \geq 1$.

Denote by $\mathcal{K}_{T}^{M}$ the subclass of $\mathcal{K}_{T}$ consisting of those functions $g$ for which $\left\|g^{\prime}\right\|_{\infty} \leq$ $M$. Lemma 4.7 takes then the following form.

Corollary 4.8. There exists a constant $K=K_{\tau, T, M} \geq 1$ such that if $g: D \rightarrow \mathbb{C}$ is in $\mathcal{K}_{T}^{M}$, then for all $z, w \in \frac{1}{2} D$,

$$
\left.|\log | g^{\prime}(w)\right|_{\tau}-\log \left|g^{\prime}(z)\right|_{\tau}|\leq K| z-w \mid
$$

and

$$
\left|\frac{\left|g^{\prime}(w)\right|_{\tau}}{\left|g^{\prime}(z)\right|_{\tau}}-1\right| \leq K|z-w|
$$

Here is the typical example of application of the above distortion lemmas.

Lemma 4.9. Let $f: \mathbb{C} \rightarrow \hat{\mathbb{C}}$ be a hyperbolic meromorphic function and let $\delta=$ $\delta(f)>0$. For every $\tau>0$ there exists a constant $K_{\tau} \geq 1$ such that for every integer $n \geq 0$, every $w \in \mathcal{J}(f)$, every $z \in f^{-n}(w)$ and all $x, y \in D(w, \delta)$, we have that

$$
K_{\tau}^{-1} \leq \frac{\left|\left(f_{z}^{-n}\right)^{\prime}(y)\right|_{\tau}}{\left|\left(f_{z}^{-n}\right)^{\prime}(x)\right|_{\tau}} \leq K_{\tau}
$$

Here and in the rest of the text $f_{z}^{-n}$ signifies the inverse branch of $f^{n}$ defined near $f^{n}(z)$ mapping $f^{n}(z)$ back to $z$.

\subsection{Hölder functions and dynamical Hölder property}

Let $f: \mathbb{C} \rightarrow \hat{\mathbb{C}}$ be a meromorphic hyperbolic function and denote $\delta=\delta(f)$ the constant given by the topological hyperbolicity of $f$. Fix $\beta \in(0,1]$. Given $h: \mathcal{J}(f) \rightarrow \mathbb{C}$, let

$$
v_{\beta}(h)=\sup \left\{\frac{|h(y)-h(x)|}{|y-x|^{\beta}} \text { for all } x, y \in \mathcal{J}(f) \text { with } 0<|y-x| \leq \delta\right\},
$$

be the $\beta$-variation of the function $h$. Any function with bounded $\beta$-variation will be called $\beta$-Hölder or simply Hölder continuous if we do not want to specify the exponent of Hölder continuity. Let

$$
\|h\|_{\beta}=v_{\beta}(h)+\|h\|_{\infty} .
$$

be the norm of the space

$$
\mathrm{H}_{\beta}=\mathrm{H}_{\beta}(\mathcal{J}(f))=\left\{h: \mathcal{J}(f) \rightarrow \mathbb{C}:\|h\|_{\beta}<\infty\right\} .
$$

Any member of $\mathrm{H}_{\beta}$ will be called a bounded $\beta$-Hölder continuous function. The function $\log \left|f^{\prime}\right|_{\tau}$ is not necessary Hölder continuous which is the reason for the 
following slightly more general form of Hölder continuity. In order to introduce it consider $w \in \mathcal{J}(f)$ and denote the $\beta$-variation of a function $h: \mathcal{J}(f) \cap D(w, \delta) \rightarrow \mathbb{C}$ by

$$
V_{\beta, w}(h)=\sup \left\{\frac{|h(x)-h(y)|}{|x-y|^{\beta}} ; x, y \in \mathcal{J}(f) \cap D(w, \delta)\right\} .
$$

A function $h: \mathcal{J}(f) \rightarrow \mathbb{C}$ is called $\beta$-weakly Hölder continuous if $V_{\beta, w}\left(h \circ f_{a}^{-1}\right)$ is bounded uniformly in $w \in \mathcal{J}(f)$ and $a \in f^{-1}(w)$. Denote

$$
V_{\beta}(h)=\sup _{w \in \mathcal{J}(f)} \sup _{a \in f^{-1}(w)} V_{\beta, w}\left(h \circ f_{a}^{-1}\right) .
$$

and let $\mathrm{H}_{\beta}^{w}$ be the space of bounded weakly $\beta$-Hölder continuous functions equipped with the norm

$$
\||| h \mid\|_{\beta}=V_{\beta}(h)+\|h\|_{\infty} .
$$

Both spaces $\mathrm{H}_{\beta}$, $\mathrm{H}_{\beta}^{w}$ endowed with their respective norms are Banach spaces densely contained in the space of all bounded continuous complex valued functions $C_{b}$ with respect to the $\|\cdot\|_{\infty}$ norm.

Lemma 4.10. If $f: \mathbb{C} \rightarrow \hat{\mathbb{C}}$ is a hyperbolic meromorphic function, then

(1) $\mathrm{H}_{\beta} \subset \mathrm{H}_{\beta}^{w}$ with $\||\cdot|\|_{\beta} \preceq\|\cdot\|_{\beta}$ and

(2) $\log \left|f^{\prime}\right|_{\tau}$ is weakly 1 -Hölder continuous.

PROOF. The inclusion of the spaces with control of the respective norms results from the expanding property of $f$. The second assertion is a consequence of the distortion Lemma 4.7

Given $n \geq 0$, let

$$
S_{n} h(z)=h(z)+h(f(z))+\cdots+h\left(f^{n-1}(z)\right) .
$$

Lemma 4.11. For every $\beta>0$ there exists $c_{\beta}>0$ such that if $h: \mathcal{J}(f) \rightarrow \mathbb{C}$ is a weakly $\beta$-Hölder function, then

$$
\left|S_{n} h\left(f_{v}^{-n}(y)\right)-S_{n} h\left(f_{v}^{-n}(x)\right)\right| \leq c_{\beta} V_{\beta}(h)|y-x|^{\beta}
$$

for all $n \geq 1$, all $x, y \in \mathcal{J}(f)$ with $|x-y| \leq \delta$ and all $v \in f^{-n}(x)$.

Proof. Denote $a=f_{v}^{-n}(x)$ and $b=f_{v}^{-n}(y)$. For $k=0, \ldots, n-1$ we have that $\left|h\left(f^{k}(b)\right)-h\left(f^{k}(a)\right)\right| \leq V_{\beta}(h)\left|f^{k+1}(b)-f^{k+1}(a)\right|^{\beta} \leq c^{-\beta} V_{\beta}(h) \gamma^{(k+1-n) \beta}|y-x|^{\beta}$ by Koebe distortion (cf. (4.6)) and the expanding property. Since $\gamma>1$,

$$
\left|S_{n} h(b)-S_{n} h(a)\right| \leq \frac{c^{-\beta}}{1-\gamma^{-\beta}} V_{\beta}(h)|y-x|^{\beta}
$$

which proves the lemma.

A simple application of the Mean Value Theorem to the function $z \mapsto e^{z}$ together with the previous Lemma 4.11 gives the following.

Lemma 4.12. Let $h: \mathcal{J}(f) \rightarrow \mathbb{C}$ be a weakly $\beta$-Hölder continuous function. Then there exists a constant $c$ depending only on $\beta$ and the variation $V_{\beta}(h)$ such that

$$
\left|\exp \left(S_{n} h\left(f_{v}^{-n}(y)\right)\right)-\exp \left(S_{n} h\left(f_{v}^{-n}(x)\right)\right)\right| \leq c\left|\exp \left(S_{n} h\left(f_{v}^{-n}(x)\right)\right)\right||y-x|^{\beta}
$$

for all $n \geq 1$, all $x, y \in \mathcal{J}(f)$ with $|x-y| \leq \delta$ and all $v \in f^{-n}(x)$. 


\section{CHAPTER 5}

\section{Perron-Frobenius Operators and Generalized Conformal Measures}

In this chapter we develope and generalize MyU2 building up the thermodynamical formalism for a very general class potentials. The culminating point is the proof and formulation of Theorem 5.15. Throughout the whole chapter we suppose that $f: \mathbb{C} \rightarrow \hat{\mathbb{C}}$ is dynamically semi-regular, i.e. a hyperbolic meromorphic function of finite order $\rho$ that satisfies the growth condition (2.4).

\subsection{Tame potentials}

The class of potentials we have in mind is the following.

Definition 5.1. A function $\phi: \mathcal{J}(f) \rightarrow \mathbb{C}$ is called tame (or, more precisely, $(t, \beta)$-tame) if there is $t>\frac{\rho}{\alpha}$ and a bounded weakly $\beta$-Hölder continuous function $h: \mathcal{J}(f) \rightarrow \mathbb{C}$ such that

$$
\phi(z)=-t \log \mid f^{\prime}(z){\underline{\alpha_{2}}}+h(z) \quad, \quad z \in \mathcal{J}(f) .
$$

A function $\phi$ that satisfies this definition but with arbitrary $t \in \mathbb{R}$ (or with $t>0$ ) is called loosely tame (respectively $0^{+}$-tame). We also use these notions of tameness for complex-valued functions.

Note that in this definition we have taken the derivatives with respect to a fixed metric (depending on $f$ only). But for any tame $\Phi=-t \log \left|f^{\prime}\right|_{\underline{\alpha}_{2}}+h$ we can make a cohomologous change of potential and (without changing the name) switch to

$$
\phi(z)=-t \log \left|f^{\prime}(z)\right|_{\tau}+h(z)=\Phi(z)+\left(\underline{\alpha}_{2}-\tau\right) t(\log |z|-\log |f(z)|),
$$

where

$$
\tau \in\left(0, \underline{\alpha}_{2}\right) \text { is chosen such that } t>\frac{\rho}{\hat{\tau}}=\frac{\rho}{\alpha_{1}+\tau}>\frac{\rho}{\alpha} .
$$

Since cohomologous functions share the same Gibbs (or equilibrium) states (see Theorem 6.19) and since the whole point of this work is to study ergodic and geometric properties of Gibbs states, we can and do work with $\phi$ as well as with $\Phi$. In other words, we can work with the metric $d \tau$ we like to and we will indeed always take $\tau$ depending on $t>\rho / \alpha$ such that (5.1) is satisfied.

Now we collect some basic properties for these potentials. Notice first that every loosely tame function is a (usually unbounded) weakly Hölder continuous function (cf. Lemma 4.10). The distortion properties given in Lemma 4.11 and in Lemma 4.12 yield the following. 
Lemma 5.2. For every loosely tame potential $\phi=-t \log \left|f^{\prime}\right|_{\tau}+h: \mathcal{J}(f) \rightarrow \mathbb{C}$, $h \in \mathrm{H}_{\beta}^{w}$, there is $c_{\beta}>0$ such that

$$
\left|S_{n} \phi\left(f_{v}^{-n}(y)\right)-S_{n} \phi\left(f_{v}^{-n}(x)\right)\right| \leq c_{\beta} V_{\beta}(h)|y-x|^{\beta}
$$

for all $n \geq 0$, all $z \in \mathcal{J}(f)$, all $v \in f^{-n}(z)$ and all $x, y \in D(z, \delta)$. Here $K \geq 1$ is the distortion constant from Lemma 4.7 .

We also have a dynamically Hölder property for loosely tame potentials.

Lemma 5.3. If $\phi: \mathcal{J}(f) \rightarrow \mathbb{C}$ is a $(t, \beta)$-loosely tame potential, then there exists $c=c_{\phi}>0$ depending only on $\beta$ and $V_{\beta}(\phi)$ such that

$$
\left|\exp \left(S_{n} \phi\left(f_{v}^{-n}(y)\right)\right)-\exp \left(S_{n} \phi\left(f_{v}^{-n}(x)\right)\right)\right| \leq c\left|\exp \left(S_{n} \phi\left(f_{v}^{-n}(x)\right)\right)\right||y-x|^{\beta}
$$

for all $n \geq 0$, all $z \in \mathcal{J}(f)$, all $v \in f^{-n}(z)$ and all $x, y \in D(z, \delta)$.

As an immediate consequence of this lemma, applied with $n=1$, and the left-hand side of (3.4), we get the following.

Corollary 5.4. If $\phi: \mathcal{J}(f) \rightarrow \mathbb{C}$ is a $(t, \beta)$-tame potential with $t>0$ and $\beta \in(0,1]$, then $e^{\phi} \in \mathrm{H}_{\beta}$.

\subsection{Growth condition and cohomological Perron-Frobenius operator}

Throughout the rest of the chapter we consider $\phi=-t \log \left|f^{\prime}\right|_{\tau}+h$ a tame potential with real-valued function $h \in \mathrm{H}_{\beta}^{w}$. The transfer operator $\mathcal{L}_{\phi}$ associated to a tame potential $\phi$ is defined by

$$
\mathcal{L}_{\phi} g(w)=\sum_{z \in f^{-1}(w)} g(z) \exp (\phi(z))=\sum_{z \in f^{-1}(w)} g(z)\left|f^{\prime}(z)\right|_{\tau}^{-t} \exp (h(z))
$$

where $g$ is a function of the Banach space $C_{b}(\mathcal{J}(f))$ of bounded continuous functions on $\mathcal{J}(f)$. If we deal with (geometric) potentials $\phi=-t \log \left|f^{\prime}\right|_{\tau}$ then we also use the notation $\mathcal{L}_{t}$ for $\mathcal{L}_{-t \log \left|f^{\prime}\right|_{\tau}}$. Note that for $n \geq 1$

$$
\mathcal{L}_{\phi}^{n} g(w)=\sum_{z \in f^{-n}(w)} g(z) \exp \left(S_{n} \phi(z)\right)
$$

and

$$
\mathcal{L}_{\phi}^{n}\left(\psi_{1} \cdot \psi_{2} \circ f^{n}\right)=\psi_{2} \mathcal{L}_{\phi}^{n} \psi_{1}
$$

for all functions $\psi_{1}, \psi_{2}: \mathcal{J}(f) \rightarrow \mathbb{C}$. We also have

$$
\left|\mathcal{L}_{\phi} g\right| \leq e^{\|h\|_{\infty}} \mathcal{L}_{t}|g| \quad \text { for all } g \in C_{b}(\mathcal{J}(f))
$$

and, in particular,

$$
\begin{aligned}
\mathcal{L}_{\phi} \mathbb{1}(w) & \preceq \mathcal{L}_{t} \mathbb{1}(w)=\sum_{z \in f^{-1}(w)}\left|f^{\prime}(z)\right|_{\tau}^{-t}=\sum_{z \in f^{-1}(w)}\left|f^{\prime}(z)\right|^{-t}|z|^{-\tau t}|f(z)|^{\tau t} \\
& \leq \frac{\kappa^{t}}{|w|^{t\left(\underline{\alpha}_{2}-\tau\right)}} \sum_{z \in f^{-1}(w)}|z|^{-\hat{\tau} t}
\end{aligned}
$$

because $f$ satisfies the growth condition (2.4). From the Borel-Picard Theorem 3.5 we see that the last sum is finite. From our standard assumption $0 \notin \mathcal{J}(f)$ and 
since $t \hat{\tau}>\rho$ we get that the uniform control of this last sum given in Proposition 3.6 applies and explains that there are $\mathcal{M}_{\phi}, M_{\phi}>0$ such that

$$
\mathcal{L}_{\phi} \mathbb{1}(w) \leq \frac{\mathcal{M}_{\phi}}{|w|^{t\left(\underline{\alpha}_{2}-\tau\right)}} \leq M_{\phi} \quad \text { for all } w \in \mathcal{J}(f) .
$$

Put

$$
M_{u}:=M_{-u \log \left|f^{\prime}\right|_{\tau}} \text { if } u>\rho / \hat{\tau} .
$$

This uniform control secures continuity of the operator $\mathcal{L}_{\phi}$ on the Banach space $C_{b}(\mathcal{J}(f))$ of bounded continuous functions endowed with the standard supremum norm. We therefore have

Theorem 5.5. Assume that $f: \mathbb{C} \rightarrow \hat{\mathbb{C}}$ is dynamically semi-regular. Then, for every tame potential $\phi$, the transfer operator $\mathcal{L}_{\phi}$ is well defined and acts continuously on the Banach space $C_{b}(\mathcal{J}(f))$.

We conclude this part with the following two observations.

Lemma 5.6. Let $\delta=\delta(f)>0$, let $\phi=-t \log \left|f^{\prime}\right|_{\sigma}+h$ be a tame potential and let $c=c\left(\beta, V_{\beta}(\phi)\right)$ be the constant given in Lemma 5.3. Then

$$
\left|\mathcal{L}_{\phi}^{n} \mathbb{1}\left(w_{2}\right)-\mathcal{L}_{\phi}^{n} \mathbb{1}\left(w_{1}\right)\right| \leq c \mathcal{L}_{\phi}^{n} \mathbb{1}\left(w_{1}\right)\left|w_{1}-w_{2}\right|^{\beta}
$$

and

$$
\mathcal{L}_{\phi}^{n} \mathbb{1}\left(w_{1}\right) \leq\left(1+c\left|w_{1}-w_{2}\right|^{\beta}\right) \mathcal{L}_{\phi}^{n} \mathbb{1}\left(w_{2}\right)
$$

for every $n \geq 0$ and for all $w_{1}, w_{2} \in \mathcal{J}(f)$ with $\left|w_{1}-w_{2}\right|<\delta$.

Proof. For $w_{1}, w_{2} \in \mathcal{J}(f)$ with $\left|w_{1}-w_{2}\right|<\delta$ we have

$$
\begin{aligned}
\left|\mathcal{L}_{\phi}^{n} \mathbb{1}\left(w_{1}\right)-\mathcal{L}_{\phi}^{n} \mathbb{1}\left(w_{2}\right)\right| & \leq \sum_{a \in f^{-n}\left(w_{2}\right)}\left|e^{S_{n} \phi\left(f_{a}^{-n}\left(w_{1}\right)\right)}-e^{S_{n} \phi(a)}\right| \\
& \preceq \sum_{a \in f^{-n}\left(w_{2}\right)} e^{S_{n} \phi(a)}\left|w_{2}-w_{1}\right|^{\beta} \\
& =\mathcal{L}_{\phi}^{n} \mathbb{1}\left(w_{2}\right)\left|w_{2}-w_{1}\right|^{\beta}
\end{aligned}
$$

because of Lemma 5.3

Lemma 5.7. For every tame potential $\phi$ and for every $R>0$ there exists $K_{\phi, R} \geq 1$ such that

$$
\mathcal{L}_{\phi}^{n} \mathbb{1}\left(w_{1}\right) \leq K_{\phi, R} \mathcal{L}_{\phi}^{n} \mathbb{1}\left(w_{2}\right)
$$

for every $n \geq 0$ and for all $w_{1}, w_{2} \in \mathcal{J}(f) \cap \bar{D}(0, R)$.

Proof. Let $\delta=\delta(f)>0$ and set $K=\mathcal{J}(f) \cap \bar{D}(0, R)$. Lemma 4.2 asserts that there is $N=N(R) \geq 0$ such that for any $w_{1}, w_{2} \in K$ there is $z \in D\left(w_{1}, \delta\right)$ with $f^{N}(z)=w_{2}$. Hence $\mathcal{L}_{\phi}^{n+N} \mathbb{1}\left(w_{2}\right) \geq e^{S_{N} \phi(z)} \mathcal{L}_{\phi}^{n} \mathbb{1}(z)$. It follows from the previous Lemma 5.6 that there is $C=C(\phi) \geq 1$ such that

$$
\mathcal{L}_{\phi}^{n} \mathbb{1}\left(w_{1}\right) \leq C \mathcal{L}_{\phi}^{n} \mathbb{1}(z) \leq C e^{-S_{N} \phi(z)} \mathcal{L}_{\phi}^{N+n} \mathbb{1}\left(w_{2}\right) \leq C e^{-S_{N} \phi(z)} M_{\phi}^{N} \mathcal{L}_{\phi}^{n} \mathbb{1}\left(w_{2}\right)
$$

for every $n \geq 0$. The assertion follows because the function $\exp \left(-S_{N}\right)$ is welldefined and continuous on the compact set $\mathcal{J}(f) \cap \bar{D}(0, R+\delta) \cap f^{-N}(K)$, and therefore it is bounded there. 


\subsection{Topological pressure and existence of conformal measures}

We first need the notion of topological pressure. Let us start with the following simple observation.

Lemma 5.8. The number $\lim \sup _{n \rightarrow \infty} \frac{1}{n} \log \mathcal{L}_{\phi}^{n} 1(w)$ is independent of $w \in \mathcal{J}(f)$.

Proof. Let $w_{1}, w_{1} \in \mathcal{J}(f)$ be any two points and denote again $\delta=\delta(f)$. Lemma [5.7yields that there is $k=k\left(\phi,\left|w_{1}\right|,\left|w_{2}\right|\right) \geq 0$ such that

$$
\mathcal{L}_{\phi}^{n} \mathbb{1}\left(w_{1}\right) \leq k \mathcal{L}_{\phi}^{n} \mathbb{1}\left(w_{2}\right) \quad \text { for every } n \geq 0 .
$$

Therefore

$$
\limsup _{n \rightarrow \infty} \frac{1}{n} \log \mathcal{L}_{\phi}^{n} \mathbb{1}\left(w_{1}\right) \leq \limsup _{n \rightarrow \infty} \frac{1}{n} \log \mathcal{L}_{\phi}^{n} \mathbb{1}\left(w_{2}\right)
$$

which shows the lemma.

Definition 5.9. The topological pressure of $\phi$ is

$$
\mathrm{P}(\phi)=\mathrm{P}(\phi, w)=\limsup _{n \rightarrow \infty} \frac{1}{n} \log \mathcal{L}_{\phi}^{n} \mathbb{1}(w), w \in \mathcal{J}(f) .
$$

We will see later (Corollary [5.18) that the sequence $\frac{1}{n} \log \mathcal{L}_{\phi}^{n} \mathbb{1}(w), w \in \mathcal{J}(f)$, actually converges which permits then to define the pressure $\mathrm{P}(\phi)$ as the limit of this sequence.

Further properties of transfer operators $\mathcal{L}_{\phi}$ rely on the existence of conformal measures.

Definition 5.10. A probability measure $m_{\phi}$ is called $\rho e^{-\phi}$-conformal if one of the following equivalent properties holds:

1) For every $E \subset \mathcal{J}(f)$ such that $f_{\mid E}$ is injective we have

$$
m_{\phi}(f(E))=\int_{E} \rho e^{-\phi} d m_{\phi}
$$

2) $m_{\phi}$ is an eigenmeasure of the adjoint $\mathcal{L}_{\phi}^{*}$ of the transfer operator $\mathcal{L}_{\phi}$ with eigenvalue $\rho$ :

$$
\mathcal{L}_{\phi}^{*} m_{\phi}=\rho m_{\phi} .
$$

The equivalence between these conditions is a straightforward calculation (see for example [DU1] where the finiteness of the partition can be replaced by its countability).

If the Hölder function $h \equiv 0$ then we deal with geometric potentials $\phi=$ $-t \log \left|f^{\prime}\right|_{\sigma}$ and we simply denote by $m_{t}$ the conformal measure $m_{-t \log \left|f^{\prime}\right|_{\sigma}}$. Note that then the measure $m_{t}^{e}$, the Euclidean version of $m_{t}$, defined by the requirement that $d m_{t}^{e}(z)=|z|^{\alpha_{2} t} d m_{t}(z)$ is $\rho\left|f^{\prime}\right|^{t}$-conformal (but $m_{t}^{e}$ is not necessary a finite measure) in the sense that

$$
m_{t}^{e}(f(A))=\int_{A} \rho\left|f^{\prime}\right|^{t} d m_{t}^{e}
$$

Our aim now is to construct conformal measures for a given tame function $\phi$ with the precise information on the conformal factor, namely we want to have $\rho=$ $e^{\mathrm{P}(\phi)}$. In the case the conformal factor $\rho=1$ or, equivalently, if the topological pressure $\mathrm{P}(\phi)=0$, and if the potential is $\phi=-t \log \left|f^{\prime}\right|_{\sigma}$ these measures are simply called $t$-conformal. In $[\mathbf{S u}]$ Sullivan has proved that every rational function admits a probability conformal measure. As it is shown in $\mathbf{M y \mathbf { U } 2}$, in the case of 
meromorphic functions the situation is not that far apart. All what you need for the existence of a conformal measure is the rapid derivative growth; no hyperbolicity is necessary 1 . We adapt here the very general construction of $\mathbf{M y U 2}$ in order to get

Theorem 5.11. If $f: \mathbb{C} \rightarrow \hat{\mathbb{C}}$ is a meromorphic function of finite order with nonempty Fatou set satisfying the growth condition (2.4), then for every tame potential $\phi$ there exists a Borel probability $e^{\mathrm{P}(\phi)} e^{-\phi}$-conformal measure $m_{\phi}$ on $\mathcal{J}(f)$.

The rest of this section is devoted to the proof of Theorem 5.11. We may again assume without loss of generality that $0 \notin \mathcal{J}(f)$. Fix $w \in \mathcal{J}(f)$. Observe that the transition parameter for the series

$$
\Sigma_{s}=\sum_{n=1}^{\infty} e^{-n s} \mathcal{L}_{\phi}^{n} \mathbb{1}(w)
$$

is the topological pressure $\mathrm{P}(\phi)$. In other words, $\Sigma_{s}=+\infty$ for $s<\mathrm{P}(\phi)$ and $\Sigma_{s}<$ $\infty$ for $s>\mathrm{P}(\phi)$. We assume that we are in the divergence case, e.g. $\Sigma_{\mathrm{P}(\phi)}=\infty$. For the convergence type situation the usual modifications have to be done (see DU1 for details). For $s>\mathrm{P}(\phi)$, put

$$
\nu_{s}=\frac{1}{\Sigma_{s}} \sum_{n=1}^{\infty} e^{-n s}\left(\mathcal{L}_{\phi}^{n}\right)^{*} \delta_{w} .
$$

The following lemma follows immediately from definitions.

Lemma 5.12. The following properties hold:

(1) For every $g \in \mathcal{C}_{b}(\mathbb{C})$ we have

$$
\int g d \nu_{s}=\frac{1}{\Sigma_{s}} \sum_{n=1}^{\infty} e^{-n s} \int \mathcal{L}_{\phi}^{n} g d \delta_{w}=\frac{1}{\Sigma_{s}} \sum_{n=1}^{\infty} e^{-n s} \mathcal{L}_{\phi}^{n} g(w) .
$$

(2) $\nu_{s}$ is a probability measure.

$$
\frac{1}{e^{s}} \mathcal{L}_{\phi}^{*} \nu_{s}=\frac{1}{\Sigma_{s}} \sum_{n=1}^{\infty} e^{-(n+1) s}\left(\mathcal{L}_{\phi}^{n+1}\right)^{*} \delta_{w}=\nu_{s}-\frac{1}{\Sigma_{s}} \frac{\mathcal{L}_{\phi}^{*} \delta_{w}}{e^{s}}
$$

The key ingredient of the proof of Theorem 5.11 is to show that the family $\left(\nu_{s}\right)_{s>P(\phi)}$ of Borel probability (see Lemma $5.12(2))$ measures on $\mathbb{C}$ is tight and then to apply Prokhorov's Theorem. In order to accomplish this we put

$$
U_{R}=\{z \in \mathbb{C}:|z|>R\}
$$

and start with the following observation.

Lemma 5.13. For every $(t, \beta)$-tame potential $\phi$ there is $C=C(\phi, \tau)>0$ such that

$$
\mathcal{L}_{\phi}\left(\mathbb{1}_{U_{R}}\right)(w) \leq \frac{C}{R^{\hat{\tau} \gamma}} \text { for every } w \in \mathcal{J}(f),
$$

where $\gamma=\frac{t-\rho / \hat{\tau}}{2}$.

\footnotetext{
${ }^{1}$ Since $f$ is not supposed to be hyperbolic $\mathcal{F}_{f}=\emptyset$ may occur. But then the Lebesgue measure is 2-conformal.
} 
Proof. We have that $\phi=-t \log \left|f^{\prime}\right|_{\tau}+h$ with $t>\frac{\alpha}{\rho}$ and $h$ a bounded Hölder continuous function. From the growth condition (2.4) and Proposition 3.6, similarly as (5.4), we get for every $w \in \mathcal{J}(f)$ that

$$
\begin{aligned}
\mathcal{L}_{t}\left(\mathbb{1}_{U_{R}}\right)(w) & =\sum_{z \in f^{-1}(w) \cap U_{R}} e^{h(z)}\left|f^{\prime}(z)\right|_{\tau}^{-t} \leq \frac{\kappa^{t} e^{\|h\|_{\infty}}}{|w|^{t\left(\underline{\alpha}_{2}-\tau\right)}} \sum_{z \in f^{-1}(w) \cap U_{R}}|z|^{-\hat{\tau} t} \\
& \leq \frac{\kappa^{t} e^{\|h\|_{\infty}}}{T^{t\left(\underline{\alpha}_{2}-\tau\right)}} \frac{1}{R^{\hat{\tau} \gamma}} \sum_{z \in f^{-1}(w)}|z|^{-(\rho+\hat{\tau} \gamma)} \leq \frac{C(\phi, \tau)}{R^{\hat{\tau} \gamma}}
\end{aligned}
$$

Now we are ready to prove the tightness we have already announced. We recall that this means that

$$
\forall \varepsilon>0 \quad \exists R>0 \text { such that } \nu_{s}\left(U_{R}\right) \leq \varepsilon \text { for all } s>\mathrm{P}(\phi) .
$$

Lemma 5.14. The family $\left(\nu_{s}\right)_{s>\mathrm{P}(\phi)}$ of Borel probability measures on $\mathbb{C}$ is tight and, more precisely, there is $L>0$ and $\delta>0$ such that

$$
\nu_{s}\left(U_{R}\right) \leq L R^{-\delta} \text { for all } R>0 \text { and } s>\mathrm{P}(\phi) .
$$

Proof. The first observation is that

$$
\begin{aligned}
\mathcal{L}_{\phi}^{n+1}\left(\mathbb{1}_{U_{R}}\right)(w) & =\sum_{y \in f^{-n}(w)} \sum_{z \in f^{-1}(y) \cap U_{R}} e^{h(z)}\left|f^{\prime}(z)\right|_{\tau}^{-t} e^{S_{n} h(y)}\left|\left(f^{n}\right)^{\prime}(y)\right|_{\tau}^{-t} \\
& =\sum_{y \in f^{-n}(w)} e^{S_{n} h(y)}\left|\left(f^{n}\right)^{\prime}(y)\right|_{\tau}^{-t} \mathcal{L}_{\phi}\left(\mathbb{1}_{U_{R}}\right)(y) \leq \frac{C}{R^{\hat{\gamma} \gamma}} \mathcal{L}_{\phi}^{n} \mathbb{1}(w) .
\end{aligned}
$$

where the last inequality follows from Lemma 5.13. Therefore, for every $s>\mathrm{P}(\phi)$, we get that

$$
\begin{aligned}
\nu_{s}\left(U_{R}\right) & =\frac{1}{\Sigma_{s}} \sum_{n=1}^{\infty} e^{-n s} \mathcal{L}_{\phi}^{n}\left(\mathbb{1}_{U_{R}}\right)(w) \leq \frac{C}{R^{\hat{\tau} \gamma}} \frac{1}{\Sigma_{s}} \sum_{n=1}^{\infty} e^{-n s} \mathcal{L}_{\phi}^{n-1} \mathbb{1}(w) \\
& =\frac{C}{R^{\hat{\tau} \gamma}} \frac{1}{e^{s}} \frac{1}{\Sigma_{s}}\left(1+\sum_{n=1}^{\infty} e^{-n s} \mathcal{L}_{\phi}^{n} \mathbb{1}(w)\right) \leq \frac{2 C}{e^{\mathrm{P}(\phi)}} \frac{1}{R^{\hat{\tau} \gamma}} .
\end{aligned}
$$

This shows Lemma 5.14 and the tightness of the family $\left(\nu_{s}\right)_{s>\mathrm{P}(\phi)}$.

Now, choose a sequence $\left\{s_{j}\right\}_{j=1}^{\infty}, s_{j}>\mathrm{P}(\phi)$, converging down to $\mathrm{P}(\phi)$. In view of Prokhorov's Theorem and Lemma 5.14 passing to a subsequence, we may assume without loss of generality that the sequence $\left\{\nu_{s_{j}}\right\}_{j=1}^{\infty}$ converges weakly to a Borel probability measure $m_{\phi}$ on $\mathcal{J}(f)$. It follows from Lemma 5.12 and the divergence property of $\Sigma_{\mathrm{P}(\phi)}$ that $\mathcal{L}_{\phi}^{*} m_{\phi}=e^{\mathrm{P}(\phi)} m_{\phi}$. The proof of Theorem 5.11 is complete.

\subsection{Thermodynamical Formalism}

We can now establish the following main result of this chapter.

Theorem 5.15. If $f: \mathbb{C} \rightarrow \hat{\mathbb{C}}$ is a dynamically semi-regular meromorphic function, then for every tame potential $\phi$ the following are true. 
(1) The topological pressure $\mathrm{P}(\phi)=\lim _{n \rightarrow \infty} \frac{1}{n} \log \mathcal{L}_{\phi}^{n} \mathbb{1}(w)$ exists and is independent of $w \in \mathcal{J}(f)$.

(2) There exists a unique $\rho e^{-\phi}$-conformal measure $m_{\phi}$ and necessarily $\rho=$ $e^{\mathrm{P}(\phi)}$. Also, there exists a unique Gibbs state $\mu_{\phi}$, i.e. $\mu_{\phi}$ is $f$-invariant and equivalent to $m_{\phi}$.

(3) Both measures $m_{\phi}$ and $\mu_{\phi}$ are ergodic and supported on the radial (or conical) Julia set $\mathcal{J}_{r}(f)$.

(4) The density $\rho_{\phi}=d \mu_{\phi} / d m_{\phi}$ is a nowhere vanishing continuous and bounded function on the Julia set $\mathcal{J}(f)$.

The remaining part of this chapter is devoted to the proof of this key result.

5.4.1. Existence of the Gibbs (or equilibrium) states. Let us start by making the following observation which is an immediate consequence of the choice of the $\tau$-metric (see (5.5)).

Lemma 5.16. We have $\lim _{w \rightarrow \infty} \mathcal{L}_{\phi} \mathbb{1}(w)=0$.

We consider now the normalized transfer operator

$$
\hat{\mathcal{L}}_{\phi}=e^{-\mathrm{P}(\phi)} \mathcal{L}_{\phi}
$$

and establish the following important uniform estimates.

Proposition 5.17. There exists $L>0$ and, for every $R>0$, there exists $l_{R}>0$ such that

$$
l_{R} \leq \hat{\mathcal{L}}_{\phi}^{n} \mathbb{1}(w) \leq L
$$

for all $n \geq 1$ and all $w \in \mathcal{J}(f) \cap D(0, R)$.

Before going to proof this, let us clarify the situation about the topological pressure.

Corollary 5.18. The $\operatorname{limit}_{\lim } \rightarrow \infty \frac{1}{n} \log \mathcal{L}_{\phi}^{n} \mathbb{1}(w), w \in \mathcal{J}(f)$, exists.

Proof. We start with the proof of Proposition 5.17 by establishing the right hand inequality. Because of Lemma 5.16 we can fix $R_{0}>0$ sufficiently large in order to have $\hat{\mathcal{L}}_{\phi} \mathbb{1}(w) \leq 1$ for all $|w| \geq R_{0}$. We show now by induction that

$$
\left\|\hat{\mathcal{L}}_{\phi}^{n} \mathbb{1}\right\|_{\infty} \leq L:=\frac{K_{\phi, R_{0}}}{m_{\phi}\left(D\left(0, R_{0}\right)\right)} \quad \text { for every } n \geq 0 \text {. }
$$

Here and later in this proof $K_{\phi, R} \geq 1$ is the constant coming from Lemma [5.7 with $\mathcal{L}_{\phi}$ replaced by the normalized operator $\hat{\mathcal{L}}_{\phi}$. For $n=0$ this estimate is immediate. So, suppose that it holds for some $n \geq 0$. Still because of Lemma 5.16, there exists $w_{n+1} \in \mathcal{J}(f)$ such that

$$
\hat{\mathcal{L}}_{\phi}^{n+1} \mathbb{1}\left(w_{n+1}\right)=\left\|\hat{\mathcal{L}}_{\phi}^{n+1} \mathbb{1}\right\|_{\infty}
$$

If $\left|w_{n+1}\right| \geq R_{0}$, then

$$
\left\|\hat{\mathcal{L}}_{\phi}^{n+1} \mathbb{1}\right\|_{\infty}=\hat{\mathcal{L}}_{\phi}^{n+1} \mathbb{1}\left(w_{n+1}\right) \leq\left\|\hat{\mathcal{L}}_{\phi}^{n} \mathbb{1}\right\|_{\infty} \hat{\mathcal{L}}_{\phi} \mathbb{1}\left(w_{n+1}\right) \leq L .
$$

In the other case, $\left|w_{n+1}\right|<R_{0}$, it follows from Lemma 5.7 that

$$
1=\int \hat{\mathcal{L}}_{\phi}^{n+1} \mathbb{1} d m_{\phi} \geq \int_{D\left(0, R_{0}\right)} \hat{\mathcal{L}}_{\phi}^{n+1} \mathbb{1} d m_{\phi} \geq K_{\phi, R_{0}}^{-1} \hat{\mathcal{L}}_{\phi}^{n+1} \mathbb{1}\left(w_{n+1}\right) m_{\phi}\left(D\left(0, R_{0}\right)\right)
$$


and so (5.6) holds. Increasing $R_{0}$ if necessary, we may suppose now that $m_{\phi}(\{|w|>$ $\left.\left.R_{0}\right\}\right) \leq \frac{1}{4 L}$. Let $R>R_{0}$. We have

$$
1=\int \hat{\mathcal{L}}_{\phi}^{n} \mathbb{1} d m_{\phi} \leq \int_{D\left(0, R_{0}\right)} \hat{\mathcal{L}}_{\phi}^{n} \mathbb{1} d m_{\phi}+\frac{1}{4}
$$

Hence, for any $n \geq 0$ there is $z_{n} \in D\left(0, R_{0}\right) \cap \mathcal{J}(f)$ with $\hat{\mathcal{L}}_{\phi}^{n} \mathbb{1}\left(z_{n}\right) \geq 3 / 4$. If $w \in D(0, R) \cap \mathcal{J}(f)$ is any other point we have for any $n \geq 0$

$$
K_{\phi, R} \hat{\mathcal{L}}_{\phi}^{n} \mathbb{1}(w) \geq \hat{\mathcal{L}}_{\phi}^{n} \mathbb{1}\left(z_{n}\right) \geq 3 / 4
$$

which shows the left hand inequality.

The Perron-Frobenius operator $\hat{\mathcal{L}}_{\phi}$ sends the Radon-Nikodym derivatives of Borel probability $f$-invariant measures $\mu$ absolutely continuous with respect to the conformal measures $m_{\phi}$ to the Radon-Nikodym derivatives of measures $\mu \circ f^{-1}$. Hence the positive fixed points of $m_{\phi}$ measure 1 of this Perron-Frobenius operator are in one-to-one correspondence with $f$-invariant measures absolutely continuous with respect to the measures $m_{\phi}$. Therefore, we can now continue in the usual way, namely, use the uniform estimates of the normalized transfer operator given in Proposition 5.17 to construct a fixed point $\rho_{\phi}: \mathcal{J}(f) \rightarrow \mathbb{R}$ of $\hat{\mathcal{L}}_{\phi}$ which then gives the Gibbs (or equilibrium) state $\mu_{\phi}=\rho_{\phi} m_{\phi}$.

Theorem 5.19. There exists a $f$-invariant measure $\mu_{\phi}$ which is absolutely continuous with respect to the conformal measure $m_{\phi}$. Moreover, the density function $\rho_{\phi}=d \mu_{\phi} / d m_{\phi}$ is continuous and satisfies

$$
l_{R} \leq \rho_{\phi}(w) \leq L \quad \text { for every } w \in \mathcal{J}(f) \cap D(0, R)
$$

with $l_{R}, L$ the constants from Proposition 5.17, In addition

$$
\rho_{\phi}(w) \preceq|w|^{-t\left(\underline{\alpha}_{2}-\tau\right)}, w \in \mathcal{J}(f),
$$

hence

$$
\lim _{w \rightarrow \infty} \rho_{\phi}(w)=0
$$

Proof. We have to construct a normalized fixed point $\rho_{\phi}$ of $\hat{\mathcal{L}}_{\phi}$. The natural candidate is a limit of a subsequence of

$$
h_{n}(w):=\frac{1}{n} \sum_{k=1}^{n} \hat{\mathcal{L}}_{\phi}^{k} \mathbb{1}(w) \quad, \quad w \in \mathcal{J}(f) .
$$

For $w_{1}, w_{2} \in \mathcal{J}(f)$ with $\left|w_{1}-w_{2}\right|<\delta$ we have

$$
\left|\hat{\mathcal{L}}_{\phi}^{n} \mathbb{1}(x)-\hat{\mathcal{L}}_{\phi}^{n} \mathbb{1}(y)\right| \preceq \hat{\mathcal{L}}_{\phi}^{n} \mathbb{1}(y)|y-x|^{\beta} \leq L|y-x|^{\beta}
$$

because of Lemma 5.6 and since we have the uniform bound of the normalized transfer operator given in Proposition 5.17. Therefore the sequence $\left(\hat{\mathcal{L}}_{\phi}^{n} \mathbb{1}\right)_{n \geq 1}$ is equicontinuous and the same is true for $\left(h_{n}=\frac{1}{n} \sum_{k=1}^{n} \hat{\mathcal{L}}_{\phi}^{n} \mathbb{1}\right)_{n \geq 1}$. Arzela-Ascoli's Theorem applies: there is a (in fact Hölder-) continuous function $\rho_{\phi}$ such that, for some subsequence $n_{j} \rightarrow \infty, h_{n_{j}} \rightarrow \rho_{\phi}$ uniformly on compact subsets of $\mathcal{J}(f)$. In addition, $\rho_{\phi}$ satisfies (5.8) since all the $h_{n}$ have this property (Proposition 5.17). 
The measure $\mu_{\phi}=\rho_{\phi} m_{\phi}$ is a invariant measure provided we can check that $\rho_{\phi}$ is a fixed point of $\hat{\mathcal{L}}_{\phi}$. In order to do so it suffices to verify

$$
\hat{\mathcal{L}}_{\phi}\left(\rho_{\phi}\right)=\lim _{j \rightarrow \infty} \hat{\mathcal{L}}_{\phi}\left(h_{n_{j}}\right)
$$

since then $\hat{\mathcal{L}}_{\phi}\left(\rho_{\phi}\right)=\rho_{\phi}$ results from $\hat{\mathcal{L}}_{\phi}\left(h_{n}\right)=h_{n}+\frac{1}{n}\left(\hat{\mathcal{L}}_{\phi}^{n+1} \mathbb{1}-\hat{\mathcal{L}}_{\phi} \mathbb{1}\right)$.

So let $w \in \mathcal{J}(f)$ and let $\varepsilon>0$. The series

$$
\sum_{z \in f^{-1}(w)} e^{\phi(z)-P(\phi)}=\hat{\mathcal{L}}_{\phi} \mathbb{1}(w)
$$

being convergent, there exists $R>0$ such that $\sum_{z \in f^{-1}(w),|z|>R} e^{\phi(z)-P(\phi)}<\varepsilon$. Using (5.8) we get

$$
\left|\sum_{z \in f^{-1}(w),|z|>R}\left(h_{n_{j}}(z)-\rho_{\phi}(z)\right) e^{\phi(z)-P(\phi)}\right| \leq 2 L \varepsilon
$$

and, by uniform convergence on compact sets,

$$
\left|\sum_{z \in f^{-1}(w),|z| \leq R}\left(h_{n_{j}}(z)-\rho_{\phi}(z)\right) e^{\phi(z)-P(\phi)}\right| \leq \varepsilon \hat{\mathcal{L}}_{\phi} \mathbb{1}(w) \leq L \varepsilon
$$

for some $j_{0}(\varepsilon, R)$ and every $j \geq j_{0}(\varepsilon, R)$. The property (5.9) follows.

We finally have to check the behavior of $\rho_{\phi}$ near infinity. The function $\rho_{\phi}$ being a fixed point of $\hat{\mathcal{L}}_{\phi}$ we get from Lemma 5.16

$$
\left|\rho_{\phi}(w)\right|=\left|\hat{\mathcal{L}}_{\phi} \rho_{\phi}(w)\right| \leq\left\|\rho_{\phi}\right\|_{\infty} \hat{\mathcal{L}}_{\phi} \mathbb{1}(w) \rightarrow 0 \text { if } w \rightarrow \infty
$$

with polynomial decay given by (5.5). All in all, $d \mu_{\phi}=\rho_{\phi} d m_{\phi}$ defines a $f$-invariant probability measure having all the required properties.

\subsection{The support and uniqueness of the conformal measure}

Keep $m_{\phi}$ to be the $\phi$-conformal measure constructed in Theorem 5.11. This theorem and Lemma 5.6 lead to the following.

Lemma 5.20. If $f: \mathbb{C} \rightarrow \mathbb{C}$ is a dynamically semi-regular function and $\phi: \mathcal{J}(f) \rightarrow$ $\mathbb{R}$ is a tame potential, then for every $z \in \mathcal{J}(f)$, every $v \in f^{-n}(z)$ and every set $B \subset D(z, \delta)$, we have that

$$
\begin{aligned}
K_{\phi}^{-1} \exp \left(S_{n} \phi\left(f_{v}^{-n}(w)\right)\right. & -\mathrm{P}(\phi) n) m_{\phi}(B) \leq \\
m_{\phi}\left(f_{v}^{-n}(B)\right) & =\int_{B} \exp \left(S_{n} \phi\left(f_{v}^{-n}(w)\right)-\mathrm{P}(\phi) n\right) d m_{\phi}(w) \\
& \leq K_{\phi} \exp \left(S_{n} \phi\left(f_{v}^{-n}(w)\right)-\mathrm{P}(\phi) n\right) m_{\phi}(B),
\end{aligned}
$$

where $K_{\phi}$ comes from Lemma 5.6 .

We shall now prove the following. 
Proposition 5.21. Suppose $\nu$ to be an arbitrary $\rho e^{-\phi}$-conformal measure with some $\rho>0$. There then exists $M>0$ such that for $\nu$-a.e. $x \in \mathcal{J}(f)$

$$
\liminf _{n \rightarrow \infty}\left|f^{n}(x)\right| \leq M
$$

Consequently, $\nu\left(I_{\infty}(f)\right)=0$ or equivalently $\nu\left(J_{r}(f)\right)=1$. In particular, these statements hold for $\nu=m_{\phi}$.

Proof. Let $M>1$. For every $z \in f^{-1}\left(D^{c}(0, M)\right)$ we have by the left-hand side of (3.4) that

$$
\left|f^{\prime}(z)\right|_{\tau} \succeq|z|^{\hat{\tau}}|f(z)|^{\underline{\alpha}_{2}-\tau} \geq M \underline{\underline{\alpha}}_{2}-\tau|z|^{\hat{\tau}}
$$

Therefore,

$$
e^{\phi(z)} \leq e^{\|h\|_{\infty}}\left|f^{\prime}(z)\right|_{\tau}^{-t} \preceq M^{-t\left(\underline{\alpha}_{2}-\tau\right)}|z|^{-t \hat{\tau}} .
$$

Cover now $\mathcal{J}(f)$ with countably many open disks $\left\{D\left(w_{n}, \delta\right)\right\}_{n=0}^{\infty}$ centered at $\mathcal{J}(f)$, and then form the partition $\left\{A_{n}\right\}_{n=0}^{\infty}$ inductively as follows. $A_{0}=D\left(w_{0}, \delta\right)$ and $A_{n+1}=D\left(w_{n+1}, \delta\right) \backslash \bigcup_{j=0}^{n} A_{j}$. Take an arbitrary Borel set $B \subset D^{c}(0, M)$. We then have by the Proposition 3.6 that

$$
\begin{aligned}
\nu\left(f^{-1}(B)\right) & =\nu\left(f^{-1}\left(B \cap \bigcup_{n=0}^{\infty} A_{n}\right)\right)=\sum_{n=0}^{\infty} \nu\left(f^{-1}\left(B \cap A_{n}\right)\right) \\
& =\sum_{n=0}^{\infty} \sum_{z \in f^{-1}\left(w_{n}\right)} \nu\left(f_{z}^{-1}\left(B \cap A_{n}\right)\right) \\
& \preceq \sum_{n=0}^{\infty} \sum_{z \in f^{-1}\left(w_{n}\right)} M^{-t\left(\underline{\alpha}_{2}-\tau\right)} \nu\left(B \cap A_{n}\right)|z|^{-t \hat{\tau}} \\
& \preceq M^{-t\left(\underline{\alpha}_{2}-\tau\right)} \sum_{n=0}^{\infty} \nu\left(B \cap A_{n}\right) \\
& =M^{-t\left(\underline{\alpha}_{2}-\tau\right)} \nu(B) .
\end{aligned}
$$

We showed that there is $c>0$ such that for every $B \subset D^{c}(0, M)$

$$
\nu\left(f^{-1}(B)\right) \leq c M^{-t\left(\underline{\alpha}_{2}-\tau\right)} \nu(B) .
$$

Since $B \cap f^{-1}(B) \cap \ldots \cap f^{-(n-1)}(B) \subset D^{c}(0, M)$, we therefore get for every $n \geq 1$ that

$$
\begin{aligned}
\nu(B & \left.\cap f^{-1}(B) \cap \cdots \cap f^{-n}(B)\right) \leq \nu\left(f^{-1}(B) \cap \cdots \cap f^{-n}(B)\right) \\
& =\nu\left(f^{-1}\left(B \cap f^{-1}(B) \cap \ldots \cap f^{-(n-1)}(B)\right)\right) \\
& \leq c M^{-t\left(\underline{\alpha}_{2}-\tau\right)} \nu\left(B \cap \ldots \cap f^{-(n-1)}(B)\right) .
\end{aligned}
$$

Therefore we obtain by induction that

$$
\nu\left(B \cap f^{-1}(B) \cap \cdots \cap f^{-n}(B)\right) \leq\left(c M^{-t\left(\underline{\alpha}_{2}-\tau\right)}\right)^{n} \nu(B) .
$$

Since $\tau<\underline{\alpha}_{2}$, this implies that for all $M$ large enough

$$
\nu\left(\bigcap_{n=0}^{\infty} f^{-n}\left(D^{c}(0, M)\right)\right)=0
$$

and consequently

$$
\nu\left(\bigcup_{k=0}^{\infty} f^{-k}\left(\bigcap_{n=0}^{\infty} f^{-n}\left(D^{c}(0, M)\right)\right)\right)=0 .
$$


The proof is finished.

Theorem 5.22. The measure $m_{\phi}$ is the unique $\rho e^{-\phi}$-conformal measure and the conformal factor is necessary $\rho=e^{P(\phi)}$. In addition, the measure $m_{\phi}$ is ergodic with respect to each iterate of $f$.

Proof. Fix $j \geq 1$ and Suppose that $\nu$ is a $\rho e^{-\phi}$-conformal measure. The same proof as in the case of the measure $m=m_{\phi}$ shows that $\nu\left(I_{\infty}(f)\right)=0$. Let $J_{r, N}(f)$ be the subset of $J_{r}(f)$ defined as follows: $z \in J_{r, N}(f)$ if and only if the trajectory of $z$ under $f^{j}$ has an accumulation point in $\mathcal{J}(f) \cap D(0, N)$. Obviously, $\bigcup_{N} J_{r, N}(f)=J_{r}(f)$ and by Proposition 5.21 there exists $M>0$ such that $\nu\left(J_{r, M}(f)\right)=m\left(J_{r, M}(f)\right)=1$. Fix $z \in J_{r, N}(f)$. Then there exist $y \in$ $\mathcal{J}(f) \cap D(0, N)$ and an increasing sequence $\left\{n_{k}\right\}_{k=1}^{\infty}$ such that $y=\lim _{k \rightarrow \infty} f^{n_{k}}(z)$. Considering for $k$ large enough the sets $f_{z}^{-n_{k}}(D(y, 2 \delta))$ and $f_{z}^{-n_{k}}(D(y, \delta /(2 K)))$, where $f_{z}^{-n_{k}}$ is the holomorphic inverse branch of $f^{n_{k}}$ defined on $D(y, 4 \delta)$ and sending $f^{n_{k}}(z)$ to $z$, using conformality of measures $m$ and $\nu$ along with the distortion control from Lemma (5.3), as well as Koebe's Distortion Theorem, we easily deduce that

$B_{N}(\nu)^{-1} \rho^{-n_{k}} \exp \left(S_{n_{k}} \phi(z)\right) \leq \nu\left(D\left(z, \frac{\delta}{2}\left|\left(f^{n_{k}}\right)^{\prime}(z)\right|^{-1}\right)\right) \leq B_{N}(\nu) \rho^{-n_{k}} \exp \left(S_{n_{k}} \phi(z)\right)$

for all $k \geq 1$ large enough, where $B_{N}(\nu)$ is some constant depending on $\nu$ and $N$. Let $M$ be fixed as above. Fix now $E$, an arbitrary bounded Borel set contained in $J_{r}(f)$ and let $E^{\prime}=E \cap J_{r, M}(f)$. Since $m$ is regular, for every $x \in E^{\prime}$ there exists a radius $r(x) \in(0, \varepsilon)$ of the form from (5.10) (and the corresponding number $n(x)=n_{k}(x)$ for an appropriate $\left.k\right)$ such that

$$
m\left(\bigcup_{x \in E^{\prime}} D(x, r(x)) \backslash E^{\prime}\right) \leq \varepsilon .
$$

Now, by the Besicovič Covering Theorem (see $[\mathrm{G}]$ ), we can choose a countable subcover $\left\{D\left(x_{i}, r\left(x_{i}\right)\right)\right\}_{i=1}^{\infty}$ with $r\left(x_{i}\right) \leq \varepsilon$ and $n\left(x_{i}\right) \geq \varepsilon^{-1}$, from the cover

$$
\{D(x, r(x))\}_{x \in E^{\prime}}
$$

of $E^{\prime}$, of multiplicity bounded by some constant $C \geq 1$, independent of the cover. Therefore, assuming $e^{\mathrm{P}(\phi)}<\rho$ and using (5.10) along with (5.11), we obtain

$$
\begin{aligned}
\nu(E)=\nu\left(E^{\prime}\right) & \leq \sum_{i=1}^{\infty} \nu\left(D\left(x_{i}, r\left(x_{i}\right)\right)\right) \leq B_{M}(\nu) \sum_{i=1}^{\infty} \rho^{-n\left(x_{i}\right)} \exp \left(S_{n\left(x_{i}\right)} \phi\left(x_{i}\right)\right) \\
& \leq B_{M}(\nu) B_{M}(m) \sum_{i=1}^{\infty} m\left(D\left(x_{i}, r\left(x_{i}\right)\right)\right) \rho^{-n\left(x_{i}\right)} e^{\mathrm{P}(\phi) n\left(x_{i}\right)} \\
& \leq B_{M}(\nu) B_{M}(m) C m\left(\bigcup_{i=1}^{\infty} D\left(x_{i}, r\left(x_{i}\right)\right)\right)\left(e^{\mathrm{P}(\phi)} \rho^{-1}\right)^{n\left(x_{i}\right)} \\
& \leq B_{M}(\nu) B_{M}(m) C m\left(\bigcup_{i=1}^{\infty} D\left(x_{i}, r\left(x_{i}\right)\right)\right)\left(e^{\mathrm{P}(\phi)} \rho^{-1}\right)^{\varepsilon^{-1}} \\
& \leq C B_{M}(\nu) B_{M}(m)\left(e^{\mathrm{P}(\phi)} \rho^{-1}\right)^{\varepsilon^{-1}}\left(\epsilon+m\left(E^{\prime}\right)\right) \\
& =C B_{M}(\nu) B_{M}(m)\left(e^{\mathrm{P}(\phi)} \rho^{-1}\right)^{\varepsilon^{-1}}(\epsilon+m(E)) .
\end{aligned}
$$


Hence letting $\varepsilon \searrow 0$ we obtain $\nu(E)=0$ and consequently $\nu(\mathcal{J}(f))=0$ which is a contradiction. We obtain a similar contradiction assuming that $\rho<e^{\mathrm{P}(\phi)}$ and replacing in (5.12) the roles of $m$ and $\nu$. Thus $\beta=e^{\mathrm{P}(\phi)}$ and letting $\epsilon \searrow 0$ again, we obtain from (5.12) that $\nu(E) \leq C B_{M}(\nu) B_{M}(m) m(E)$. Exchanging $m$ and $\nu$, we obtain $m(E) \leq C B_{M}(\nu) B_{M}(m) \nu(E)$. These two conclusions along with the already mentioned fact that $m\left(J_{r}(f)\right)=\nu\left(J_{r}(f)\right)=1$, imply that the measures $m$ and $\nu$ are equivalent with Radon-Nikodym derivatives bounded away from zero and infinity.

Let us now prove that any $e^{\mathrm{P}(\phi)} e^{-\phi}$-conformal measure $\nu$ is ergodic with respect to $f^{j}$. Indeed, suppose to the contrary that $f^{-j}(G)=G$ for some Borel set $G \subset$ $\mathcal{J}(f)$ with $0<\nu(G)<1$. But then the two conditional measures $\nu_{G}$ and $\nu_{\mathcal{J}(f) \backslash G}$

$$
\nu_{G}(B)=\frac{\nu(B \cap G)}{\nu(G)}, \quad \nu_{\mathcal{J}(f) \backslash G}(B)=\frac{\nu(B \cap \mathcal{J}(f) \backslash G)}{\nu(\mathcal{J}(f) \backslash G)}
$$

would be $e^{j \mathrm{P}(\phi)} e^{-S_{j} \phi}$-conformal for $f^{j}$ and mutually singular. This contradiction finishes the proof. 


\section{CHAPTER 6}

\section{Finer properties of Gibbs States}

Finer ergodic and stochastic properties of the Gibbs states can only be obtained if we consider the action of the transfer operator on smoother functions then $C_{b}(\mathcal{J}(f))$. Hölder continuous functions turn out to be fine. The starting point is the two norm inequality of Lemma 6.2 which along with Lemma 6.3 enables us to apply the powerful Ionescu-Tulcea and Marinescu theorem. Its consequence in turn is the so called spectral gap (Theorem 6.5). It tells us that all the eigenvalues of $\hat{\mathcal{L}}_{\phi}$ acting on Hölder functions are in a disk of radius strictly less then one excepted the number 1 which turns out to be a simple eigenvalue with eigenfunction the density $\rho_{\phi}=d \mu_{\phi} / d m_{\phi}$. We describe then how this leads to further ergodic properties of the Gibbs states and also to the Central Limit Theorem via exponential decay of correlations and Liverani-Gordon's method. The studies of Gibbs states are completed by establishing the variational principle and the characterization of tame potentials giving rise to the same Gibbs (equilibrium) states. The latter is done by means of cohomologies.

Throughout this chapter $f: \mathbb{C} \rightarrow \hat{\mathbb{C}}$ is always assumed to be a dynamically semi-regular function.

\subsection{The two norm inequality and the spectral gap}

We first formulate the Ionescu-Tulcea and Marinescu theorem in its full generality, then we verify its hypothesis in our context, and then we derive its consequences, also in our particular dynamical setting.

Theorem 6.1. (Ionescu-Tulcea and Marinescu) Let $(F,|\cdot|)$ be a Banach space equipped with a norm $|\cdot|$ and let $E \subset F$ be its linear subspace. The linear space $E$ is assumed to be endowd with a norm $\|\cdot\|$ which satisfies the following two conditions.

(1) Any bounded subset of the Banach space $E$, with the norm $\|\cdot\|$, is relatively compact as a subset of the Banach space $F$ with the norm $|\cdot|$.

(2) If $\left\{x_{n}: n=1,2, \ldots\right\}$ is a sequence of points in $E$ such that $\left\|x_{n}\right\| \leq K_{1}$ for all $n \geq 1$ and some constant $K_{1}$, and if $\lim _{n \rightarrow \infty}\left|x_{n}-x\right|=0$ for some $x \in F$, then $x \in E$ and $\|x\| \leq K_{1}$.

Let $Q: F \rightarrow F$ be a bounded linear operator which preserves $E$, whose restriction to $E$ is also bounded with respect to the norm $\|\cdot\|$, and which satisfies the following two conditions.

(3) There exists a constant $K$ such that $\left|Q^{n}\right| \leq K$ for all $n=1,2, \ldots$.

(4) $\exists N \geq 1 \quad \exists \tau<1 \quad \exists K_{2}>0 \quad\left\|Q^{N}(x)\right\| \leq \tau\|x\|+K_{2}|x|$ for all $x \in E$.

Then 
(5) There exists at most finitely many eigenvalues of $Q: F \rightarrow F$ of modulus 1 , say $\gamma_{1}, \ldots, \gamma_{p}$.

(6) Let $F_{i}=\left\{x \in F: Q(x)=\gamma_{i} x\right\}, i=1, \ldots, p$. Then $F_{i} \subset E$ and $\operatorname{dim}\left(F_{i}\right)<$ $\infty$.

(7) The operator $Q: F \rightarrow F$ can be represented as

$$
Q=\sum_{i=1}^{p} \gamma_{i} Q_{i}+S
$$

where $Q_{i}$ and $S$ are bounded, $Q_{i}(F)=F_{i}$, $\sup _{n \geq 1}\left|S^{n}\right|<\infty$, and

$$
Q_{i}^{2}=Q_{i}, \quad Q_{i} Q_{j}=0(i \neq j), \quad Q_{i} S=S Q_{i}=0
$$

In addition,

(8) $S(E) \subset E$ and $\left.S\right|_{E}$ considered as a linear operator on $(E,\|\cdot\|)$, is bounded and there exist constants $K_{3}>0$ and $0<\tilde{\tau}<1$ such that

$$
\left\|\left.S^{n}\right|_{E}\right\| \leq K_{3} \tilde{\tau}^{n}
$$

for all $n \geq 1$.

The key ingredient in verifying hypothesis of the above theorem is the following.

Lemma 6.2. If $\phi: \mathcal{J}(f) \rightarrow \mathbb{R}$ is a tame potential with a Hölder exponent $\beta>0$ then there exists a constant $c_{1}>0$ such that

$$
\left\|\hat{\mathcal{L}}_{\phi}^{n} g\right\|_{\beta} \leq \frac{1}{2}\|g\|_{\beta}+c_{1}\|g\|_{\infty}
$$

for all $n \geq 1$ large enough and every $g \in \mathrm{H}_{\beta}$. In particular, $\hat{\mathcal{L}}_{\phi}\left(\mathrm{H}_{\beta}\right) \subset \mathrm{H}_{\beta}$.

Proof. Fix $n \geq 1, g \in \mathrm{H}_{\beta}$ and $x, y \in \mathcal{J}(f)$ with $|y-x| \leq \delta$. Put $V_{n}=f^{-1}(x)$ and $\phi_{n}=\exp \left(S_{n} \phi-\mathrm{P}(\phi) n\right)$. Then

$$
\begin{aligned}
\left|\hat{\mathcal{L}}_{\phi}^{n} g(y)-\hat{\mathcal{L}}_{\phi}^{n} g(x)\right| & =\left|\sum_{v \in V_{n}} \phi_{n}\left(f_{v}^{-n}(y)\right) g\left(f_{v}^{-n}(y)\right)-\sum_{v \in V_{n}} \phi_{n}\left(f_{v}^{-n}(x)\right) g\left(f_{v}^{-n}(x)\right)\right| \\
& \leq \sum_{v \in V_{n}}\left|g\left(f_{v}^{-n}(y)\right)\right|\left|\phi_{n}\left(\left(f_{v}^{-n}\right)(y)\right)-\phi_{n}\left(\left(f_{v}^{-n}\right)(x)\right)\right|+ \\
& +\sum_{v \in V_{n}} \phi_{n}\left(\left(f_{v}^{-n}\right)(x)\right)\left|g\left(f_{v}^{-n}(y)-g\left(f_{v}^{-n}(x)\right)\right)\right| .
\end{aligned}
$$

This can be estimated by using Lemma 5.3 for the first term, Koebe's distortion theorem (Lemma 4.6) together with the expanding property for the second term 
and by employing Proposition 5.17 as follows:

$$
\begin{aligned}
\mid \hat{\mathcal{L}}_{\phi}^{n} g(y) & -\hat{\mathcal{L}}_{\phi}^{n} g(x) \mid \preceq \\
& \preceq \sum_{v \in V_{n}}\|g\|_{\infty} \sum_{v \in V_{n}} \phi_{n}\left(f_{v}^{-n}(x)\right) \cdot|x-y|^{\beta} \\
& +\sum_{v \in V_{n}}\left|\phi_{n}\left(f_{v}^{-n}\right)(x)\right| v_{\beta}(g)\left|f_{v}^{-n}(y)-f_{v}^{-n}(x)\right|^{\beta} \\
& \leq\|g\|_{\infty} \hat{\mathcal{L}}_{\phi}^{n} \mathbb{1}(x)|y-x|^{\beta}+v_{\beta}(g)\left(c \gamma^{-n}\right)^{\beta}|y-x|^{\beta} \sum_{v \in V_{n}}\left|\phi_{n}\left(f_{v}^{-n}(x)\right)\right| \\
& \leq L\left(\|g\|_{\infty}+c^{\beta} \gamma^{-\beta n} v_{\beta}(g)\right)|y-x|^{\beta} .
\end{aligned}
$$

This shows that there are $c_{1}, c_{2}>0$ such that

$$
v_{\beta}\left(\hat{\mathcal{L}}_{\phi}^{n} g\right) \leq c_{1}\|g\|_{\infty}+c_{2} \gamma^{-\beta n}\|g\|_{\beta}<\infty .
$$

In particular $\hat{\mathcal{L}}_{\phi}^{n}(g) \in \mathrm{H}_{\beta}$. The inclusion $\hat{\mathcal{L}}_{\phi}\left(H_{\beta}\right) \subset H_{\beta}$ is proved. It then follows from (6.2) that

$$
\begin{aligned}
\left\|\hat{\mathcal{L}}_{\phi}^{n} g\right\|_{\beta} & \leq c_{2} \gamma^{-\beta n}\|g\|_{\beta}+c_{1}\|g\|_{\infty}+\left\|\hat{\mathcal{L}}_{\phi}^{n} g\right\|_{\infty} \\
& \leq c_{2} \gamma^{-\beta n}\|g\|_{\beta}+\left(c_{1}+L\right)\|g\|_{\infty} .
\end{aligned}
$$

The proof is thus finished by taking $n \geq 1$ so large that $c_{2} \gamma^{-\beta n} \leq \frac{1}{2}$.

In order to apply the theorem of Ionescu-Tulcea and Marinescu we need the following.

Lemma 6.3. Suppose that $\phi: \mathcal{J}(f) \rightarrow \mathbb{R}$ is a tame potential. If $B$ is a bounded subset of $\mathrm{H}_{\beta}$ (with the $\|\cdot\|_{\beta}$ norm), then $\hat{\mathcal{L}}_{\phi}(B)$ is a pre-compact subset of $C_{b}$ (with the $\|\cdot\|_{\infty}$ norm).

Proof. Fix an arbitrary sequence $\left\{g_{n}\right\}_{n=1}^{\infty} \subset B$. Since by (6.1) the family $\hat{\mathcal{L}}_{\phi}(B)$ is equicontinuous and, since the operator $\hat{\mathcal{L}}_{\phi}$ is bounded, this family is bounded, it follows from Ascoli's theorem that we can choose from $\left\{\hat{\mathcal{L}}_{\phi}\left(g_{n}\right)\right\}_{n=1}^{\infty}$ an infinite subsequence $\left\{\hat{\mathcal{L}}_{\phi}\left(g_{n_{j}}\right)\right\}_{j=1}^{\infty}$ converging uniformly on compact subsets of $\mathcal{J}(f)$ to a function $\psi \in C_{b}$. Fix now $\varepsilon>0$. Since $B$ is a bounded subset of $C_{b}$, it follows from Lemma $\left[5.16\right.$ that there exists $R>0$ such that $\left|\hat{\mathcal{L}}_{\phi} g(z)\right| \leq \varepsilon / 2$ for all $g \in B$ and all $z \in \mathcal{J}(f) \cap D^{c}(O, R)$. Hence

$$
|\psi(z)| \leq \varepsilon / 2
$$

for all $z \in \mathcal{J}(f) \cap D^{c}(O, R)$. Thus $\left|\hat{\mathcal{L}}_{\phi}\left(g_{n_{j}}\right)(z)-\psi(z)\right| \leq \varepsilon$ for all $j \geq 1$ and all $z \in$ $\mathcal{J}(f) \cap D^{c}(O, R)$. In addition, there exists $p \geq 1$ such that $\left|\hat{\mathcal{L}}_{\phi}\left(g_{n_{j}}\right)(z)-\psi(z)\right| \leq \varepsilon$ for every $j \geq p$ and every $z \in \mathcal{J}(f) \cap D(0, R)$. Therefore $\left|\hat{\mathcal{L}}_{\phi}\left(g_{n_{j}}\right)(z)-\psi(z)\right| \leq \varepsilon$ for all $j \geq p$ and all $z \in \mathcal{J}(f)$. This means that $\left\|\hat{\mathcal{L}}_{\phi}\left(g_{n_{j}}\right)-\psi\right\|_{\infty} \leq \varepsilon$ for all $j \geq p$. Letting $\varepsilon \searrow 0$ we conclude from this and from (6.3) that $\hat{\mathcal{L}}_{\phi}\left(g_{n_{j}}\right)$ converges uniformly on $\mathcal{J}(f)$ to $\psi \in C_{b}$. We are done.

Combining now Lemma 6.2 and Lemma 6.3. we see that the assumptions of Theorem 1.5 in IM are satisfied with Banach spaces $\mathrm{H}_{\beta} \subset C_{b}$ and the bounded operator $\hat{\mathcal{L}}_{\phi}: C_{b} \rightarrow C_{b}$ preserves $\mathrm{H}_{\beta}$. It gives us the following, where the fact that 
the unitary eigenvalues form a cyclic group follows from Lemma 18, Theorem 4.9 and Exercise 2 (p. 326/327) in [Sch].

Theorem 6.4. If $\phi: \mathcal{J}(f) \rightarrow(0, \infty)$ is a tame potential with a Hölder exponent $\beta$, then there exist a finite cyclic group $\gamma_{1}, \ldots, \gamma_{p} \in S^{1}=\{z \in \mathbb{C}:|z|=1\}$, finitely many bounded finitely dimensional operators $Q_{1}, \ldots, Q_{p}: \mathrm{H}_{\beta} \rightarrow \mathrm{H}_{\beta}$ and an operator $S: \mathrm{H}_{\beta} \rightarrow \mathrm{H}_{\beta}$ such that

$$
\hat{\mathcal{L}}_{\phi}^{n}=\sum_{i=1}^{p} \gamma_{i}^{n} Q_{i}+S^{n}
$$

for all $n \geq 1$,

$$
Q_{i}^{2}=Q_{i}, Q_{i} \circ Q_{j}=0,(i \neq j), Q_{i} \circ S=S \circ Q_{i}=0
$$

and

$$
\left\|S^{n}\right\|_{\beta} \leq C \xi^{n}
$$

for some constant $C>0$, some constant $\xi \in(0,1)$ and all $n \geq 1$. In particular all numbers $\gamma_{1}, \ldots, \gamma_{p}$ are isolated eigenvalues of the operator $\hat{\mathcal{L}}_{\phi}: \mathrm{H}_{\beta} \rightarrow \mathrm{H}_{\beta}$ and this operator is quasi-compact.

We can now prove the following culminating result of this section concerning the spectrum of $\hat{\mathcal{L}}_{\phi}$ acting on the Hölder space $\mathrm{H}_{\beta}$ with $\beta$ the exponent of the potential $\phi$. Note that we already know an eigenfunction of the eigenvalue 1 which is the density $\rho_{\phi}=d \mu_{\phi} / d m_{\phi}$. This function is a fixed point of $\hat{\mathcal{L}}_{\phi}$ because it has been constructed as a limit $\rho_{\phi}=\lim _{j \rightarrow \infty} \frac{1}{n_{j}} \sum_{k=1}^{n_{j}} \hat{\mathcal{L}}_{\phi}^{k} \mathbb{1}$ (cf. Proposition 5.19). It follows from Lemma 6.2 that $\rho_{\phi}$ is in $\mathrm{H}_{\beta}$.

Theorem 6.5. Let $\phi: \mathcal{J}(f) \rightarrow(0, \infty)$ be a tame potential with a Hölder exponent $\beta$. Then we have the following.

(a) The number 1 is a simple isolated eigenvalue of the operator $\hat{\mathcal{L}}_{\phi}: \mathrm{H}_{\beta} \rightarrow \mathrm{H}_{\beta}$ and all other eigenvalues are contained in a disk of radius strictly smaller than 1.

(b) With $S: \mathrm{H}_{\beta} \rightarrow \mathrm{H}_{\beta}$ as in Theorem 6.4, we have

$$
\hat{\mathcal{L}}_{\phi}=Q_{1}+S,
$$

where $Q_{1}: \mathrm{H}_{\beta} \rightarrow \mathbb{C} \rho_{\phi}$ is a projector on the eigenspace $\mathbb{C} \rho_{\phi}$ (given by the formula $Q_{1}(g)=\left(\int g d m_{\phi}\right) \rho_{\phi}, Q_{1} \circ S=S \circ Q_{1}=0$ and

$$
\left\|S^{n}\right\|_{\beta} \leq C \xi^{n}
$$

for some constant $C>0$, some constant $\xi \in(0,1)$ and all $n \geq 1$.

Here is a useful application. As we explain in a while, this property yields in particular mixing of the system (and is sometimes called directly mixing).

Corollary 6.6. With the notations of Theorem 6.5 we have, for every $n \geq 1$, that $\hat{\mathcal{L}}_{\phi}^{n}=Q_{1}+S^{n}$ and that $\hat{\mathcal{L}}_{\phi}^{n}(g) \rightarrow\left(\int g d m_{\phi}\right) \rho_{\phi}$ exponentially when $n \rightarrow \infty$. More precisely,

$$
\left\|\hat{\mathcal{L}}_{\phi}^{n}(g)-\left(\int g d m_{\phi}\right) \rho_{\phi}\right\|_{\beta}=\left\|S^{n}(g)\right\|_{\beta} \leq C \xi^{n}\|g\|_{\beta} \quad, g \in H_{\beta} .
$$


Proof. We first show that 1 is the only unitary eigenvalue and that it is a simple eigenvalue which means that the associated eigenspace is generated by the density $\rho_{\phi}$. So, suppose that

$$
\hat{\mathcal{L}}_{\phi} g=\xi g
$$

with some $\xi \in \mathbb{C}$ of modulus one and some non-zero $g \in H_{\beta}$. Since, by Theorem 6.4 the unitary eigenvalues form a finite cyclic group, there exists $l \geq 1$ such that $\xi^{l}=1$. We then have

$$
\hat{\mathcal{L}}_{\phi}^{l} g=g .
$$

Since $\hat{\mathcal{L}}_{\phi}$ preserves the class of real-valued functions, the same is true for $\operatorname{Re} g$ and also for Im $g$. Hence, it is sufficient to consider real such $g: \mathcal{J}(f) \rightarrow \mathbb{R}$. Denote $g_{0}^{+}=\max \{0, g\}$ and $g_{0}^{-}=\min \{0, g\}$. The operator $\hat{\mathcal{L}}_{\phi}$ being positive, we have $g_{1}^{+}=\hat{\mathcal{L}}_{\phi}^{l} g_{0}^{+} \geq 0, g_{1}^{-}=\hat{\mathcal{L}}_{\phi}^{l} g_{0}^{-} \leq 0$ and $g=\hat{\mathcal{L}}_{\phi}^{l} g=g_{1}^{+}+g_{1}^{-}$. Clearly there is not a unique decomposition of $g$ in a positive and a negative function. But The functions $g_{0}^{+}, g_{0}^{-}$are extremal in the sense that they are the smallest functions that have this property. Consequently $g_{1}^{+} \geq g_{0}^{+}$and $g_{1}^{-} \leq g_{0}^{-}$. Since these functions are continuous and since $\int g_{1}^{+} d m_{\phi}=\int g_{0}^{+} d m_{\phi}$ we have $g_{1}^{+}=g_{0}^{+}$and, for the same reasons, $g_{1}^{-} \leq g_{0}^{-}$.

One of these two functions is not identically zero. Suppose that $g_{0}^{+}$has this property. Then $\tilde{\mu}=g_{0}^{+} m_{\phi}$ is a positive measure of finite mass that is $f^{l}$-invariant and equivalent to $\mu_{\phi}=\rho_{\phi} m_{\phi}$. Since, by Theorem 5.22, the measure $\mu_{\phi}$ is ergodic with respect to $f^{l}$, we conclude that $g_{0}^{+} m_{\phi}=c \rho_{\phi} m_{\phi}$ for some $c>0$. Consequently $g_{0}^{+}=c \rho_{\phi} \in \mathbb{C} \rho_{\phi}$. The same argument is valid for the negative parts $g_{0}^{-}$provided it is not identically zero. It does follow that the initial function $g \in \mathbb{C} \rho_{\phi}$ and that 1 is the only unitary eigenvalue.

The spectral gap comes now from Theorem 6.4. It remains to justify the claimed form of the projector $Q_{1}$. If $Q_{1}(\psi)=k \rho_{\phi}$, then

$$
k=\int k \rho_{\phi} d m_{\phi}=\int Q_{1}^{n} \psi d m_{\phi} \text { for every } n \geq 1 .
$$

Along with the equality $\hat{\mathcal{L}}_{\phi}^{n}=Q_{1}^{n}+S^{n}$ and the formula $\left\|S^{n}\right\|_{\alpha} \leq C \xi^{n}$ the claim follows.

\subsection{Ergodic properties of Gibbs States}

We now investigate further the Gibbs state $\mu_{\phi}$ of the potential $\phi$ (cf. Theroem 5.15). Due to Theorem 6.5 this $f$-invariant measure $\mu_{\phi}$ has much finer stochastic properties than ergodicity of all iterates of $f$. These follow after the following definitions.

Definition 6.7. The set

$$
\mathcal{J}(f)=\left\{\left\{\omega_{n}\right\}_{n=0}^{\infty} \in \mathcal{J}(f)^{\infty}: f\left(w_{n+1}\right)=w_{n} \text { for all } n \geq 0\right\}
$$

is called the Rokhlin natural extension of $\mathcal{J}(f)$. Notice that the map $\tilde{f}: \mathcal{J}(f) \rightarrow$ $\mathcal{J}(f)$ given by the formula

$$
\tilde{f}\left(\left\{\omega_{n}\right\}_{n=0}^{\infty}\right)=\left\{f\left(\omega_{n}\right)\right\}_{n=0}^{\infty}
$$


is a homeomorphism. For every $n \geq 0$ let $\pi_{n}: \mathcal{J}(f) \rightarrow \mathcal{J}(f)$ be the projection given by the formula

$$
\pi_{n}\left(\left\{\omega_{n}\right\}_{n=0}^{\infty}\right)=\omega_{n} .
$$

It is well-known that for every Borel probability $f$-invariant measure on $\mathcal{J}(f)$ there exists a unique Borel probability $\tilde{f}$-invariant measure $\tilde{\mu}$ on $\mathcal{J}(f)$ such that $\tilde{\mu} \circ \pi_{n}^{-1}=$ $\mu$ for all $n \geq 0$. The dynamical system $(\mathcal{J}(f), \tilde{f}: \mathcal{J}(f) \rightarrow \mathcal{J}(f), \tilde{\mu})$ is called the Rokhlin natural extension of the dynamical system $(\mathcal{J}(f), f: \mathcal{J}(f) \rightarrow \mathcal{J}(f), \mu)$.

Definition 6.8. A measure preserving authomorphism $(X, \mathcal{A}, T: X \rightarrow X, \mu)(\mathcal{A}$ is the $\sigma$-algebra on $X$ with respect to which the map $T: X \rightarrow X$ is measurable) is said to be $K$-mixing if for an arbitrary finite collection $A_{0}, A_{1}, A_{2}, \ldots A_{r}$ of subsets from $\mathcal{A}$, we have

$$
\lim _{n \rightarrow \infty} \sup \left\{\left|\mu\left(A_{0} \cap B\right)-\mu\left(A_{0}\right) \mu(B)\right|\right\}=0,
$$

where, for every $n \geq 1$, the supremum is taken over all sets $B$ from the sub $\sigma$-algebra of $\mathcal{A}$ generated by the sets $\left\{T^{j}\left(A_{i}\right): 1 \leq i \leq r, j \geq n\right\}$.

$K$-mixing is a very strong stochastic property. Any $K$-mixing authomorphism is ergodic, and moreover, it is mixing of any order. The corresponding concept to $K$-mixing for non-invertible maps is that of metric exactnes.

Definition 6.9. A measure preserving endomorphism $(X, \mathcal{A}, T: X \rightarrow X, \mu)$ is metrically exact provided that the $\sigma$-algebra $\bigcap_{n=0}^{\infty} T^{-n}(\mathcal{A})$ is trivial, i.e. consists only of sets of measure 0 and 1.

The link between the concepts recalled in the three above definitions is given by the following (see for instance [KFS or [PU]).

Theorem 6.10. If a measure preserving endomorphism $(X, \mathcal{A}, T: X \rightarrow X, \mu)$ is metrically exact, then its Rokhlin natural extension $(\tilde{T}, \tilde{\mu})$ is $K$-mixing.

We shall now prove the following.

Theorem 6.11. The dynamical system $\left(f: \mathcal{J}(f) \rightarrow \mathcal{J}(f), \mu_{\phi}\right)$ is metrically exact, and consequently, its Rokhlin natural extension is K-mixing.

Proof. Put $\mu=\mu_{\phi}, m=m_{\phi}$ and $\rho=\rho_{\phi}$. Denote by $\mathcal{B}$ the Borel $\sigma$-algebra of $\mathcal{J}(f)$. According to Definition 6.9 we are to show $\bigcap_{n=0}^{\infty} T^{-n}(\mathcal{B})$ consists only of sets of measure 0 or 1 . In order to prove this property, let $A \in \bigcap_{n=0}^{\infty} T^{-n}(\mathcal{B})$ and $\mu(A)>0$. Then for any $n \geq 0$ there exists a set $A_{n} \in \mathcal{B}$ such that $A=f^{-n}\left(A_{n}\right)$. Hence for any $\psi \in C_{b}$ and $n \geq 0$ it follows that

$$
\int_{A} \psi d m=\int_{A_{n}} \hat{\mathcal{L}}_{\phi}^{n}(\psi) d m
$$


and

$$
\int_{A_{n}}\left(\int \psi d m\right) \rho d m=\int_{A_{n}}\left(\int \psi d m\right) d \mu=\mu\left(A_{n}\right) \int \psi d m=\mu(A) \int \psi d m .
$$

Fix now $\varepsilon>0$. By Corollary 6.6 there exists $N \geq 1$ so large that $\| \hat{\mathcal{L}}_{\phi}^{N}(\psi)-$ $\left(\int \psi d m\right) h \| \leq \epsilon$. Therefore, using (6.4) and (6.5), we obtain

$$
\begin{aligned}
\left|\int_{A} \psi d m-\mu(A) \int \psi d m\right| & =\left|\int_{A_{N}}\left(\int \psi d m\right) h d m-\int_{A_{N}} \hat{\mathcal{L}}_{\phi}^{N}(\psi) d m\right| \\
& \leq \int_{A_{N}}\left\|\hat{\mathcal{L}}_{\phi}^{N}(\psi)-\left(\int \psi d m\right) h\right\| d m \\
& \leq \varepsilon m\left(A_{N}\right) \leq \varepsilon
\end{aligned}
$$

and, letting $\varepsilon \rightarrow 0$

$$
\int_{A} \psi d m=\mu(A) \int \psi d m
$$

Setting $\psi=1$ we obtain $m(A)=\mu(A)$ and therefore the formula

$$
\tilde{m}(B)=\frac{\int_{A} \chi_{B} d m}{\mu(A)} \quad(B \in \mathcal{B})
$$

defines a probability measure on the Borel field $\mathcal{B}$. In view of $(\underline{6.6})$ we have that

$$
\int \psi d \tilde{m}=\frac{\int_{A} \psi d m}{\mu(A)}=\int \psi d m
$$

for any $\psi \in C_{b}$. Hence the measures $\tilde{m}$ and $m$ are equal. By (6.7), $m\left(A^{c}\right)=$ $\tilde{m}\left(A^{c}\right)=0$. Therefore $\mu\left(A^{c}\right)=0$ and we are done.

\subsection{Decay of correlations and Central Limit Theorem}

This topic concerns the asymptotic behavior of sums $S_{n} \psi=\sum_{n=0}^{n-1} \psi \circ f^{k}$ for appropriate $\psi: \mathcal{J}(f) \rightarrow \mathbb{R}$. Since the Gibbs state $\mu_{\phi}$ is ergodic it follows from Birkhoffs ergodic Theorem that

$$
\frac{1}{n} S_{n} \psi(z) \longrightarrow \int \psi d \mu_{\phi} \text { for } \mu_{\phi}-\text { a.e. } z \in \mathcal{J}(f) .
$$

In the centered case $\left(\int \psi d \mu_{\phi}=0\right)$, that we consider from now on, it follows in particular that the sequence $\frac{1}{n} S_{n} \psi \rightarrow 0 \mu_{\phi}$-almost surely.

Denote

$$
U(\psi)=\psi \circ f
$$

and consider $X_{k}=U^{k} \psi=\psi \circ f^{k}$. Due to the invariance of $\mu_{\phi}$, these $X_{k}$ are random variables that have all the same distribution. The classical Central Limit Theorem (CLT) says that $\frac{1}{\sqrt{n}} S_{n} \psi$ converges in distribution to a Gaussian random variable $\mathcal{N}\left(0, \sigma^{2}\right)$ if $\sigma^{2}>0$ and, most importantly, if the variables $X_{k}$ are independent. This is however not the case and the defect of independence of $\psi$ and $U^{n} \psi=\psi \circ f^{n}$ is measured by the correlation $C_{n}(\psi, \psi)=C\left(\psi, \psi \circ f^{n}\right)$ defined below. We will see that the mixing property of Corollary 6.6 yields exponential decay of this correlation function. This kind of asymptotic independence for these variables allow to apply Liverani-Gordon's method and to show that the CLT is satisfied. 
We start with two observations concerning the operator $U^{*}$ dual to $U$. The first one says that $U^{*}$ is conjugate to the transfer operator.

Lemma 6.12. The dual operator $U^{*}$ of the restriction of $U$ to $L^{2}\left(\mu_{\phi}\right)$ is given by

$$
U^{*} g=\frac{\hat{\mathcal{L}}_{\phi}\left(g \rho_{\phi}\right)}{\rho_{\phi}} \quad m_{\phi}-\text { a.e. }
$$

for $g \in L^{2}\left(\mu_{\phi}\right)$, where $\rho_{\phi}$ denotes the density $\frac{d \mu_{\phi}}{d m_{\phi}}$ as before.

Proof. If $g, \psi \in L^{2}\left(\mu_{\phi}\right)$, then, still by (5.3),

$$
\begin{aligned}
<U^{*}(g), \psi> & =<g, U(\psi)>=\int g(\psi \circ f) d \mu_{\phi} \\
& =\int g \rho_{\phi}(\psi \circ f) d m_{\phi}=\int \hat{\mathcal{L}}_{\phi}\left(g \rho_{\phi}\right) \psi d m_{\phi} \\
& =\int \frac{\hat{\mathcal{L}}_{\phi}\left(g \rho_{\phi}\right)}{\rho_{\phi}} \psi d \mu_{\phi}=<\frac{\hat{\mathcal{L}}_{\phi}\left(g \rho_{\phi}\right)}{\rho_{\phi}}, \psi>.
\end{aligned}
$$

Lemma 6.13. The operator $U^{k} \circ\left(U^{*}\right)^{k}$ is the orthogonal projection of $L^{2}\left(\mu_{\phi}\right)$ onto $U^{k}\left(L^{2}\left(\mu_{\phi}\right)\right)$ for any $k \geq 0$.

Proof. Let $g \in L^{2}\left(\mu_{\phi}\right)$. We only need to show that for

$$
\tilde{\psi}=\psi \circ f^{k}, \psi \in L^{2}\left(\mu_{\phi}\right),
$$

we have

$$
<g-U^{k} U^{* k} g, \tilde{\psi}>=0 .
$$

But this follows immediately from the $f$-invariance of $\mu_{\phi}$ :

$$
<U^{k} U^{* k} g, U^{k} \psi>=<U^{* k} g, \psi>=<g, U^{k} \psi>\text {. }
$$

6.3.1. Observables. We consider a space of observables which goes beyond bounded Hölder functions because we want that it contains in particular all loosely tame potentials.

Definition 6.14. For $\beta \in(0,1]$ and with $\rho_{\phi}=d \mu_{\phi} / d m_{\phi}$ we set

$$
\mathcal{O}_{\beta}=\left\{\psi: \mathcal{J}(f) \rightarrow \mathbb{C} ; \hat{\mathcal{L}}_{\phi}\left(\rho_{\phi} \psi\right) \in \mathrm{H}_{\beta} \text { and } \psi \in L_{m_{\phi}}^{2}\right\}=\frac{1}{\rho_{\phi}} \hat{\mathcal{L}}_{\phi}^{-1}\left(\mathrm{H}_{\beta}\right) \cap L_{m_{\phi}}^{2} .
$$

Lemma 6.15. If $\psi$ is a loosely tame potential, then $\psi \in \mathcal{O}_{\beta}$.

Proof. This fact is a particular case of Lemma 7.9. So we postpone the proof to Chapter 7. 
6.3.2. Decay of Correlations. Let $\psi_{1}$ and $\psi_{2}$ be real square $\mu_{\phi}$-integrable functions on $\mathcal{J}(f)$. For every positive integer $n$ the $n$-th correlation of the pair $\psi_{1}, \psi_{2}$, is the number

$$
C_{n}\left(\psi_{1}, \psi_{2}\right):=\int \psi_{1} \cdot\left(\psi_{2} \circ f^{n}\right) d \mu_{\phi}-\int \psi_{1} d \mu_{\phi} \int \psi_{2} d \mu_{\phi}
$$

provided the above integrals exist. Notice that, due to the $f$-invariance of $\mu_{\phi}$, we can also write

$$
C_{n}\left(\psi_{1}, \psi_{2}\right)=\int\left(\psi_{1}-E \psi_{1}\right)\left(\left(\psi_{2}-E \psi_{2}\right) \circ f^{n}\right) d \mu_{\phi},
$$

where we put $E \psi=\int \psi d \mu_{\phi}$.

Theorem 6.16. There exists $C \geq 1$ such that for all $\psi_{1} \in \mathcal{O}_{\beta}, \psi_{2} \in L^{1}\left(m_{\phi}\right)$

$$
\left|C_{n}\left(\psi_{1}, \psi_{2}\right)\right| \leq C \xi^{n}\left\|\hat{\mathcal{L}}_{\phi}\left(\left(\psi_{1}-E \psi_{1}\right) \rho_{\phi}\right)\right\|_{\beta}\left\|\psi_{2}-E \psi_{2}\right\|_{L^{1}\left(m_{\phi}\right)},
$$

where $\xi \in(0,1)$ comes from Theorem 6.5(b).

Proof. Replacing $\psi_{i}$ by $\psi_{i}-E\left(\psi_{i}\right)$ if necessary we may suppose that the mean of the $\psi_{i}, i=1,2$, is zero. With (5.3) we have that

$$
C_{n}\left(\psi_{1}, \psi_{2}\right)=\int \psi_{1} \psi_{2} \circ f^{n} \rho_{\phi} d m_{\phi}=\int \hat{\mathcal{L}}_{\phi}^{n}\left(\psi_{1} \rho_{\phi}\right) \psi_{2} d m_{\phi}
$$

But

$$
\left|\hat{\mathcal{L}}_{\phi}^{n}\left(\psi_{1} \rho_{\phi}\right)\right|=\left|S^{n-1}\left(\hat{\mathcal{L}}_{\phi}\left(\psi_{1} \rho_{\phi}\right)\right)\right| \leq c \xi^{n-1}\left\|\hat{\mathcal{L}}_{\phi}\left(\psi_{1} \rho_{\phi}\right)\right\|_{\beta}
$$

because of Corollary 6.6 and since $\hat{\mathcal{L}}_{\phi}\left(\psi_{1} \rho_{\phi}\right) \in \mathrm{H}_{\beta}$. Hence,

$$
\left|C_{n}\left(\psi_{1}, \psi_{2}\right)\right| \preceq \xi^{n}\left\|\hat{\mathcal{L}}_{\phi}\left(\psi_{1} \rho_{\phi}\right)\right\|_{\beta}\left\|\psi_{2}\right\|_{L^{1}\left(m_{\phi}\right)}
$$

as claimed.

6.3.3. The Central Limit Theorem. Our next goal is to prove the Central Limit Theorem (CLT) for a large class of random variables induced by the dynamical system $f: \mathcal{J}(f) \rightarrow \mathcal{J}(f)$ via Gordin-Liverani's method. The variables under considerations are $X_{k}=U^{k} \psi=\psi \circ f^{k}$. Let us recall that CLT means that $\frac{1}{\sqrt{n}} S_{n} \psi=\frac{1}{\sqrt{n}} \sum_{k=0}^{n-1} U^{k} \psi(z)$ converges in distribution to the Gaussian random variable $\mathcal{N}\left(0, \sigma^{2}\right)$. More precisely, for any $t \in \mathbb{R}$,

$$
\mu\left(\left\{z \in \mathcal{J}(f): \frac{1}{\sqrt{n}} S_{n} \psi(z) \leq t\right\}\right) \rightarrow \frac{1}{\sigma \sqrt{2 \pi}} \int_{-\infty}^{t} \exp \left[-u^{2} / 2 \sigma^{2}\right] d u .
$$

Theorem 6.17. If $\psi$ is any loosely tame $\beta$-Hölder continuous function or, more generally, if $\psi \in \bigcup_{\beta \in(0,1]} \mathcal{O}_{\beta}$, then the asymptotic variance

$$
\sigma^{2}=\sigma_{\mu_{\phi}}^{2}(\psi) \leq \hat{\sigma}_{\mu_{\phi}}^{2}(\psi)=\int \psi^{2} d \mu_{\phi}+2 \sum_{k=1}^{\infty} \int \psi \psi \circ f^{k} d \mu_{\phi}
$$

exists and one of the following two cases occurs:

(i) If $\sigma^{2}>0$, then the CLT holds.

(ii) If $\sigma^{2}=0$, then $U \psi=\psi \circ f$ is measurably cohomologous to zero. 
In addition, if $\psi$ is a bounded function, then equality in (6.10) holds.

Case (ii) is usually the exceptional situation. We come back to this in the next section.

The rest of this section is devoted to the proof of CLT. A way of establishing it under very weak independence assumptions is to use Martingales approximations. This method was initialized by Gordin Go and then used by several other authors including Liverani $[\mathbf{L i v}$. The method by Gordin works under the condition

$L^{2}$-convergence: $\quad \sum_{k=0}^{\infty}\left\|U^{k} U^{* k} \psi\right\|_{L^{2}\left(\mu_{\phi}\right)}<\infty$.

Liverani used the following weaker condition.

$L^{1}$-convergence:

$$
\begin{aligned}
& \sum_{k=0}^{\infty}\left|\int \psi \psi \circ f^{k} d \mu_{\phi}\right|<\infty \text { and } \\
& \sum_{k=0}^{\infty} U^{* k} \psi \text { converges in } L^{1}\left(\mu_{\phi}\right) .
\end{aligned}
$$

Note however that he makes in addition the assumption $\psi \in L^{\infty}$ which is not the case for functions of $\mathcal{O}_{\beta}$. We will see that we are somehow in an intermediate situation.

Notice also that the first sum of the above condition involves $\operatorname{Cor}\left(\psi, \psi \circ f^{k}\right)=$ $\int \psi \psi \circ f^{k} d \mu_{\phi}$ (we still suppose $\int \psi d \mu_{\phi}=0$ ) which explains that this is in fact a weak asymptotic independence condition for the variables $\psi \circ f^{k}$.

Lemma 6.18. If $\psi \in \mathcal{O}_{\beta}$ is bounded with $\int \psi d \mu_{\phi}=0$ then the $L^{2}$-convergence property holds. For general mean zero $\psi \in \mathcal{O}_{\beta}$ the $L^{1}$-convergence property is satisfied and, moreover,

$$
\left\|U^{* k} \psi\right\|_{L^{1}\left(\mu_{\phi}\right)} \preceq \xi^{k}
$$

for any $k \geq 1$ where $\xi \in(0,1)$.

Proof. Consider first $\psi \in \mathcal{O}_{\beta}$ with $\int \psi d \mu_{\phi}=0$. From the relation between $U^{*}$ and the transfer operator given in Lemma 6.12 we have $U^{* k} \psi=\frac{1}{\rho_{\phi}} \hat{\mathcal{L}}_{\phi}^{k}\left(\psi \rho_{\phi}\right)$. Hence it follows from (6.9) that

$$
\int\left|U^{* k} \psi\right| d \mu_{\phi}=\int\left|\hat{\mathcal{L}}_{\phi}^{k}\left(\psi \rho_{\phi}\right)\right| d m_{\phi} \preceq \xi^{k}\left\|\hat{\mathcal{L}}_{\phi}\left(\psi \rho_{\phi}\right)\right\|_{\beta} .
$$

By Theorem 6.16.

$$
\left|C_{k}(\psi, \psi)\right|=\left|\int \psi \psi \circ f^{k} d \mu_{\phi}\right| \preceq C \xi^{k}\left\|\hat{\mathcal{L}}_{\phi}\left(\psi \rho_{\phi}\right)\right\|_{\beta}\|\psi\|_{L^{1}\left(m_{\phi}\right)}
$$

for every $k \geq 1$. The $L^{1}$-convergence condition is thus verified.

If $\psi \in \mathcal{O}_{\beta}$ is in addition bounded then

$$
\left\|U^{* k} \psi\right\|_{L^{2}\left(\mu_{\phi}\right)}^{2}=<U^{k} U^{* k} \psi, \psi>\leq\|\psi\|_{\infty} \int U^{k}\left(\left|U^{* k} \psi\right|\right) d \mu_{\phi}=\|\psi\|_{\infty} \int\left|U^{* k} \psi\right| d \mu_{\phi}
$$

still by the $f$-invariance of $\mu_{\phi}$. The conclusion, that in this case the $L^{2}$-convergence holds, comes now from the preceding $L^{1}$-estimation. 
Proof of Theorem 6.17. Consider first the case of a centered bounded Hölder function $\psi \in H_{\beta}$. Then the $L^{2}$-convergence condition is satisfied and we are in the confortable situation where there exists a inverse Martingale approximation $\left(Y_{k}\right)_{k}$ with respect to the filtration $\mathcal{F}_{k}=f^{-k}\left(\mathcal{F}_{0}\right) \in L^{2}\left(\mu_{\phi}\right),\left(\mathcal{F}_{0}\right)$ the Borel $\sigma-$ algebra, and a function $b$ also in $L^{2}\left(\mu_{\phi}\right)$ such that

$$
U^{k} \psi=Y_{k}+U^{k} b-U^{k-1} b \quad, \quad k \geq 1 .
$$

The $Y_{k}$ being square integrable stationary and ergodic one knows that they satisfy CLT. Since

$$
\frac{1}{\sqrt{n}} S_{n} \Psi=\frac{1}{\sqrt{n}}\left(Y_{1}+\ldots+Y_{n}\right)+\frac{1}{\sqrt{n}}\left(U^{n} b-b\right)
$$

and since $\frac{1}{\sqrt{n}}\left(U^{n} b-b\right) \rightarrow 0$ as $n \rightarrow \infty$ one therefore has CLT for $\left(\psi \circ f^{k}\right)_{k}$. A direct calculation gives

$$
\sigma^{2}=E\left(Y_{1}^{2}\right)=\int \psi^{2} d \mu_{\phi}+2 \sum_{k=1}^{\infty} \int \psi \psi \circ f^{k} d \mu_{\phi}
$$

(see $[\mathbf{L i v}$ for details).

For general centered $\psi \in \mathcal{O}_{\beta}$ we have a good $L^{1}$-convergence (cf. Lemma 6.18) but $\psi$ is not bounded contrary to the assumption made by Liverani. His perturbation argument allows to get CLT but we only have an upper bound for the asymptotic variance. Let us briefly explain this. Firstly, the above function $b$ is given by $b=\sum_{k=0}^{\infty} U^{* k} \psi$. Therefore, the $L^{1}$-convergence condition yields $b \in L^{1}\left(\mu_{\phi}\right)$. In order to be able to work again in $L^{2}$ one introduces

$$
b_{\lambda}=\sum_{k=0}^{\infty} \lambda^{-k} U^{* k} \psi
$$

where $\lambda>1$. Clearly $b_{\lambda} \rightarrow b$ in $L^{1}\left(\mu_{\phi}\right)$. Set similarly

$$
Y_{\lambda, k}=U^{k} Y_{\lambda, 1}=U^{k} \psi-U^{k} b_{\lambda}+\lambda^{-1} U^{k-1} b_{\lambda} \in L^{2}\left(\mu_{\phi}\right) .
$$

The same direct calculation as before gives this time

$$
\sigma^{2}\left(Y_{\lambda, k}\right)=\sigma^{2}\left(Y_{\lambda, 1}\right) \leq \int \psi^{2} d \mu_{\phi}+2 \sum_{k=1}^{\infty} \lambda^{-k} \int \psi \psi \circ f^{k} d \mu_{\phi}
$$

and Fatou's Lemma yields for the asymptotic variance of $Y_{k}=\lim _{\lambda \rightarrow 1} Y_{\lambda, k}$

$$
\sigma^{2}\left(Y_{1}\right) \leq \liminf _{\lambda \rightarrow 1} \sigma^{2}\left(Y_{\lambda, 1}\right) \leq \int \psi^{2} d \mu_{\phi}+2 \sum_{k=1}^{\infty} \int \psi \psi \circ f^{k} d \mu_{\phi} .
$$

In particular the $\left(Y_{k}\right)_{k}$ are again in $L^{2}\left(\mu_{\phi}\right)$ and CLT holds. Let us conclude by mentioning that $\sigma^{2}=0$ clearly implies that $\psi \circ f=b \circ f-b$.

\subsection{Cohomologies and $\sigma^{2}=0$}

Let $\mathcal{F}$ be any class of real-valued functions defined on $\mathcal{J}(f)$. Two functions $\phi, \psi$ : $\mathcal{J}(f) \rightarrow \mathbb{R}$ are said to be cohomologous in the class of function $\mathcal{F}$ if there exists a function $u \in \mathcal{F}$ such that

$$
\phi-\psi=u-u \circ f
$$


Theorem 6.19. If $\phi, \psi: \mathcal{J}(f) \rightarrow \mathbb{R}$ are two arbitrary tame functions, then the following conditions are equivalent:

(1) $\mu_{\phi}=\mu_{\psi}$.

(2) There exists a constant $R$ such that for each $n \geq 1$, if $f^{n}(z)=z(z \in$ $\mathcal{J}(f))$, then

$$
S_{n} \phi(z)-S_{n} \psi(z)=n R
$$

(3) The difference $\psi-\phi$ is cohomologous to a constant $R$ in the class of Hölder continuous functions.

(4) The difference $\psi-\phi$ is cohomologous to a constant in the class of all functions defined everywhere in $\mathcal{J}(f)$ and bounded on bounded subsets of $\mathcal{J}(f)$.

If these conditions are satisfied, then $R=S=\mathrm{P}(\phi)-\mathrm{P}(\psi)$.

Proof. (1) $\Rightarrow(2)$. It follows from Theorem 6.5 and Lemma 5.20 that there exists a constant $C_{z} \geq 1$ (remember that $f^{n}(z)=z$ ) such that for every $k \geq 1$

$$
C_{z}^{-1} \exp \left(k S_{n} \phi(z)-\mathrm{P}(\phi) k n\right) \leq \mu_{\phi}\left(f_{z}^{-k n}(D(z, \delta))\right) \leq C_{z} \exp \left(k S_{n} \phi(z)-\mathrm{P}(\phi) k n\right)
$$

and

$$
C_{z}^{-1} \exp \left(k S_{n} \psi(z)-\mathrm{P}(\psi) k n\right) \leq \mu_{\psi}\left(f_{z}^{-k n}(D(z, \delta))\right) \leq C_{z} \exp \left(k S_{n} \psi(z)-\mathrm{P}(\psi) k n\right) .
$$

Since $\mu_{\phi}=\mu_{\psi}$, this gives that

$$
C_{z}^{-2} \leq \frac{\left.\exp \left(k S_{n} \phi(z)-\mathrm{P}(\phi) k n\right)\right)}{\left.\exp \left(k S_{n} \psi(z)-\mathrm{P}(\psi) k n\right)\right)} \leq C_{z}^{2}
$$

or equivalently

$$
C_{z}^{-2} \leq \exp \left(k\left(\left(S_{n} \phi(z)-S_{n} \psi(z)\right)-(\mathrm{P}(\phi)-\mathrm{P}(\psi)) n\right)\right) \leq C_{z}^{2}
$$

and

$$
-2 \log C_{z} \leq k\left(\left(S_{n} \phi(z)-S_{n} \psi(z)\right)-(\mathrm{P}(\phi)-\mathrm{P}(\psi)) n\right) \leq 2 \log C_{z} .
$$

Therefore, letting $k \nearrow \infty$, we conclude that $S_{n} \phi(z)-S_{n} \psi(z)=(\mathrm{P}(\phi)-\mathrm{P}(\psi)) n$. Thus, putting $R=\mathrm{P}(\phi)-\mathrm{P}(\psi)$ completes the proof of the implication (1) $\Rightarrow(2)$.

$(2) \Rightarrow(3)$. Define

$$
\eta=\phi-\psi-R
$$

Since the measure $\mu_{\phi}$ is ergodic and positive on non-empty open sets, the set of transitive points of $f$ has a full measure $\mu_{\phi}$. Fix a transitive point $w \in \mathcal{J}(f)$ and put

$$
\Gamma=\left\{f^{k}(w): k \geq 1\right\} .
$$

Define the function $\hat{u}: \Gamma \rightarrow \mathbb{R}$ by setting

$$
\hat{u}\left(f^{k}(w)\right)=\sum_{j=0}^{k-1} \eta\left(f^{j}(w)\right) .
$$

Let us first show that $\hat{u}$ is Hölder continuous. So, suppose that with some $1 \leq k<l$, the modulus $\left|f^{k}(w)-f^{l}(w)\right|<\delta$ is so small that

$$
l-k \geq \log \left(l C \delta^{-1}\right) / \log \gamma .
$$

Let $f_{*}^{-(l-k)}=f_{f^{k}(w)}^{-(l-k)}$ be the holomorphic inverse branch of $f^{l-k}$ defined on

$$
D\left(f^{l}(w), 4 \delta\right)
$$


and mapping $f^{l}(w)$ to $f^{k}(w)$. In view of the expanding property and (6.12) we have for every $z \in \bar{D}\left(f^{l}(w), 2 \delta\right)$ that

$\left|f_{*}^{-(l-k)}(z)-f^{l}(w)\right| \leq\left|f_{*}^{-(l-k)}(z)-f_{*}^{-(l-k)}\left(f^{l}(w)\right)\right|+\left|f^{k}(w)-f^{l}(w)\right| \leq C \gamma^{k-l}+\delta \leq 2 \delta$.

Thus $f_{*}^{-(l-k)}\left(\bar{D}\left(f^{l}(w), 2 \delta\right)\right) \subset \bar{D}\left(f^{l}(w), 2 \delta\right)$. Hence, in view of Brouwer's Fixed Point Theorem, there exists $y \in \bar{D}\left(f^{l}(w), 2 \delta\right)$ such that $f_{*}^{-(l-k)}(y)=y$. In particular, $f^{l-k}(y)=y$. Since for every $0 \leq i \leq l-k$ and for the map $f_{f^{l-i}(w)}^{-i}$ : $D\left(f^{l}(w), 4 \delta\right) \rightarrow \mathbb{C}$, we have $f_{f^{l-i}(w)}^{-i}=f^{l-k-i} \circ f_{*}^{-(l-k)}$, we obtain that

$$
f_{f^{l-i}(w)}^{-i}\left(f^{l-k}(y)\right)=f^{l-k-i}\left(f_{*}^{-(l-k)}\left(f^{l-k}(y)\right)\right)=f^{l-k-i}(y)
$$

or, in other words, for all $j=0,1, \ldots, l-k$,

$$
f^{j}(y)=f_{f^{k+j}(w)}^{-(l-k-j)}\left(f^{l-k}(y)\right)
$$

Since also

$$
f^{l}\left(f^{k}(w)\right)=f_{f^{k+j}(w)}^{-(l-k-j)}\left(f^{l}(w)\right)
$$

and since both $f^{l}(w)$ and $f^{l-k}(y)=y$ belong to $\bar{D}\left(f^{l}(w), 2 \delta\right)$, weak Hölderity of the function $\eta: \mathcal{J}(f) \rightarrow \mathbb{R}$ yields for all $0 \leq j \leq l-k$ that

$$
\begin{aligned}
\mid \varphi\left(f^{j}(y)\right)- & \varphi\left(f^{j}\left(f^{k}(w)\right)\right) \mid \leq \\
& \leq V_{\beta}(\varphi)\left|f_{f^{k+j-1}(w)}^{-(l-k-1))}\left(f^{l-k}(y)\right)-f_{f^{k+j-1}(w)}^{-(l-k-(j))}\left(f^{l}(w)\right)\right|^{\beta} \\
& \leq V_{\beta}(\varphi) K^{\beta}\left|\left(f^{l-k-j+1}\right)^{\prime}\left(f^{k+j-1}(w)\right)\right|^{-\beta}\left|f^{l-k}(y)-f^{l}(w)\right|^{\beta} \\
& \leq V_{\beta}(\varphi) c^{-\beta} \gamma^{-\beta(l-k-j+1)}\left|y-f^{l}(w)\right|^{\beta} .
\end{aligned}
$$

From (6.13) we get

$$
\left|y-f^{k}(w)\right| \leq K\left|\left(f^{l-k}\right)^{\prime}\left(f^{k}(w)\right)\right|^{-1}\left|f^{l-k}(y)-f^{l}(w)\right| \preceq \gamma^{-(l-k)} .
$$

Combining this with (6.14) we obtain

$$
\left|\varphi\left(f^{j}(y)\right)-\varphi\left(f^{j}\left(f^{k}(w)\right)\right)\right| \preceq V_{\beta}(\varphi) \gamma^{-\beta(l-k-j+1)}\left(\gamma^{-(l-k)}+\left|f^{k}(w)-f^{l}(w)\right|\right)^{\beta} .
$$

So we also get

$$
\left|\psi\left(f^{j}(y)\right)-\psi\left(f^{j}\left(f^{k}(w)\right)\right)\right| \preceq V_{\beta}(\varphi) \gamma^{-\beta(l-k-j+1)}\left(\gamma^{-(l-k)}+\left|f^{k}(w)-f^{l}(w)\right|\right)^{\beta}
$$


6. FINER PROPERTIES OF GIBBS STATES

for all $j=0,1, \ldots, l-k$. Hence, using our assumption (2) with $z$ replaced by $y$, we obtain

$$
\begin{aligned}
\mid \hat{u}\left(f^{l}(w)\right) & -\hat{u}\left(f^{k}(w)\right) \mid= \\
& =\left|\sum_{j=k}^{l-1} \eta\left(f^{j}(y)\right)\right|=\mid \sum_{j=0}^{l-k-1}\left(\eta\left(-f^{j}\left(f^{k}(w)\right)-\eta\left(f^{j}(y)\right)\right) \mid\right. \\
& \leq \sum_{j=0}^{l-k-1}\left(\left|\phi\left(f^{j}\left(f^{k}(w)\right)\right)-\phi\left(f^{j}(y)\right)\right|+\left|\psi\left(f^{j}\left(f^{k}(w)\right)\right)-\psi\left(f^{j}(y)\right)\right|\right) \\
& \preceq \sum_{j=0}^{l-k-1} \gamma^{\beta(l-k-j)}\left(\left|f^{k}(w)-f^{l}(w)\right|+\gamma^{k-l}\right)^{\beta} \\
& \preceq\left(\left|f^{k}(w)-f^{l}(w)\right|+\gamma^{k-l}\right)^{\beta} .
\end{aligned}
$$

Now, for every $z \in \mathcal{J}(f)$ put

$$
u(z)=\limsup _{\xi \rightarrow z ; \xi \in \Gamma} \hat{u}(\xi)
$$

In view of (6.15) $u(z)$ is a finite real number. It also follows from 6.15) and from the fact that $l-k \rightarrow \infty$ if $f^{l}(w) \rightarrow f^{k}(w)$ that

$$
u(z)=\hat{u}(z) \quad \text { for all } z \in \Gamma .
$$

Take now two arbitrary points $a, b \in \mathcal{J}(f)$ and suppose that $f^{m_{k}}(w) \rightarrow a$ and $f^{n_{k}}(w) \rightarrow b$. Passing to subsequences, we may assume without loss of generality that $n_{k}-m_{k} \rightarrow \infty$ and $\left|f^{m_{k}}(w)-f^{n_{k}}(w)\right|<\delta$. Passing to the limit $k \rightarrow \infty$ it then follows from (6.15) that

$$
|u(b)-u(a)| \preceq|b-a|^{\beta} .
$$

So, $u: \mathcal{J}(f) \rightarrow \mathbb{R}$ is $\beta$-Hölder and it follows from (6.16) together with the equality $\varphi(z)-\psi(z)-R=\hat{u}(z)-\hat{u}(f(z))$ for all $z$ in the dense set $\Gamma$ that $\varphi-\psi-R=u-u \circ f$ on $\mathcal{J}(f)$. The proof of the implication $(2) \Rightarrow(3)$ is complete.

The implication $(3) \Rightarrow(4)$ is obvious.

$(4) \Rightarrow(1)$. Fix $z \in J_{r, M}$, where the sets $J_{r, N}$ were defined at the beginning of the proof of Theorem 5.22 and $M>0$ is such that $m_{\phi}\left(J_{r, M}\right)=m_{\psi}\left(J_{r, M}\right)=1$ (Proposition 5.21). There then exists an unbounded increasing sequence $\left\{n_{k}\right\}_{k=1}^{\infty}$ such that $\left|f^{n_{k}}(z)\right| \leq M$ for all $k \geq 1$. Using Lemma 5.20 along with $\frac{1}{4}$-Koebe's distortion theorem and the standard version of Koebe's distortion theorem, we get that

$$
\begin{aligned}
m_{\phi}\left(B\left(z, \frac{1}{4} \delta\left|\left(f^{n_{k}}\right)^{\prime}(z)\right|^{-1}\right)\right) & \leq m_{\phi}\left(f_{z}^{-n_{k}}\left(B\left(f^{n_{k}}(z), \delta\right)\right)\right) \\
& \leq K_{\phi} \exp \left(S_{n_{k}} \phi(z)-\mathrm{P}(\phi) n_{k}\right) m_{\phi}\left(B\left(f^{n_{k}}(z), \delta\right)\right) \\
& \leq K_{\phi} \exp \left(S_{n_{k}} \phi(z)-\mathrm{P}(\phi) n_{k}\right)
\end{aligned}
$$


and

$$
\begin{aligned}
m_{\phi}\left(B\left(z, \frac{1}{4} \delta\left|\left(f^{n_{k}}\right)^{\prime}(z)\right|^{-1}\right)\right) \geq m_{\phi}\left(f_{z}^{-n_{k}}\left(B\left(f^{n_{k}}(z), \frac{1}{4} K^{-1} \delta\right)\right)\right) \\
\geq K_{\phi}^{-1} \exp \left(S_{n_{k}} \phi(z)-\mathrm{P}(\phi) n_{k}\right) m_{\phi}\left(B\left(f^{n_{k}}(z), \frac{1}{4} K^{-1} \delta\right)\right) \\
\geq K_{\phi}^{-1} Z_{\phi} \exp \left(S_{n_{k}} \phi(z)-\mathrm{P}(\phi) n_{k}\right)
\end{aligned}
$$

where $Z_{\phi}=\inf \left\{m_{\phi}\left(D\left(w,(4 K)^{-1} \delta\right)\right): w \in \mathcal{J}(f) \cap \bar{D}(0,2 M)\right\}$ is positive since the set $\mathcal{J}(f) \cap \bar{D}(0,2 M)$ is compact and the topological support of $m_{\phi}$ is equal to the Julia set $\mathcal{J}(f)$. Obviously, analogous inequalities hold for the potential $\psi$.

By (4) we know that there is a constant $S$ and a function $u$ such that $\phi-\psi=$ $S+u \circ f-u$. Since $u$ is locally bounded there is $T>0$ such that for every $z \in J_{r, M}$ and $n_{k}$ as above

$$
\left|S_{n_{k}}(\phi-\psi)(z)-n_{k} S\right| \leq T
$$

It follows that

$$
\begin{aligned}
\left(K_{\phi} C_{\psi} e^{T}\right)^{-1} Z_{\phi} & \exp \left((\mathrm{P}(\psi)-\mathrm{P}(\phi)+S) n_{k}\right) \leq \\
& \leq \frac{m_{\phi}\left(B\left(z, 4^{-1} \delta\left|\left(f^{n_{k}}\right)^{\prime}(z)\right|^{-1}\right)\right)}{m_{\psi}\left(B\left(z, 4^{-1} \delta\left|\left(f^{n_{k}}\right)^{\prime}(z)\right|^{-1}\right)\right)} \\
& \leq K_{\phi} C_{\psi} e^{T} Z_{\psi}^{-1} \exp \left((\mathrm{P}(\psi)-\mathrm{P}(\phi)+S) n_{k}\right) .
\end{aligned}
$$

Suppose that $S \neq \mathrm{P}(\phi)-\mathrm{P}(\psi)$. Without loss of generality we may assume that $S<\mathrm{P}(\phi)-\mathrm{P}(\psi)$. But then, using the right-hand side of (6.18), we conclude that $m_{\phi}\left(J_{r, M}\right)=0$. This contradiction shows that $S=\mathrm{P}(\phi)-\mathrm{P}(\psi)$. Then (6.18) implies that the measures $m_{\phi}$ and $m_{\psi}$ are equivalent on $J_{r, M}$. Since $m_{\phi}\left(J_{r, M}\right)=$ $m_{\psi}\left(J_{r, M}\right)=1$, these two measures are equivalent as considered on $\mathcal{J}(f)$. Since the measures $\mu_{\phi}$ and $\mu_{\psi}$ are ergodic and equivalent respectively to $m_{\phi}$ and $m_{\psi}$, we conclude that $\mu_{\phi}=\mu_{\psi}$. Thus the proof of the implication $(4) \Rightarrow(1)$, and therefore the entire proof of Theorem 6.19, is complete.

We can now prove that the case $\sigma^{2}=0$ in CLT (Theorem 6.17). is exceptional. Indeed, in the setting of rational functions and with $\psi=\log \left|f^{\prime}\right|$ this only can happen for some special functions, namely for Tchebychev polynomials, for $z \mapsto z^{d}$ and for Lattès maps ([ $\mathbf{Z d}]$, see also $\mathbf{M y 1}$ where a simplification of Zdunik's work is given). The following can be interpreted as a generalization of this fact to our class of meromorphic functions.

Theorem 6.20. If $\psi \in \bigcup_{\beta \in(0,1]} \mathcal{O}_{\beta}$ is a loosely $t$-tame function with $t \neq 0$, then

$$
\sigma_{\mu_{\phi}}^{2}(\psi)>0 \text {. }
$$

The proof goes in two steps. In the first one the regularity of the coboundary function is improved.

Proposition 6.21. If $\phi: \mathcal{J}(f) \rightarrow \mathbb{R}$ is a tame function and $\psi: \mathcal{J}(f) \rightarrow \mathbb{R}$ is a loosely tame function, then $\sigma_{\mu_{\phi}}^{2}(\psi)=0$ if and only if $\psi$ is cohomologous to a constant function in the class of Hölder continuous functions on $\mathcal{J}(f)$. 
Proof. We already know from Theorem 6.17 that $\sigma_{\mu_{\phi}}^{2}(\psi)=0$ if and only if there is a measurable function $u$ such that

$$
\psi=-t \log \left|f^{\prime}\right|_{\tau}+h=u-u \circ \tilde{f} \quad \mu_{\phi}-a . e .
$$

Our aim is to show that $u$ has a Hölder continuous version of order $s>0$ being a common Hölder exponent of $\phi$ and $\psi$.

In view of Luzin's theorem there exists a compact set $K \subset \mathcal{J}(f)$ such that $\mu_{\phi}(K)>1 / 2$ and the function $\left.u\right|_{K}$ is continuous. Consider a disk $D_{z}=D(z, \delta)$, $z \in \mathcal{J}(f)$. From Birkhoff's ergodic theorem (but here one has to work in the natural extension) follows that there exists a Borel set $B \subset D_{z} \cap \mathcal{J}(f)$ such that $\mu_{\phi}(B)=\mu_{\phi}\left(D_{z}\right)$ and for every $x \in B f_{*}^{-n}(x)$ visits $K$ with the asymptotic frequence $>1 / 2$ where $f_{*}^{-n}$ denotes any inverse branch of $f^{n}$ defined on $D_{z}$. Consider two arbitrary elements $\rho, \tau \in B$. Then there exists an unbounded increasing sequence $\left\{n_{j}\right\}$ such that $f_{*}^{-n_{j}}(\rho), f_{*}^{-n_{j}}(\tau) \in K$ for all $j \geq 1$. Using (6.19) we get

$$
|u(\rho)-u(\tau)| \leq\left|u\left(f_{*}^{-n_{j}}(\rho)\right)-u\left(f_{*}^{-n_{j}}(\tau)\right)\right|+\left|S_{n_{j}} \psi\left(f_{*}^{-n_{j}}(\rho)\right)-S_{n_{j}} \psi\left(f_{*}^{-n_{j}}(\tau)\right)\right| .
$$

Now, since $\lim _{j \rightarrow \infty} \operatorname{dist}\left(f_{*}^{-n_{j}}(\rho), f_{*}^{-n_{j}}(\tau)\right)=0$, since both $f_{*}^{-n_{j}}(\rho)$ and $f_{*}^{-n_{j}}(\tau)$ belong to $K$ and since $\left.u\right|_{K}$ is uniformly continuous (as $K$ is compact), we conclude that

$$
\lim _{j \rightarrow \infty}\left|u\left(f_{*}^{-n_{j}}(\rho)\right)-u\left(f_{*}^{-n_{j}}(\tau)\right)\right|=0 .
$$

Hölder continuity of $u_{\mid B}$ results now from the distortion property of Lemma 5.2 The assertion follows then from this continuity together with the density of $B$ in $D_{z} \cap \mathcal{J}(f)$.

Proof of Theorem 6.20, The following fact is proven in Bw2: if $f$ is any transcendental entire function, then $f^{2}$ has infinitely many repelling fixed points.

Let us show the same statement for a hyperbolic transcendental meromorphic (and non-entire) function. Such a function $f$ has a pole $b$ which is not an asymptotic value. Consequently $f^{-1}(b)$ is an infinite set and, using Brower's fixed point Theorem, it is then easy to construct a sequence of fixed points $p_{n}$ for $f^{2}$.

In both cases, entire and meromorphic, it follows from the growth condition that

$$
\left|\left(f^{2}\right)^{\prime}\left(p_{n}\right)\right| \rightarrow \infty \quad \text { if } n \rightarrow \infty .
$$

Suppose now that $\sigma_{\mu_{\phi}}^{2}(\psi)=0$ where $\psi=-t \log \left|f^{\prime}\right|_{\tau}+h$ is a loosely tame potential with $t \neq 0$. Then Proposition 6.21 yield that there is $u$ continuous such that

$$
\psi-c=u-u \circ f
$$

for some constant $c$. But this leads to

$$
0=u\left(p_{n}\right)-u\left(f^{2}\left(p_{n}\right)\right)=-t \log \left|\left(f^{2}\right)^{\prime}\left(p_{n}\right)\right|_{\tau}+h\left(p_{n}\right)+h\left(f\left(p_{n}\right)\right)-2 c
$$

for all $n \geq 1$. Since $h$ is bounded we therefore have a contradiction to (6.20). 


\subsection{Variational Principle}

In this section we give a variational characterization of Gibbs states of tame potentials and dynamically semiregular meromorphic functions, which is very close to the classical one. We begin by proving the following general lemma.

Recall that given a countable partition $\mathcal{P}$ of $\mathcal{J}(f)$, for every $x \in \mathcal{J}(f)$ we denote by $\mathcal{P}(x)$ the only element of $\mathcal{P}$ containing $x$. Given in addition $1 \leq n \leq+\infty$, we put

$$
\mathcal{P}^{n}=\bigvee_{j=0}^{n-1} f^{-j}(\mathcal{P}) .
$$

If also a Borel probability $f$-invariant measure $\mu$ is given, the partition $\mathcal{P}$ is said to be generating for $\mu$ provided that for $\mu$-a.e. $x \in \mathcal{J}(f)$ the set $\mathcal{P}^{\infty}(x)$ is a singleton. We start with the following.

Lemma 6.22. If $f$ is a dynamically semi-regular meromorphic function, $\phi: \mathcal{J}(f) \rightarrow$ $\mathbb{R}$ is a loosely tame potential and $\mu$ is a Borel probability $f$-invariant measure on $\mathcal{J}(f)$ with respect to which the function $\phi$ is integrable, then also the functions $\log \left|f^{\prime}(z)\right|_{\tau}, \log \left|f^{\prime}(z)\right|$, and $\log |z|$ are integrable.

Proof. Integrability of the function $\log \left|f^{\prime}(z)\right|_{\tau}$ follows immediately from integrablility of $\phi$ since $\|\phi+\theta(\phi)\|_{\infty}$ is finite. From (3.4) we get that

$$
\log \left|f^{\prime}(z)\right|_{\tau}=-\tau \log |f(z)|+\log \left|f^{\prime}(z)\right|+\tau \log |z| \geq\left(\underline{\alpha}_{2}-\tau\right) \log |f(z)|+\hat{\tau} \log |z| .
$$

Since $\underline{\alpha}_{2}>\tau$ and both functions $\log |z|$ and $\log |f(z)|$ are uniformly bounded below, we thus conclude that both $\log |z|$ and $\log |f(z)|$ are integrable. Consequently, the first part of (6.21) yields that $\log \left|f^{\prime}(z)\right|$ is integrable.

Endow now the extended complex plane $\hat{\mathbb{C}}$ with the spherical metric and denote by $\operatorname{diam}_{s}(A)$ the spherical diameter of any subset $A$ of $\hat{\mathbb{C}}$. Since, under the assumptions of Lemma 6.22. the logarithm of the function $z \mapsto C|z|^{-2}\left|f^{\prime}(z)\right|^{-1}$ is $\mu$-integrable for every $C>0$ and since $\hat{\mathbb{C}}$ with the spherical metric is a compact Riemannian manifold, as a direct consequence of Mane's Theorem (see Lemma 13.3 in Mane] we have the following.

Lemma 6.23. With the assumptions of Lemma 6.22, for every constant $C>0$ there exists a countable partition $\mathcal{P}_{\mu}$ of $\mathcal{J}(f)$ into Borel sets with the following properties.

(a) $\mathrm{H}_{\mu}\left(\mathcal{P}_{\mu}\right)<+\infty$, where $\mathrm{H}_{\mu}\left(\mathcal{P}_{\mu}\right)=\sum_{P \in \mathcal{P}_{\mu}}-\mu(P) \log \mu(P)$ is the entropy of the partition $\mathcal{P}_{\mu}$.

(b) $\operatorname{diam}_{s}\left(\mathcal{P}_{\mu}(z)\right) \leq C|z|^{-2}\left|f^{\prime}(z)\right|^{-1}$ for $\mu$-a.e. $z \in \mathcal{J}(f)$.

For every Borel probability $f$-invariant measure $\mu$ let $J_{\mu}: \mathcal{J}(f) \rightarrow[1,+\infty]$ be the (weak) Jakobian of the measure $\mu$, i.e.

$$
\mu\left(f_{z}^{-1}(A)\right)=\int_{A} \mathcal{J}_{\mu}^{-1}\left(f_{z}^{-1}(\xi)\right) d \mu(\xi)
$$


for every $z \in \mathcal{J}(f)$ and every Borel set $A \subset D(f(z), 2 \delta)$. As a consequence of Lemma 6.23 (see $\mathbf{P y}$, comp. [PU]), we get the following.

Lemma 6.24. With the assumptions of Lemma 6.22, $\mathrm{h}_{\mu}(f)=\int \log J_{\mu} d \mu$.

The main result of this section is the following.

Theorem 6.25 (Variational Principle). If $f: \mathbb{C} \rightarrow \mathbb{C}$ is dynamically semi-regular and if $\phi: \mathcal{J}(f) \rightarrow \mathbb{C}$ is a tame potential, then the invariant measure $\mu_{\phi}$ is the only equilibrium state of the potential $\phi$, that is

$$
\mathrm{P}(\phi)=\sup \left\{\mathrm{h}_{\mu}(f)+\int \phi d \mu\right\}
$$

where the supremum is taken over all Borel probability $f$-invariant ergodic measures $\mu$ with $\int \phi d \mu>-\infty$, and

$$
\mathrm{P}(\phi)=\mathrm{h}_{\mu_{\phi}}+\int \phi d \mu_{\phi}
$$

Proof. We shall show first that

$$
\mathrm{h}_{\mu_{\phi}}+\int \phi d \mu_{\phi} \geq \mathrm{P}(\phi) .
$$

Indeed, fix $C>0$ so small that if $|z| \geq T, z \in A \cap \mathcal{J}(f)$ and $\operatorname{diam}_{s}(A) \leq$ $C|z|^{-2}\left|f^{\prime}(z)\right|^{-1}$, then $A \subset D\left(z, \delta\left|f^{\prime}(z)\right| / 4\right)$. Since $\int|\phi| d \mu_{\phi}<+\infty$, we have the partition $\mathcal{P}=\mathcal{P}_{\mu_{\phi}}$ given by Lemma 6.23. Since, by Koebe's $\frac{1}{4}$-Distortion Theorem and Lemma 6.23(b), $f_{z}^{-1}(D(f(z), \delta)) \subset D\left(z, \delta\left|f^{\prime}(z)\right| / 4\right)$, for $\mu_{\phi^{-}}$-a.e. $z \in \mathcal{J}(f)$, we conclude that the restriction $\left.f\right|_{\mathcal{P}(z)}$ is injective, and consequently,

$$
\mathcal{P}^{n}(z) \subset f_{z}^{-n}\left(D\left(f^{n}(z), \delta\right)\right)
$$

for $\mu$-a.e $z \in \mathcal{J}(f)$ and all $n \geq 1$. Since $\lim _{n \rightarrow \infty} \operatorname{diam}\left(f_{z}^{-n}\left(D\left(f^{n}(z), \delta\right)\right)\right)=0$, we thus see that each element of partition $\mathcal{P}^{\infty}$ is a singleton, meaning that the partition $\mathcal{P}$ is generating for the measure $\mu_{\phi}$. Applying Birkhoff's Ergodic Theorem and the Breiman-McMillan-Shanon Theorem for the $f$-invariant measure $\mu_{\phi}$ and utilizing Lemma 5.20 along with Theorem $5.15(4)$, we therefore get for $\mu_{\phi}$-a.e. $x \in \mathcal{J}(f)$ that

$$
\begin{aligned}
-\mathrm{h}_{\mu_{\phi}} & =\lim _{n \rightarrow \infty} \frac{1}{n} \log \left(\mu_{\phi}\left(\mathcal{P}^{n}(z)\right)\right) \\
& \leq \liminf _{n \rightarrow \infty} \frac{1}{n} \log \mu_{\phi}\left(f_{x}^{-n}\left(D\left(f^{n}(x), \delta\right)\right)\right) \\
& \preceq \liminf _{n \rightarrow \infty} \frac{1}{n}\left(\log \left(2 \rho_{\phi}(x)\right)+S_{n} \phi(x)-\mathrm{P}(\phi) n\right) \\
& =\lim _{n \rightarrow \infty} \frac{1}{n} S_{n} \phi(x)-\mathrm{P}(\phi)=\int \phi d \mu_{\phi}-\mathrm{P}(\phi) .
\end{aligned}
$$

Formula (6.22) is proved.

We now shall prove the following.

Claim 1: If $\mu$ is an ergodic $f$-invariant Borel probability measure on $\mathcal{J}(f)$ such that $\int \phi d \mu>-\infty$, then $\mathrm{h}_{\mu}(f)+\int \phi d \mu \leq \mathrm{P}(\phi)$; if in addition $\mu$ is an equilibrium state for $\phi$ and $f$ then $J_{\mu}=\frac{\rho_{\phi} \circ T}{\rho_{\phi}} \cdot \exp (\mathrm{P}(\phi)-\phi) \mu$ almost everywhere on $\mathcal{J}(f)$. 
Proof. Let $\mathcal{L}_{\mu}: L^{\infty}(\mu) \rightarrow L^{\infty}(\mu)$ be the Perron-Frobenius operator associated to the measure $\mu$. $\mathcal{L}_{\mu}$ is determined by the formula

$$
\mathcal{L}_{\mu}(g)(x)=\sum_{y \in f^{-1}(x)} J_{\mu}^{-1}(y) g(y) .
$$

Using Theorem 6.5 the $f$-invariance of $\mu$ and Lemma 6.24, we can write

$$
\begin{aligned}
1 & =\int \mathbb{1} d \mu=\int \frac{\hat{\mathcal{L}}_{\phi} \rho_{\phi}}{\rho_{\phi}} d \mu \\
& =\int \mathcal{L}_{\mu}\left(\frac{\rho_{\phi} \cdot \exp (\phi-\mathrm{P}(\phi))}{J_{\mu}^{-1} \cdot \rho_{\phi} \circ f}\right) d \mu \\
& =\int \frac{\rho_{\phi} \cdot \exp (\phi-\mathrm{P}(\phi))}{J_{\mu}^{-1} \cdot \rho_{\phi} \circ f} d \mu \geq 1+\int \log \left(\frac{\rho_{\phi} \cdot \exp (\phi-P(\phi))}{J_{\mu}^{-1} \cdot \rho_{\phi} \circ f}\right) d \mu \\
& =1+\int \log \rho_{\phi} d \mu-\int \log \rho_{\phi} \circ f d \mu+\int(\phi-\mathrm{P}(\phi)) d \mu+\int \log J_{\mu} d \mu \\
& =1+\int \phi d \mu-\mathrm{P}(\phi)+h_{\mu}(f) .
\end{aligned}
$$

Therefore $h_{\mu}(f)+\int \phi d \mu \leq \mathrm{P}(\phi)$ If $\mathrm{P}(\phi)=h_{\mu}(f)+\int \phi d \mu$, we can extend the last line of (6.23) by writing $1+\int \phi d \mu-\mathrm{P}(\phi)+h_{\mu}(f)=1$. Hence, the " $\geq$ " in the third line of (6.23) becomes an equality sign, and we get $\frac{\rho_{\phi} \cdot \exp (\phi-P(\phi))}{J_{\mu}^{-1} \cdot \rho_{\phi} \circ f}=1 \mu$ a.e. We are done with Claim 1.

Thus, we are left to show that $\mu_{\phi}$ is a unique equilibrium state for $\phi$. We need the following.

Claim 2: Any ergodic equilibrium state $\mu$ for $f$ and $\phi$ is absolutely continuous with respect to $\mu_{\phi}$.

Proof. For all integers $l, l \geq p:=\max \{1, \delta\}$ let $J_{r, k, l}(f)$ be the set of all those points in $\mathcal{J}(f) \cap D(0, k)$ whose $\omega$-limit set intersects $D(0, l)$. Since the measure $\mu$ is ergodic, $\mu\left(J_{r}(f)=\bigcup_{k, l>p} J_{r, k, l}(f)\right)=1$, and in order to prove our claim it suffices to show that for all $k, l \geq p$ there exists $C_{k, l}>0$ such that

$$
\mu(A) \leq C_{k, l} m_{\phi}(A)
$$

for every Borel set $A \subset J_{r, k, l}(f)$. Indeed, take an arbitrary point $\in J_{r, k, l}(f)$. There then exists an unbounded increasing sequence $\left(n_{j}\right)_{j=1}^{\infty}$ such that $f^{n_{j}}(z) \in D(0, l)$ for all $j \geq 1$. Put

$$
r_{j}(z)=\frac{1}{4} \delta\left|\left(f^{n_{j}}\right)^{\prime}(z)\right|^{-1}
$$

It follows from $\frac{1}{4}$-Koebe's Distortion Theorem that $D\left(z, r_{j}(z)\right) \subset f_{z}^{-n_{j}}\left(D\left(f^{n_{j}}(z), \delta\right)\right)$ and applying Lemma 5.3 along with Claim 1, we get with $G_{\phi}:=\inf \left\{\rho_{\phi}(\xi): \xi \in\right.$ $D(0,2 k)\}>0$, that

$$
\begin{aligned}
\mu\left(D\left(z, r_{j}(z)\right)\right) & \leq \frac{\left\|\rho_{\phi}\right\|_{\infty}}{G_{\phi}} c_{\phi} \exp \left(S_{n_{j}} \phi(z)-\mathrm{P}(\phi) n_{j}\right) \mu\left(D\left(f^{n_{j}}(z), \delta\right)\right) \\
& \leq\left\|\rho_{\phi}\right\|_{\infty} G_{\phi}^{-1} c_{\phi} \exp \left(S_{n_{j}} \phi(z)-\mathrm{P}(\phi) n_{j}\right) .
\end{aligned}
$$


On the other hand, it follows from Koebe's Distortion Theorem that $D\left(z, r_{j}(z)\right) \supset$ $f_{z}^{-n_{j}}\left(D\left(f^{n_{j}}(z),(4 K)^{-1} \delta\right)\right)$, and therefore, apllying Lemma 5.20

$$
\begin{aligned}
m_{\phi}\left(D\left(z, r_{j}(z)\right)\right) & \geq K_{\phi}^{-1} c_{\phi} \exp \left(S_{n_{j}} \phi(z)-\mathrm{P}(\phi) n_{j}\right) \mu\left(D\left(f^{n_{j}}(z),(4 K)^{-1} \delta\right)\right) \\
& \leq K_{\phi}^{-1} c_{\phi} M_{l} \exp \left(S_{n_{j}} \phi(z)-\mathrm{P}(\phi) n_{j}\right),
\end{aligned}
$$

where $M-l=\inf \left\{m_{\phi}\left(D\left(\xi,(4 K)^{-1} \delta\right)\right): \xi \in D(0, l)\right\}>0$. Combining this and (6.25), we get that

$$
\mu\left(D\left(z, r_{j}(z)\right)\right) \leq K_{\phi} c_{\phi}^{2} G_{\phi}^{-1}\left\|\rho_{\phi}\right\|_{\infty} M_{l}^{-1} m_{\phi}\left(D\left(z, r_{j}(z)\right)\right) .
$$

Using now Besicovic Covering Theorem, (6.24) follows in the same way as that employed in Theorem 5.22. We are done with Claim 2.

Now, the conclusion of the proof of Theorem 6.25 is straightforward. Since any two ergodic invariant measures are either equal or mutually singular, it follows form Claim 2 that $\mu_{\phi}$ is the only ergodic equilibrium state for $\phi$ and we are done.

As is a first useful application of the variational principle we see that the particular choice of the metric $\sigma_{\tau}$ does not influence the pressure.

Proposition 6.26. If $f: \mathbb{C} \rightarrow \mathbb{C}$ is dynamically semi-regular and if

$$
\phi_{\tau}=-t \log \left|f^{\prime}\right|_{\tau}+h: \mathcal{J}(f) \rightarrow \mathbb{C}
$$

is a tame potential, then the pressure $\mathrm{P}\left(\phi_{\tau}\right)$ does not depend on $\tau$.

Proof. Let $\tau_{1} \neq \tau_{2}$. The conformal measures $m_{\phi_{\tau_{1}}}, m_{\phi_{\tau_{2}}}$ are related by

$$
|z|^{\tau_{1}} d m_{\phi_{\tau_{1}}}=|z|^{\tau_{2}} d m_{\phi_{\tau_{2}}} \text {. }
$$

In particular they are mutually absolutely continuous. Since by Theorem 5.15 we have unicity of the corresponding Gibbs states $\mu_{\phi_{\tau_{1}}}, \mu_{\phi_{\tau_{2}}}$ they must coincide. Call $\mu=\mu_{\phi_{\tau_{1}}}=\mu_{\phi_{\tau_{2}}}$ this invariant measure. It follows then from the variational principle, the integrability of $\log |z|, \log |f(z)|$ (see Lemma 7.11 or MyU2, Lemma $8.2])$ together with the $f$-invariance of $\mu$ that

$$
\begin{aligned}
\mathrm{P}\left(\phi_{\tau_{1}}\right) & =h_{\mu}(f)+\int \phi_{\tau_{1}} d \mu \\
& =h_{\mu}(f)+\int \phi_{\tau_{2}} d \mu+t\left(\tau_{2}-\tau_{1}\right) \int(\log |z|-\log |f(z)|) d \mu \\
& =\mathrm{P}\left(\phi_{\tau_{2}}\right) .
\end{aligned}
$$




\section{CHAPTER 7}

\section{Regularity of Perron-Frobenius Operators and Topological Pressure}

\subsection{Analyticity of Perron-Frobenius Operators}

In this section we prove one main theorem about analyticity of Perron-Frobenius operators of tame potentials and then we derive some of its first consequences. Further application will come up in subsequent sections and chapters. For every $\xi \in \mathcal{J}(f)$ set

$$
\mathrm{H}_{\beta, \xi}=\left\{g: D(\xi, \delta) \rightarrow \mathbb{C}:\|g\|_{\beta}:\|g\|_{\infty}+V_{\beta, \xi}(g)<+\infty\right\},
$$

where $V_{\beta, \xi}$ comes from (4.8). Obviously \|\|$_{\beta}$ is a norm on $\mathrm{H}_{\beta, \xi}$ and $\mathrm{H}_{\beta, \xi}$ endowed with this norm becomes a banach space. For every function $F: G \rightarrow L\left(\mathrm{H}_{\beta}\right)$ and every $\xi \in \mathcal{J}(f)$ define the function $F_{\xi}: G \rightarrow L\left(\mathrm{H}_{\beta}, \mathrm{H}_{\beta, \xi}\right)$ by the formula

$$
F_{\xi}(\lambda) \psi=\left.(F(\lambda) \psi)\right|_{D(\xi, \delta)},
$$

where $L\left(\mathrm{H}_{\beta}, \mathrm{H}_{\beta, \xi}\right)$ is the Banach space of bounded linear operators from $\mathrm{H}_{\beta}$ to $\left.\mathrm{H}_{\beta, \xi}\right)$. We start with the following.

Lemma 7.1. Let $G$ be an open subset of a complex plane $\mathbb{C}$ and fix a function $F: G \rightarrow \mathcal{L}\left(\mathrm{H}_{\beta}\right)$. If for every $\xi \in \mathcal{J}(f)$ the function $F_{\xi}: G \rightarrow L\left(\mathrm{H}_{\beta}, \mathrm{H}_{\beta, \xi}\right)$ is analytic and $\sup \left\{\left\|F_{\xi}(\lambda)\right\|_{\beta}: \xi \in \mathcal{J}(f), \lambda \in G\right\}<+\infty$, then the function $F: G \rightarrow \mathcal{L}\left(\mathrm{H}_{\beta}\right)$ is analytic.

Proof. Fix $\lambda^{0} \in G$ and take $r>0$ so small that $D\left(\lambda^{0}, r\right) \subset G$. Then for each $\xi \in \mathcal{J}(f)$

$$
F_{\xi}(\lambda)=\sum_{n=0}^{\infty} a_{\xi, n}\left(\lambda-\lambda^{0}\right)^{n}, \quad \lambda \in D\left(\lambda^{0}, r\right)
$$

with some $a_{\xi, n} \in L\left(\mathrm{H}_{\beta}, \mathrm{H}_{\beta, \xi}\right)$. Put $M=\sup \left\{\left\|F_{\xi}(\lambda)\right\|_{\beta}: \xi \in \mathcal{J}(f): \lambda \in G\right\}<+\infty$. It follows from Cauchy's estimates that

$$
\left\|a_{\xi, n}\right\|_{\beta} \leq M r^{-n} .
$$

Now for every $n \geq 0$ and every $g \in \mathrm{H}_{\beta}$, set

$$
a_{n}(g)(z)=a_{z, n}(g)(z), \quad z \in \mathcal{J}(f) .
$$

Then

$$
\left\|a_{n} g\right\|_{\infty} \leq\left\|a_{z, n}\right\|_{\infty}\|g\|_{\infty} \leq\left\|a_{z, n}\right\|\left\|_{\beta}\right\| g \|_{\beta}
$$


Now, if $|z-\xi|<\delta$, then for every $g \in \mathrm{H}_{\beta}$ and every $w \in D(\xi, \delta) \cap D(z, \delta)$,

$$
\begin{aligned}
\sum_{n=0}^{\infty} a_{\xi, n}(g)(w)\left(\lambda-\lambda^{0}\right)^{n} & =\left(F_{\xi}(\lambda) g\right)(w)=F(\lambda) g(w)=\left(F_{z}(\lambda) g\right)(w) \\
& =\sum_{n=0}^{\infty} a_{z, n}(g)(w)\left(\lambda-\lambda^{0}\right)^{n}
\end{aligned}
$$

for all $\lambda \in D\left(\lambda^{0}, r\right)$. The uniqueness of coefficients of Taylor series expansion implies that for all $n \geq 0$,

$$
a_{\xi, n}(g)(w)=a_{z, n}(g)(w) .
$$

Since $\xi, z \in D(\xi, \delta) \cap D(z, \delta)$, we thus get, using (7.1),

$$
\begin{aligned}
\left|a_{n}(g)(z)-a_{n}(g)(\xi)\right| & =\left|a_{z, n}(g)(z)-a_{\xi, n}(g)(\xi)\right|=\left|a_{\xi, n}(g)(z)-a_{\xi, n}(g)(\xi)\right| \\
& \leq\left\|a_{\xi, n}(g)\right\|\left\|\left._{\beta}\left|\xi-z\left\|^{\beta} \leq\right\| a_{\xi, n}\right|\right|_{\beta}|| g\right\|_{\beta} \mid \xi-z \|^{\beta} \\
& \leq M r^{-n}|| g\left\|_{\beta} \mid \xi-z\right\|^{\beta} .
\end{aligned}
$$

Consequently, $v_{\beta}\left(a_{n}(g)\right) \leq M r^{-n}\|g\|_{\beta}$. Combining this with (7.2), we obtain $\left\|a_{n}(g)\right\|_{\beta} \leq 2 M r^{-n}\|g\|_{\beta}$. Thus $a_{n} \in L\left(\mathrm{H}_{\beta}\right)$ and $\left\|a_{n}\right\|_{\beta} \leq 2 M r^{-n}$. Thus the series

$$
\sum_{n=0}^{\infty} a_{n}\left(\lambda-\lambda^{0}\right)^{n}
$$

converges absolutely uniformly on $D\left(\lambda^{0}, r / 2\right)$ and $\left\|\sum_{n=0}^{\infty} a_{n}\left(\lambda-\lambda^{0}\right)^{n}\right\|_{\beta} \leq 2 M$ for all $\lambda \in D\left(\lambda^{0}, r / 2\right)$. Finally, for every $g \in \mathrm{H}_{\beta}$ and every $z \in \mathcal{J}(f)$,

$$
\begin{aligned}
\left(\sum_{n=0}^{\infty} a_{n}\left(\lambda-\lambda^{0}\right)^{n}\right) g(z) & =\sum_{n=0}^{\infty} a_{n}(g)(z)\left(\lambda-\lambda^{0}\right)^{n}=\sum_{n=0}^{\infty} a_{n}(g)(z)\left(\lambda-\lambda^{0}\right)^{n} \\
& =\left(\sum_{n=0}^{\infty} a_{z, n}\left(\lambda-\lambda^{0}\right)^{n}\right) g(z)=F_{z}(\lambda) g(z) \\
& =(F(\lambda) g)(z) .
\end{aligned}
$$

So, $F(\lambda) g=\left(\sum_{n=0}^{\infty} a_{n}\left(\lambda-\lambda^{0}\right)^{n}\right) g$ for all $g \in \mathrm{H}_{\beta}$, and consequently, $F(\lambda)=$ $\sum_{n=0}^{\infty} a_{n}\left(\lambda-\lambda^{0}\right)^{n}, \lambda \in D\left(\lambda^{0}, r / 2\right)$. We are done.

The main technical result of this section is the following.

Theorem 7.2. Suppose that $G$ is an open subset of a complex space $\mathbb{C}^{d}$ with some $d \geq 1$. Suppose also that for every $\lambda \in G, \phi_{\lambda}=-t_{\lambda} \log \left|f^{\prime}\right|_{\tau}+h_{\lambda}: \mathcal{J}(f) \rightarrow \mathbb{C}$ is a $\beta$-Hölder loosely tame potential and the following conditions are satisfied.

(a) $\sup \left\{\left\||| h_{\lambda} \mid\right\|_{\beta}: \lambda \in G\right\}<\infty$.

(b) The function $\lambda \mapsto t_{\lambda}(z), \lambda \in G$, is holomorphic.

(c) For every $z \in \mathcal{J}(f)$ the function $\lambda \mapsto h_{\lambda}(z), \lambda \in G$, is holomorphic.

(d) $\inf \left\{\operatorname{Re}\left(t_{\lambda}\right): \lambda \in G\right\}>\rho / \hat{\tau}$.

Then all the potentials $\phi_{\lambda}, \lambda \in G$, are tame and the map $\lambda \mapsto \mathcal{L}_{\phi_{\lambda}} \in L\left(\mathrm{H}_{\beta}\right), \lambda \in G$, is holomorphic.

Proof. By (d) all the potentials $\phi_{\lambda}, \lambda \in G$, are tame. Put

$$
H=\sup \left\{||\left|h_{\lambda}\right| \|_{\beta}: \lambda \in G\right\}<\infty \text { and } l=\inf \left\{\operatorname{Re}\left(t_{\lambda}\right): \lambda \in G\right\}>\rho / \tilde{\tau} .
$$


We therefore get for for every $\lambda \in G$ and every $v \in \mathcal{J}(f)$ that

$$
\left\|\exp \left(\phi_{\lambda} \circ f_{v}^{-1}\right)\right\|_{\infty} \leq e^{H}\left|f^{\prime}(v)\right|_{\tau}^{-l} .
$$

In virtue of Hartogs Theorem we may assume without loss of generality that $d=1$, i.e. $G \subset \mathbb{C}$. Now fix $\lambda^{0} \in G$ and take a radius $r>0$ so small that $\bar{D}\left(\lambda^{0}, r\right) \subset G$. In view of (b) and (c), the function $\lambda \mapsto \exp \left(\phi_{\lambda} \circ f_{v}^{-1}(z)\right)$ is holomorphic for every $z \in D(f(v), \delta)$. Consider its Taylor series expansion

$$
\exp \left(\phi_{\lambda} \circ f_{v}^{-1}(z)\right)=\sum_{n=0}^{\infty} a_{v, n}(z)\left(\lambda-\lambda^{0}\right)^{n}, \quad \lambda \in D\left(\lambda^{0}, r\right) .
$$

In view of Cauchy's estimates and (7.3) we get

$$
\left|a_{v, n}(z)\right| \leq e^{H}\left|f^{\prime}(v)\right|_{\tau}^{-l} r^{-n}
$$

and, using in addition Lemma 5.3 .

$$
\begin{aligned}
\left|a_{v, n}(w)-a_{v, n}(z)\right| & \leq r^{-n}\left|\exp \left(\phi_{\lambda} \circ f_{v}^{-1}(w)\right)-\exp \left(\phi_{\lambda} \circ f_{v}^{-1}(z)\right)\right| \\
& \leq c(\beta, c(\beta, v(\phi)))\left|\exp \left(\phi_{\lambda} \circ f_{v}^{-1}(z)\right)\right| r^{-n}|w-z|^{\beta} \\
& \leq \hat{c}\left|f^{\prime}(v)\right|_{\tau}^{-l} r^{-n}|w-z|^{\beta},
\end{aligned}
$$

where $\hat{c}=e^{H} c\left(\beta, H+v\left(\log \left|f^{\prime}(v)\right|_{\tau}\right)\right) \sup \left\{\left|t_{\lambda}\right|: \lambda \in \bar{D}\left(\lambda^{0}, r\right)\right\}$. Take an arbitrary $g \in \mathrm{H}_{\beta}$ and consider the product $a_{v, n}(z) g\left(f_{v}^{-1}(z)\right)$. By (7.4) we get

$$
\left|a_{v, n}(z) g\left(f_{v}^{-1}(z)\right)\right| \leq e^{H}\left|f^{\prime}(v)\right|_{\tau}^{-l} r^{-n}|| g \|_{\infty},
$$

and, in view of (7.5) and (7.4), we obtain

$$
\begin{aligned}
\mid a_{v, n}(w) g\left(f_{v}^{-1}(w)\right) & -a_{v, n}(z) g\left(f_{v}^{-1}(z)\right) \mid \leq \\
& \leq\left.\left|a_{v, n}(w)-a_{v, n}(z)\right| \cdot|| g\right|_{\infty}+\left|a_{v, n}(z)\right||| g \|_{\beta} L^{\beta} \gamma^{-\beta}|w-z|^{\beta} \\
& \leq\left|f^{\prime}(v)\right|_{\tau}^{-l} r^{-n}\left(\hat{c}+e^{H} L^{\beta} \gamma^{-\beta}\right)|| g||_{\beta}|w-z|^{\beta} \\
& =\hat{c}_{1}\left|f^{\prime}(v)\right|_{\tau}^{-l} r^{-n}|| g \|_{\beta}|w-z|^{\beta},
\end{aligned}
$$

where $\hat{c}_{1}=\hat{c} 1+e^{H} L^{\beta} \gamma^{-\beta}$. Combining this and (7.6) we conclude that the formula $N_{v, n} g(z)=a_{v, n}(z) g\left(f_{v}^{-1}(z)\right)$ defines a bounded linear operator $N_{v, n}: \mathrm{H}_{\beta} \rightarrow \mathrm{H}_{\beta, \xi}$, where $\xi=f(z)$, and

$$
\left\|N_{v, n}\right\|_{\beta} \leq\left(\hat{c}+\hat{c}_{1}\right)\left|f^{\prime}(v)\right|_{\tau}^{-l} r^{-n}
$$

Consequently the function $\lambda \mapsto N_{v, n}\left(\lambda-\lambda^{0}\right)^{n}, \lambda \in D\left(\lambda^{0}, r / 2\right)$, is analytic and ||$N_{v, n}\left(\lambda-\lambda^{0}\right)^{n} \|_{\beta} \leq\left(\hat{c}+\hat{c}_{1}\right)\left|f^{\prime}(v)\right|_{\tau}^{-l} 2^{-n}$. Thus the series

$$
A_{\lambda, v}=\sum_{n=0}^{\infty} N_{v, n}\left(\lambda-\lambda^{0}\right)^{n}, \lambda \in D\left(\lambda^{0}, r / 2\right),
$$

converges absolutely uniformly in the Banach space $L\left(\mathrm{H}_{\beta}, \mathrm{H}_{\beta, \xi}\right)$,

$$
\left\|A_{\lambda, v}\right\|_{\beta} \leq 2\left(\hat{c}+\hat{c}_{1}\right)\left|f^{\prime}(v)\right|_{\tau}^{-l}
$$

and the function $\lambda \mapsto A_{\lambda, v} \in L\left(\mathrm{H}_{\beta}, \mathrm{H}_{\beta, \xi}\right), \lambda \in D\left(\lambda^{0}, r / 2\right)$, is analytic. Note that

$$
A_{\lambda, v} g=\exp \left(\phi_{\lambda} \circ f_{v}^{-1}\right) g \circ f_{v}^{-1} .
$$

Since by (d), $l>\rho / \alpha$, it follows from (7.7) that the series

$$
\mathcal{L}_{\lambda, \xi}=\sum_{v \in f^{-1}(\xi)} A_{\lambda, v}, \lambda \in D\left(\lambda^{0}, r / 2\right),
$$


converges absolutely uniformly in the Banach space $L\left(\mathrm{H}_{\beta}, \mathrm{H}_{\beta, \xi}\right)$,

$$
\left\|\mathcal{L}_{\lambda, \xi}\right\|_{\beta} \leq 2\left(\hat{c}+\hat{c}_{1}\right) \sum_{v \in f^{-1}(\xi)}\left|f^{\prime}(v)\right|_{\tau}^{-l} \leq 2\left(\hat{c}+\hat{c}_{1}\right) M_{\hat{\tau} l}
$$

and the function $\lambda \mapsto \mathcal{L}_{\lambda, \xi}, \lambda \in D\left(\lambda^{0}, r / 2\right)$, is analytic. Since $\mathcal{L}_{\lambda, \xi}=\left(\mathcal{L}_{\lambda}\right)_{\xi}$, invoking Lemma 7.1 concludes the proof.

\subsection{Analyticity of pressure}

In this section we consider a special (affine) family of potentials and we apply Theorem 7.2, Let

$$
\phi=-t_{1} \log \left|f^{\prime}\right|_{\tau}+h_{1}: \mathcal{J}(f) \rightarrow \mathbb{R} \text { and } \psi=-t_{2} \log \left|f^{\prime}\right|_{\tau}+h_{2}: \mathcal{J}(f) \rightarrow \mathbb{R}
$$

be two arbitrary loosely tame functions. Consider the set

$$
\Sigma_{1}(\phi, \psi):=\left\{q \in \mathbb{C}: \operatorname{Re}(q) t_{1}+t_{2}>\rho / \hat{\tau}\right\} .
$$

The key ingredient (following from Theorem 7.2) to all further analytic properties of "thermodynamical objects" appearing in this section is the following.

Proposition 7.3. If $\phi, \psi: \mathcal{J}(f) \rightarrow \mathbb{R}$ are two arbitrary tame functions, then the function $q \mapsto \mathcal{L}_{q \phi+\psi}, q \in \Sigma_{1}(\phi, \psi)$, is holomorphic.

Proof. Fixing $q_{0} \in \Sigma_{1}(\phi, \psi)$ and taking $r>0$ so small that $G=D\left(q_{0}, r\right) \subset$ $\bar{D}\left(q_{0}, r\right) \subset \Sigma_{1}(\phi, \psi)$, we see that all the assumptions of Theorem 7.2 are straightforwardly satisfied. Thus, invoking this theorem, we are done.

Let us now derive some consequences of this proposition. We start with the following easy but useful fact resulting immediately from Hölder's inequality.

Lemma 7.4. If $\phi$ and $\psi$ are arbitrary tame functions, then the function $q \mapsto$ $\mathrm{P}(q \phi+\psi), q \in \Sigma_{1}(\phi, \psi) \cap \mathbb{R}$, is convex.

For every $q \in \Sigma_{1}(\phi, \psi) \cap \mathbb{R}$ let $Q_{1, q}: \mathrm{H}_{\beta} \rightarrow \mathrm{H}_{\beta}$ be the projection operator associated to the operator $\hat{\mathcal{L}}_{q \phi+\psi}$ via Theorem 6.5. Let

$$
S_{q}=\hat{\mathcal{L}}_{q \phi+\psi}-Q_{1, q}
$$

be the difference operator appearing in Theorem 6.5 and let

$$
\rho_{q}=\rho_{q \phi+\psi}
$$

be the eigenfunction of $\mathcal{L}_{q \phi+\psi}$ (fixed point of $\hat{\mathcal{L}}_{q \phi+\psi}$ ) also appearing in Theorem6.5. Using heavily Theorem $\mathbf{7 . 3}$ and the perturbation theory for linear operators (see Ka for its account), we shall prove the following.

Lemma 7.5. If $\phi$ and $\psi$ are arbitrary loosely tame functions, then all the four functions $q \mapsto \mathrm{P}(q \phi+\psi), Q_{1, q}, S_{q}, \rho_{q}, \quad q \in \Sigma_{1}(\phi, \psi) \cap \mathbb{R}$, are real-analytic. 
Proof. Fix $q_{0} \in \Sigma_{1}(\phi, \psi) \cap \mathbb{R}$. Applying now Proposition 7.3, the perturbation theory for linear operators (see $\mathbf{K a}$ ) and Theorem [6.5, we see that there exist $R_{1}>0$ (so small that $\left.D\left(q_{0}, R_{1}\right) \subset \Sigma_{1}(\phi, \psi)\right)$ and three holomorphic functions $\gamma: D\left(q_{0}, R_{1}\right) \rightarrow \mathbb{C}, Q: D\left(q_{0}, R_{1}\right) \rightarrow L\left(\mathrm{H}_{\beta}\right)$ and $\rho: D\left(q_{0}, R_{1}\right) \rightarrow \mathrm{H}_{\beta}$ such that $\gamma\left(q_{0}\right)=e^{\mathrm{P}\left(q_{0} \phi+\psi\right)}$ and $\rho\left(q_{0}\right)=\rho_{q_{0} \phi+\psi}$, for every $q \in D\left(q_{0}, R_{1}\right)$ the number $\gamma(q)$ is a simple isolated eigenvalue of the operator $\mathcal{L}_{q \phi+\psi}$ with the remainder part of the spectrum uniformly separated from $\gamma(q), \rho(q)$ is its normalized eigenfunnction, and $Q(q): \mathrm{H}_{\beta} \rightarrow \mathrm{H}_{\beta}$ is the projection operator corresponding to the eigenvalue $\gamma(q)$. In particular there exist $0<R_{2} \leq R_{1}$ and $\eta>0$ such that

$$
\Sigma_{1}\left(\mathcal{L}_{q \phi+\psi}\right) \cap D\left(\exp \left(\mathrm{P}\left(q_{0} \phi+\psi\right)\right), \eta\right)=\{\gamma(q)\}
$$

for all $q \in D\left(q_{0}, R_{2}\right)$. In view of Lemma 7.4 there thus exists $R_{3} \in\left(0, R_{2}\right]$ such that $\mathrm{P}(q \phi+\psi)=\log (\gamma(q))$ for all $D_{\mathbb{R}}\left(q_{0}, R_{3}\right)$. Consequently also $Q_{1, q}=\exp (-(\mathrm{P}(q \phi+$ $\psi)) Q(q)$ for all $q \in D_{\mathbb{R}}\left(q_{0}, R_{3}\right)$ and $g(q)=\rho_{q \phi+\psi}$. The proof is now completed by noting that $S_{q}=\exp \left(-(\mathrm{P}(q \phi+\psi)) \mathcal{L}_{q \phi+\psi}-Q_{1, q}\right.$.

Put

$$
\Sigma_{2}(\phi, \psi)=\left\{(q, t) \in \mathbb{C} \times \mathbb{C}: \operatorname{Re}(q) t_{1}+\operatorname{Re}(t) t_{2}>\rho / \hat{\tau}\right\} .
$$

We will also need the following, strictly speaking stronger, result.

Lemma 7.6. If $\phi$ and $\psi$ are two arbitrary tame functions, then all the four functions $(q, t) \mapsto \mathrm{P}(q \phi+t \psi), Q_{1,(q, t)}, S_{q, t}, \rho_{q, t}$, where $(q, t) \in \Sigma_{2}(\phi, \psi)$, (the objects $Q_{1,(q, t)}, S_{q, t}, \rho_{q, t}$ have obvious meaning) are real-analytic.

Proof. The proof goes with obvious modifications exactly as the proof of Lemma 7.5

Lemma 7.7. For every $q_{0} \in \Sigma_{1}(\phi, \psi) \cap \mathbb{R}$ there exist $\eta>0, C>0$ and $\theta \in(0,1)$ such that $\bar{D}\left(q_{0}, \eta\right) \subset \Sigma_{1}(\phi, \psi)$,

$$
\left\|S_{q}^{n}\right\|_{\alpha} \leq C \theta^{n},\left\|\hat{\mathcal{L}}_{q \phi+\psi}^{n}\right\|_{\alpha} \leq C \text { and }\left\|\rho_{q \phi+\psi}\right\|_{\alpha} \leq C .
$$

for all $q \in D_{\mathbb{R}}\left(q_{0}, \eta\right)$ and all $n \geq 0$.

Proof. It follows from Theorem 6.5(b) that there exists $u \geq 1$ such that $\left\|S_{q_{0}}^{u}\right\|_{\alpha} \leq 1 / 8$. Hence, in view of Lemma 7.5, there exists $\eta>0$ so small that $\bar{D}_{\mathbb{R}}\left(q_{0}, \eta\right) \subset \Sigma_{1}(\phi, \psi) \cap \mathbb{R}$ and $\left\|S_{q}^{u}\right\|_{\alpha} \leq 1 / 4$ for all $q \in D_{\mathbb{R}}\left(q_{0}, \eta\right)$. Using again Lemma 7.5 we see that $\left\|S_{q}\right\|_{\alpha} \leq M$ for all $q \in D_{\mathbb{R}}\left(q_{0}, \eta\right)$ and some $M \geq 1$. Hence $\left\|S_{q}^{j}\right\|_{\alpha} \leq M^{u}$ for all $q \in D_{\mathbb{R}}\left(q_{0}, \eta\right)$ all $j=0,1, \ldots, u-1$. A straightforward induction shows now that there exists a constant $C_{1}>0$ such that $\left\|S_{q}^{n}\right\|_{\alpha} \leq C_{1}(1 / 2)^{n / u}$ for all $n \geq 0$. Taking $\eta>0$ sufficiently small, it follows immediately from Lemma 7.5 that $\left\|Q_{1, q}\right\|_{\alpha} \leq C_{2}$ for some $C_{2}>0$ and all $q \in D\left(q_{0}, \eta\right)$. Hence $\left\|\hat{\mathcal{L}}_{q \phi+\psi}^{n}\right\|_{\alpha} \leq$ $\left\|Q_{1, q}\right\|_{\alpha}+\left\|S_{q}^{n}\right\|_{\alpha} \leq C_{2}+C_{1}$. Taking $C=C_{1}+C_{2}$, we are therefore done.

Now we shall prove the following strenghtening of Theorem 6.16,

Corollary 7.8. Fix $q_{0} \in \Sigma_{1}(\phi, \psi) \cap \mathbb{R}$ and let $\eta, \theta$ and $C$ come from Lemma 7.7 If $u \in \mathrm{H}_{\beta}, q \in\left(q_{0}-\delta, q_{0}+\delta\right)$, and $v \in L_{m_{q \phi+\psi}}^{1}$, then for all $n \geq 0$

$$
C_{q, n}(u, v) \leq 2 C^{2}(1+C) \theta^{n}\|u\|_{\alpha}\|v\|_{L_{m_{q \phi+\psi}^{1}}^{1}},
$$


where $C_{q, n}$ is the corellation function with respect to the measure $\mu_{q \phi+\psi}$.

Proof. Write $\mu_{q}=\mu_{q \phi+\psi}, m_{q}=m_{q \phi+\psi}, \rho_{q}=\rho_{q \phi+\psi}, U=u-\mu_{q}(u)=$ $u-\int \rho_{q} u d m_{q}$ and $V=v-\mu_{q}(v)=v-\int \rho_{q} v d m_{q}$. Using then Theorem 6.5 and Lemma [7.7 we obtain for every $n \geq 0$ that

$$
\begin{aligned}
C_{q, n}(u, v) & =\left|\int U \cdot\left(V \circ f^{n}\right) d \mu_{q}\right|=\left|\int U \rho_{q} \cdot\left(V \circ f^{n}\right) d m_{q}\right| \\
& =\left|\hat{\mathcal{L}}_{q}^{n}\left(U \rho_{q} \cdot\left(V \circ f^{n}\right)\right) d m_{q}\right|=\left|\int V \cdot \mathcal{L}_{q}^{n}\left(U \rho_{q}\right) d m_{q}\right| \\
& =\left|\int V S_{q}^{n}\left(U \rho_{q}\right) d m_{q}\right| \leq \int|V|\left|S_{q}^{n}\left(U \rho_{q}\right)\right| d m_{q} \\
& \leq\left\|S_{q}^{n}\left(U \rho_{q}\right)\right\|_{\infty} \int|V| d m_{q} \leq\left\|S_{q}^{n}\left(U \rho_{q}\right)\right\|_{\alpha}\|V\|_{L_{m_{q}}^{1}} \\
& \leq\left\|S_{q}^{n} \mid\right\|_{\alpha}\left\|U \rho_{q}\right\|_{\alpha}\left(1+\left\|\rho_{q}\right\|_{\infty}\right)\|v\|_{L_{m_{q}}^{1}} \\
& \leq C \theta^{n} 2\|u\|\left\|_{a}\right\| \rho_{q} \mid\left\|_{a}(1+C)\right\| v \|_{L_{m_{q}}^{1}} \\
& \leq 2 C^{2}(1+C) \theta^{n}\|u \mid\|\left\|_{a}\right\| v \|_{L_{m_{q}}^{1}}
\end{aligned}
$$

We are done.

\subsection{Derivatives of the Pressure function}

In this section we derive the formulas for the first and second derivatives of the pressure function. Throughout the entire section $\phi: \mathcal{J}(f) \rightarrow \mathbb{R}$, a tame function, and $\psi: \mathcal{J}(f) \rightarrow \mathbb{R}$, a loosely tame function, with some Hölder exponent $\beta \in(0,1]$, are fixed. All other considered loosely tame functions are also supposed to have Hölder exponent $\beta$. For every loosely tame function $\zeta=-t \log \left|f^{\prime}\right|_{\tau}+h$ write

$$
t=\tilde{\zeta} \text { and } h=\zeta_{0}
$$

In the proofs of Lemma 7.13 and Theorem 7.15 we will frequently need to estimate the norms of the functions $\hat{\mathcal{L}}_{\phi}^{n}(\zeta)$, where $n \geq 1$ and $\zeta$ is a loosely tame function. We would like to apply Lemma 7.7. however although $\zeta$ is Hölder continuous, it usually need not be bounded. To remedy this difficulty we notice in Lemma 7.9 below that $\hat{\mathcal{L}}_{\phi}(\zeta)$ is bounded (so belongs to $\left.\mathrm{H}_{\beta}\right)$. Writing then $\hat{\mathcal{L}}_{\phi}^{n}(\zeta)$ as $\hat{\mathcal{L}}_{\phi}^{n-1}\left(\hat{\mathcal{L}}_{\phi}(\zeta)\right.$ ), we may take fruits of Lemma 7.7. In fact Lemma 7.9 is somewhat stronger (arbitrary $G \in \mathrm{H}_{\beta}$ instead of $G=\mathbb{1}$ ) and this stronger form will be needed in the proof of Theorem 7.15. We start with the following.

Lemma 7.9. Suppose that $\phi: \mathcal{J}(f) \rightarrow \mathbb{R}$ is a tame function, $\psi: \mathcal{J}(f) \rightarrow \mathbb{R}$ is a loosely tame function, and that $\zeta: \mathcal{J}(f) \rightarrow \mathbb{R}$ is also a loosely tame function. Then there exists $\eta>0$ and $\Gamma(\zeta)>0$ such that if $|t|<\eta$ and $G \in \mathrm{H}_{\beta}$, then $\hat{\mathcal{L}}_{\phi+t \psi}(\zeta G) \in \mathrm{H}_{\beta}$ and moreover $\left\|\hat{\mathcal{L}}_{\phi+t \psi}(\zeta G)\right\|_{\beta} \leq \Gamma(\zeta)\|G\|_{\beta}$. In addition $\Gamma(\mathbb{1}) \leq C$ and $\Gamma(a \zeta+b \omega) \leq|a| \Gamma(\zeta)+|b| \Gamma(\omega)$ for all $a, b \in \mathbb{R}$ and all loosely tame functions $\omega$. 
Proof. Take $\eta \in(0,1]$ so small that $l:=\tilde{\phi}-\eta|\tilde{\psi}|>\rho / \hat{\tau}$. Put $l_{+}=\tilde{\phi}+\eta|\tilde{\psi}|$. Then for every $t \in(-\eta, \eta)$ and every $w \in \mathcal{J}(f)$ we have

$$
(-\tilde{\phi}-t \psi) \log \left|f^{\prime}(w)\right|_{\tau} \leq\left\{\begin{array}{ll}
-l \log \left|f^{\prime}(w)\right|_{\tau} & \text { if }\left|f^{\prime}(w)\right|_{\tau} \geq 1 \\
-l_{+} \log \left|f^{\prime}(w)\right|_{\tau} & \text { if }\left|f^{\prime}(w)\right|_{\tau} \leq 1
\end{array} \leq A-l \log \left|f^{\prime}(w)\right|_{\tau}\right.
$$

with some universal constant $A \geq 0$ large enough. Hence

$$
\exp \left((-\tilde{\phi}-t \psi) \log \left|f^{\prime}(w)\right|_{\tau}\right) \leq e^{A}\left|f^{\prime}(w)\right|_{\tau}^{-l}
$$

Put $B=\left\|\phi_{0}\right\|_{\infty}+\eta\left\|\psi_{0}\right\|_{\infty}$. Fix $t \in(-\eta, \eta)$ and put $\hat{\mathcal{L}}_{t}=\hat{\mathcal{L}}_{\phi+t \psi}$. We may assume without loss of generality that $\mathrm{P}(\phi+t \psi)=0$. Consider $G \in \mathrm{H}_{\beta}$. Fix now $u>0$ so small that $l-u>\rho / \hat{\tau}$. There then exists $C>0$ so large that $\left\|\zeta_{0}\right\|_{\infty}+\left.\left.|\tilde{\zeta}||\log | f^{\prime}(w)\right|_{\tau}|\leq C| f^{\prime}(w)\right|_{\tau} ^{u}$ for all $w \in \mathcal{J}(f)$. Hence

$$
|\zeta(w)| \leq C\left|f^{\prime}(w)\right|_{\tau}^{u}
$$

for all $w \in \mathcal{J}(f)$. Thus, using (17.9), for all $z \in \mathcal{J}(f)$ we have that

$$
\begin{aligned}
\left|\hat{\mathcal{L}}_{t}(\zeta G)(z)\right| & \leq \sum_{y \in f^{-1}(z)} \exp (\phi(y)+t \psi(y))|\zeta(y) \| G(y)| \\
& \leq e^{B} \sum_{y \in f^{-1}(z)} \exp \left((-\tilde{\phi}-t \tilde{\psi}) \log \left|f^{\prime}(y)\right|_{\tau}\right)\left|f^{\prime}(y)\right|_{\tau}^{u}\|G\|_{\infty} \\
& \leq C e^{A+B}\|G\|_{\infty} \sum_{y \in f^{-1}(z)}\left|f^{\prime}(y)\right|_{\tau}^{-(l-u)} \\
& \leq C e^{A+B} M_{l-u}\|G\|_{\beta},
\end{aligned}
$$

where $M_{l-u}$ has been defined just after formula (5.5). Hence

$$
\left\|\hat{\mathcal{L}}_{t}(\zeta G)\right\|_{\infty} \leq C e^{A+B} M_{l-u}\|G\|_{\beta}
$$

By Lemma[5.3 $T=\sup \left\{c_{\phi}+t \psi:|t| \leq \eta\right\}<+\infty$. Now fix $x \in \mathcal{J}(f)$ and $y \in D(x, \delta)$. Write $f^{-1}(x)=\left\{x_{k}\right\}_{k=1}^{\infty}$ and $y_{k}=f_{x_{k}}^{-1}(y), k \geq 1$. We then have for all $k \geq 1$ that

$$
\begin{aligned}
\left|\zeta\left(y_{k}\right) G\left(y_{k}\right)-\zeta\left(x_{k}\right) G\left(x_{k}\right)\right| & \leq\left|\zeta\left(x_{k}\right)\right| \cdot\left|G\left(x_{k}\right)-G\left(y_{k}\right)\right|+\left|G\left(y_{k}\right)\right| \cdot\left|\zeta\left(x_{k}\right)-\zeta\left(y_{k}\right)\right| \\
& \leq\left|\zeta\left(x_{k}\right)\right| \Delta|| G||_{\beta}|y-x|^{\beta}+|| G \|_{\infty} V_{b}(\zeta)|y-x|^{\beta} \\
& \leq|| G\left|\|_{\beta}\left(\Delta\left|\zeta\left(x_{k}\right)\right|+V_{b}(\zeta)\right)\right| y-\left.x\right|^{\beta} \\
& \leq C\|G\|_{\beta} \mid\left(\left.f^{\prime}\left(x_{k}\right)\right|_{\tau} ^{u}|y-x|^{\beta}\right.
\end{aligned}
$$

where the constant $C>0$ is so large that (17.10) remains true with $|\zeta(w)|$ replaced by $\Delta|\zeta(w)|+V_{b}(\zeta)$ and $\Delta$ comes from Lemma 4.10. By Lemma $5.3 T \sup \left\{c_{\phi+t \psi}\right.$ : 
$|t| \leq \eta\}<\infty$. Now, using this lemma, Lemma 4.9, and (7.10) we get

$$
\begin{aligned}
\left|\hat{\mathcal{L}}_{t}(\zeta G)(y)-\hat{\mathcal{L}}_{t}(\zeta G)(x)\right|= \\
=\left\|\sum_{k=1}^{\infty} \exp \left(\phi\left(y_{k}\right)+t \psi\left(y_{k}\right)\right) \zeta\left(y_{k}\right) G\left(y_{k}\right)-\exp \left(\phi\left(x_{k}\right)+t \psi\left(x_{k}\right)\right) \zeta\left(x_{k}\right) G\left(x_{k}\right) \mid\right\| \\
\leq\left\|\sum_{k=1}^{\infty}\left|\zeta\left(y_{k}\right)\right|\left|G\left(y_{k}\right)\right|\right\| \exp \left(\phi\left(y_{k}\right)+t \psi\left(y_{k}\right)\right)-\exp \left(\phi\left(x_{k}\right)+t \psi\left(x_{k}\right)\right) \mid+ \\
\quad+\sum_{k=1}^{\infty} \exp \left(\phi\left(x_{k}\right)+t \psi\left(x_{k}\right)\right)\left|\zeta\left(y_{k}\right) G\left(y_{k}\right)-\zeta\left(x_{k}\right) G\left(x_{k}\right)\right| \\
\leq C T|| G \|_{\infty} \sum_{k=1}^{\infty} \mid\left(\left.f^{\prime}\left(y_{k}\right)\right|_{\tau} ^{u} \exp \left(\phi\left(x_{k}\right)+t \psi\left(x_{k}\right)\right)|y-x|^{\beta}+\right. \\
\quad+C|| G \|_{\beta} \sum_{k=1}^{\infty} \mid\left(\left.f^{\prime}\left(x_{k}\right)\right|_{\tau} ^{u} \exp \left(\phi\left(x_{k}\right)+t \psi\left(x_{k}\right)\right)|y-x|^{\beta}\right. \\
\leq C\left(T K_{\tau}^{u}+1\right)\|G\|_{\beta}|y-x|^{\beta} \sum_{k=1}^{\infty} \mid\left(\left.f^{\prime}\left(x_{k}\right)\right|_{\tau} ^{u} \exp \left(\phi\left(x_{k}\right)+t \psi\left(x_{k}\right)\right)\right. \\
\leq C e^{A+B}\left(T K_{\tau}^{u}+1\right)\|G\|_{\beta}|y-x|^{\beta} \sum_{k=1}^{\infty} \mid\left(\left.f^{\prime}\left(x_{k}\right)\right|_{\tau} ^{-(l-u)}\right. \\
\leq C e^{A+B} M_{l-u}\left(T K_{\tau}^{u}+1\right)\|G\|_{\beta}|y-x|^{\beta},
\end{aligned}
$$

where the second last inequality was written due to (7.9) and the definition of $B$, and the justification for the last inequality is the same as that for the last line in (7.11). Hence, we obtained that $v_{\beta}\left(\hat{\mathcal{L}}_{t}(\zeta G)\right) \leq C e^{A+B} M_{l-u}\left(T K_{\tau}^{u}+1\right)\|G\|_{\beta}$. Combining this and (17.12), the proof of the first part of our lemma is complete. The second part follows immediately from Lemma 7.7. The last part is an immediate consequence of linearity of the operator $\hat{\mathcal{L}}_{t}$.

Remark 7.10. Notice that if $\zeta_{1}$ and $\zeta_{2}$ are two $\beta$-Hölder functions, then for all $x, y \in \mathcal{J}(f)$ with $|y-x| \leq \delta$ and $x_{k}, y_{k}$ as in the proof of Lemma 7.9, we have

$$
\begin{aligned}
\left|\zeta_{1} \zeta_{2}\left(y_{k}\right)-\zeta_{1} \zeta_{2}\left(x_{k}\right)\right| & =\left|\zeta_{1}\left(y_{k}\right)\left(\zeta_{2}\left(y_{k}\right)-\zeta_{2}\left(x_{k}\right)\right)+\zeta_{2}\left(x_{k}\right)\left(\zeta_{1}\left(y_{k}\right)-\zeta_{1}\left(x_{k}\right)\right)\right| \\
& \leq\left|\zeta_{1}\left(y_{k}\right)\right|\left|\zeta_{2}\left(y_{k}\right)-\zeta_{2}\left(x_{k}\right)\right|+\left|\zeta_{2}\left(x_{k}\right)\right|\left|\zeta_{1}\left(y_{k}\right)-\zeta_{1}\left(x_{k}\right)\right| \\
& \leq\left|\zeta_{1}\left(y_{k}\right)\right| V_{\beta}\left(\zeta_{2}\right)|y-x|^{\beta}+\left|\zeta_{2}\left(x_{k}\right)\right| V_{\beta}\left(\zeta_{1}\right)|y-x|^{\beta} \\
& \leq 2 \Delta \max \left\{V_{\beta}\left(\zeta_{1}\right), V_{\beta}\left(\zeta_{2}\right)\right\} \max \left\{\left|\zeta_{2}\left(x_{k}\right)\right|,\left|\zeta_{1}\left(y_{k}\right)\right|\right\}|y-x|^{\beta}
\end{aligned}
$$

Therefore, the proof of Lemma 7.9 goes through with obvious modifications with $\zeta$ replaced by the product of any two tame functions.

Before we formulate the next lemma, observe that the absolute value of a loosely tame function is is also loosely tame (in fact if $\rho$ is loosely tame, then $|\tilde{\rho}|=|\tilde{\rho}|$ ), and consequently, the absolute value of the product of two loosely tame functions is a product of two loosely tame functions. 
Lemma 7.11. Assume that $\rho$ is either a loosely tame function or a product of two loosely tame functions. With the assumptions and notation as in Lemma 7.9, we have that

for all $t \in(-\eta, \eta)$.

$$
\int|\rho| d m_{\phi+t \psi} \leq \Gamma(|\rho|)
$$

Proof. Indeed, in view Lemma 7.9 and Remark 7.10 , we have

$\int|\rho| d m_{\phi+t \psi}=\int \hat{\mathcal{L}}_{\phi+t \psi}(|\rho|) d m_{\phi+t \psi} \leq\left\|\hat{\mathcal{L}}_{\phi+t \psi}(|\rho|)\right\|_{\infty} \leq\left\|\hat{\mathcal{L}}_{\phi+t \psi}(|\rho|)\right\|_{\beta} \leq \Gamma(|\rho|)$.

We are done.

We are now able to establish the following strengthening of Theorem 6.16.

Lemma 7.12. Fix $t_{0} \in \Sigma_{1}(\psi, \phi)$ and let $\eta, \theta$, and $C$ come from Lemma 7.7. If each function $\zeta, \rho$ is either a loosely tame function or a product of two loosely tame functions and if $s \in(-\eta, \eta)$, then for all $n \geq 1$,

$$
C_{s, n}(\zeta, \rho) \leq C^{2}(1+C) \Gamma(|\rho|)\left(\Gamma(\zeta)+C^{2} \Gamma(|\zeta|)\right) \theta^{n-1},
$$

where $C_{s, n}$ is the corellation function with respect to the measure $\mu_{\phi+s \psi}$.

Proof. We use the obvious notation $\mu_{s}, m_{s}$, and $\rho_{s}$. We put $\bar{\zeta}=\zeta-\mu_{s}(\zeta)$ and $\bar{\rho}=\rho-\mu_{s}(\rho)$. Notice that by Lemma 7.11 and Lemma 7.7 we have

$$
\begin{aligned}
\int|\bar{\rho}| d m_{s} & \leq \int\left(|\rho|+\left|\mu_{s}(\rho \mid) d m_{s} \leq \int\right| \rho\left|d m_{s}+\int\right| \rho\left|\rho_{s} d m_{s} \leq \Gamma(|\rho|)+C \int\right| \rho \mid d m_{s}\right. \\
& \leq(1+C) \Gamma(|\rho|) .
\end{aligned}
$$

In view of Lemma 7.9 , the estimate $\left|\mu_{s}(\zeta)\right| \leq C \Gamma(|\zeta|)$ obtained in the computation of the previous formula, gives

$$
\Gamma(\bar{\zeta}) \leq \Gamma(\zeta)+\left|\mu_{s}(\zeta)\right| \Gamma(\mathbb{1}) \leq \Gamma(\zeta)+C^{2} \Gamma(|\zeta|) .
$$

Proceeding now exactly as in the proof of Corollary 7.8, utilising (7.13), Lemma 7.7 Lemma 7.9, Remark 7.10, and (7.14), we get

$$
\begin{aligned}
C_{s, n}(\zeta, \rho) & =\left|\int \bar{\rho} \hat{\mathcal{L}}_{s}^{n}\left(\bar{\zeta} \rho_{s}\right) d m_{s}\right|=\left|\int \bar{\rho} \hat{\mathcal{L}}_{s}^{n-1}\left(\hat{\mathcal{L}}_{s}\left(\bar{\zeta} \rho_{s}\right)\right) d m_{s}\right| \\
& =\left|\int \bar{\rho} S_{s}^{n-1}\left(\hat{\mathcal{L}}_{s}\left(\bar{\zeta} \rho_{s}\right)\right) d m_{s}\right| \leq \int|\bar{\rho}| \mid S_{s}^{n-1}\left(\hat{\mathcal{L}}_{s}\left(\bar{\zeta} \rho_{s}\right)\right) \|_{\beta} d m_{s} \\
& =\left\|S_{s}^{n-1}\left(\hat{\mathcal{L}}_{s}\left(\bar{\zeta} \rho_{s}\right)\right)\right\|_{\beta} \int|\bar{\rho}| d m_{s} \leq\left\|S_{s}^{n-1}\right\|_{\beta}\left\|\hat{\mathcal{L}}_{s}\left(\bar{\zeta} \rho_{s}\right)\right\|_{\beta}(1+C) \Gamma(|\rho|) \\
& \leq C(1+C) \Gamma(|\rho|) \theta^{n-1}\left\|\hat{\mathcal{L}}_{s}\left(\bar{\zeta} \rho_{s}\right)\right\|_{\beta} \leq C(1+C) \Gamma(|\rho|) \theta^{n-1} \Gamma(\bar{\zeta})\left\|\rho_{s}\right\|_{\beta} \\
& \leq C^{2}(1+C) \Gamma(|\rho|)\left(\Gamma(\zeta)+C^{2} \Gamma(|\zeta|)\right) \theta^{n-1} .
\end{aligned}
$$

We are done.

The next lemma is the key point in the proof of Theorem 7.14, providing a formula for the derivative of the pressure function. 
Lemma 7.13. Assume the same as in Lemma 7.9, Fix $x \in \mathcal{J}(f)$. Then

$$
\lim _{n \rightarrow \infty} \frac{1}{n} \frac{\sum_{y \in f^{-n}(x)} S_{n} \zeta(y) \exp \left(S_{n}(\phi+t \psi)(y)\right)}{\sum_{y \in f^{-n}(x)} \exp \left(S_{n}(\phi+t \psi)(y)\right)}=\int \zeta d \mu_{\phi+t \psi}
$$

uniformly with respect to all $t \in(-\eta, \eta)$, where $\eta$ comes from Lemma 7.9 .

Proof. Put for every $t \in \mathbb{R}$,

$$
\mathcal{L}_{t}=\mathcal{L}_{\phi+t \psi} \text { and } \hat{\mathcal{L}}_{t}=\hat{\mathcal{L}}_{\phi+t \psi}
$$

and

$$
g_{t}=g_{\phi+t \psi}=Q_{1, t}(\mathbb{1})
$$

First observe that $n$th term of the sequence from our lemma is equal to (7.15)

$$
\frac{1}{n} \sum_{j=0}^{n-1} \frac{\hat{\mathcal{L}}_{t}^{n}\left(\zeta \circ f^{j}\right)(x)}{\hat{\mathcal{L}}_{t}^{n}(\mathbb{1})(x)}=\frac{1}{n} \sum_{j=0}^{n-1} \frac{\hat{\mathcal{L}}_{t}^{n-j}\left(\zeta \hat{\mathcal{L}}_{t}^{j}(\mathbb{1})\right)(x)}{\hat{\mathcal{L}}_{t}^{n}(\mathbb{1})(x)}=\frac{1}{n} \sum_{j=0}^{n-1} \frac{\hat{\mathcal{L}}_{t}^{n-j-1}\left(\hat{\mathcal{L}}_{t}\left(\zeta \hat{\mathcal{L}}_{t}^{j}(\mathbb{1})\right)\right)(x)}{\hat{\mathcal{L}}_{t}^{n}(\mathbb{1})(x)} .
$$

We now look at the difference $\frac{1}{n} \sum_{j=0}^{n-1} \frac{\hat{\mathcal{L}}_{t}^{n}\left(\zeta \circ F^{j}\right)(x)}{\hat{\mathcal{L}}_{t}^{n}(\mathbb{1})(x)}-\int \zeta d \mu_{\phi+t \psi}$ and split it into three terms each of which will turn out to converge (uniformly in $t$ ) to zero because of Lemma 7.7 and Lemma 7.9. Indeed (note that $Q_{1, t}=g_{t}$ ),

$$
\begin{aligned}
& \frac{1}{n} \sum_{j=0}^{n-1} \frac{\hat{\mathcal{L}}_{t}^{n}\left(\zeta \circ F^{j}\right)(x)}{\hat{\mathcal{L}}_{t}^{n}(\mathbb{1})(x)}-\int \zeta d \mu_{\phi+t \psi}= \\
& =\frac{1}{n} \sum_{j=0}^{n-1} \frac{\hat{\mathcal{L}}_{t}^{n-j-1}\left(\hat{\mathcal{L}}_{t}\left(\zeta \hat{\mathcal{L}}_{t}^{j}(\mathbb{1})\right)\right)(x)}{g_{t}(x)}+ \\
& \quad+\frac{1}{n} \sum_{j=0}^{n-1} \frac{\hat{\mathcal{L}}_{t}^{n-j-1}\left(\hat{\mathcal{L}}_{t}\left(\zeta \hat{\mathcal{L}}_{t}^{j}(\mathbb{1})\right)\right)(x)\left(Q_{1, t}(\mathbb{1})(x)-\hat{\mathcal{L}}_{t}^{n}(\mathbb{1})(x)\right)}{\hat{\mathcal{L}}_{t}^{n}(\mathbb{1})(x) g_{t}(x)}- \\
& -\frac{1}{n} \sum_{j=0}^{n-1} \int \hat{\mathcal{L}}_{t}\left(\zeta \hat{\mathcal{L}}_{t}^{j}(\mathbb{1})\right) d m_{\phi+t \psi}+\left(\frac{1}{n} \sum_{j=0}^{n-1} \int \hat{\mathcal{L}}_{t}\left(\zeta \hat{\mathcal{L}}_{t}^{j}(\mathbb{1})\right) d m_{\phi+t \psi}-\int \zeta d \mu_{\phi+t \psi}\right) \\
& =g_{t}^{-1}(x) \frac{1}{n} \sum_{j=0}^{n-1}\left(\hat{\mathcal{L}}_{t}^{n-j-1}\left(\hat{\mathcal{L}}_{t}\left(\zeta \hat{\mathcal{L}}_{t}^{j}(\mathbb{1})\right)\right)(x)-g_{t}(x) \int \hat{\mathcal{L}}_{t}\left(\zeta \hat{\mathcal{L}}_{t}^{j}(\mathbb{1})\right) d \mu_{\phi+t \psi}\right)+ \\
& \quad+\frac{1}{n}\left(Q_{1, t}(\mathbb{1})(x)-\hat{\mathcal{L}}_{t}^{n}(\mathbb{1})(x)\right) \frac{\sum_{j=0}^{n-1} \hat{\mathcal{L}}_{t}^{n-j-1}\left(\hat{\mathcal{L}}_{t}\left(\zeta \hat{\mathcal{L}}_{t}^{j}(\mathbb{1})\right)\right)(x)}{g_{t}(x) \hat{\mathcal{L}}_{t}^{n}(\mathbb{1})(x)}+ \\
& \quad+\frac{1}{n} \sum_{j=0}^{n-1} \int \zeta \hat{\mathcal{L}}_{t}^{j}(\mathbb{1}) d m_{\phi+t \psi}-\int g_{t} \zeta d m_{\phi+t \psi}
\end{aligned}
$$




$$
\begin{aligned}
=g_{t}^{-1}(x) & \frac{1}{n} \sum_{j=0}^{n-1}\left(\hat{\mathcal{L}}_{t}^{n-j-1}\left(\hat{\mathcal{L}}_{t}\left(\zeta \hat{\mathcal{L}}_{t}^{j}(\mathbb{1})\right)\right)(x)-g_{t}(x) \int \hat{\mathcal{L}}_{t}\left(\zeta \hat{\mathcal{L}}_{t}^{j}(\mathbb{1})\right) d m_{\phi+t \psi}\right)+ \\
& +\frac{1}{n}\left(Q_{1, t}(\mathbb{1})(x)-\hat{\mathcal{L}}_{t}^{n}(\mathbb{1})(x)\right) \frac{\sum_{j=0}^{n-1} \hat{\mathcal{L}}_{t}^{n-j-1}\left(\hat{\mathcal{L}}_{t}\left(\zeta \hat{\mathcal{L}}_{t}^{j}(\mathbb{1})\right)\right)(x)}{g_{t}(x) \hat{\mathcal{L}}_{t}^{n}(\mathbb{1})(x)}+ \\
& +\frac{1}{n} \sum_{j=0}^{n-1} \int \zeta\left(\hat{\mathcal{L}}_{t}^{j}(\mathbb{1})-g_{t}\right) d m_{\phi+t \psi}
\end{aligned}
$$

It immediately follows from Lemma 7.7 and Lemma 7.9 (with $G$ being of the form $\left.\hat{\mathcal{L}}_{t}^{j}(\mathbb{1})\right)$ that the $\beta$-Hölder norm (and so the supremum norm as well) of the second summand converges to zero uniformly with respect to $t \in(-\eta, \eta)$. Dealing with the first summand notice that, in view of Lemma 7.9 and Lemma 7.7 applied twice, we get that

$$
\begin{aligned}
\| \hat{\mathcal{L}}_{t}^{n-j-1}\left(\hat{\mathcal{L}}_{t}\left(\zeta \hat{\mathcal{L}}_{t}^{j}(\mathbb{1})\right)\right) & -g_{t} \int \hat{\mathcal{L}}_{t}\left(\zeta \hat{\mathcal{L}}_{t}^{j}(\mathbb{1})\right) d m_{\phi+t \psi} \|_{\beta}= \\
& =\left\|\hat{\mathcal{L}}_{t}^{n-j-1}\left(\hat{\mathcal{L}}_{t}\left(\zeta \hat{\mathcal{L}}_{t}^{j}(\mathbb{1})\right)\right)-Q_{1, t}\left(\hat{\mathcal{L}}_{t}\left(\zeta \hat{\mathcal{L}}_{t}^{j}(\mathbb{1})\right)\right)\right\|_{\beta} \\
& =\left\|\left(\hat{\mathcal{L}}_{t}^{n-j-1}-Q_{1, t}\right)\left(\hat{\mathcal{L}}_{t}\left(\zeta \hat{\mathcal{L}}_{t}^{j}(\mathbb{1})\right)\right)\right\|_{\beta} \\
& \leq\left\|\hat{\mathcal{L}}_{t}^{n-j-1}-Q_{1, t}\right\|_{\beta}\left\|\hat{\mathcal{L}}_{t}\left(\zeta \hat{\mathcal{L}}_{t}^{j}(\mathbb{1})\right)\right\|_{\beta} \\
& \leq C \Gamma(\zeta)\left\|\hat{\mathcal{L}}_{t}^{n-j-1}-Q_{1, t}\right\|_{\beta} \\
& =C \Gamma(\zeta)\left\|S_{t}^{n-j-1}\right\|_{\beta} \\
& \leq C^{2} \Gamma(\zeta) \theta^{n-j-1}
\end{aligned}
$$

Therefore, applying Lemma 7.7 we see that the first summand in the last part of (7.16) converges to zero uniformly with respect to $t \in(-\eta, \eta)$. It follows immediately from Lemma 7.11 and Lemma 7.7 that so does the third summand in the last part of (7.16). We are done.

The first main result of this section, the formula for the first derivative of the pressure function, is this.

Theorem 7.14. Suppose that $\phi: \mathcal{J}(f) \rightarrow \mathbb{R}$ is a tame function and $\psi: \mathcal{J}(f) \rightarrow \mathbb{R}$ is a loosely tame function. Then

$$
\left.\frac{d}{d t}\right|_{t=0} \mathrm{P}(\phi+t \psi)=\int \psi d \mu_{\phi}
$$

Proof. Fix $x \in \mathcal{J}(f)$. Put

$$
\mathrm{P}_{n}(t)=\frac{1}{n} \log \sum_{y \in f^{-n}(x)} \exp \left(S_{n}(\phi+t \psi)(y)\right) .
$$

Then

$$
\frac{d \mathrm{P}_{n}}{d t}=\frac{1}{n} \frac{\sum_{y \in f^{-n}(x)} S_{n} \psi(y) \exp \left(S_{n}(\phi+t \psi)(y)\right)}{\sum_{y \in f^{-n}(x)} \exp \left(S_{n}(\phi+t \psi)(y)\right)},
$$


and, in view of Lemma 7.13. $\frac{d \mathrm{P}_{n}}{d t}$ converges uniformly with respect to $t \in(-\eta, \eta)$ to $\int \psi d \mu_{\phi+t \psi}$. Since, in addition, $\lim _{n \rightarrow \infty} \mathrm{P}_{n}(t)=\mathrm{P}(\phi+t \psi)$, we conclude that $\frac{d \mathrm{P}}{d t}=\int \psi d \mu_{\phi+t \psi}$ for all $t \in(-\eta, \eta)$. Taking $t=0$ we are therefore done.

We are now in position to prove the following second main result of this section, the formula for the second derivative of the pressure function.

Theorem 7.15. Suppose that $\phi: \mathcal{J}(f) \rightarrow \mathbb{R}$ is a tame function and $\psi, \zeta: \mathcal{J}(f) \rightarrow$ $\mathbb{R}$ are loosely tame functions. Then

$$
\left.\frac{\partial^{2}}{\partial s \partial t}\right|_{(0,0)} \mathrm{P}(\phi+s \psi+t \zeta)=\sigma^{2}(\psi, \zeta)
$$

where

$$
\begin{aligned}
& \sigma^{2}(\psi, \zeta)= \lim _{n \rightarrow \infty} \frac{1}{n} \int S_{n}\left(\psi-\mu_{\phi}(\psi)\right) S_{n}\left(\zeta-\mu_{\phi}(\zeta)\right) d \mu_{\phi} \\
&=\int\left(\psi-\mu_{\phi}(\psi)\right)\left(\zeta-\mu_{\phi}(\zeta)\right) d \mu_{\phi}+\sum_{k=1}^{\infty} \int\left(\psi-\mu_{\phi}(\psi)\right)\left(\zeta-\mu_{\phi}(\zeta)\right) \circ f^{k} d \mu_{\phi} \\
& \quad+\sum_{k=1}^{\infty} \int\left(\zeta-\mu_{\phi}(\zeta)\right)\left(\psi-\mu_{\phi}(\psi)\right) \circ f^{k} d \mu_{\phi}
\end{aligned}
$$

(if $\psi=\zeta$ we simply write $\sigma^{2}(\psi)$ for $\sigma^{2}(\psi, \psi)$ )

Proof. Put

$$
\hat{\mathcal{L}}_{s}=\hat{\mathcal{L}}_{\phi+s \psi}
$$

The symbols $m_{s}, \mu_{s}$ and $\rho_{s}$ have also the corresponding obvious meaning. It follows from the proof of Theorem 7.14 that

$$
\left.\frac{\partial^{2}}{\partial s \partial t}\right|_{t=0} \mathrm{P}(\phi+s \psi+t \zeta)=\frac{d}{d s} \lim _{n \rightarrow \infty} \frac{1}{n} \frac{\sum_{y \in f^{-n}(x)} S_{n} \zeta(y) \exp \left(S_{n}(\phi+s \psi)(y)\right)}{\sum_{y \in f^{-n}(x)} \exp \left(S_{n}(\phi+s \psi)(y)\right)}
$$

for all $s$ sufficiently small in absolute value. Fix $x \in \mathcal{J}(f), n \geq 1$ and abbreviate the notation $\sum_{y \in f^{-n}(x)}$ to $\sum_{y}$. Let

$$
\Delta_{n}(s):=\frac{d}{d s}\left(\frac{\sum_{y} S_{n} \zeta(y) \exp \left(S_{n}(\phi+s \psi)(y)\right)}{\sum_{y} \exp \left(S_{n}(\phi+s \psi)(y)\right)}\right) .
$$

The idea of the proof is to show that the sequence $\left(\frac{1}{n} \Delta_{n}(s)\right)_{1}^{\infty}$ converges to $\sigma^{2}(\psi, \zeta)$ uniformly on some sufficiently small neighbouhood of zero. In fact we will show that $\left|\Delta_{n}(s)-\int S_{n}\left(\psi-\mu_{s}(\psi)\right) S_{n}\left(\zeta-\mu_{s}(\zeta)\right) d \mu_{s}\right|$ stays uniformly bounded on such neighborhood. The proof will consist of six steps starting with measures $\mu_{s, n}$ (instead $\mu_{s}$ ) and ending with the measures $\mu_{s}$. 
Step 1: We have this.

$$
\begin{aligned}
\Delta_{n}(s)= & \frac{\sum_{y} S_{n} \psi(y) S_{n} \zeta(y) \exp \left(S_{n}(\phi+s \psi)(y)\right)}{\sum_{y} \exp \left(S_{n}(\phi+s \psi)(y)\right)}- \\
& -\frac{\left(\sum_{y} S_{n} \psi(y) \exp \left(S_{n}(\phi+s \psi)(y)\right)\right) \sum_{y} S_{n} \zeta(y) \exp \left(S_{n}(\phi+s \psi)(y)\right)}{\left(\sum_{y} \exp \left(S_{n}(\phi+s \psi)(y)\right)\right)^{2}} \\
= & \frac{\hat{\mathcal{L}}_{s}^{n}\left(S_{n} \psi S_{n} \zeta\right)(x)}{\hat{\mathcal{L}}_{s}^{n}(\mathbb{1})(x)}-\frac{\hat{\mathcal{L}}_{s}^{n} \psi(x) \hat{\mathcal{L}}_{s}^{n} \zeta(x)}{\hat{\mathcal{L}}_{s}^{n}(\mathbb{1})(x) \hat{\mathcal{L}}_{s}^{n}(\mathbb{1})(x)} \\
= & \int S_{n} \psi S_{n} \zeta d \mu_{s, n}-\int S_{n} \psi d \mu_{s, n} \int S_{n} \zeta d \mu_{s, n} \\
= & \sum_{i=0}^{n-1} \sum_{j=0}^{n-1} \int\left(\psi \circ f^{i}-\mu_{s, n}\left(\psi \circ f^{i}\right)\right)\left(\zeta \circ f^{i}-\mu_{s, n}\left(\zeta \circ f^{i}\right)\right) d \mu_{s, n},
\end{aligned}
$$

where

$$
\mu_{s, n}=\frac{\sum_{y} \delta_{y} \exp \left(S_{n}(\phi+s \psi)(y)\right)}{\sum_{y} \exp \left(S_{n}(\phi+s \psi)(y)\right)}
$$

and $\delta_{y}$ is the Dirac measure supported at $y$. In order to simplify notation put $\psi_{i}=\psi \circ f^{i}, \zeta_{j}=\zeta \circ f^{j}$ and

$$
K_{i, j}=\int\left(\psi_{i}-\mu_{s, n}\left(\psi_{i}\right)\right)\left(\zeta_{j}-\mu_{s, n}\left(\zeta_{j}\right)\right) d \mu_{s, n}=\frac{\hat{\mathcal{L}}_{s}^{n}\left(\left(\psi_{i}-\mu_{s, n}\left(\psi_{i}\right)\right)\left(\zeta_{j}-\mu_{s, n}\left(\zeta_{j}\right)\right)\right)}{\hat{\mathcal{L}}_{s}^{n}(\mathbb{1})(x)} .
$$

Fix now $0 \leq i \leq j \leq n-1$. Then

$$
K_{i, j}=\frac{\left.\hat{\mathcal{L}}_{s}^{n-j}\left(\hat{\mathcal{L}}_{s}^{j-i}\left(\left(\psi-\mu_{s, n}\left(\psi_{i}\right)\right) \hat{\mathcal{L}}_{s}^{j}(\mathbb{1})\right)\left(\zeta-\mu_{s, n}\left(\zeta_{j}\right)\right)\right)(x)\right)}{\hat{\mathcal{L}}_{s}^{n}(\mathbb{1})(x)} .
$$

Step 2: In this step we approximate $\sum_{i, j} K_{i, j}$ by the same terms with measures $m_{s}$ instead of $\mu_{s, n}$. This is eventually done in (7.24), (7.25) and (7.26). If $j>i$, it follows from Lemma 7.7 and Lemma 7.9, with $G$ being of the form $\hat{\mathcal{L}}_{s}^{i}(\mathbb{1})$, that for every $s \in(-\eta, \eta)$, we have

$$
\begin{aligned}
& \left\|\hat{\mathcal{L}}_{s}^{j-i}\left(\left(\psi-\mu_{s, n}\left(\psi_{i}\right)\right) \hat{\mathcal{L}}_{s}^{i}(\mathbb{1})\right)-\rho_{s} \int\left(\psi-\mu_{s, n}\left(\psi_{i}\right)\right) \hat{\mathcal{L}}_{s}^{i}(\mathbb{1}) d m_{s}\right\|_{\beta}= \\
& \quad=\left\|\hat{\mathcal{L}}_{s}^{j-i-1}\left(\hat{\mathcal{L}}_{s}\left(\left(\psi-\mu_{s, n}\left(\psi_{i}\right)\right) \hat{\mathcal{L}}_{s}^{j}(\mathbb{1})\right)\right)-\rho_{s} \int \hat{\mathcal{L}}_{s}\left(\left(\psi-\mu_{s, n}\left(\psi_{i}\right)\right) \hat{\mathcal{L}}_{s}^{i}(\mathbb{1})\right) d m_{s}\right\|_{\beta} \\
& \quad=\left\|S_{s}^{j-i-1}\left(\hat{\mathcal{L}}_{s}\left(\left(\psi-\mu_{s, n}\left(\psi_{i}\right)\right) \hat{\mathcal{L}}_{s}^{i}(\mathbb{1})\right)\right)\right\|_{\beta} \\
& \quad \leq C \theta^{j-i-1}|| \hat{\mathcal{L}}_{s}\left(\left(\psi-\mu_{s, n}\left(\psi_{i}\right)\right) \hat{\mathcal{L}}_{s}^{i}(\mathbb{1})\right) \|_{\beta} \\
& \quad \leq C \theta^{j-i-1}\left(\left\|\hat{\mathcal{L}}_{s}\left(\left(\psi \hat{\mathcal{L}}_{s}^{i}(\mathbb{1})\right)\left\|_{\beta}+\mu_{s, n}\left(\psi_{i}\right)\right\| \hat{\mathcal{L}}_{s}^{i+1}(\mathbb{1})\right)\right\|_{\beta}\right) \\
& \quad \leq C \theta^{j-i-1}\left(C \Gamma(\psi)+C\left|\mu_{s, n}\left(\psi_{i}\right)\right|\right)=C^{2} \theta^{j-i-1}\left(\Gamma(\psi)+\left|\mu_{s, n}\left(\psi_{i}\right)\right|\right)
\end{aligned}
$$

In view of Theorem 5.15 (4) there exists $n_{1} \geq 1$ such that for all $n \geq n_{1}, \hat{\mathcal{L}}_{s}^{n}(\mathbb{1})(x) \geq$ $\rho_{s}(x) / 2$, and in view of Lemma 7.5. $\rho_{s}(x) \geq g(x) / 2$ assuming $\eta>0$ to be small 
enough. Denote $g_{0}(x)$ by $g$. Using Lemma 7.7 and Lemma 7.9, we then get $(7.19)$

$$
\begin{aligned}
\left.\mid \mu_{s, n}\left(\psi_{i}\right)\right) \mid & =\frac{\left|\hat{\mathcal{L}}_{s}^{n} \psi_{i}(x)\right|}{\left|\hat{\mathcal{L}}_{s}^{n} \mathbb{1}(x)\right|}=\frac{\left|\hat{\mathcal{L}}_{s}^{n-i} \hat{\mathcal{L}}_{s}^{i}\left(\psi \circ f^{i}\right)(x)\right|}{\hat{\mathcal{L}}_{s}^{n} \mathbb{1}(x)}=\frac{\left|\hat{\mathcal{L}}_{s}^{n-i}\left(\psi \hat{\mathcal{L}}_{s}^{i}(\mathbb{1})\right)(x)\right|}{\hat{\mathcal{L}}_{s}^{n} \mathbb{1}(x)} \\
& =\frac{\left|\hat{\mathcal{L}}_{s}^{n-i-1}\left(\hat{\mathcal{L}}_{s}\left(\psi \hat{\mathcal{L}}_{s}^{i}(\mathbb{1})\right)\right)(x)\right|}{\hat{\mathcal{L}}_{s}^{n} \mathbb{1}(x)} \leq \frac{C \Gamma(\psi) \hat{\mathcal{L}}_{s}^{n-i-1}(\mathbb{1})(x)}{\hat{\mathcal{L}}_{s}^{n} \mathbb{1}(x)} \leq \frac{C^{2} \Gamma(\psi)}{\hat{\mathcal{L}}_{s}^{n} \mathbb{1}(x)} \\
& \leq \frac{4 C^{2} \Gamma(\psi)}{g} .
\end{aligned}
$$

It therefore follows from (7.18) that

$$
\begin{aligned}
& \| \hat{\mathcal{L}}_{s}^{j-i}\left(\left(\psi-\mu_{s, n}\left(\psi_{i}\right)\right) \hat{\mathcal{L}}_{s}^{i}(\mathbb{1})-\rho_{s} \int\right.\left(\psi-\mu_{s, n}\left(\psi_{i}\right)\right) \hat{\mathcal{L}}_{s}^{i}(\mathbb{1}) d m_{s} \|_{\beta} \\
& \leq C^{2} \Gamma(\psi)\left(1+4 C^{2} g^{-1}\right) \theta^{j-i-1}
\end{aligned}
$$

Now

$$
\begin{aligned}
& \left|\int\left(\psi-\mu_{s, n}\left(\psi_{i}\right)\right) \hat{\mathcal{L}}_{s}^{i}(\mathbb{1}) d m_{s}\right|= \\
& =\left|\int \hat{\mathcal{L}}_{s}\left(\psi \hat{\mathcal{L}}_{s}^{i}(\mathbb{1})\right) d m_{s}-\mu_{s, n}\left(\psi_{i}\right)\right| \\
& =\left|\int \hat{\mathcal{L}}_{s}\left(\psi \hat{\mathcal{L}}_{s}^{i}(\mathbb{1})\right) d m_{s}-\frac{\hat{\mathcal{L}}_{s}^{n} \psi_{i}(x)}{\hat{\mathcal{L}}_{s}^{n} \mathbb{1}(x)}\right|=\frac{\left|\hat{\mathcal{L}}_{s}^{n} \mathbb{1}(x) \int \hat{\mathcal{L}}_{s}\left(\psi \hat{\mathcal{L}}_{s}^{i}(\mathbb{1})\right) d m_{s}-\hat{\mathcal{L}}_{s}^{n} \psi_{i}(x)\right|}{\hat{\mathcal{L}}_{s}^{n} \mathbb{1}(x)} \\
& =\frac{\left|\hat{\mathcal{L}}_{s}^{n} \mathbb{1}(x) \int \hat{\mathcal{L}}_{s}\left(\psi \hat{\mathcal{L}}_{s}^{i}(\mathbb{1})\right) d m_{s}-\hat{\mathcal{L}}_{s}^{n-i} \hat{\mathcal{L}}_{s}^{i}\left(\psi_{i}\right)(x)\right|}{\hat{\mathcal{L}}_{s}^{n} \mathbb{1}(x)} \\
& =\frac{\left|\hat{\mathcal{L}}_{s}^{n} \mathbb{1}(x) \int \hat{\mathcal{L}}_{s}\left(\psi \hat{\mathcal{L}}_{s}^{i}(\mathbb{1})\right) d m_{s}-\hat{\mathcal{L}}_{s}^{n-i}\left(\psi \hat{\mathcal{L}}_{s}^{i}(\mathbb{1})\right)(x)\right|}{\hat{\mathcal{L}}_{s}^{n} \mathbb{1}(x)} \\
& =\frac{1}{\hat{\mathcal{L}}_{s}^{n} \mathbb{1}(x)}\left\{\mid\left(\hat{\mathcal{L}}_{s}^{n} \mathbb{1}(x)-\rho_{s}(x)\right) \int \hat{\mathcal{L}}_{s}\left(\psi \hat{\mathcal{L}}_{s}^{i}(\mathbb{1})\right) d m_{s}\right. \\
& \left.\quad+\left(\rho_{s}(x) \int \hat{\mathcal{L}}_{s}\left(\psi \hat{\mathcal{L}}_{s}^{i}(\mathbb{1})\right) d m_{s}-\hat{\mathcal{L}}_{s}^{n-i-1}\left(\hat{\mathcal{L}}_{s}\left(\psi \hat{\mathcal{L}}_{s}^{i}(\mathbb{1})\right)\right)(x)\right) \mid\right\}
\end{aligned}
$$

Assuming now that $n \geq n_{1}$, it therefore follows from Lemma 7.7 and Lemma 7.9 that

$$
\begin{aligned}
\left|\int\left(\psi-\mu_{s, n}\left(\psi_{i}\right)\right) \hat{\mathcal{L}}_{s}^{i}(\mathbb{1}) d m_{s}\right| \\
\quad \leq \frac{C \Gamma(\psi)\left|S_{s}^{n}(\mathbb{1})(x)\right|+\left|S_{s}^{n-i-1}\left(\hat{\mathcal{L}}_{s}\left(\psi \hat{\mathcal{L}}_{s}^{i}(\mathbb{1})\right)\right)(x)\right|}{g / 4} \\
\quad \leq \frac{C^{2} \Gamma(\psi) \theta^{n}+C \theta^{n-i-1} C \Gamma(\psi)}{g / 4} \\
\leq 8 C^{2} \Gamma(\psi) g^{-1} \theta^{n-i-1} .
\end{aligned}
$$


Assuming $\eta>0$ to be small enough, we have $\hat{g}=\sup \left\{\left\|\rho_{s}\right\|_{\infty}: s \in(-\eta, \eta)\right\}<\infty$. Combining now (7.21) and (7.20), we get for every $j=0,1, \ldots, n-1$ that

$$
\begin{aligned}
\| \sum_{i=0}^{j-1} \hat{\mathcal{L}}_{s}^{j-i} & \left(\left(\psi-\mu_{s, n}\left(\psi_{i}\right)\right) \hat{\mathcal{L}}_{s}^{i}(\mathbb{1}) \|_{\beta}\right. \\
\leq & \left(C^{2} \Gamma(\psi)\left(1+4 C^{2} g^{-1}\right)+8 C^{3} \Gamma(\psi) g^{-1}\right) \sum_{i=0}^{j-1} \theta^{j-i-1} \\
\leq & C^{2} \theta^{-1} \Gamma(\psi)\left(1+4 C^{2} g^{-1}+8 C g^{-1}\right) \sum_{i=0}^{\infty} \theta^{i}:=C_{1}<\infty .
\end{aligned}
$$

It therefore follows from Lemma 7.7, Lemma 7.9, (7.22) and (7.19) (with $\psi_{i}$ replaced by $\left.\zeta_{j}\right)$, that

$$
\begin{aligned}
& \| \hat{\mathcal{L}}_{s}^{n-j}\left(\sum_{i=0}^{j-1} \hat{\mathcal{L}}_{s}^{j-i}\left(\left(\psi-\mu_{s, n}\left(\psi_{i}\right)\right) \hat{\mathcal{L}}_{s}^{i}(\mathbb{1})\right)\left(\zeta-\mu_{s, n}\left(\zeta_{j}\right)\right)\right) \\
&- \rho_{s} \int \sum_{i=0}^{j-1} \hat{\mathcal{L}}_{s}^{j-i}\left(\left(\psi-\mu_{s, n}\left(\psi_{i}\right)\right) \hat{\mathcal{L}}_{s}^{i}(\mathbb{1})\right)\left(\zeta-\mu_{s, n}\left(\zeta_{j}\right)\right) d m_{s} \|_{\beta} \\
&=\| \hat{\mathcal{L}}_{s}^{n-j-1}\left(\hat{\mathcal{L}}_{s}\left(\sum_{i=0}^{j-1} \hat{\mathcal{L}}_{s}^{j-i}\left(\left(\psi-\mu_{s, n}\left(\psi_{i}\right)\right) \hat{\mathcal{L}}_{s}^{i}(\mathbb{1})\right)\left(\zeta-\mu_{s, n}\left(\zeta_{j}\right)\right)\right)\right)- \\
& \quad-\rho_{s} \int \hat{\mathcal{L}}_{s}\left(\sum_{i=0}^{j-1} \hat{\mathcal{L}}_{s}^{j-i}\left(\left(\psi-\mu_{s, n}\left(\psi_{i}\right)\right) \hat{\mathcal{L}}_{s}^{i}(\mathbb{1})\right)\left(\zeta-\mu_{s, n}\left(\zeta_{j}\right)\right)\right) d m_{s} \|_{\beta} \\
& \leq\left\|S_{s}^{n-j-1}\left(\hat{\mathcal{L}}_{s}\left(\sum_{i=0}^{j-1} \hat{\mathcal{L}}_{s}^{j-i}\left(\left(\psi-\mu_{s, n}\left(\psi_{i}\right)\right) \hat{\mathcal{L}}_{s}^{i}(\mathbb{1})\right)\left(\zeta-\mu_{s, n}\left(\zeta_{j}\right)\right)\right)\right)\right\|_{\beta} \\
& \leq C \theta^{n-j-1}\left\|\hat{\mathcal{L}}_{s}\left(\sum_{i=0}^{j-1} \hat{\mathcal{L}}_{s}^{j-i}\left(\left(\psi-\mu_{s, n}\left(\psi_{i}\right)\right) \hat{\mathcal{L}}_{s}^{i}(\mathbb{1})\right)\left(\zeta-\mu_{s, n}\left(\zeta_{j}\right)\right)\right)\right\|_{\beta} \\
& \leq C \theta^{n-j-1}\left(\left\|\hat{\mathcal{L}}_{s}\left(\sum_{i=0}^{j-1} \hat{\mathcal{L}}_{s}^{j-i}\left(\left(\psi-\mu_{s, n}\left(\psi_{i}\right)\right) \hat{\mathcal{L}}_{s}^{i}(\mathbb{1})\right) \zeta\right)\right\|_{\beta}+\right. \\
&\left.\quad+\left|\mu_{s, n}\left(\zeta_{j}\right)\right|\left\|\hat{\mathcal{L}}_{s}\left(\sum_{i=0}^{j-1} \hat{\mathcal{L}}_{s}^{j-i}\left(\left(\psi-\mu_{s, n}\left(\psi_{i}\right)\right) \hat{\mathcal{L}}_{s}^{i}(\mathbb{1})\right)\right)\right\|_{\beta}\right) \\
&\left.\leq C \theta^{n-j-1}\left(\Gamma(\zeta) C_{1}\right)+\left|\mu_{s, n}\left(\zeta_{j}\right)\right| C C_{1}\right) \\
& \leq C_{2} \theta^{n-j-1}, \\
&(7.23) \leq
\end{aligned}
$$


where $C_{2}=C C_{1}\left(\Gamma(\zeta)+4 C^{3} \Gamma(\zeta) g^{-1}\right)$. Now

$$
\begin{aligned}
\int \hat{\mathcal{L}}_{s}^{j-i}((\psi & \left.\left.-\mu_{s, n}\left(\psi_{i}\right)\right) \hat{\mathcal{L}}_{s}^{i}(\mathbb{1})\right)\left(\zeta-\mu_{s, n}\left(\zeta_{j}\right)\right) d m_{s}= \\
& =\int \hat{\mathcal{L}}_{s}^{n-j}\left(\hat{\mathcal{L}}_{s}^{j-i}\left(\left(\psi-\mu_{s, n}\left(\psi_{i}\right)\right) \hat{\mathcal{L}}_{s}^{i}(\mathbb{1})\right)\left(\zeta-\mu_{s, n}\left(\zeta_{j}\right)\right)\right) d m_{s} \\
& =\int \hat{\mathcal{L}}_{s}^{n}\left(\left(\psi_{i}-\mu_{s, n}\left(\psi_{i}\right)\right)\left(\zeta_{j}-\mu_{s, n}\left(\zeta_{j}\right)\right)\right) d m_{s} \\
& =\int\left(\psi_{i}-\mu_{s, n}\left(\psi_{i}\right)\right)\left(\zeta_{j}-\mu_{s, n}\left(\zeta_{j}\right)\right) d m_{s}
\end{aligned}
$$

Combining this and (7.23), we get

$$
\begin{aligned}
& \left|\sum_{i=0}^{j-1} K_{i, j}-\sum_{i=0}^{j-1} \int\left(\psi_{i}-\mu_{s, n}\left(\psi_{i}\right)\right)\left(\zeta_{j}-\mu_{s, n}\left(\zeta_{j}\right)\right) d m_{s}\right|= \\
& =\left(\hat{\mathcal{L}}_{s}^{n} \mathbb{1}(x)\right)^{-1} \mid \hat{\mathcal{L}}_{s}^{n-j}\left(\sum_{i=0}^{j-1} \hat{\mathcal{L}}_{s}^{j-i}\left(\left(\psi-\mu_{s, n}\left(\psi_{i}\right)\right) \hat{\mathcal{L}}_{s}^{i}(\mathbb{1})\right)\left(\zeta-\mu_{s, n}\left(\zeta_{j}\right)\right)\right)(x)- \\
& \quad-\rho_{s}(x) \int \sum_{i=0}^{j-1} \hat{\mathcal{L}}_{s}^{j-i}\left(\left(\psi-\mu_{s, n}\left(\psi_{i}\right)\right) \hat{\mathcal{L}}_{s}^{i}(\mathbb{1})\right)\left(\zeta-\mu_{s, n}\left(\zeta_{j}\right)\right) d m_{s}+
\end{aligned}
$$

$$
\begin{aligned}
& +\left(\rho_{s}(x)-\hat{\mathcal{L}}_{s}^{n} \mathbb{1}(x)\right) \int \sum_{i=0}^{j-1} \hat{\mathcal{L}}_{s}^{j-i}\left(\left(\psi-\mu_{s, n}\left(\psi_{i}\right)\right) \hat{\mathcal{L}}_{s}^{i}(\mathbb{1})\right)\left(\zeta-\mu_{s, n}\left(\zeta_{j}\right)\right) d m_{s} \mid \\
& \leq\left(\hat{\mathcal{L}}_{s}^{n} \mathbb{1}(x)\right)^{-1}\left(C_{2} \theta^{n-j-1}+\right. \\
& \left.\left.\quad+C \theta^{n} \mid \int \hat{\mathcal{L}}_{s}\left(\sum_{i=0}^{j-1} \hat{\mathcal{L}}_{s}^{j-i}\left(\psi-\mu_{s, n}\left(\psi_{i}\right)\right) \hat{\mathcal{L}}_{s}^{i}(\mathbb{1})\right)\left(\zeta-\mu_{s, n}\left(\zeta_{j}\right)\right)\right) d m_{s} \mid\right) \\
& \left.\leq 4 g^{-1}\left(C_{2} \theta^{n-j-1}+C \theta^{n} \| \hat{\mathcal{L}}_{s}\left(\sum_{i=0}^{j-1} \hat{\mathcal{L}}_{s}^{j-i}\left(\psi-\mu_{s, n}\left(\psi_{i}\right)\right) \hat{\mathcal{L}}_{s}^{i}(\mathbb{1})\right)\left(\zeta-\mu_{s, n}\left(\zeta_{j}\right)\right)\right) \|_{\beta}\right) \\
& \leq 4 g^{-1}\left(C_{2} \theta^{n-j-1}+C \theta^{n} C_{2}\right) \leq 8 C_{2} C g^{-1} \theta^{n-j-1} .
\end{aligned}
$$

Let us now deal with the case when $i=j$. It follows from Remark 7.10, Lemma 7.7. Lemma 7.9 and (7.19) that

$$
\begin{aligned}
& \left\|\hat{\mathcal{L}}_{s}\left(\left(\psi-\mu_{s, n}\left(\psi_{j}\right)\right) \hat{\mathcal{L}}_{s}^{j}(\mathbb{1})\left(\zeta-\mu_{s, n}\left(\zeta_{j}\right)\right)\right)\right\|_{\beta}= \\
& =\| \hat{\mathcal{L}}_{s}\left(\psi \zeta \hat{\mathcal{L}}_{s}^{j}(\mathbb{1})\right)-\mu_{s, n}\left(\zeta_{j}\right) \hat{\mathcal{L}}_{s}\left(\psi \mathcal{L}_{s}^{j}(\mathbb{1})\right)-\mu_{s, n}\left(\psi_{j}\right) \hat{\mathcal{L}}_{s}\left(\zeta \mathcal{L}_{s}^{j}(\mathbb{1})\right)+ \\
& \quad+\mu_{s, n}\left(\psi_{j}\right) \mu_{s, n}\left(\zeta_{j}\right) \hat{\mathcal{L}}_{s}^{j+1}(\mathbb{1}) \|_{\beta} \\
& \quad \leq\left\|\hat{\mathcal{L}}_{s}\left(\psi \zeta \hat{\mathcal{L}}_{s}^{j}(\mathbb{1})\right)\right\|_{\beta}+\left|\mu_{s, n}\left(\zeta_{j}\right)\left\|\hat{\mathcal{L}}_{s}\left(\psi \mathcal{L}_{s}^{j}(\mathbb{1})\right)\right\|_{\beta}+\right| \mu_{s, n}\left(\psi_{j}\right) \mid\left\|\hat{\mathcal{L}}_{s}\left(\zeta \mathcal{L}_{s}^{j}(\mathbb{1})\right)\right\|_{\beta}+ \\
& \quad+\mid \mu_{s, n}\left(\psi_{j}\right)\left\|\mu_{s, n}\left(\zeta_{j}\right)\right\| \hat{\mathcal{L}}_{s}^{j+1}(\mathbb{1}) \|_{\beta} \\
& \leq C \Gamma(\psi \zeta)+4 g^{-1} C^{2} \Gamma(\zeta) \Gamma(\psi) C+4 g^{-1} C^{2} \Gamma(\psi) \Gamma(\zeta) C+16 g^{-2} C^{4} \Gamma(\zeta) \Gamma(\psi) C \\
& =C\left(\Gamma(\psi \zeta)+C^{2} g^{-1} \Gamma(\zeta) \Gamma(\psi)\left(2+4 C^{2} g^{-1}\right)\right)
\end{aligned}
$$


Denote this last constant by $C_{3}$. We then have

$$
\begin{aligned}
\mid \int \hat{\mathcal{L}}_{s}\left(\left(\psi-\mu_{s, n}\left(\psi_{j}\right)\right) \hat{\mathcal{L}}_{s}^{j}(\mathbb{1})\right. & \left.\left(\zeta-\mu_{s, n}\left(\zeta_{j}\right)\right)\right) d m_{s} \mid \\
& \leq\left\|\hat{\mathcal{L}}_{s}\left(\left(\psi-\mu_{s, n}\left(\psi_{j}\right)\right) \hat{\mathcal{L}}_{s}^{j}(\mathbb{1})\left(\zeta-\mu_{s, n}\left(\zeta_{j}\right)\right)\right)\right\|_{\infty} \\
& \leq\left\|\hat{\mathcal{L}}_{s}\left(\left(\psi-\mu_{s, n}\left(\psi_{j}\right)\right) \hat{\mathcal{L}}_{s}^{j}(\mathbb{1})\left(\zeta-\mu_{s, n}\left(\zeta_{j}\right)\right)\right)\right\|_{\beta} \leq C_{3} .
\end{aligned}
$$

Applying now Lemma 7.7, we therefore get

$$
\begin{aligned}
& \| \hat{\mathcal{L}}_{s}^{n-j}\left(\left(\psi-\mu_{s, n}\left(\psi_{j}\right)\right) \hat{\mathcal{L}}_{s}^{j}(\mathbb{1})\left(\zeta-\mu_{s, n}\left(\zeta_{j}\right)\right)\right)- \\
& \quad-\hat{\mathcal{L}}_{s}^{n}(\mathbb{1}) \int\left(\psi-\mu_{s, n}\left(\psi_{j}\right)\right) \hat{\mathcal{L}}_{s}^{j}(\mathbb{1})\left(\zeta-\mu_{s, n}\left(\zeta_{j}\right)\right) d m_{s} \|_{\beta} \\
& \leq \| \hat{\mathcal{L}}_{s}^{n-j-1} \hat{\mathcal{L}}_{s}\left(\left(\psi-\mu_{s, n}\left(\psi_{j}\right)\right) \hat{\mathcal{L}}_{s}^{j}(\mathbb{1})\left(\zeta-\mu_{s, n}\left(\zeta_{j}\right)\right)\right)- \\
& \quad-\rho_{s} \int \hat{\mathcal{L}}_{s}\left(\left(\psi-\mu_{s, n}\left(\psi_{j}\right)\right) \hat{\mathcal{L}}_{s}^{j}(\mathbb{1})\left(\zeta-\mu_{s, n}\left(\zeta_{j}\right)\right)\right) d m_{s}+ \\
& +\left(\rho_{s}-\hat{\mathcal{L}}_{s}^{n}(\mathbb{1})\right) \int \hat{\mathcal{L}}_{s}\left(\left(\psi-\mu_{s, n}\left(\psi_{j}\right)\right) \hat{\mathcal{L}}_{s}^{j}(\mathbb{1})\left(\zeta-\mu_{s, n}\left(\zeta_{j}\right)\right)\right) d m_{s} \|_{\beta} \\
& =\| S_{s}^{n-j-1} \hat{\mathcal{L}}_{s}\left(\left(\psi-\mu_{s, n}\left(\psi_{j}\right)\right) \hat{\mathcal{L}}_{s}^{j}(\mathbb{1})\left(\zeta-\mu_{s, n}\left(\zeta_{j}\right)\right)\right)+ \\
& +S_{s}^{n}(\mathbb{1}) \int \hat{\mathcal{L}}_{s}\left(\left(\psi-\mu_{s, n}\left(\psi_{j}\right)\right) \hat{\mathcal{L}}_{s}^{j}(\mathbb{1})\left(\zeta-\mu_{s, n}\left(\zeta_{j}\right)\right)\right) d m_{s} \|_{\beta} \\
& \leq C \theta^{n-j-1}\left\|\hat{\mathcal{L}}_{s}\left(\left(\psi-\mu_{s, n}\left(\psi_{j}\right)\right) \hat{\mathcal{L}}_{s}^{j}(\mathbb{1})\left(\zeta-\mu_{s, n}\left(\zeta_{j}\right)\right)\right)\right\|_{\beta}+ \\
& +C \theta^{n}\left|\int \hat{\mathcal{L}}_{s}\left(\left(\psi-\mu_{s, n}\left(\psi_{j}\right)\right) \hat{\mathcal{L}}_{s}^{j}(\mathbb{1})\left(\zeta-\mu_{s, n}\left(\zeta_{j}\right)\right)\right) d m_{s}\right| \\
& \leq C \theta^{n-j-1} C_{3}+C \theta^{n} C_{3} \leq 2 C C_{3} \theta^{n-j-1} .
\end{aligned}
$$

But

$$
\begin{aligned}
\int\left(\psi-\mu_{s, n}\left(\psi_{j}\right)\right) \hat{\mathcal{L}}_{s}^{j}(\mathbb{1}) & \left(\zeta-\mu_{s, n}\left(\zeta_{j}\right)\right) d m_{s}= \\
& =\int \hat{\mathcal{L}}_{s}^{j}\left(\left(\psi_{j}-\mu_{s, n}\left(\psi_{j}\right)\right)\left(\zeta_{j}-\mu_{s, n}\left(\zeta_{j}\right)\right)\right) d m_{s} \\
& =\int\left(\psi_{j}-\mu_{s, n}\left(\psi_{j}\right)\right)\left(\zeta_{j}-\mu_{s, n}\left(\zeta_{j}\right)\right) d m_{s}
\end{aligned}
$$

and consequently

$$
\begin{aligned}
\left|K_{j, j}-\int\left(\psi_{j}-\mu_{s, n}\left(\psi_{j}\right)\right)\left(\zeta_{j}-\mu_{s, n}\left(\zeta_{j}\right)\right) d m_{s}\right| & \leq 2 C C_{3}\left(\hat{\mathcal{L}}_{s}^{n}(\mathbb{1})(x)\right)^{-1} \theta^{n-j-1} \\
& \leq 8 C C_{3} g^{-1} \theta^{n-j-1}
\end{aligned}
$$

Combining this and (7.24), we get

$$
\left|\sum_{i=0}^{j} K_{i, j}-\sum_{i=0}^{j} \int\left(\psi_{i}-\mu_{s, n}\left(\psi_{i}\right)\right)\left(\zeta_{i}-\mu_{s, n}\left(\zeta_{i}\right)\right) d m_{s}\right| \leq 8 C g^{-1}\left(C_{2}+C_{3}\right) \theta^{n-j-1}
$$


Hence, this step is concluded by the following.

$$
\begin{aligned}
\mid \Delta_{n}(s)- & \sum_{i=0}^{n-1} \sum_{j=0}^{n-1} \int\left(\psi_{i}-\mu_{s, n}\left(\psi_{i}\right)\right)\left(\zeta_{j}-\mu_{s, n}\left(\zeta_{j}\right)\right) d m_{s} \mid= \\
= & \mid \sum_{j=0}^{n-1}\left(\sum_{i=0}^{j} K_{i, j}-\sum_{i=0}^{j} \int\left(\psi_{i}-\mu_{s, n}\left(\psi_{i}\right)\right)\left(\zeta_{j}-\mu_{s, n}\left(\zeta_{j}\right)\right) d m_{s}\right)+ \\
& +\sum_{k=0}^{n-1}\left(\sum_{l=0}^{k-1} K_{k, l}-\sum_{i=0}^{j} \int\left(\psi_{k}-\mu_{s, n}\left(\psi_{k}\right)\right)\left(\zeta_{l}-\mu_{s, n}\left(\zeta_{l}\right)\right) d m_{s}\right) \mid \\
\leq & \sum_{j=0}^{n-1} 16 C g^{-1}\left(C_{2}+C_{3}\right) \theta^{n-j-1} \leq \sum_{u=0}^{\infty} 16 C g^{-1}\left(C_{2}+C_{3}\right) \theta^{u} \\
= & 16 C g^{-1}\left(C_{2}+C_{3}\right)(1-\theta)^{-1}<\infty .
\end{aligned}
$$

Step 3: The third step is to show that the measures $\mu_{s, n}$ can be all replaced by $m_{s}$ and the absolute value of the difference remains bounded. This is accomplished in (7.29). Utilizing now the formula $a b-c d=(a-c) b+c(b-d)$, we get

$$
\begin{aligned}
& \left|\int\left(\psi_{i}-\mu_{s, n}\left(\psi_{i}\right)\right)\left(\zeta_{j}-\mu_{s, n}\left(\zeta_{j}\right)\right) d m_{s}-\int\left(\psi_{i}-m_{s}\left(\psi_{i}\right)\right)\left(\zeta_{j}-m_{s}\left(\zeta_{j}\right)\right) d m_{s}\right| \\
& =\left|\int\left(\left(\psi_{i}-\mu_{s, n}\left(\psi_{i}\right)\right)\left(\zeta_{j}-\mu_{s, n}\left(\zeta_{j}\right)\right)-\left(\psi_{i}-m_{s}\left(\psi_{i}\right)\right)\left(\zeta_{j}-m_{s}\left(\zeta_{j}\right)\right)\right) d m_{s}\right| \\
& =\mid \int\left(\left(m_{s}\left(\psi_{i}\right)-\mu_{s, n}\left(\psi_{i}\right)\right)\left(\zeta_{j}-\mu_{s, n}\left(\zeta_{j}\right)\right)+\right. \\
& \quad+\left(\psi_{i}-m_{s}\left(\psi_{i}\right)\right)\left(m_{s}\left(\zeta_{j}\right)-\mu_{s, n}\left(\zeta_{j}\right)\right) d m_{s} \mid \\
& =\mid\left(m_{s}\left(\psi_{i}\right)-\mu_{s, n}\left(\psi_{i}\right)\right)\left(m_{s}\left(\zeta_{j}\right)-\mu_{s, n}\left(\zeta_{j}\right)\right)+ \\
& \quad+\left(m_{s}\left(\psi_{i}\right)-m_{s}\left(\psi_{i}\right)\right)\left(m_{s}\left(\zeta_{j}\right)-\mu_{s, n}\left(\zeta_{j}\right) \mid\right. \\
& =\left|\left(m_{s}\left(\psi_{i}\right)-\mu_{s, n}\left(\psi_{i}\right)\right)\left(m_{s}\left(\zeta_{j}\right)-\mu_{s, n}\left(\zeta_{j}\right)\right)\right| \\
& =\left|m_{s}\left(\psi_{i}\right)-\mu_{s, n}\left(\psi_{i}\right)\right| \cdot\left|m_{s}\left(\zeta_{j}\right)-\mu_{s, n}\left(\zeta_{j}\right)\right| .
\end{aligned}
$$

Since

$$
\begin{aligned}
m_{s}\left(\psi_{i}\right)-\mu_{s, n}\left(\psi_{i}\right) & =\int\left(\psi_{i}-\mu_{s, n}\left(\psi_{i}\right)\right) d m_{s}=\int \hat{\mathcal{L}}_{s}^{i}\left(\psi_{i}-\mu_{s, n}\left(\psi_{i}\right)\right) d m_{s} \\
& =\int\left(\psi-\mu_{s, n}\left(\psi_{i}\right)\right) \hat{\mathcal{L}}_{s}^{i}(\mathbb{1}) d m_{s}
\end{aligned}
$$

it follows from (7.21) that

$$
\left|m_{s}\left(\psi_{i}\right)-\mu_{s, n}\left(\psi_{i}\right)\right| \leq 8 C^{2}(g \theta)^{-1} \Gamma(\psi) \theta^{n-i} .
$$

Similarly

$$
\left|m_{s}\left(\zeta_{j}\right)-\mu_{s, n}\left(\zeta_{j}\right)\right| \leq 8 C^{2}(g \theta)^{-1} \mid \Gamma(\zeta) \theta^{n-j}
$$


Combining these last two estimates along with (7.27) and (7.28), we get

$$
\begin{aligned}
& \left.\mid \Delta_{n}(s)-\sum_{i=0}^{n-1} \sum_{j=0}^{n-1} \int\left(\psi_{i}-m_{s}\left(\psi_{i}\right)\right)\left(\zeta_{j}-m_{s}\left(\zeta_{j}\right)\right)\right) d m_{s} \mid \leq \\
& \leq 16 C g^{-1}\left(C_{2}+C_{3}\right)(1-\theta)^{-1}+\left(8 C^{2}(g \theta)^{-1}\right)^{2} \Gamma(\psi) \Gamma(\zeta) \sum_{i, j=0}^{n-1} \theta^{n-i} \theta^{n-j} \\
& \leq 16 C g^{-1}\left(C_{2}+C_{3}\right)(1-\theta)^{-1}+\left(8 C^{2}(g \theta)^{-1}\right)^{2}(1-\theta)^{-2} \Gamma(\psi) \Gamma(\zeta):=E_{1} .
\end{aligned}
$$

Step 4: In this step we show the integrals $m_{s}\left(\psi_{i}\right)$ and $m_{s}\left(\zeta_{j}\right)$ can be replaced respectively by $\mu_{s}\left(\psi_{i}\right)$ and $\mu_{s}\left(\zeta_{j}\right)$. This is done in (7.32) and (17.33). Since for every $k \geq 0$ and every loosely tame function $\omega: \mathcal{J}(f) \rightarrow \mathbb{R}$, we have

$$
\begin{aligned}
m_{s}\left(\omega_{k}\right) & =m_{s}\left(\hat{\mathcal{L}}_{s}^{k}\left(\omega_{k}\right)\right)=m_{s}\left(\omega \hat{\mathcal{L}}_{s}^{k}(\mathbb{1})\right)=m_{s}\left(\omega g+\omega S_{s}^{k}(\mathbb{1})\right) \\
& =\mu_{s}(\omega)+m_{s}\left(\omega S_{s}^{k}(\mathbb{1})\right)=\mu_{s}\left(\omega_{k}\right)+m_{s}\left(\omega S_{s}^{k}(\mathbb{1})\right)
\end{aligned}
$$

it follows from Lemma 7.11 and Lemma 7.7 that

$$
\left|m_{s}\left(\omega_{k}\right)-\mu_{s}\left(\omega_{k}\right)\right| \leq\left\|S_{s}^{k}(\mathbb{1})\right\|_{\infty} m_{s}(|\omega|) \leq\left\|S_{s}^{k}(\mathbb{1})\right\|{ }_{\beta} \Gamma(|\omega|) \leq C \Gamma(|\omega|) \theta^{k}
$$

for all $s \in(-\eta, \eta)$. We also have

$$
\begin{aligned}
\int\left(\omega_{k}-\mu_{s}\left(\omega_{k}\right)\right) d m_{s} & =\int \hat{\mathcal{L}}_{s}^{k}(\mathbb{1})\left(\omega-\mu_{s}(\omega)\right) d m_{s}=\int\left(\rho_{s}+S_{s}^{k}(\mathbb{1})\right)\left(\omega-\mu_{s}(\omega)\right) d m_{s} \\
& =\int \omega \rho_{s} d m_{s}-\mu_{s}(\omega) \int \rho_{s} d m_{s}+\int S_{s}^{k}(\mathbb{1})\left(\omega-\mu_{s}(\omega)\right) d m_{s} \\
& =\mu_{s}(\omega)-\mu_{s}(\omega)+\int S_{s}^{k}(\mathbb{1})\left(\omega-\mu_{s}(\omega)\right) d m_{s} \\
& =\int S_{s}^{k}(\mathbb{1})\left(\omega-\mu_{s}(\omega)\right) d m_{s}
\end{aligned}
$$

Hence, using Lemma 7.7 and Lemma 7.11, we obtain

$$
\begin{aligned}
\mid \int\left(\omega_{k}-\right. & \left.\mu_{s}\left(\omega_{k}\right)\right) d m_{s}\left|\leq \int\right| S_{s}^{k}(\mathbb{1})|| \omega-\mu_{s}(\omega) \mid d m_{s} \\
& \leq \int|| S_{s}^{k}(\mathbb{1}) \|_{\beta}\left(|\omega|+\left|\mu_{s}(\omega)\right|\right) d m_{s} \leq C \theta^{k} \int\left(|\omega|+C m_{s}(|\omega|)\right) d m_{s} \\
& =C(1+C) m_{s}(|\omega|) \theta^{k} \leq C(1+C) \Gamma(|\omega|) \theta^{k} .
\end{aligned}
$$


Therefore, utilizing (7.30), (7.31), Lemma 7.7 and Lemma 7.11 for every $0 \leq i \leq j$, we get that

$$
\begin{aligned}
\mid \int\left(\left(\psi_{i}-\right.\right. & \left.\left.\mu_{s}\left(\psi_{i}\right)\right)\left(\zeta_{j}-\mu_{s}\left(\zeta_{j}\right)\right)-\left(\psi_{i}-m_{s}\left(\psi_{i}\right)\right)\left(\zeta_{j}-m_{s}\left(\zeta_{j}\right)\right)\right) d m_{s} \mid \leq \\
= & \mid \int\left(\left(m_{s}\left(\psi_{i}\right)-\mu_{s}\left(\psi_{i}\right)\right)\left(\zeta_{j}-\mu_{s}\left(\zeta_{j}\right)\right)+\right. \\
& \left.\quad+\left(\psi_{i}-m_{s}\left(\psi_{i}\right)\right)\left(m_{s}\left(\zeta_{j}\right)-\mu_{s}\left(\zeta_{j}\right)\right)\right) d m_{s} \mid \\
\leq & \left|\int\left(\zeta_{j}-\mu_{s}\left(\zeta_{j}\right)\right)\left(m_{s}\left(\psi_{i}\right)-\mu_{s}\left(\psi_{i}\right)\right) d m_{s}\right|+ \\
& \quad+\int\left|\psi_{i}-m_{s}\left(\psi_{i}\right)\right|\left|m_{s}\left(\zeta_{j}\right)-\mu_{s}\left(\zeta_{j}\right)\right| d m_{s} \mid \\
\leq & \left|\int\left(\zeta_{j}-\mu_{s}\left(\zeta_{j}\right)\right) m_{s}\left(\psi S_{s}^{i}(\mathbb{1})\right) d m_{s}\right|+\int\left|\psi_{i}-m_{s}\left(\psi_{i}\right)\right| d m_{s} C \Gamma(|\zeta|) \theta^{j} \\
\leq & \left|m_{s}\left(\psi S_{s}^{i}(\mathbb{1})\right) \int\left(\zeta_{j}-\mu_{s}\left(\zeta_{j}\right)\right) d m_{s}\right|+2 m_{s}\left(\left|\psi_{i}\right|\right) C \Gamma(|\zeta|) \theta^{j} \\
= & \left|m_{s}\left(\psi S_{s}^{i}(\mathbb{1})\right)\right| \cdot\left|m_{s}\left(\zeta_{j}-\mu_{s}\left(\zeta_{j}\right)\right)\right|+2 m_{s}\left(|\psi| \hat{\mathcal{L}}_{s}^{i}(\mathbb{1})\right) C \Gamma(|\zeta|) \theta^{j} \\
\leq & C \theta^{i} m_{s}(|\psi|) C(1+C) \Gamma(|\zeta|) \theta^{j}+2 C^{2} \Gamma(|\psi|) \Gamma(|\zeta|) \theta^{j} \\
\leq & C^{2}(1+C) \Gamma(|\psi|) \Gamma(|\zeta|) \theta^{i} \theta^{j}+2 C^{2} \Gamma(|\psi|) \Gamma(|\zeta|) \theta^{j} \\
\leq & C^{2}(3+C) \Gamma(|\psi|) \Gamma(|\zeta|) \theta^{j} .
\end{aligned}
$$

Hence, putting

$$
E_{2}=2 C^{2}(3+C) \Gamma(|\psi|) \Gamma(|\zeta|) \sum_{j=0}^{\infty} j \theta^{j}<\infty
$$

we get

$$
\begin{aligned}
\mid \sum_{i, j=0}^{n-1} \int\left(\psi_{i}-\mu_{s}\left(\psi_{i}\right)\right) & \left(\zeta_{j}-\mu_{s}\left(\zeta_{j}\right)\right) d m_{s}- \\
& -\sum_{i, j=0}^{n-1} \int\left(\psi_{i}-m_{s}\left(\psi_{i}\right)\right)\left(\zeta_{j}-m_{s}\left(\zeta_{j}\right)\right) d m_{s} \mid \leq E_{2}
\end{aligned}
$$

for all $s \in(-\eta, \eta)$ and all $n \geq 1$. Thus, invoking (7.29), we get

$$
\left.\mid \Delta_{n}(s)-\sum_{i=0}^{n-1} \sum_{j=0}^{n-1} \int\left(\psi_{i}-\mu_{s}\left(\psi_{i}\right)\right)\left(\zeta_{j}-\mu_{s}\left(\zeta_{j}\right)\right)\right) d m_{s} \mid \leq E_{1}+E_{2} .
$$

Step 5: This step is to show that the measure $m_{s}$ above can be replaced by $\mu_{s}$. This is done in (7.36) and (7.37). Put $\bar{\psi}=\psi-\mu_{s}(\psi)$ and $\bar{\zeta}=\zeta-\mu_{s}(\zeta)$. For all 
$0 \leq i<j$ we then have

$$
\begin{aligned}
\int \bar{\psi}_{i} \bar{\zeta}_{j} d m_{s} & =\int\left(\bar{\psi} \bar{\zeta}_{j-i}\right) \circ f^{i} d m_{s}=\int \bar{\psi} \bar{\zeta}_{j-i} \hat{\mathcal{L}}_{s}^{i}(\mathbb{1}) d m_{s} \\
& =\int \bar{\psi} \bar{\zeta}_{j-i}\left(\rho_{s}+S_{s}^{i}(\mathbb{1})\right) d m_{s}=\int \bar{\psi} \bar{\zeta}_{j-i} \rho_{s} d m_{s}+\int \bar{\psi} \bar{\zeta}_{j-i} S_{s}^{i}(\mathbb{1}) d m_{s} \\
& =\int \bar{\psi} \bar{\zeta}_{j-i} d \mu_{s}+\int \hat{\mathcal{L}}_{s}^{j-i}\left(\bar{\psi} \bar{\zeta}_{j-i} S_{s}^{i}(\mathbb{1})\right) d m_{s} \\
& =\int \bar{\psi}_{i} \bar{\zeta}_{j} d \mu_{s}+\int \bar{\zeta} \hat{\mathcal{L}}_{s}^{j-i}\left(\bar{\psi} S_{s}^{i}(\mathbb{1})\right) d m_{s} \\
& =\int \bar{\psi}_{i} \bar{\zeta}_{j} d \mu_{s}+\int \bar{\zeta} \hat{\mathcal{L}}_{s}^{j-i-1}\left(\hat{\mathcal{L}}_{s}\left(\bar{\psi} S_{s}^{i}(\mathbb{1})\right)\right) d m_{s} \\
& =\int \bar{\psi}_{i} \bar{\zeta}_{j} d \mu_{s}+\int \bar{\zeta} m_{s}\left(\hat{\mathcal{L}}_{s}\left(\bar{\psi} S_{s}^{i}(\mathbb{1})\right)\right) \rho_{s} d m_{s} \\
& +\int \bar{\zeta} S_{s}^{j-i-1}\left(\hat{\mathcal{L}}_{s}\left(\bar{\psi} S_{s}^{i}(\mathbb{1})\right)\right) d m_{s} \\
& \left.=\int \bar{\psi}_{i} \bar{\zeta}_{j} d \mu_{s}+m_{s}\left(\bar{\psi} S_{s}^{i}(\mathbb{1})\right)\right) \int \bar{\zeta} d \mu_{s}+\int \bar{\zeta} S_{s}^{j-i-1}\left(\hat{\mathcal{L}}_{s}\left(\bar{\psi} S_{s}^{i}(\mathbb{1})\right)\right) d m_{s} \\
& =\int \bar{\psi}_{i} \bar{\zeta}_{j} d \mu_{s}+\int \bar{\zeta} S_{s}^{j-i-1}\left(\hat{\mathcal{L}}_{s}\left(\bar{\psi} S_{s}^{i}(\mathbb{1})\right)\right) d m_{s} .
\end{aligned}
$$

Let us now estimate the absolute value of the second summand. Using Lemma 7.7 Lemma 7.9 and Lemma 7.11, we obtain

$$
\begin{aligned}
\left|\int \bar{\zeta} S_{s}^{j-i-1}\left(\hat{\mathcal{L}}_{s}\left(\bar{\psi} S_{s}^{i}(\mathbb{1})\right)\right) d m_{s}\right| & \leq \int|\bar{\zeta}|\left|S_{s}^{j-i-1}\left(\hat{\mathcal{L}}_{s}\left(\bar{\psi} S_{s}^{i}(\mathbb{1})\right)\right)\right| d m_{s} \\
& \leq \int|\bar{\zeta}||| S_{s}^{j-i-1}\left(\hat{\mathcal{L}}_{s}\left(\bar{\psi} S_{s}^{i}(\mathbb{1})\right)\right) \|_{\infty} d m_{s} \\
& \leq\left\|S_{s}^{j-i-1}\left(\hat{\mathcal{L}}_{s}\left(\bar{\psi} S_{s}^{i}(\mathbb{1})\right)\right)\right\|_{\beta} \int|\bar{\zeta}| d m_{s} \\
& \leq C \theta^{j-i-1}\left\|\hat{\mathcal{L}}_{s}\left(\bar{\psi} S_{s}^{i}(\mathbb{1})\right)\right\|_{\beta} \int\left(|\zeta|+\left|\mu_{s}(\zeta)\right|\right) d m_{s} \\
& \leq C \theta^{j-i-1} \Gamma(\bar{\psi})\left\|S_{s}^{i}(\mathbb{1})\right\|_{\beta}(\Gamma(|\zeta|)+C \Gamma(|\zeta|)) \\
& \leq C^{2}(1+C) \Gamma(\bar{\psi}) \Gamma(|\zeta|) \theta^{-1} \theta^{j}
\end{aligned}
$$

Hence, if $0 \leq i<j$, then

$$
\left|\int \bar{\psi}_{i} \bar{\zeta}_{j} d m_{s}-\int \bar{\psi}_{i} \bar{\zeta}_{j} d \mu_{s}\right| \leq C^{2}(1+C) \theta^{-1} \Gamma(\bar{\psi}) \Gamma(|\zeta|) \theta^{j} .
$$

Now, for every $j \geq 0$ we have

$$
\begin{aligned}
\int \bar{\psi}_{j} \bar{\zeta}_{j} d m_{s} & =\int \hat{\mathcal{L}}_{s}^{j}(\mathbb{1}) \bar{\psi} \bar{\zeta} d m_{s}=\int \bar{\psi} \bar{\zeta}\left(\rho_{s}+S_{s}^{j}(\mathbb{1})\right) d m_{s} \\
& =\int \bar{\psi} \bar{\zeta} \rho_{s} d m_{s}+\int \bar{\psi} \bar{\zeta} S_{s}^{j}(\mathbb{1}) d m_{s} \\
& =\int \bar{\psi}_{j} \bar{\zeta}_{j} d \mu_{s}+\int \hat{\mathcal{L}}_{s}\left(\bar{\psi} \bar{\zeta} S_{s}^{j}(\mathbb{1})\right) d m_{s}
\end{aligned}
$$


Hence, utilising Lemma 7.9. Remark 7.10 and Lemma 7.7, we obtain

$$
\begin{aligned}
\mid \int \bar{\psi}_{j} \bar{\zeta}_{j} d m_{s} & -\int \bar{\psi}_{j} \bar{\zeta}_{j} d \mu_{s}|=| \int \hat{\mathcal{L}}_{s}\left(\bar{\psi} \bar{\zeta} S_{s}^{j}(\mathbb{1})\right) d m_{s} \mid \\
& \leq\left\|\hat{\mathcal{L}}_{s}\left(\bar{\psi} \bar{\zeta} S_{s}^{j}(\mathbb{1})\right)\right\|_{\beta} \leq \Gamma(\bar{\psi} \bar{\zeta})|| S_{s}^{j}(\mathbb{1}) \|_{\beta} \leq C \Gamma(\bar{\psi} \bar{\zeta}) \theta^{j} .
\end{aligned}
$$

Now, by Lemma 7.9, Lemma 7.11 and Lemma 7.7, we get

$$
\begin{aligned}
\Gamma(\bar{\psi} \bar{\zeta})= & \Gamma\left(\psi \zeta-\mu_{s}(\psi) \zeta-\mu_{s}(\zeta) \psi+\mu_{s}(\psi) \mu_{s}(\zeta)\right) \\
\leq & \Gamma(\psi \zeta)+\left|\mu_{s}(\psi)\right| \Gamma(\zeta)+\left|\mu_{s}(\zeta)\right| \Gamma(\psi)+\left|\mu_{s}(\psi) \mu_{s}(\zeta)\right| \Gamma(\mathbb{1}) \\
\leq & \quad \Gamma(\psi \zeta)+\left\|\rho_{s}\right\|_{\infty} m_{s}(|\psi|) \Gamma(\zeta)+\left\|\rho_{s}\right\|_{\infty} m_{s}(|\zeta|) \Gamma(\psi)+ \\
& \quad+C\left\|\rho_{s}\right\|_{\infty}^{2} m_{s}(|\psi|) m_{s}(|\zeta|) \\
\leq & \Gamma(\psi \zeta)+\left\|\rho_{s}\right\|_{\beta} \Gamma(|\psi|) \Gamma(\zeta)+\left\|\rho_{s}\right\|_{\beta} \Gamma(|\zeta|) \Gamma(\psi)+C|| \rho_{s} \|_{\beta}^{2} \Gamma(|\psi|) \Gamma(|\zeta|) \\
\leq & \Gamma(\psi \zeta)+C \Gamma(|\psi|) \Gamma(\zeta)+C \Gamma(|\zeta|) \Gamma(\psi)+C^{3} \Gamma(|\psi|) \Gamma(|\zeta|)
\end{aligned}
$$

We can therefore conclude (7.35) by writing

$$
\begin{aligned}
\mid \int \bar{\psi}_{j} \bar{\zeta}_{j} d m_{s}- & \int \bar{\psi}_{j} \bar{\zeta}_{j} d \mu_{s} \mid \\
& \leq C\left(\Gamma(\psi \zeta)+C \Gamma(|\psi|) \Gamma(\zeta)+C \Gamma(|\zeta|) \Gamma(\psi)+C^{3} \Gamma(|\psi|) \Gamma(|\zeta|)\right) \theta^{j}
\end{aligned}
$$

Denoting by $E_{3}$ the maximum of coefficients of $\theta^{j}$ appearing in this inequality and in (7.34), we get that

$$
\left|\sum_{i, j=0}^{n-1}\left(\int \bar{\psi}_{i} \bar{\zeta}_{j} d m_{s}-\int \bar{\psi}_{i} \bar{\zeta}_{j} d \mu_{s}\right)\right| \leq E_{3} \sum_{k=0}^{\infty}(k+1) \theta^{k}:=E_{4}
$$

Combing this and (7.33), we obtain for all $n \geq 1$ and all $s \in(-\eta, \eta)$ that

$$
\left|\Delta_{n}(s)-\sum_{i=0}^{n-1} \sum_{j=0}^{n-1} \int\left(\psi_{i}-\mu_{s}\left(\psi_{i}\right)\right)\left(\zeta_{j}-\mu_{s}\left(\zeta_{j}\right)\right) d \mu_{s}\right| \leq E_{1}+E_{2}+E_{4} .
$$

Denote again $\psi-\mu(\psi)$ by $\bar{\psi}$ and $\zeta-\mu_{s}(\zeta)$ by $\bar{\zeta}$. If we knew that $\frac{1}{n} \sum_{i, j=0}^{n-1} \bar{\psi}_{i} \bar{\zeta}_{j} d \mu_{s}$ converged to $\sigma^{2}(\psi, \zeta)$ uniformly in $s \in(-\eta, \eta)$, (7.37) would finish the proof. We do it in the next step, Step 5, deriving simultaneously the second expression for $\sigma^{2}(\psi, \zeta)$. 
Step 6: We have

$$
\begin{aligned}
& \frac{1}{n} \int \sum_{i, j=0}^{n-1} \bar{\psi}_{i} \bar{\zeta}_{j} d \mu_{s}= \\
& =\frac{1}{n} \sum_{i=0}^{n-1} \sum_{j=i+1}^{n-1} \int \bar{\psi}_{i} \bar{\zeta}_{j} d \mu_{s}+\frac{1}{n} \sum_{j=0}^{n-1} \sum_{i=j+1}^{n-1} \int \bar{\psi}_{i} \bar{\zeta}_{j} d \mu_{s}+\frac{1}{n} \sum_{i=0}^{n-1} \int \bar{\psi}_{i} \bar{\zeta}_{i} d \mu_{s} \\
& =\frac{1}{n} \sum_{i=0}^{n-1} \int \bar{\psi} \bar{\zeta} d \mu_{s}+\frac{1}{n} \sum_{k=1}^{n-1}(n-1-k) \int \bar{\psi} \bar{\zeta}_{k} d \mu_{s}+\frac{1}{n} \sum_{k=1}^{n-1}(n-1-k) \int \overline{\zeta \psi}_{k} d \mu_{s} \\
& =\int \bar{\psi} \bar{\zeta} d \mu_{s}+\sum_{k=1}^{\infty} \int \bar{\psi} \bar{\zeta}_{k} d \mu_{s}-\frac{1}{n} \sum_{k=n}^{\infty} \int \bar{\psi} \bar{\zeta}_{k} d \mu_{s}-\frac{1}{n} \sum_{k=1}^{n-1}(k+1) \int \bar{\psi} \bar{\zeta}_{k} d \mu_{s}+ \\
& \quad+\sum_{k=1}^{\infty} \int \overline{\zeta \psi}_{k} d \mu_{s}-\frac{1}{n} \sum_{k=n}^{\infty} \int \overline{\zeta \psi} \bar{\psi}_{k} d \mu_{s}-\frac{1}{n} \sum_{k=1}^{n-1}(k+1) \int \overline{\zeta \psi}_{k} d \mu_{s} \\
& =\int \bar{\psi} \bar{\zeta}^{\infty} d \mu_{s}+\sum_{k=1}^{\infty} C_{s, k}(\psi, \zeta)+\sum_{k=1}^{\infty} C_{s, k}(\zeta, \psi)-\frac{1}{n} \sum_{k=n}^{\infty}\left(C_{s, k}(\psi, \zeta)+C_{s, k}(\zeta, \psi)\right)- \\
& \quad-\frac{1}{n} \sum_{k=1}^{n-1}(k+1)\left(C_{s, k}(\psi, \zeta)+C_{s, k}(\zeta, \psi)\right) .
\end{aligned}
$$

It now immediately follows from Lemma 7.12 that all the series appearing in the last part of this formula are uniformly convergent with respect to $s \in(-\eta, \eta)$, that the second two summands are uniformly bounded with respect to $s \in(-\eta, \eta)$, and the last two terms converge to 0 when $n \rightarrow \infty$ uniformly ith respect to $s \in(-\eta, \eta)$. Combining this with (7.37), we see that $\frac{1}{n} \Delta_{n}(s)$ converges to

$$
\int \bar{\psi} \bar{\zeta} d \mu_{s}+\sum_{k=1}^{\infty} C_{s, k}(\psi, \zeta)+\sum_{k=1}^{\infty} C_{s, k}(\zeta, \psi)
$$

uniformly ith respect to $s \in(-\eta, \eta)$. Applying now (7.17) completes the proof. 



\section{CHAPTER 8}

\section{Multifractal analysis}

Among other auxiliary results, we show here that the multifractal formalism holds for tame potentials $\phi=-t \log \left|f^{\prime}\right|_{\tau}+h$. The following notions are valid for any measures but we focus on the conformal measures $m_{\phi}$ and the equilibrium states $\mu_{\phi}$. The pointwise dimension of $\mu_{\phi}$ at $z \in \mathcal{J}(f)$ is given by

$$
d_{\mu_{\phi}}(z)=\lim _{r \rightarrow 0} \frac{\log \mu_{\phi}(D(z, r))}{\log r}
$$

provided this limit exists. Note that $d_{\mu_{\phi}}(z)=d_{m_{\phi}}(z)$ since $d \mu_{\phi}=\rho_{\phi} d m_{\phi}$ with $\rho_{\phi}$ a continuous non-vanishing function (Theorem 5.15 ). The object of the multifractal formalism is the geometric study of the level sets

$$
D_{\phi}(\alpha)=\left\{z \in \mathcal{J}_{r}(f) ; d_{\mu_{\phi}}(z)=\alpha\right\}
$$

and, in particular, we establish that the fractal spectrum

$$
\mathcal{F}_{\phi}(\alpha)=\operatorname{HD}\left(D_{\phi}(\alpha)\right)
$$

build a Legendre transform pair with the so called temperature function. As a main application we get that the fractal spectrum hehaves real analytic. The temperature function will be introduced and studied in Section 8.2 after having provided the Volume Lemma and Bowen's Formula.

\subsection{Hausdorff dimension of Gibbs states}

If $\mu$ is any probability measure of a metric space, then $\operatorname{HD}(\mu)$ denotes the Hausdorff dimension of this measure $\mu$ which is the infimum of the numbers $\operatorname{HD}(Y)$ taken over all Borel sets $Y$ such that $\mu(Y)=1$. If the local dimension $d_{\mu}(z)$ is constant a.e. equal to say $d_{\mu}$ then $\operatorname{HD}(\mu)=d_{\mu}$. If $\mu$ is a Borel probability $f$-invariant measure on $\mathcal{J}(f)$, then the number

$$
\chi_{\mu}=\int \log \left|f^{\prime}\right| d \mu
$$

is called the Lyapunov exponent of the map $f$ with respect to the measure $\mu$. The following result extends lots of similar results, usually referred as Volume Lemmas.

Theorem 8.1 (Volume Lemma). If $f: \mathbb{C} \rightarrow \hat{\mathbb{C}}$ is dynamically semi-regular and if $\phi$ is a tame potential, then for $\mu_{\phi}$-a.e. $z \in \mathcal{J}(f)$ the local dimension $d_{\mu_{\phi}}(z)$ exists and is equal to $h_{\mu_{\phi}} / \chi_{\mu_{\phi}}$. In particular

$$
\operatorname{HD}\left(\mu_{\phi}\right)=\frac{h_{\mu_{\phi}}}{\chi_{\mu_{\phi}}}
$$


Proof. In view of Birkhoff's ergodic theorem there exists a Borel set $X \subset$ $\mathcal{J}(f)$ such that $\mu_{\phi}(X)=1$ and

$$
\lim _{n \rightarrow \infty} \frac{1}{n} \log \left|\left(f^{n}\right)^{\prime}(x)\right|=\chi_{\mu_{\phi}} \text { and } \lim _{n \rightarrow \infty} \frac{1}{n} S_{n} \phi(x)=\int \phi d \mu_{\phi}
$$

for every $x \in X$. Fix $x \in X$ and $\varepsilon>0$. There then exists $k \geq 1$ such that

$$
\left|\frac{1}{n} \log \right|\left(f^{n}\right)^{\prime}(x)\left|-\chi_{\mu_{\phi}}\right|<\varepsilon
$$

for every $n \geq k$. Fix $r \in(0, \delta)$ and let $n=n(r) \geq 0$ be the largest integer such that

$$
D(x, r) \subset f_{x}^{-n}\left(D\left(f^{n}(x), \delta\right)\right) .
$$

Then $D(x, r)$ is not contained in $f_{x}^{-(n+1)}\left(D\left(f^{n+1}(x), \delta\right)\right)$ and it follows from the $\frac{1}{4}$-Koebe's distortion theorem that

$$
r \geq \frac{1}{4} \delta\left|\left(f^{n+1}\right)^{\prime}(x)\right|^{-1} .
$$

Taking $r>0$ sufficiently small, we may assume that $n \geq k$. Applying Lemma 5.20 and utilizing (8.6) along with Lemma 4.11, we get that

$$
\begin{aligned}
m_{\phi}(D(x, r)) & \leq \int_{D\left(f^{n}(x), \delta\right)} \exp \left(S_{n} \phi \circ f_{x}^{-n}-\mathrm{P}(\phi) n\right) d m_{\phi} \\
& \leq c \exp \left(S_{n} \phi(x)-\mathrm{P}(\phi) n\right) m_{\phi}\left(D\left(f^{n}(x), \delta\right)\right) \leq c \exp \left(S_{n} \phi(x)-\mathrm{P}(\phi) n\right) .
\end{aligned}
$$

Applying now (8.7) and (8.5), we obtain

$$
\begin{aligned}
\frac{\log m_{\phi}(D(x, r))}{\log r} & \geq \frac{\log c+S_{n} \phi(x)-\mathrm{P}(\phi) n}{\log r} \geq \frac{\log c+S_{n} \phi(x)-\mathrm{P}(\phi) n}{\log \delta-\log 4-\log \left|\left(f^{n+1}\right)^{\prime}(x)\right|} \\
& \geq \frac{\log c+S_{n} \phi(x)-\mathrm{P}(\phi) n}{\log \delta-\log 4-\left(\chi_{\mu_{\phi}}-\varepsilon\right)(n+1)} .
\end{aligned}
$$

Dividing now the numerator and the denominator of the last quotient by $n=n(r)$, letting $r \rightarrow 0$ (which implies that $n(r) \rightarrow \infty$ ) and using the second part of (8.4), we therefore get that

$$
\liminf _{r \rightarrow 0} \frac{\log \left(m_{\phi}(D(x, r))\right.}{\log r} \geq \frac{-\int \phi d \mu_{\phi}+\mathrm{P}(\phi)}{\chi_{\mu_{\phi}}} .
$$

Since, by Theorem [5.15 the measures $\mu_{\phi}$ and $m_{\phi}$ are equivalent with positive continuous Radon-Nikodym derivatives, we obtain for all $x \in X$ that

$$
\liminf _{r \rightarrow 0} \frac{\log \left(\mu_{\phi}(D(x, r))\right.}{\log r} \geq \frac{-\int \phi d \mu_{\phi}+\mathrm{P}(\phi)}{\chi_{\mu_{\phi}}} .
$$

For every $M>0$, let $J_{M}=\mathcal{J}(f) \cap D(0, M)$. Take $M$ so large that $\mu_{\phi}\left(J_{M}\right)>0$. Since the measure $m_{\phi}$ is positive on non-empty open subsets of $\mathcal{J}(f)$, we get that

$$
W:=\inf \left\{m_{\phi}\left(D(z, \delta): z \in J_{M}\right\}>0 .\right.
$$

In view of ergodicity of the measure $\mu_{\phi}$ and Birkhoff's ergodic theorem, there exists a Borel set $Y \subset X$ such that $\mu_{\phi}(Y)=1$ and

$$
\lim _{n \rightarrow \infty} \frac{1}{n} S_{n}\left(\mathbb{1}_{J_{M}}\right)(x)=\mu_{\phi}\left(J_{M}\right)>0
$$


for all $x \in Y$. In particular, if $\left\{n_{j}\right\}_{j=1}^{\infty}$ is the unbounded increasing sequence of all integers $n \geq 1$ such that $f^{n}(x) \in J_{M}$, then

$$
\lim _{j \rightarrow \infty} \frac{n_{j+1}}{n_{j}}=1
$$

Keep $x \in Y$ and let $l \geq 0$ be the least integer such that

$$
D(x, r) \supset f_{x}^{-i}\left(D\left(f^{i}(x), \delta\right)\right)
$$

for all $i \geq l$. Taking $r>0$ small enough, we may assume that $l>\max \left\{k, n_{1}\right\}$. There then exists a unique $j \geq 2$ such that

$$
n_{j-1}<l \leq n_{j} .
$$

Also $f_{x}^{-(l-1)}\left(D\left(f^{l-1}(x), \delta\right)\right)$ is not contained in $D(x, r)$, and it therefore follows from Koebe's distortion theorem that

$$
r \leq K \delta\left|\left(f^{l-1}\right)^{\prime}(x)\right|^{-1} .
$$

It follows from the definition of $l$ and formula (8.10) along with Lemma 4.11 that

$$
\begin{aligned}
m_{\phi}(D(x, r)) & \geq m_{\phi}\left(f_{x}^{-n_{j}}\left(D\left(f^{n_{j}}(x), \delta\right)\right)\right) \\
& =\int_{D\left(f^{n_{j}}(x), \delta\right)} \exp \left(S_{n_{j}} \phi \circ f_{x}^{-n_{j}}-\mathrm{P}(\phi) n_{j}\right) d m_{\phi} \\
& \geq c^{-1} \exp \left(S_{n_{j}} \phi(x)-\mathrm{P}(\phi) n_{j}\right) m_{\phi}\left(D\left(f^{n_{j}}(x), \delta\right)\right) \\
& \geq W c^{-1} \exp \left(S_{n_{j}} \phi(x)-\mathrm{P}(\phi) n_{j}\right) .
\end{aligned}
$$

Applying now (8.5), (8.7) and (8.10), we obtain

$$
\begin{aligned}
\frac{\log m_{\phi}(D(x, r))}{\log r} & \leq \frac{\log \left(\frac{W}{c}\right)+S_{n_{j}} \phi(x)-\mathrm{P}(\phi) n_{j}}{\log r} \leq \frac{\log \left(\frac{W}{c}\right)+S_{n_{j}} \phi(x)-\mathrm{P}(\phi) n_{j}}{\log (K \delta)-\log \left|\left(f^{l-1}\right)^{\prime}(x)\right|} \\
& \leq \frac{\log \left(\frac{W}{c}\right)+S_{n_{j}} \phi(x)-\mathrm{P}(\phi) n_{j}}{\log (K \delta)-\left(\chi_{\mu_{\phi}}+\varepsilon\right)(l-1)} \leq \frac{\log \left(\frac{W}{c}\right)+S_{n_{j}} \phi(x)-\mathrm{P}(\phi) n_{j}}{\log (K \delta)-\left(\chi_{\mu_{\phi}}+\varepsilon\right) n_{j-1}}
\end{aligned}
$$

Dividing now the numerator and the denominator of the last quotient by $n_{j-1}$ letting $r \rightarrow 0$ (which implies that $n_{j-1} \rightarrow \infty$ ) and using the second part of (8.4) along with (8.9), we therefore get that

$$
\limsup _{r \rightarrow 0} \frac{\log \left(m_{\phi}(D(x, r))\right.}{\log r} \leq \frac{-\int \phi d \mu_{\phi}+\mathrm{P}(\phi)}{\chi_{\mu_{\phi}}} .
$$

Since, by Theorem [5.15 the measures $\mu_{\phi}$ and $m_{\phi}$ are equivalent with positive continuous Radon-Nikodym derivatives, we obtain for all $x \in Y$ that

$$
\limsup _{r \rightarrow 0} \frac{\log \left(\mu_{\phi}(D(x, r))\right.}{\log r} \leq \frac{-\int \phi d \mu_{\phi}+\mathrm{P}(\phi)}{\chi_{\mu_{\phi}}} .
$$

Since, by Theorem 6.25, $\mathrm{P}(\phi)-\int \phi d \mu_{\phi}=\mathrm{h}_{\mu_{\phi}}$, combining this inequality with (8.8), completes the proof. 


\subsection{The temperature function}

Remember that, up to now, the metric and thus the number $\tau$ was any number such that $t>\frac{\rho}{\hat{\tau}}>\frac{\rho}{\alpha}$. The remaining part of this paper very much depends on the existence of the zero of the pressure function. We will see right now that the existence of that zero requires that $\tau$ is sufficiently close to $\underline{\alpha}_{2}$. In the following we can and do assume that this is always the case. Notice that the precise choice of the metric is without any importance since the pressure does not depend on it (Proposition 6.26).

Fix a tame potential $\phi=-t \log \left|f^{\prime}\right|_{\tau}+h$ and consider the two-parameter family of potentials

$$
\phi_{q, T}=-T \log \left|f^{\prime}\right|_{\tau}+q \phi ; \quad q, T \in \mathbb{R} .
$$

Note that $\phi_{q, T}$ is a $T+q t$-tame function.

Lemma 8.2. Let $f: \mathbb{C} \rightarrow \widehat{\mathbb{C}}$ be a dynamically regular function of order $\rho>0$ that has the divergence type property (Definition 2.4). There exists $\tau_{0}<\underline{\alpha}_{2}$ such that for every $\tau_{0}<\tau<\underline{\alpha}_{2}$ we have the following.

For every $q \in \mathbb{R}$ there exists a unique $T=T(q) \in \mathbb{R}$ such that $\mathrm{P}\left(\phi_{q, T}\right)=0$. In addition $T(q)>\frac{\rho}{\hat{\tau}}-q t$ or, equivalently, $(q, T(q)) \in \Sigma_{2}\left(\phi,-\log \left|f^{\prime}\right|_{\tau}\right)$.

Proof. The function $T \mapsto \mathrm{P}\left(\phi_{q, T}\right), T>\frac{\rho}{\hat{\tau}}-q t$, being differentiable (Lemma 7.5) with

$$
\frac{\partial \mathrm{P}\left(\phi_{q, T}\right)}{\partial T}=-\int \log \left|f^{\prime}\right|_{\tau} d \mu_{q \phi-t \log \left|f^{\prime}\right|_{\tau}} \leq-\log \gamma<0
$$

(Theorem 7.14) we conclude that this function is strictly decreasing with

$$
\lim _{T \rightarrow \infty} \mathrm{P}\left(\phi_{q, T}\right)=-\infty
$$

It remains to show that $\mathrm{P}\left(\phi_{q, T}\right)>0$ for some $T \geq \frac{\rho}{\hat{\tau}}-q t$ because then the function $T \mapsto \mathrm{P}\left(\phi_{q, T}\right)$ has exactly one zero

$$
T(q)>\frac{\rho}{\hat{\tau}}-q t .
$$

In order to do so, set $s=T+q t$ and $\psi_{s}=-s \log \left|f^{\prime}\right|_{\tau}+q h=\phi_{q, T}$. If $f$ has a pole then, by the assumption made in Definition 2.5. it also has a pole $b$ of maximal multiplicity $q<\infty$ and $\underline{\alpha}_{2}=1+\frac{1}{q}$. The divergence type assumption (2.6) and a result in My3 (more precisely the Remark 3.2 in that paper) shows that $\operatorname{HD}\left(\mathcal{J}_{r}(f)\right)>\frac{\rho}{\alpha}$ which implies that

$$
\mathrm{P}\left(\psi_{s_{0}}\right)>0, s_{0}=\frac{\rho}{\hat{\tau}},
$$

provided $\tau$ satisfies

$$
\frac{\rho}{\alpha}<\frac{\rho}{\hat{\tau}}<\operatorname{HD}\left(\mathcal{J}_{r}(f)\right)
$$

So, let finally $f$ be entire. Notice first that, with the balanced growth condition and the fact that $\alpha_{2}$ is a constant function (Definition 2.5), the calculations leading to (5.4) give the following lower estimate.

$$
\mathcal{L}_{\phi_{q, T}} \mathbb{1}(w)=\sum_{z \in f^{-1}(w)}\left|f^{\prime}(z)\right|_{\sigma}^{-s} e^{q h(z)} \geq \frac{\kappa^{-s} e^{-q\|h\|_{\infty}}}{|w|^{\left(\alpha_{2}-\tau\right) s}} \sum_{z \in f^{-1}(w)}|z|^{-\hat{\tau} s}, w \in \mathcal{J}(f),
$$


for all $s>s_{0}=\rho / \hat{\tau}$. Denote now for every $R>0$

$$
\Sigma^{R}(u, a)=\sum_{z \in f^{-1}(a) \cap D(0, R)}|z|^{-u} \quad, a \in \mathcal{J}(f) \text { and } u \geq \rho .
$$

Claim: There exists $R>T$ such that

$$
\Sigma^{R}(\rho, a) \geq A=8 \kappa^{s_{0}} e^{q\|h\|_{\infty}} \quad \text { for all } a \in \mathcal{J}(f) \cap D(0, R) .
$$

Suppose this claim holds. Let $\tau_{0}<\alpha_{2}$ be such that $R^{\left(\alpha_{2}-\tau_{0}\right) s_{0}} \leq 2$. Then for every $\tau \in\left(\tau_{0}, \alpha_{2}\right)$ and every $s>s_{0}$ sufficiently close to $s_{0}$ we get

$$
\mathcal{L}_{\phi_{q, T}} \mathbb{1}_{D(0, R)}(w) \geq 2 \quad \text { for every } w \in \mathcal{J}(f) \cap D(0, R),
$$

from which $\mathrm{P}\left(\psi_{s_{0}}\right)>0$ follows.

It remains to prove the claim. Let $R>T$ and let $a \in D(0, R) \cap \mathcal{J}(f)$. We get precisely in the same way as in (3.9) that

$$
\Sigma^{R}(\rho, a) \geq \rho^{2} \int_{0}^{R} \frac{N(t, a)}{t^{\rho+1}} d t \geq \int_{\log |a|}^{R} \frac{N(t, a)}{t^{\rho+1}} d t .
$$

From the sharp form of the SMT (Lemma 3.4), it follows that

$$
\begin{aligned}
\Sigma^{R}(\rho, a) & \geq \rho^{2} \int_{\log |a|-\Delta}^{R-\Delta} \frac{N(r+\Delta, a)}{r^{\rho+1}}\left(\frac{r}{r+\Delta}\right)^{\rho+1} d r \asymp \int_{\log |a|-\Delta}^{R-\Delta} \frac{4 N(r+\Delta, a)}{r^{\rho+1}} d r \\
& \geq \int_{\log |a|-\Delta}^{R-\Delta} \frac{T(r)}{r^{\rho+1}} d r-C_{1}-C_{2}(\log |a|)^{1-\rho}
\end{aligned}
$$

for some constants $C_{1}, C_{2}>0$. If the order $\rho \geq 1$ then $(\log |a|)^{1-\rho}$ is bounded above. Consequently there are $C_{3}, C_{4}>0$ such that

$$
\Sigma^{R}(\rho, a) \geq C_{3} \int_{\log R-\Delta}^{R-\Delta} \frac{T(r)}{r^{\rho+1}} d r-C_{4} .
$$

In the case when $0<\rho<1$, we have $(\log |a|)^{1-\rho} \leq(\log R)^{1-\rho} \asymp(\log (R-\Delta))^{1-\rho}$. Therefore

$$
\Sigma^{R}(\rho, a) \geq C_{3} \int_{\log R-\Delta}^{R-\Delta} \frac{T(r)}{r^{\rho+1}} d r-C_{1}-C_{5}(\log (R-\Delta))^{1-\rho}
$$

for some $C_{5}>0$. The assertion follows now from the assumption (2.7).

If $q=0$ then $\phi_{q, T}=-T \log \left|f^{\prime}\right|_{\tau}$ is what we called a geometric potential, i.e. no additional Hölder function is involved. The following result justifies particularly well this denomination. It gives in the same time a geometric meaning to this unique zero of the pressure function. This Bowen's Formula has been obtained in MyU2.

Theorem 8.3 (Bowen's Formula). With the assumptions of the above Lemma, the only zero $h$ of $T \mapsto \mathrm{P}\left(-T \log \left|f^{\prime}\right|_{\tau}\right)$ is

$$
h=\mathrm{HD}\left(\mathcal{J}_{r}(f)\right) .
$$


Proof. Denote $\mu_{h}=\mu_{-h \log \left|f^{\prime}\right|_{\tau}}$ and $m_{h}=m_{-h} \log \left|f^{\prime}\right|_{\tau}$. First of all, the Variational Principle (Theorem 6.25) gives

$$
0=\mathrm{P}(h)=h_{\mu_{h}}-h \chi_{\mu_{h}} .
$$

Consequently we get from the Volume Lemma (Theorem 8.1) and the fact that $\mu_{h}\left(\mathcal{J}_{r}(f)\right)=1$ that

$$
h=\frac{h_{\mu_{h}}}{\chi_{\mu_{h}}}=\operatorname{HD}\left(\mu_{h}\right) \leq \operatorname{HD}\left(\mathcal{J}_{r}(f)\right) .
$$

It remains to establish the opposite inequality $\operatorname{HD}\left(\mathcal{J}_{r}(f)\right) \leq h$. Since $\mu_{h}$ is an ergodic measure there is $M_{0}>0$ so large that $\mu_{h}\left(J_{r, M}(f)\right)=1$ for every $M \geq M_{0}$ where

$$
J_{r, M}(f)=\left\{z \in \mathcal{J}(f): \liminf _{n \rightarrow \infty}\left|f^{n}(z)\right|<M\right\} .
$$

Consequently $m_{h}\left(J_{r, M}(f)\right)=1$. Since $\mathcal{J}(f) \cap \bar{D}(0, M)$ is a compact set,

$$
Q_{M}:=\inf \left\{m_{h}(D(w, \delta): w \in \mathcal{J}(f) \cap D(0, M))\right\}>0 .
$$

Now, fix $z \in J_{r, M}(f)$ and consider an arbitrary integer $n \geq 0$ such that $f^{n}(z) \in$ $D(0, M)$. It follows from conformality of the measure $m_{h}$, Koebe's Distortion Theorem and the fact that $\mathrm{P}(h)=0$ that

$$
\begin{gathered}
m_{h}\left(D\left(z, \delta K\left|\left(f^{n}\right)^{\prime}(z)\right|^{-1}\right)\right) \succeq\left|\left(f^{n}\right)^{\prime}(z)\right|_{\tau}^{-h} m_{h}\left(D\left(f^{n}(z), \delta\right)\right) \\
\geq Q_{M}\left|\left(f^{n}\right)^{\prime}(z)\right|^{-h}\left(\frac{\left|f^{n}(z)\right|}{|z|}\right)^{h} .
\end{gathered}
$$

Recall that $D(0, T) \cap \mathcal{J}(f)=\emptyset$. Therefore $m_{h}\left(D\left(z, \delta K\left|\left(f^{n}\right)^{\prime}(z)\right|^{-1}\right)\right) \succeq\left|\left(f^{n}\right)^{\prime}(z)\right|^{-h}$. Thus, there exists $c>0$ such that for every $z \in J_{r, M}(f)$

$$
\limsup _{r \rightarrow 0} \frac{m_{h}(D(z, r))}{r^{h}} \geq \limsup _{n \rightarrow \infty} \frac{m_{h}\left(D\left(z, K \delta\left|\left(f^{n}\right)^{\prime}(z)\right|^{-1}\right)\right)}{\left(K \delta\left|\left(f^{n}\right)^{\prime}(z)\right|^{-1}\right)^{h}} \geq c .
$$

This implies that $\operatorname{HD}\left(J_{r, M}(f)\right) \leq h$ for every $M \geq M_{0}$. Consequently $\operatorname{HD}\left(\mathcal{J}_{r}(f)\right) \leq$ $h$ and the proof is complete.

As a first application we can now complete the discussion on the different radial sets $\mathcal{J}_{r}(f)$ and $\Lambda_{f}$ (see Remark 4.1).

Proposition 8.4. Assuming again the assumptions of Lemma 8.2 we have that

$$
\operatorname{HD}\left(\mathcal{J}_{r}(f)\right)=\operatorname{HD}\left(\Lambda_{f}\right) .
$$

Proof. Let $h=\operatorname{HD}\left(\mathcal{J}_{r}(f)\right)$ the zero of the pressure function. Since $\mathcal{J}_{r}(f) \subset$ $\Lambda_{f}$ we have $h \leq \operatorname{HD}\left(\Lambda_{f}\right)$ and it suffices to show that

$$
\operatorname{HD}\left(\Lambda_{f} \backslash \mathcal{J}_{r}(f)\right)=\operatorname{HD}\left(\Lambda_{f} \cap I_{\infty}(f)\right) \leq h .
$$

Consider again the $h$-conformal measure $m_{h}=m_{-h \log \left|f^{\prime}\right|_{\tau}}$. We have to work with the spherical metric. So let $\nu=|z|^{h(\tau-2)} m_{h}$ be the spherical $h$-conformal measure. Notice that $\nu$ is a finite measure since $\tau \leq 2$.

If $z \in \Lambda_{f} \cap I_{\infty}(f)$, then there is $\delta>0$ and $n_{j} \rightarrow \infty$ such that $f^{n_{j}}: U_{j} \rightarrow$ $D_{2}(\infty, \delta)$ is conformal with bounded distortion where $D_{2}(\infty, \delta)$ signifies the spherical disk centered at $\infty$ with radius $\delta$ and where $U_{j}$ is the component of $f^{-1}\left(D_{2}(\infty, \delta)\right)$ that contains $z$. Conformality and bounded distortion of $f^{n_{j}}$ on $U_{j}$ implies that

$$
U_{j} \asymp D_{2}\left(z,\left|\left(f^{n_{j}}\right)^{\prime}\right|_{2}^{-1} \delta\right) \quad \text { and } \quad \nu\left(U_{j}\right) \asymp\left|\left(f^{n_{j}}\right)^{\prime}\right|_{2}^{-h}
$$


from which follows that $\nu\left(D_{2}\left(z, r_{j}\right)\right) \asymp r_{j}^{h}$ with $r_{j}=\left|\left(f^{n_{j}}\right)^{\prime}\right|_{2}^{-1} \delta$. Inequality 8.13) follows now with standard arguments since we know that $\nu\left(I_{\infty}(f)\right)=0$ (Proposition 5.21).

Let us now come to general $q \in \mathbb{R}$ and potentials $\phi_{q, T}$. In the following definition it is important to normalize the potentials. Subtracting $\mathrm{P}(\phi)$ from $\phi$, we can assume without loss of generality that

$$
\mathrm{P}(\phi)=0
$$

and call $\phi$ normalized.

Definition 8.5. Suppose that $\phi$ is normalized and set again $\phi_{q, T}=-T \log \left|f^{\prime}\right|_{\tau}+$ $q \phi$. The temperature function is

$$
q \in \mathbb{R} \mapsto T(q) \in\left(\frac{\rho}{\hat{\tau}}-q t, \infty\right)
$$

where $T(q)$ is the only zero of $T \mapsto \mathrm{P}\left(\phi_{q, T}\right)$.

Bowen's Formula can now be reformulated as $T(0)=h=\mathrm{HD}\left(\mathcal{J}_{r}(f)\right)$.

Theorem 8.6. The temperature function $q \mapsto T(q)$ is real analytic with $T^{\prime}(q)<0$ and $T^{\prime \prime}(q) \geq 0$. In addition the following are equivalent:

(1) $T^{\prime \prime}$ vanishes in one point.

(2) $T^{\prime \prime}$ vanishes at all points.

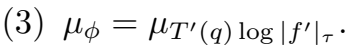

(4) $\phi$ and $T^{\prime}(q) \log \left|f^{\prime}\right|_{\tau}$ are cohomologous modulo a constant in the class of all Hölder continuous functions.

If one of these properties holds then $-T^{\prime}(q)$ is constant equal to the only zero of the pressure function $t \mapsto \mathrm{P}\left(-t \log \left|f^{\prime}\right|_{\tau}\right)$ which is $h=\operatorname{HD}\left(\mathcal{J}_{r}(f)\right)$.

We put in the following

$$
m_{q}=m_{q \phi-T(q) \log \left|f^{\prime}\right|_{\tau}}=m_{\phi_{q, T(q)}} \quad, \quad \mu_{q}=\mu_{\phi_{q, T(q)}} .
$$

Proof. By assumption, the potential $\phi$ is normalized, i.e. $\mathrm{P}(\phi)=0$. Since, by Theorem 7.14 and the expanding property of $f$,

$$
\frac{\partial \mathrm{P}}{\partial t}=-\int \log \left|f^{\prime}\right|_{\tau} d \mu_{q} \leq-\log \gamma<0,
$$

applying Lemma 7.6, we infer that the function $q \mapsto T(q), q \in \mathbb{R}$, is real-analytic. Differentiating the equation $\mathrm{P}\left(\phi_{q, T}\right)=0$ and using Theorem 7.14 again, we obtain

$$
0=\frac{\partial \mathrm{P}}{\partial t} T^{\prime}(q)+\frac{\partial \mathrm{P}}{\partial q}=-T^{\prime}(q) \int \log \left|f^{\prime}\right|_{\tau} d \mu_{q}+\int \phi d \mu_{q} .
$$

Therefore

$$
T^{\prime}(q)=\frac{\int \phi d \mu_{q}}{\int \log \left|f^{\prime}\right|_{\tau} d \mu_{q}}<0
$$


The equality $T(0)=h=\operatorname{HD}\left(J_{r}(f)\right)$ is just Bowen's Formula. Let us now show that the function $T: \mathbb{R} \rightarrow \mathbb{R}$ is convex, i.e. that $T^{\prime \prime}(q) \geq 0$ for all $q \in \mathbb{R}$. Differentiating the first part of (8.14), we obtain

$$
\begin{aligned}
T^{\prime \prime}(q) & =-\frac{T^{\prime}(q)^{2} \frac{\partial^{2} \mathrm{P}}{\partial t^{2}}+2 T^{\prime}(q) \frac{\partial^{2} \mathrm{P}\left(\phi_{q, T}\right)}{\partial q \partial t}+\frac{\partial^{2} \mathrm{P}}{\partial q^{2}}}{\frac{\partial \mathrm{P}}{\partial t}} \\
& =\frac{T^{\prime}(q)^{2} \frac{\partial^{2} \mathrm{P}}{\partial t^{2}}+2 T^{\prime}(q) \frac{\partial^{2} \mathrm{P}}{\partial q \partial t}+\frac{\partial^{2} \mathrm{P}}{\partial q^{2}}}{\chi_{\mu_{q}}},
\end{aligned}
$$

where $\chi_{\mu_{q}}=\int \log \left|f^{\prime}\right| d \mu_{q}$ is the characteristic Lyapunov exponent of the measure $\mu_{q}$. Invoking Theorem 7.15 we see that

$$
\frac{\partial^{2} \mathrm{P}}{\partial t^{2}}=\hat{\sigma}_{\mu_{q}}^{2}\left(-\log \left|f^{\prime}\right|_{\tau}\right), \frac{\partial^{2} \mathrm{P}}{\partial q \partial t}=\hat{\sigma}_{\mu_{q}}^{2}\left(-\log \left|f^{\prime}\right|_{\tau}, \phi\right) \frac{\partial^{2} \mathrm{P}}{\partial q^{2}}=\hat{\sigma}_{\mu_{q}}^{2}(\phi),
$$

Using these formulas a straightforward but lengthy calculation, based on Theorem 7.15 shows that

$$
\chi_{\mu_{q}} T^{\prime \prime}(q)=T^{\prime}(q)^{2} \frac{\partial^{2} \mathrm{P}}{\partial t^{2}}+2 T^{\prime}(q) \frac{\partial^{2} \mathrm{P}}{\partial q \partial t}+\frac{\partial^{2} \mathrm{P}}{\partial q^{2}}=\hat{\sigma}_{\mu_{q}}^{2}\left(-T^{\prime}(q) \log \left|f^{\prime}\right|_{\tau}+\phi\right) .
$$

Since $\hat{\sigma}_{\mu_{q}}^{2}\left(-T^{\prime}(q) \log \left|f^{\prime}\right|_{\tau}+\phi\right) \geq 0$ (and $\chi_{\mu_{q}}>0$ ), we conclude that $T^{\prime \prime}(q) \geq 0$.

Passing to the proof of the the equivalence of the assertions (1) to (4), notice that, in view of (8.16) and (8.17),

$$
T^{\prime \prime}(q)=0 \text { if and only if } \hat{\sigma}_{\mu_{q}}^{2}\left(-T^{\prime}(q) \log \left|f^{\prime}\right|_{\tau}+\phi\right)=0 \text {, }
$$

which, in view of Proposition 6.21, implies that the function $-T^{\prime}(q) \log \left|f^{\prime}\right|_{\tau}+\phi$ is cohomologous to a constant, say $a$, in the class of Hölder continuous functions on $\mathcal{J}(f)$. But this can only happen if $-T^{\prime}(q) \log \left|f^{\prime}\right|_{\tau}+\phi=h$ is a 0 -tame potential (cf. Theorem 6.20). In particular, this function is bounded and cohomologous to a constant in which case we have the equality

$$
\hat{\sigma}^{2}(h)=\sigma^{2}(h)
$$

in the CLT. Therefore $T^{\prime \prime}(q)=0$ for all $q \in \mathbb{R}$. Finally, the equivalence between (3) and (4) is given in Theorem 6.19

\subsection{Multifractal analysis}

Recall that we investigate here the multifractal spectrum $\mathcal{F}_{\phi}(\alpha)=\operatorname{HD}\left(D_{\phi}(\alpha)\right)$ where $D_{\phi}(\alpha)=\left\{z \in \mathcal{J}_{r}(f) ; d_{\mu_{\phi}}(z)=\alpha\right\}$. One of our goals is to establish that the multifractal formalism is satisfied meaning that $\mathcal{F}_{\phi}$ and the temperature function build a Legendre transform pair. If $k$ is a strictly convex map on an interval $I$, then the Legendre transform of $k$ is the function $h$ of the new variable $p=k^{\prime}(x)$ defined by

$$
h(p)=\max _{I}\{p x-k(x)\}
$$

everywhere where this maximum exists. It can be proved that the domain of $g$ is either a point, an interval or a semi-line. It is also easy to show that $g$ is strictly convex and that the Legendre transform is involutive. We then say that the functions $k$ and $h$ form a Legendre transform pair. The following fact gives a useful characterization of a Legendre transform pair (see [Ro]). 
Fact 8.7. Two strictly convex functions $k$ and $g$ form a Legendre transform pair if and only if $g(p)=p x-k(x)$ with $p=k^{\prime}(x)$.

Theorem 8.8. Let $f: \mathbb{C} \rightarrow \hat{\mathbb{C}}$ be a divergence type and dynamically regular meromorphic function of order $\rho>0$ and let $\varphi=-t \log \left|f^{\prime}\right|_{\tau}+h$ be a tame potential. Then the following statements are true.

(1) For every $q \in \mathbb{R}$,

$$
\mathcal{F}_{\phi}(\alpha)=\alpha q+T(q) \text { with } \alpha=-T^{\prime}(q) .
$$

If $\mu_{\phi} \neq \mu_{-h \log \left|f^{\prime}\right|_{\tau}}$ then the functions $\alpha \mapsto-\mathcal{F}_{\phi}(-\alpha)$ and $T(q)$ form a Legendre transform pair.

(2) The function $\alpha \mapsto \mathcal{F}_{\phi}(\alpha)$ is real-analytic throughout its whole domain $\left(\alpha_{1}, \alpha_{2}\right) \subset[0, \infty)$.

(3) $\alpha_{1}=\alpha_{2}$ if and only if $\mu_{\phi}=\mu_{-h \log \left|f^{\prime}\right|_{\tau}}$ with $h=\operatorname{HD}\left(\mathcal{J}_{r}(f)\right.$ ) (and then $\left.\alpha_{1}=\alpha_{2}=h\right)$.

In order to prove this result we need the following auxiliary considerations. First we define a set $\mathcal{J}_{r r}(f) \subset \mathcal{J}_{r}(f)$ suitable for multifractal analysis on balls. Given $R>0$ and a point $z \in \mathcal{J}_{r}(f)$ let $n_{j}=n_{j}(z, R)$ be the sequence of consecutive visits of the point $z$ to $D(0, R)$ under the action of $f$, i.e. this sequence is strictly increasing (perhaps finite, perhaps empty) with $f^{n_{j}}(z) \in D(0, R)$ for all $j \geq 1$ and $f^{n}(z) \notin D(0, R)$ for all $n_{j}<n<n_{j+1}$. Let $M_{R}$ be the set of points $z \in \mathcal{J}_{r}(f)$ such that

$$
\lim _{j \rightarrow \infty} \frac{\log \left|\left(f^{n_{j+1}-n_{j}}\right)^{\prime}\left(f^{n_{j}}(z)\right)\right|}{\log \left|\left(f^{n_{j}}\right)^{\prime}(z)\right|}=0 \text { and } \lim _{j \rightarrow \infty} \frac{n_{j+1}}{n_{j}}=1
$$

where $n_{j}=n_{j}(z, R)$. Denote then

$$
\mathcal{J}_{r r}(f)=\bigcup_{R>0} M_{R}
$$

Observe that if $z \in M_{R}$ then, for every $p \geq 1$,

$$
\lim _{j \rightarrow \infty} \frac{\log \left|\left(f^{n_{j+p}-n_{j}}\right)^{\prime}\left(f^{n_{j}}(z)\right)\right|}{\log \left|\left(f^{n_{j}}\right)^{\prime}(z)\right|}=0 \text { and } \lim _{j \rightarrow \infty} \frac{n_{j+p}}{n_{j}}=1 .
$$

Now let us record the fact that this set $\mathcal{J}_{r r}(f)$ is dynamically significant.

Proposition 8.9. If $\mu$ is a Borel probability $f$-invariant ergodic measure on $\mathcal{J}(f)$ with finite Lyapunov exponent $\chi_{\mu}$ (which is in particular the case for every Gibbs state $\mu_{\phi}$ with $\phi$ a tame potential $)$, then $\mu\left(\mathcal{J}_{r r}(f)\right)=1$.

Proof. Let $R>0$ such that $D(0, R) \cap \mathcal{J}(f) \neq \emptyset$. We keep the notation $n_{j}$ for $n_{j}(z, R)$. Since

and since by Birkhoff's Ergodic Theorem

$$
\left(f^{n_{j+1}-n_{j}}\right)^{\prime}\left(f^{n_{j}}(z)\right)=\frac{\left(f^{n_{j+1}}\right)^{\prime}(z)}{\left(f^{n_{j}}\right)^{\prime}(z)}
$$

$$
\lim _{j \rightarrow \infty} \frac{n_{j+1}}{n_{j}}=1
$$

for $\mu$-a.e. $z \in \mathcal{J}_{r}(f)$, the proof is concluded by applying Birkhoff's Ergodic Theorem to the integrable function $\log \left|f^{\prime}\right|$. 
Given a real number $\alpha \geq 0$, we define the following set.

$$
\mathcal{K}_{\phi}(\alpha)=\left\{z \in J_{r}(f): \lim _{n \rightarrow \infty} \frac{\mathrm{P}(\phi) n-S_{n} \phi(z)}{\log \left|\left(f^{n}\right)^{\prime}(z)\right|}=\alpha\right\} .
$$

Proposition 8.10. For every $\alpha \geq 0$, we have that

$$
\mathcal{K}_{\phi}(\alpha) \cap J_{r r}(f) \subset D_{\phi}(\alpha) .
$$

Proof. We are to prove that if $z \in J_{r r}(f)$, then

$$
\lim _{j \rightarrow \infty} \frac{\mathrm{P}(\phi) j-S_{j} \phi(z)}{\log \left|\left(f^{j}\right)^{\prime}(z)\right|}=\alpha \Longrightarrow \lim _{r \rightarrow 0} \frac{\log \mu_{\phi}(D(z, r))}{\log r}=\alpha .
$$

And indeed, take $z \in J_{r r}(f)$ and assume that the left-hand side limit of (8.18) is equal to $\alpha$. Let $R>0$ such that $z \in M_{R}$ and denote again $n_{j}=n_{j}(z, R)$. Fix $r \in(0,1)$ small enough and let $j=j(r) \geq 1$ be the largest integer such that

$$
r\left|\left(f^{n_{j}}\right)^{\prime}(z)\right| \leq \delta / 4
$$

Then

$$
r\left|\left(f^{n_{j+1}}\right)^{\prime}(z)\right|>\delta / 4,
$$

It follows from (8.20) and Koebe's Distortion Theorem that

$$
f_{z}^{-n_{j+1}}\left(D\left(f^{n_{j+1}}(z),(4 K)^{-1} \delta\right)\right) \subset D(z, r)
$$

and from (8.20) along with Koebe's $\frac{1}{4}$-Distortion Theorem, that

$$
f_{z}^{-n_{j}}\left(D\left(f^{n_{j}}(z), \delta\right)\right) \supset D(z, r) .
$$

Put

$$
\psi=\phi-\mathrm{P}(\phi) .
$$

Applying Lemma 5.20 we therefore get that

$$
\begin{aligned}
m_{\phi}(D(z, r)) & \leq m_{\phi}\left(f_{z}^{-n_{j}}\left(D\left(f^{n_{j}}(z), \delta\right)\right)\right) \leq C_{\phi} \exp \left(S_{n_{j}} \psi(z)\right) m_{\phi}\left(D\left(f^{n_{j}}(z), \delta\right)\right) \\
& \leq C_{\phi} \exp \left(S_{n_{j}} \psi(z)\right)
\end{aligned}
$$

and

$$
\begin{aligned}
m_{\phi}(D(z, r)) & \geq m_{\phi}\left(f_{z}^{-n_{j+1}}\left(D\left(f^{n_{j+1}}(z),(4 K)^{-1} \delta\right)\right)\right) \\
& \geq C_{\phi}^{-1} \exp \left(S_{n_{j+1}} \psi(z)\right) m_{\phi}\left(D\left(f^{n_{j+1}}(z),(4 K)^{-1} \delta\right)\right) \\
& \geq T C_{\phi}^{-1} \exp \left(S_{n_{j+1}} \psi(z)\right),
\end{aligned}
$$

where $T=\inf \left\{m_{\phi}\left(D\left(w,(4 K)^{-1} \delta\right): w \in \mathcal{J}(f) \cap D(0, R)\right\}>0\right.$. Using these two estimates and both (8.19) and (8.20), we obtain

$$
\begin{aligned}
\frac{\log \left(m_{\phi}(D(z, r))\right)}{\log r} & \geq \frac{\log C_{\phi}+S_{n_{j}} \psi(z)}{\log r} \geq \frac{\log C_{\phi}+S_{n_{j}} \psi(z)}{\log (\delta / 4)-\log \left|\left(f^{n_{j+1}}(z)\right)^{\prime}(z)\right|} \\
& =\frac{\log C_{\phi}+S_{n_{j}} \psi(z)}{\log (\delta / 4)-\log \left|\left(f^{n_{j}}(z)\right)^{\prime}(z)\right|-\log \left|\left(f^{n_{j+1}-n_{j}}\right)^{\prime}\left(f^{n_{j}}(z)\right)\right|} .
\end{aligned}
$$


and

$(8.22)$

$$
\begin{aligned}
\frac{\log \left(m_{\phi}(D(z, r))\right)}{\log r} & \leq \frac{\log T-\log C_{\phi}+S_{n_{j+1}} \psi(z)}{\log r} \leq \frac{\log T-\log C_{\phi}+S_{n_{j+1}} \psi(z)}{\log (\delta / 4)-\log \left|\left(f^{n_{j}}(z)\right)^{\prime}(z)\right|} \\
& =\frac{\log T-\log C_{\phi}+S_{n_{j+1}} \psi(z)}{\log (\delta / 4)-\log \left|\left(f^{n_{j+1}}(z)\right)^{\prime}(z)\right|+\log \left|\left(f^{n_{j+1}-n_{j}}\right)^{\prime}\left(f^{n_{j}}(z)\right)\right|}
\end{aligned}
$$

Dividing the numerators and the denominators of the right-hand sides of (8.21) and (8.22) respectively by $\log \left|\left(f^{n_{j}}(z)\right)^{\prime}(z)\right|$ and $\log \left|\left(f^{n_{j+1}}(z)\right)^{\prime}(z)\right|$, and noting also that $\lim _{r \rightarrow 0} n_{j(r)}=\lim _{r \rightarrow 0} n_{j(r)+1}=+\infty$, we thus get that

$$
\liminf _{r \rightarrow 0} \frac{\log \left(m_{\phi}(D(z, r))\right)}{\log r} \geq \alpha \text { and } \limsup _{r \rightarrow 0} \frac{\log \left(m_{\phi}(D(z, r))\right)}{\log r} \leq \alpha .
$$

Since $\lim _{r \rightarrow 0} \frac{\log \left(\mu_{\phi}(D(z, r))\right)}{\log r}=\lim _{r \rightarrow 0} \frac{\log \left(m_{\phi}(D(z, r))\right)}{\log r}$, we are done.

Proof of Theorem 8.8. Remember that

$$
m_{q}=m_{q \phi-T(q) \log \left|f^{\prime}\right|_{\tau}}=m_{\phi_{q, T(q)}} \quad, \quad \mu_{q}=\mu_{\phi_{q, T(q)}}
$$

and $\alpha=-T^{\prime}(q)$.

In order to prove (1), we first give the estimate of the function $\mathcal{F}_{\phi}(\alpha)$ from below. By Birkhoff's Ergodic Theorem and Proposition 8.9 there exists a Borel set $X \subset J_{r r}(f)$ such that $\mu_{q}(X)=1$, and such that for every $x \in X$,

$$
\lim _{n \rightarrow \infty} \frac{1}{n} \log \left|\left(f^{n}\right)^{\prime}(x)\right|=\int \log \left|f^{\prime}\right| d \mu_{q} \text { and } \lim _{n \rightarrow \infty} \frac{1}{n} S_{n} \phi_{q, T(q)}(x)=\int \phi_{q, T(q)} d \mu_{q} .
$$

Hence, using (8.15), we obtain for every $x \in X$

$$
\lim _{n \rightarrow \infty} \frac{-S_{n} \phi_{q, T(q)}(x)}{\log \left|\left(f^{n}\right)^{\prime}(x)\right|}=-\frac{\int \phi_{q, T(q)} d \mu_{q}}{\int \log \left|f^{\prime}\right| d \mu_{q}}=\alpha,
$$

In other words, $X \subset \mathcal{J}_{r r}(f) \cap \mathcal{K}_{\phi_{q, T(q)}}(\alpha)$ and hence, by Proposition 8.10, $X \subset$ $D_{\phi}(\alpha)$.

Thus, the Volume Lemma (Theorem 8.1), the fact that $\mathrm{P}\left(\phi_{q, T(q)}\right)=0$, the Variational Principle (Theorem 6.25) and (8.15) imply that

$$
\begin{aligned}
\mathcal{F}_{\phi}(\alpha) & =\operatorname{HD}\left(D_{\phi}(\alpha)\right) \geq \operatorname{HD}(X) \geq \operatorname{HD}\left(\mu_{q}\right)=\frac{\mathrm{h}_{\mu_{q}}(f)}{\chi_{\mu_{q}}} \\
& =\frac{T(q) \chi_{\mu_{q}}-q \int \phi d \mu_{q}}{\chi_{\mu_{q}}}=T(q)-q \frac{\int \phi d \mu_{q}}{\chi_{\mu_{q}}}=T(q)-q T^{\prime}(q) .
\end{aligned}
$$

This gives the required lower bound for $\mathcal{F}_{\phi}$. For the upper bound of $\mathcal{F}_{\phi}$ let us fix an element $x \in \mathcal{D}_{\phi}(\alpha)$. Since $x \in J_{r}(f)$, there exist $M>0$ and an unbounded increasing sequence $\left\{k_{n}\right\}_{n=1}^{\infty}$ such that $\left|f^{k_{n}}(x)\right| \leq M$ for all $n \geq 1$. The estimate (8.12) gives us that

$$
m_{q}\left(D\left(x, \delta\left|\left(f^{k_{n}}\right)^{\prime}(x)\right|^{-1}\right)\right) \geq C \exp \left(S_{k_{n}}\left(-T(q) \log \left|f^{\prime}\right|+q \phi\right)(x)\right)
$$


with some constant $C$ independent of $x$ and $n$. Hence,

$$
\begin{aligned}
\liminf _{r \rightarrow 0} \frac{\log m_{q}(D(x, r))}{\log r} & \leq \liminf _{n \rightarrow \infty} \frac{\log m_{q}\left(D\left(x, \delta\left|\left(f^{k_{n}}\right)^{\prime}(x)\right|^{-1}\right)\right)}{\log \left(\delta\left|\left(f^{k_{n}}\right)^{\prime}(x)\right|^{-1}\right)} \\
& \leq \lim _{n \rightarrow \infty} \frac{-T(q) \log \left|\left(f^{k_{n}}\right)^{\prime}(x)\right|+q S_{k_{n}} \phi(x)}{-\log \left|\left(f^{k_{n}}\right)^{\prime}(x)\right|} .
\end{aligned}
$$

Take now the measure $m_{\phi}$. By the same arguments as above one get's from conformality of this measure that

$$
m_{\phi}\left(D\left(x, \delta\left|\left(f^{k_{n}}\right)^{\prime}(x)\right|^{-1}\right)\right) \leq c \exp \left(S_{k_{n}}(\phi)(x)\right) .
$$

Since $x \in \mathcal{D}_{\phi}(\alpha)$ we get

$$
\alpha=\lim _{k \rightarrow \infty} \frac{\log m_{\phi}\left(D\left(x, \delta\left|\left(f^{k_{n}}\right)^{\prime}(x)\right|^{-1}\right)\right)}{\log \left(\delta\left|\left(f^{k_{n}}\right)^{\prime}(x)\right|^{-1}\right)} \geq \lim _{k \rightarrow \infty} \frac{S_{k_{n}}(\phi)(x)}{\log \left(\delta\left|\left(f^{k_{n}}\right)^{\prime}(x)\right|^{-1}\right)} .
$$

Together with (8.24) we finally have

$$
\liminf _{r \rightarrow 0} \frac{\log m_{q}(D(x, r))}{\log r} \leq T(q)+q \alpha .
$$

So the proof of item (1) is complete.

The assertion (2) results from (1) together with Theorem 8.6. The same Theorem 8.6 yields also $(3)$. 


\section{CHAPTER 9}

\section{Multifractal Analysis of Analytic Families of Dynamically Regular Functions}

Fixing a uniformly balanced bounded deformation family of divergence type dynamically regular transcendental functions we perform the multifractal analysis for potentials of the form

$$
-t \log \left|f_{\lambda}^{\prime}\right|_{\sigma}+h
$$

where $h$ is a real-valued bounded harmonic function defined on an open neighborhood of the Julia set of a fixed member of $\Lambda$. We show that the multifractal function $\mathcal{F}_{\phi}(\lambda, \alpha)$ depends real analytically not only on the multifractal parameter $\alpha$ but also on $\lambda$. As a by-product of our considerations in this chapter, we reproduce from MyU2, providing all details, real-analytic dependence of $\operatorname{HD}\left(J_{r}\left(f_{\lambda}\right)\right)$ on $\lambda$ (Theorem 9.11). At the end of this chapter we provide a fairly easy sufficient condition for the multifractal spectrum not to degenerate.

\subsection{Extensions of Harmonic Functions}

Fix $d \geq 1$. Embed $\mathbb{C}^{d}$ into $\mathbb{C}^{2 d}$ by the formula

$$
\left(x_{1}+i y_{1}, x_{2}+i y_{2}, \ldots, x_{d}+i y_{d}\right) \mapsto\left(x_{1}, y_{1}, x_{2}, y_{2}, \ldots, x_{d}, y_{d}\right) .
$$

For every $z \in \mathbb{C}^{d}$ and every $r>0$ denote by $D_{d}(z, r)$ the $d$-dimensional polydisk in $\mathbb{C}^{d}$ centered at $z$ and with "radius" $r$. By $B(X, R)$ we will denote the $R$-neighbourhood of the set $X$. We will need the following lemma, which is of general character independent of dynamics.

Lemma 9.1. For every $M \geq 0$, for every $R>0$, for every $\lambda^{0} \in \mathbb{C}^{d}$, and for every analytic function $\psi: D_{d}\left(\lambda^{0}, R\right) \rightarrow \mathbb{C}$ bounded in modulus by $M$ there exists an analytic function $\Re \psi: D_{2 d}\left(\lambda^{0}, R / 4\right) \rightarrow \mathbb{C}$ that is bounded in modulus by $4^{d} M$ and whose restriction to the polydisk $D_{d}\left(\lambda^{0}, R / 4\right)$ coincides with $\operatorname{Re} \psi$, the real part of $\psi$.

Proof. Denote by $\mathbb{N}_{0}$ the set of all non-negative integers. Write the analytic function $\psi: D_{d}\left(\lambda^{0}, R\right) \rightarrow \mathbb{C}$ in the form of its Taylor series expansion

$$
\psi\left(\lambda_{1}, \lambda_{2}, \ldots, \lambda_{d}\right)=\sum_{\alpha \in \mathbb{N}_{0}^{d}} a_{\alpha}\left(\lambda_{1}-\lambda_{1}^{0}\right)^{\alpha_{1}}\left(\lambda_{2}-\lambda_{2}^{0}\right)^{\alpha_{2}} \ldots\left(\lambda_{d}-\lambda_{d}^{0}\right)^{\alpha_{d}} .
$$

By Cauchy's estimates we have

$$
\left|a_{\alpha}\right| \leq \frac{M}{R^{|\alpha|}}
$$


for all $\alpha \in \mathbb{N}_{0}^{d}$. We have

$$
\begin{aligned}
& \operatorname{Re} \psi\left(\lambda_{1}, \lambda_{2}, \ldots, \lambda_{d}\right)= \\
& =\sum_{\alpha \in \mathbb{N}_{0}^{d}} \operatorname{Re}\left[a_{\alpha}\left(\sum_{p=0}^{\alpha_{1}}\left(\begin{array}{c}
\alpha_{1} \\
p
\end{array}\right)\left(\operatorname{Re} \lambda_{1}-\operatorname{Re} \lambda_{1}^{0}\right)^{p}\left(\operatorname{Im} \lambda_{1}-\operatorname{Im} \lambda_{1}^{0}\right)^{\alpha_{1}-p} i^{\alpha_{1}-p}\right) .\right. \\
& \text {. }\left(\sum_{p=0}^{\alpha_{2}}\left(\begin{array}{c}
\alpha_{2} \\
p
\end{array}\right)\left(\operatorname{Re} \lambda_{2}-\operatorname{Re} \lambda_{2}^{0}\right)^{p}\left(\operatorname{Im} \lambda_{2}-\operatorname{Im} \lambda_{2}^{0}\right)^{\alpha_{2}-p} i^{\alpha_{2}-p}\right) \cdot \ldots \\
& \left.\ldots \cdot\left(\sum_{p=0}^{\alpha_{d}}\left(\begin{array}{c}
\alpha_{1} \\
p
\end{array}\right)\left(\operatorname{Re} \lambda_{d}-\operatorname{Re} \lambda_{d}^{0}\right)^{p}\left(\operatorname{Im} \lambda_{d}-\operatorname{Im} \lambda_{d}^{0}\right)^{\alpha_{d}-p} i^{\alpha_{d}-p}\right)\right] \\
& =\sum_{\beta \in \mathbb{N}_{0}^{2 d}} \operatorname{Re}\left[a_{\hat{\beta}} \prod_{j=1}^{d}\left(\begin{array}{c}
\beta_{j}^{(1)}+\beta_{j}^{(2)} \\
\beta_{j}^{(1)}
\end{array}\right) i^{\beta_{j}^{(2)}}\left(\operatorname{Re} \lambda_{j}-\operatorname{Re} \lambda_{j}^{0}\right)^{\beta_{j}^{(1)}}\left(\operatorname{Im} \lambda_{j}-\operatorname{Im} \lambda_{j}^{0}\right)^{\beta_{j}^{(2)}}\right] \\
& =\sum_{\beta \in \mathbb{N}_{0}^{2 d}} \operatorname{Re}\left(a_{\hat{\beta}} \prod_{j=1}^{d}\left(\begin{array}{c}
\beta_{j}^{(1)}+\beta_{j}^{(2)} \\
\beta_{j}^{(1)}
\end{array}\right) i^{\beta_{j}^{(2)}}\right)\left(\operatorname{Re} \lambda_{j}-\operatorname{Re} \lambda_{j}^{0}\right)^{\beta_{j}^{(1)}}\left(\operatorname{Im} \lambda_{j}-\operatorname{Im} \lambda_{j}^{0}\right)^{\beta_{j}^{(2)}}
\end{aligned}
$$

where we wrote $\beta \in \mathbb{N}_{0}^{2 d}$ in the form $\left(\beta_{1}^{(1)}, \beta_{1}^{(2)}, \beta_{2}^{(1)}, \beta_{2}^{(2)}, \ldots, \beta_{d}^{(1)}, \beta_{d}^{(2)}\right)$ and we also put $\hat{\beta}=\left(\beta_{1}^{(1)}+\beta_{1}^{(2)}, \beta_{2}^{(1)}+\beta_{2}^{(2)}, \ldots, \beta_{d}^{(1)}+\beta_{d}^{(2)}\right) \in \mathbb{N}_{0}^{d}$. Set

$$
c_{\beta}=\operatorname{Re}\left(a_{\hat{\beta}} \prod_{j=1}^{d}\left(\begin{array}{c}
\beta_{j}^{(1)}+\beta_{j}^{(2)} \\
\beta_{j}^{(1)}
\end{array}\right) i^{\beta_{j}^{(2)}}\right) .
$$

Using (9.1), we get

$$
\left|c_{\beta}\right| \leq\left|a_{\hat{\beta}}\right| \prod_{j=1}^{d}\left(\begin{array}{c}
\beta_{j}^{(1)}+\beta_{j}^{(2)} \\
\beta_{j}^{(1)}
\end{array}\right) \leq M R^{-|\hat{\beta}|} \prod_{j=1}^{d} 2^{\beta_{j}^{(1)}+\beta_{j}^{(2)}}=M R^{-|\beta|} 2^{|\beta|} .
$$

Thus the formula

$$
\Re \psi\left(x_{1}, y_{1}, x_{2}, y_{2}, \ldots, x_{d}, y_{d}\right)=\sum_{\beta \in \mathbb{N}_{0}^{2 d}} c_{\beta} \prod_{j=1}^{d}\left(x_{j}-\operatorname{Re} \lambda_{j}^{0}\right)^{\beta_{j}^{(1)}}\left(y_{j}-\operatorname{Im} \lambda_{j}^{0}\right)^{\beta_{j}^{(2)}}
$$

defines an analytic function on $D_{2 d}\left(\lambda_{0}, R / 4\right)$ and

$$
\left|\Re \psi\left(x_{1}, y_{1}, x_{2}, y_{2}, \ldots, x_{d}, y_{d}\right)\right| \leq 4^{d} M .
$$

Obviously $\left.\Re \psi\right|_{D_{d}\left(\lambda_{0}, R / 4\right)}=\left.\operatorname{Re} \psi\right|_{D_{d}\left(\lambda_{0}, R / 4\right)}$, and we are done.

Lemma 9.2. Suppose that $X$ is a closed subset of $\mathbb{C}$. Fix $R>0$ and $g: B(X, R) \rightarrow$ $\mathbb{R}$, a bounded harmonic function. If $\hat{g}: B(X, R) \rightarrow \mathbb{C}$ is a holomorphic function whose real part is equal to $g$, then

$$
L_{g}^{\prime}:=\sup \left\{\left|\hat{g}^{\prime}(z)\right|: z \in B(X, R / 2)\right\}<\infty .
$$

In particular $\hat{g}: B(X, R / 2) \rightarrow \mathbb{C}$ is Lipschitz continuous with Lipschitz constant $L_{g}^{\prime}$. 
Proof. Since $g: B(X, R) \rightarrow \mathbb{R}$ is bounded, there exists $A \geq 0$ such that $-A \leq g(z) \leq A$ for all $z \in B(X, R)$. Consider the function $G(z)=\exp (\hat{g}(z))$, $z \in B(X, R)$. Then

$$
e^{-A} \leq|G(z)| \leq e^{A}
$$

for all $z \in B(X, R)$. Then for every $z \in B(X, R / 2), B(z, R / 2) \subset B(X, R)$, and it follows from Cauchy's Estimate that

$$
\left|G^{\prime}(z)\right| \leq 2 e^{A} R^{-1} .
$$

Since $G^{\prime}(z)=G(z) \hat{g}^{\prime}(z)$, we get $\hat{g}^{\prime}(z)=G^{\prime}(z) / G(z)$, and applying (9.3) along with (9.2), we obtain that for all $z \in B(X, R / 2)$

$$
\left|\hat{g}^{\prime}(z)\right|=\frac{\left|G^{\prime}(z)\right|}{|G(z)|} \leq 2 e^{2 A} R^{-1} .
$$

We are done.

\subsection{Holomorphic Families and Quasi-Conformal Conjugacies}

Fix $\Lambda$, an open subset of $\mathbb{C}^{d}, d \geq 1$. We say that a family $\mathcal{M}_{\Lambda}=\left\{f_{\lambda}\right\}_{\lambda \in \Lambda}$ of dynamically regular meromorphic maps is analytic if the function $\lambda \mapsto f_{\lambda}(z), \lambda \in \Lambda$, is meromorphic for all $z \in \mathbb{C}$ and the points of the singular set $\operatorname{sing}\left(f_{\lambda}^{-1}\right)$ depend continuously on $\lambda \in \Lambda$. We recall from the introduction that $\mathcal{M}_{\Lambda}$ is of bounded deformation if there is $M>0$ such that for all $j=1, \ldots, N$

$$
\left|\frac{\partial f_{\lambda}(z)}{\partial \lambda_{j}}\right| \leq M\left|f_{\lambda}^{\prime}(z)\right|, \quad \lambda \in \Lambda \text { and } z \in \mathcal{J}\left(f_{\lambda}\right) .
$$

The Speiser class $\mathcal{S}$ is the family of meromorphic functions $f: \mathbb{C} \rightarrow \hat{\mathbb{C}}$ that have a finite set of singular values $\operatorname{sing}\left(f^{-1}\right)$. We will work in the subclass $\mathcal{S}_{0}$ which consists in dynamically regular functions $f \in \mathcal{S}$ that have a strictly positive and finite order $\rho=\rho(f)$ and that are of divergence type.

Recall that the family $\mathcal{M}_{\Lambda} \subset \mathcal{S}_{0}$ is of uniformly balanced growth provided every $f_{\lambda} \in \mathcal{M}_{\Lambda}$ satisfies the condition (2.9) with some fixed constants $\kappa \geq 1, \alpha_{1} \in \mathbb{R}$ and $\underline{\alpha}_{2} \leq \bar{\alpha}_{2}$. We assume further that $\alpha_{1} \geq 0$.

The work of Lyubich and Mañé-Sad-Sullivan [L1, MSS] on the structural stability of rational maps has been generalized to entire functions of the Speiser class by Eremenko-Lyubich $[\mathbf{E L}$. Note also that they show that any entire function of the Speiser class is naturally imbedded in a holomorphic family of functions in which the singular points are local parameters. Here we collect and adapt to the meromorphic setting the facts that are important for our needs. We also give an interpretation of the bounded deformation assumption of $\mathcal{M}_{\Lambda}$ near $f_{\lambda^{0}}$ in terms of a bounded speed of the involved holomorphic motions. A holomorphic motion of a set $A \subset \mathbb{C}$ over $U$ originating at $\lambda^{0}$ is a map $G: U \times A \rightarrow \mathbb{C}$ satisfying the following conditions:

(1) The map $\lambda \mapsto G(\lambda, z)$ is holomorphic for every $z \in A$.

(2) The map $G_{\lambda}: z \mapsto G_{\lambda}(z)=G(\lambda, z)$ is injective for every $\lambda \in U$.

(3) $G_{\lambda^{0}}=i d$.

The $\lambda$-lemma MSS] asserts that such a holomorphic motion extends in a quasiconformal way to the closure of $A$. Further improvements, resulting in the final version of Slodkowski $\left[\mathbf{S k}\right.$, show that each map $G_{\lambda}$ is the restriction of a global 
quasiconformal map of the sphere $\hat{\mathbb{C}}$. We recall that $f_{\lambda_{0}} \in \mathcal{M}_{\Lambda}$ (or simply $\lambda \in \Lambda$ ) is holomorphically J-stable if there is a neighborhood $U \subset \Lambda$ of $\lambda^{0}$ and a holomorphic motion $G_{\lambda}$ of $\mathcal{J}\left(f_{\lambda^{0}}\right)$ over $U$ such that $G_{\lambda}\left(\mathcal{J}\left(f_{\lambda^{0}}\right)\right)=\mathcal{J}\left(f_{\lambda}\right)$ and

$$
G_{\lambda} \circ f_{\lambda^{0}}=f_{\lambda} \circ G_{\lambda} \text { on } \mathcal{J}\left(f_{\lambda^{0}}\right)
$$

for every $\lambda \in U$.

Lemma 9.3. A function $f_{\lambda^{0}} \in \mathcal{M}_{\Lambda}$ is holomorphically J-stable if and only if for every singular value $a_{j, \lambda^{0}} \in \operatorname{sing}\left(f_{\lambda^{0}}^{-1}\right)$ the family of functions

$$
\lambda \mapsto f_{\lambda}^{n}\left(a_{j, \lambda}\right), \quad n \geq 1,
$$

is normal in a neighborhood of $\lambda^{0}$.

PROOF. This can be proved precisely like for rational functions because the functions in the Speiser class $\mathcal{S}$ do not have wandering nor Baker domains (see L2] or BM, p. 102]).

From this criterion together with the description of the components of the Fatou set one easily deduces the following.

Lemma 9.4. If $\mathcal{M}_{\Lambda}$ is an analytic family of bounded deformation and uniformly balanced growth, then each element $f_{\lambda^{0}} \in \mathcal{M}_{\Lambda}$ is holomorphically $J$-stable.

We now investigate the speed of the associated holomorphic motion.

Proposition 9.5. Suppose that $\mathcal{M}_{\Lambda}$ is an analytic family of bounded deformation and uniformly balanced growth. Fix $\lambda^{0} \in \Lambda$ and let $G_{\lambda}$ be the associated holomorphic motion over $\Lambda$ (cf. Lemma 9.4). Then there is a constant $C>0$ such that

$$
\left|\frac{\partial G_{\lambda}(z)}{\partial \lambda_{j}}\right| \leq C
$$

for every $\lambda \in U$, a sufficiently small neighbourhood of $\lambda^{0} \in \Lambda$, and every $z \in \mathcal{J}\left(f_{\lambda^{0}}\right)$ and $j=1, \ldots, d$. It follows that $G_{\lambda}$ converges to the identity map uniformly on $\mathcal{J}\left(f_{\lambda^{0}}\right) \cap \mathbb{C}$ (in the Euclidean metric) and, replacing $U$ by a smaller neighborhood if necessary, there exists $0<\tau \leq 1$ such that $G_{\lambda}$ is $\tau$-Hölder for every $\lambda \in U$.

Proof. Let $G_{\lambda}$ be the holomorphic motion such that $f_{\lambda} \circ G_{\lambda}=G_{\lambda} \circ f_{\lambda^{0}}$ on $\mathcal{J}\left(f_{\lambda^{0}}\right)$ for $\lambda \in U$ and such that there are $c>0$ and $\gamma>1$ for which

$$
\left|\left(f_{\lambda}^{n}\right)^{\prime}(z)\right| \geq c \gamma^{n} \quad \text { for every } n \geq 1, z \in \mathcal{J}_{f_{\lambda}} \text { and } \lambda \in U .
$$

(cf. Fact 4.5). Denote $z_{\lambda}=G_{\lambda}(z)$ and consider

$$
F_{n}(\lambda, z)=f_{\lambda}^{n}\left(z_{\lambda}\right)-z_{\lambda} .
$$

The derivative of this function with respect to $\lambda_{j}$ gives

$$
\frac{\partial}{\partial \lambda_{j}} F_{n}(\lambda, z)=\frac{\partial f_{\lambda}^{n}}{\partial \lambda_{j}}\left(G_{\lambda}(z)\right)+\left(f_{\lambda}^{n}\right)^{\prime}\left(G_{\lambda}(z)\right) \frac{\partial}{\partial \lambda_{j}} G_{\lambda}(z)-\frac{\partial}{\partial \lambda_{j}} G_{\lambda}(z) .
$$


Suppose that $z$ is a repelling periodic point of period $n$. Then $\lambda \mapsto F_{n}(\lambda, z) \equiv 0$ and it follows from (9.5) that

$$
\left|\frac{\partial G_{\lambda}(z)}{\partial \lambda_{j}}\right|=\left|\frac{\frac{\partial f_{\lambda}^{n}}{\partial \lambda_{j}}\left(z_{\lambda}\right)}{1-\left(f_{\lambda}^{n}\right)^{\prime}\left(z_{\lambda}\right)}\right| \preceq\left|\frac{\frac{\partial f_{\lambda}^{n}}{\partial \lambda_{j}}\left(z_{\lambda}\right)}{\left(f_{\lambda}^{n}\right)^{\prime}\left(z_{\lambda}\right)}\right|=\Delta_{n, j} .
$$

Since $\frac{\partial f_{\lambda}^{n}}{\partial \lambda_{j}}\left(z_{\lambda}\right)=\frac{\partial f_{\lambda}}{\partial \lambda_{j}}\left(f_{\lambda}^{n-1}\left(z_{\lambda}\right)\right)+f_{\lambda}^{\prime}\left(f_{\lambda}^{n-1}\left(z_{\lambda}\right)\right) \frac{\partial f_{\lambda}^{n-1}}{\partial \lambda_{j}}\left(z_{\lambda}\right)$ we have

$$
\Delta_{n, j} \leq \frac{\left|\frac{\partial f_{\lambda}}{\partial \lambda_{j}}\left(f_{\lambda}^{n-1}\left(z_{\lambda}\right)\right)\right|}{\left|f_{\lambda}^{\prime}\left(f_{\lambda}^{n-1}\left(z_{\lambda}\right)\right)\right|} \frac{1}{\left|\left(f_{\lambda}^{n-1}\right)^{\prime}\left(z_{\lambda}\right)\right|}+\Delta_{n-1, j} .
$$

Making use of the expanding (9.5) and the bounded deformation (9.4) properties it follows that

$$
\Delta_{n, j} \leq \frac{M}{c \gamma^{n-1}}+\Delta_{n-1, j}
$$

The conclusion comes now from the density of the repelling cycles in the Julia set $\mathcal{J}\left(f_{\lambda^{0}}\right)$ :

$$
\left|\frac{\partial G_{\lambda}(z)}{\partial \lambda_{j}}\right| \preceq \frac{M}{c} \frac{\gamma}{\gamma-1} \text { for every } z \in \mathcal{J}\left(f_{\lambda^{0}}\right) .
$$

The Hölder continuity property is now standard (see [UZ2]).

\subsection{Real Analyticity of the Multifractal Function}

Keep notation and terminology from the previous section. Fix $t>\rho / \hat{\tau}, \lambda_{0} \in \Lambda$, and a bounded harmonic function $h: B\left(\mathcal{J}\left(f_{\lambda^{0}}\right), R\right) \rightarrow \mathbb{R}$ with some $R \in(0, \delta)$. By $J$ stability of $f_{\lambda_{0}}$ proven in Lemma 9.4 there exists a sufficiently small neighbourhood $U \subset \Lambda$ of $\lambda^{0}$ such that $J\left(f_{\lambda}\right) \subset B\left(\mathcal{J}\left(f_{\lambda^{0}}\right), R / 2\right)$ for all $\lambda \in U$. Then for all $\lambda \in U$ the function

$$
\phi_{\lambda}=-t \log \left|f_{\lambda}^{\prime}\right|_{\tau}+h: B\left(\mathcal{J}\left(f_{\lambda^{0}}\right), R\right) \rightarrow \mathbb{R}
$$

restricted to $J\left(f_{\lambda}\right)$ is a tame function with respect to the map $f_{\lambda}: \mathbb{C} \rightarrow \hat{\mathbb{C}}$. We prove first the following.

Lemma 9.6. Both functions $z \mapsto h \circ G_{\lambda}(z)-h(z)$ and $z \mapsto \log \left|f_{\lambda}^{\prime}\left(G_{\lambda}(z)\right)\right|_{\tau}-$ $\log \left|f_{\lambda^{0}}^{\prime}(z)\right|_{\tau}, z \in \mathcal{J}\left(f_{\lambda^{0}}\right)$, are weakly $\beta$-Hölder on a sufficiently small neighbourhood $U$ of $\lambda^{0} \in \Lambda$. The corresponding $\beta$-variations are uniformly bounded above, say by $V$.

Proof. By Lemma 9.2 and by Proposition 9.5 the function $z \mapsto h \circ G_{\lambda}(z)-h(z)$ is $\beta$-Hölder continuous $(\beta<1)$ with the $\beta$-variation uniformly bounded above in a sufficiently small neighbourhood of $\lambda^{0}$. By Corollary 4.8 the function $\log \left|f_{\lambda^{0}}^{\prime}(z)\right|_{\tau}$ is weakly Lipshitz. Using again Corollary 4.8 along with Proposition 9.5, we get 
for all $v \in \mathcal{J}(f)$ and all $z, w \in D(f(v), \delta)$ that

$$
\begin{aligned}
|| \log \left|f_{\lambda}^{\prime}\left(G_{\lambda}\left(f_{\lambda^{0}, v}^{-1}(w)\right)\right)\right|_{\tau}-\log \left|f_{\lambda}^{\prime}\left(G_{\lambda}\left(f_{\lambda^{0}, v}^{-1}(z)\right)\right)\right|_{\tau} \mid= \\
\quad=\left.|\log | f_{\lambda}^{\prime}\left(f_{\lambda, G_{\lambda}(v)}^{-1}\left(G_{\lambda}(w)\right)\right)\right|_{\tau}-\log \left|f_{\lambda}^{\prime}\left(f_{\lambda, G_{\lambda}(v)}^{-1}\left(G_{\lambda}(z)\right)\right)\right|_{\tau} \mid \\
\quad=\left.|\log |\left(f_{\lambda, G_{\lambda}(v)}^{-1}\right)^{\prime}\left(G_{\lambda}(z)\right)\right|_{\tau}-\log \left|\left(f_{\lambda, G_{\lambda}(v)}^{-1}\right)^{\prime}\left(G_{\lambda}(w)\right)\right|_{\tau} \mid \\
\preceq\left|G_{\lambda}(z)-G_{\lambda}(w)\right| \preceq|w-z|^{\beta} .
\end{aligned}
$$

We are done.

Denote $z_{\lambda}=G_{\lambda}(z), z \in \mathcal{J}\left(f_{\lambda^{0}}\right)$ and $\lambda \in \mathbb{D}_{\mathbb{C}^{d}}\left(\lambda^{0}, R\right)$. Remember that $G_{\lambda} \rightarrow i d$ uniformly in $\mathcal{J}\left(f_{\lambda^{0}}\right)$ (Proposition 9.5). Since $0 \notin \mathcal{J}\left(f_{\lambda^{0}}\right)$ the function

$$
\Psi_{z}(\lambda)=\frac{f_{\lambda}^{\prime}\left(z_{\lambda}\right)}{f_{\lambda^{0}}^{\prime}(z)}\left(\frac{z_{\lambda}}{z}\right)^{\tau}\left(\frac{f_{\lambda^{0}}(z)}{f_{\lambda}\left(z_{\lambda}\right)}\right)^{\tau}
$$

is well defined on the simply connected domain $\mathbb{D}_{\mathbb{C}^{d}}\left(\lambda^{0}, R\right)$. Here we choose $w \mapsto w^{\tau}$ so that this map fixes 1 which implies that

$$
\Psi_{z}\left(\lambda^{0}\right)=1 \quad \text { for every } z \in \mathcal{J}_{0}=\mathcal{J}\left(f_{\lambda^{0}}\right) \backslash f_{\lambda^{0}}^{-1}(\infty) .
$$

For this function one has the following uniform estimate.

Lemma 9.7. For every $\varepsilon>0$ there is $0<r_{\varepsilon}<R$ such that $\left|\Psi_{z}(\lambda)-1\right|<\varepsilon$ for every $\lambda \in \mathbb{D}_{\mathbb{C}^{d}}\left(\lambda^{0}, r_{\varepsilon}\right)$ and every $z \in \mathcal{J}_{0}$.

Proof. Suppose to the contrary that there is $\varepsilon>0$ such that for some $r_{j} \rightarrow 0$ there exists $\lambda_{j} \in \mathbb{D}_{\mathbb{C}^{d}}\left(\lambda^{0}, r_{j}\right)$ and $z_{j} \in \mathcal{J}_{0}$ with $\left|\Psi_{z_{j}}\left(\lambda_{j}\right)-1\right|>\varepsilon$. Then the family of functions

$$
\mathcal{F}=\left\{\Psi_{z} ; z \in \mathcal{J}_{0}\right\}
$$

cannot be normal on any domain $\mathbb{D}_{\mathbb{C}^{d}}\left(\lambda^{0}, r\right), 0<r<R$. This is however not true. Indeed, the uniform balanced growth condition (Definition 2.9) yields

$$
\left|\Psi_{z}(\lambda)\right| \leq \kappa^{2} \frac{\left|z_{\lambda}\right|^{\alpha_{1}}\left|f_{\lambda}\left(z_{\lambda}\right)\right|^{\alpha_{2, \lambda}\left(z_{\lambda}\right)}}{|z|^{\alpha_{1}}\left|f_{\lambda^{0}}(z)\right|^{\alpha_{2}(z)}}\left|\frac{z_{\lambda}}{z}\right|^{\tau}\left|\frac{f_{\lambda^{0}}(z)}{f_{\lambda}\left(z_{\lambda}\right)}\right|^{\tau}=\kappa^{2}\left|\frac{z_{\lambda}}{z}\right|^{\hat{\tau}}\left|\frac{f_{\lambda}\left(z_{\lambda}\right)}{f_{\lambda^{0}}(z)}\right|^{\alpha_{2}(z)-\tau}
$$

for every $z \in \mathcal{J}_{0}$ and $\left|\lambda-\lambda^{0}\right|<R$. Since $f_{\lambda}\left(z_{\lambda}\right)=G_{\lambda} \circ f_{\lambda^{0}}(z), G_{\lambda} \rightarrow I d$ uniformly in $\mathbb{C}$ and since $\alpha_{2}(z) \leq \bar{\alpha}$ it follows immediately that $\mathcal{F}$ is normal on some disk $\mathbb{D}_{\mathbb{C}^{d}}\left(\lambda^{0}, r\right), 0<r<R$.

Let $\log : D(1,1) \rightarrow \mathbb{C}$ be the branch of logarithm uniquely determined by the requirement that $\log 1=0$. In view of Lemma 9.7 for every $z \in \mathcal{J}\left(f_{\lambda^{0}}\right)$, the function $\lambda \mapsto \log \Psi_{z}, \lambda \in D\left(\lambda_{0}, r\right) \subset U$, is analytic and bounded above by $\log 2$. Consider its Taylor series expansion

$$
\log \Psi_{z}(\lambda)=\sum_{\alpha \in \mathbb{N}_{0}^{d}} a_{\alpha}(z)\left(\lambda_{1}-\lambda_{1}^{0}\right)^{\alpha_{1}}\left(\lambda_{2}-\lambda_{2}^{0}\right)^{\alpha_{2}} \ldots\left(\lambda_{d}-\lambda_{d}^{0}\right)^{\alpha_{d}} .
$$

By the definition of holomorphic motion, Lemma 9.2, and Lemma 9.4, for every $z \in \mathcal{J}\left(f_{\lambda^{0}}\right)$, the function $\left.\lambda \mapsto \hat{(} h\left(G_{\lambda}(z)\right)-h(z)\right), \lambda \in D\left(\lambda_{0}, r\right)$, is analytic and 
bounded above by $2\|h\|_{\infty}$. Consider its Taylor series expansion

$$
\Delta_{z}(\lambda)=\sum_{\alpha \in \mathbb{N}_{0}^{d}} b_{\alpha}(z)\left(\lambda_{1}-\lambda_{1}^{0}\right)^{\alpha_{1}}\left(\lambda_{2}-\lambda_{2}^{0}\right)^{\alpha_{2}} \ldots\left(\lambda_{d}-\lambda_{d}^{0}\right)^{\alpha_{d}} .
$$

It follows from the proof of Lemma 9.1, that with its notation, the series

$$
\Re \log \Psi_{z}\left(x_{1}, y_{1}, x_{2}, y_{2}, \ldots, x_{d}, y_{d}\right)=\sum_{\gamma \in \mathbb{N}_{0}^{2 d}} A_{\gamma}(z) \prod_{j=1}^{d}\left(x_{j}-\operatorname{Re} \lambda_{j}^{0}\right)^{\gamma_{j}^{(1)}}\left(y_{j}-\operatorname{Im} \lambda_{j}^{0}\right)^{\gamma_{j}^{(2)}}
$$

and

$$
\Re \Delta_{z}\left(x_{1}, y_{1}, x_{2}, y_{2}, \ldots, x_{d}, y_{d}\right)=\sum_{\gamma \in \mathbb{N}_{0}^{2 d}} B_{\gamma}(z) \prod_{j=1}^{d}\left(x_{j}-\operatorname{Re} \lambda_{j}^{0}\right)^{\gamma_{j}^{(1)}}\left(y_{j}-\operatorname{Im} \lambda_{j}^{0}\right)^{\gamma_{j}^{(2)}}
$$

define analytic functions on $D_{2 d}\left(\lambda_{0}, r / 4\right)$ and $\left.\Re \log \Psi_{z}\right|_{D_{d}\left(\lambda_{0}, r / 4\right)}=\operatorname{Re} \log \Psi_{z}=$ $\log \left|\Psi_{z}\right|$ and $\left.\left.\Re \Delta_{z}\right|_{D_{d}\left(\lambda_{0}, r / 4\right)}=\operatorname{Re} \Delta_{z}=h\left(G_{(\cdot)}(z)\right)-h(z)\right)$. In here

$$
A_{\gamma}(z)=\operatorname{Re}\left(a_{\hat{\gamma}}(z) \prod_{j=1}^{d}\left(\begin{array}{c}
\gamma_{j}^{(1)}+\gamma_{j}^{(2)} \\
\gamma_{j}^{(1)}
\end{array}\right) i^{\gamma_{j}^{(2)}}\right) .
$$

and

$$
B_{\gamma}(z)=\operatorname{Re}\left(b_{\hat{\gamma}}(z) \prod_{j=1}^{d}\left(\begin{array}{c}
\gamma_{j}^{(1)}+\gamma_{j}^{(2)} \\
\gamma_{j}^{(1)}
\end{array}\right) i^{\gamma_{j}^{(2)}}\right) .
$$

By Lemma 9.6, it follows from Cauchy's estimates that for all $\alpha \in \mathbb{N}_{0}^{d}$, all $v \in \mathcal{J}\left(f_{\lambda^{0}}\right)$ and all $z, w \in D(f(v), \delta)$, we have

$$
\left|a_{\alpha}\left(f_{\lambda^{0}, v}^{-1}(w)\right)-a_{\alpha}\left(f_{\lambda^{0}, v}^{-1}(z)\right)\right|,\left|b_{\alpha}\left(f_{\lambda^{0}, v}^{-1}(w)\right)-b_{\alpha}\left(f_{\lambda^{0}, v}^{-1}(z)\right)\right| \leq V r^{-|\alpha|}|w-z|^{\beta}
$$

Therefore, for every $\gamma \in \mathbb{N}_{0}^{2 d}$, we get

$$
\begin{aligned}
& \left|A_{\gamma}\left(f_{\lambda^{0}, v}^{-1}(w)\right)-A_{\gamma}\left(f_{\lambda^{0}, v}^{-1}(z)\right)\right|= \\
& \quad=\left|\operatorname{Re}\left(a_{\hat{\gamma}}\left(f_{\lambda^{0}, v}^{-1}(w)\right) i^{\gamma_{j}^{(2)}}\right)-\operatorname{Re}\left(a_{\hat{\gamma}}\left(f_{\lambda^{0}, v}^{-1}(z)\right) i^{\gamma_{j}^{(2)}}\right)\right| \prod_{j=1}^{d}\left(\begin{array}{c}
\gamma_{j}^{(1)}+\gamma_{j}^{(2)} \\
\gamma_{j}^{(1)}
\end{array}\right) i^{\gamma_{j}^{(2)}} \\
& \quad \leq\left|a_{\hat{\gamma}}\left(f_{\lambda^{0}, v}^{-1}(w)\right) i^{\gamma_{j}^{(2)}}-a_{\hat{\gamma}}\left(f_{\lambda^{0}, v}^{-1}(z)\right) i^{\gamma_{j}^{(2)}}\right| \prod_{j=1}^{d}\left(\begin{array}{c}
\gamma_{j}^{(1)}+\gamma_{j}^{(2)} \\
\gamma_{j}^{(1)}
\end{array}\right) i^{\gamma_{j}^{(2)}} \\
& \quad=\mid a_{\hat{\gamma}}\left(f_{\lambda^{0}, v}^{-1}(w)\right)-\left(a_{\hat{\gamma}}\left(f_{\lambda^{0}, v}^{-1}(z)\right) \mid \prod_{j=1}^{d}\left(\begin{array}{c}
\gamma_{j}^{(1)}+\gamma_{j}^{(2)} \\
\gamma_{j}^{(1)}
\end{array}\right) i^{\gamma_{j}^{(2)}}\right. \\
& \quad \leq 2^{|\gamma|} \mid a_{\hat{\gamma}}\left(f_{\lambda^{0}, v}^{-1}(w)\right)-\left(a_{\hat{\gamma}}\left(f_{\lambda^{0}, v}^{-1}(z)\right) \mid\right. \\
& \quad \leq 2^{|\gamma|} V r^{-|\gamma|}|w-z|^{\beta} .
\end{aligned}
$$

and similarly,

$$
\left|B_{\gamma}\left(f_{\lambda^{0}, v}^{-1}(w)\right)-B_{\gamma}\left(f_{\lambda^{0}, v}^{-1}(z)\right)\right| \leq 2^{|\gamma|} V r^{-|\gamma|}|w-z|^{\beta} .
$$

Now we can prove the following. 
Lemma 9.8. Fix $\left(q_{0}, T_{0}\right) \in \mathbb{R}^{2}$ such that $q_{0} t+T_{0}>\rho / \hat{\tau}$. Then, with $r>0$ as above, so small that $\left(q_{0}-(r / 4)\right) t+T_{0}-(r / 4)>\rho / \hat{\tau}$, for every $(\lambda, q, T) \in$ $D_{4}\left(\left(\lambda_{0}, q_{0}, T_{0}\right), r / 4\right)$, the function

$$
\zeta_{\lambda, q, T}:=-(q t+T) \Re \log \Psi_{(\cdot)}(\lambda)+q \Re \Delta_{(\cdot)}(\lambda): \mathcal{J}\left(f_{\lambda^{0}}\right) \rightarrow \mathbb{C}
$$

is a member of $\mathrm{H}_{\beta}^{w}$ and

$$
\sup \left\{\left.||\left|\zeta_{\lambda, q, T}\right|\right|_{\beta}:(\lambda, q, T) \in D_{4}\left(\left(\lambda_{0}, q_{0}, T_{0}\right), r / 4\right)\right\}<\infty .
$$

Proof. It follows from Lemma 9.1 that for all $(\lambda, q, T) \in D_{4}\left(\left(\lambda_{0}, q_{0}, T_{0}\right), r / 4\right)$, (9.8) $\left\|\zeta_{\lambda, q, T}\right\|_{\infty} \leq 4^{4}\left(2\left(\left|q_{0}\right|+(r / 4)\right)\|h\|_{\infty}+\log 2\left(\left(\left|q_{0}\right|+(r / 4)\right)|t|+\left|T_{0}\right|+(r / 4)\right)\right)$.

Put $Q_{1}=\left|q_{0}\right|+(r / 4)$ and $Q_{2}=\left(\left|q_{0}\right|+(r / 4)\right)|t|+\left|T_{0}\right|+(r / 4)$. It follows from (9.6) and (9.6) that for all $(\lambda, q, T) \in D_{4}\left(\left(\lambda_{0}, q_{0}, T_{0}\right), r / 8\right)$, all $v \in \mathcal{J}\left(f_{\lambda^{0}}\right)$ and all $z, w \in D(f(v), \delta)$, writing $\lambda=\left(\lambda_{1,1}, \lambda_{1,2}, \lambda_{2,1}, \lambda_{2,2} \ldots, \lambda_{d, 1}, \lambda_{d, 2}\right)$, we have

$$
\begin{aligned}
& \left|\zeta_{\lambda, q, T}\left(f_{\lambda^{0}, v}^{-1}(w)\right)-\zeta_{\lambda, q, T}\left(f_{\lambda^{0}, v}^{-1}(z)\right)\right|= \\
& =\mid-(q t+T)\left(\Re \log \Psi_{\left(f_{\lambda^{0}, v}^{-1}(w)\right)}(\lambda)-\Re \log \Psi_{\left(f_{\lambda^{0}, v}^{-1}(z)\right)}(\lambda)\right)+ \\
& \quad+q\left(\Re \Delta_{\left(f_{\lambda^{0}, v}^{-1}(w)\right)}(\lambda)-\Re \Delta_{\left(f_{\lambda^{0}, v}^{-1}(z)\right)}(\lambda)\right) \mid \\
& \leq Q_{2}\left|\Re \log \Psi_{\left(f_{\lambda^{0}, v}^{-1}(w)\right)}(\lambda)-\Re \log \Psi_{\left(f_{\lambda^{0}, v}^{-1}(z)\right)}(\lambda)\right| \\
& \quad+Q_{1}\left|\Re \Delta_{\left(f_{\lambda^{0}, v}^{-1}(w)\right)}(\lambda)-\Re \Delta_{\left(f_{\lambda^{0}, v}^{-1}(z)\right)}(\lambda)\right| \\
& \leq Q_{2} \sum_{\gamma \in \mathbb{N}_{0}^{2 d}} \prod_{j=1}^{d}\left(\lambda_{j, 1}-\operatorname{Re} \lambda_{j}^{0}\right)^{\gamma_{j}^{(1)}}\left(\lambda_{j, 2}-\operatorname{Im} \lambda_{j}^{0}\right)^{\gamma_{j}^{(2)}}\left|B_{\gamma}\left(f_{\lambda^{0}, v}^{-1}(w)\right)-B_{\gamma}\left(f_{\lambda^{0}, v}^{-1}(z)\right)\right|+ \\
& \quad+Q_{1} \sum_{\gamma \in \mathbb{N}_{0}^{2 d}} \prod_{j=1}^{d}\left(\lambda_{j, 1}-\operatorname{Re} \lambda_{j}^{0}\right)^{\gamma_{j}^{(1)}}\left(\lambda_{j, 2}-\operatorname{Im} \lambda_{j}^{0}\right)^{\gamma_{j}^{(2)}}\left|A_{\gamma}\left(f_{\lambda^{0}, v}^{-1}(w)\right)-A_{\gamma}\left(f_{\lambda^{0}, v}^{-1}(z)\right)\right| \\
& \leq\left(Q_{1}+Q_{2}\right) \sum_{\gamma \in \mathbb{N}_{0}^{2 d}} V r^{-|\gamma|} 2^{|\gamma|}|w-z|^{\beta}(r / 4)^{|\gamma|} \\
& =\left(Q_{1}+Q_{2}\right) V|w-z|^{\beta} \sum_{\gamma \in \mathbb{N}_{0}^{2 d}} 2^{-|\gamma|} \\
& =4^{d} V\left(Q_{1}+Q_{2}\right)|w-z|^{\beta} .
\end{aligned}
$$

So, $V_{\beta}\left(\zeta_{\lambda, q, T}\right) \leq 4^{d} V\left(Q_{1}+Q_{2}\right)$ and we are done.

Now we obtain easily the following key technical result of this section.

Lemma 9.9. For every $(\lambda, q, T) \in D_{4}\left(\left(\lambda_{0}, q_{0}, T_{0}\right), r / 4\right)$, the function

$$
\phi_{\lambda, q, T}=-(q t+T) \log \left|f_{\lambda_{0}}^{\prime}\right|_{\tau}+q h+\zeta_{\lambda, q, T}: \mathcal{J}\left(f_{\lambda^{0}}\right) \rightarrow \mathbb{C}
$$

is a $\beta$-tame potential, the map $(\lambda, q, T) \rightarrow \mathcal{L}_{\phi_{\lambda, q, T}} \in L\left(\mathrm{H}_{\beta}\left(\mathcal{J}\left(f_{\lambda^{0}}\right)\right)\right),(\lambda, q, T) \in$ $D_{4}\left(\left(\lambda_{0}, q_{0}, T_{0}\right), r / 4\right)$, is holomorphic and

$$
\phi_{\lambda, q, T}=\left(q \phi_{\lambda}-T \log \left|f_{\lambda}^{\prime}\right|_{\tau}\right) \circ G_{\lambda},
$$

for all $(\lambda, q, T) \in D\left(\lambda_{0}, r / 4\right) \times\left(q_{0}-(r / 4), q_{0}+(r / 4)\right) \times\left(T_{0}-(r / 4), T_{0}+(r / 4)\right)$. 
Proof. Lemma 9.8 implies that $\phi_{\lambda, q, T}: \mathcal{J}\left(f_{\lambda^{0}}\right) \rightarrow \mathbb{C}$ is a weakly $\beta$-tame potential. The choice of $q_{0}, T_{0}$ and $r>0$ (see Lemma 9.8 assures that this potential is tame and that condition (d) of Theorem 7.2 is satisfied. Thus the condition (a) of Theorem 7.2 is satisfied. Since the function $(q, T) \mapsto q t+T$ is holomorphic and since for every $z \in \mathcal{J}\left(f_{\lambda^{0}}\right)$, the function $(\lambda, q, T) \mapsto \zeta_{\lambda, q, T}(z)$ is holomorphic (as the functions $\Re \log \Psi_{z}$ and $\Re \Delta_{z}$ are), the conditions (b) and (c) of Theorem 7.2 are satisfied. Thus Theorem 7.2 applies (with $G=D_{4}\left(\left(\lambda_{0}, q_{0}, T_{0}\right), r / 4\right)$ ) and yields analyticity of the map $(\lambda, q, T) \rightarrow \mathcal{L}_{\phi_{\lambda, q, T}} \in L\left(\mathrm{H}_{\beta}\left(\mathcal{J}\left(f_{\lambda^{0}}\right)\right)\right),(\lambda, q, T) \in D_{4}\left(\left(\lambda_{0}, q_{0}, T_{0}\right), r / 4\right)$. The last assertion of this lemma is obtained by the following calculation. Fix $(\lambda, q, T) \in D\left(\lambda_{0}, r / 4\right) \times\left(q_{0}-(r / 4), q_{0}+(r / 4)\right) \times\left(T_{0}-(r / 4), T_{0}+(r / 4)\right)$. Then, for all $z \in \mathcal{J}\left(f_{\lambda^{0}}\right)$, we get

$$
\begin{aligned}
\phi_{\lambda, q, T}= & -(q t+T) \log \left|f_{\lambda_{0}}^{\prime}(z)\right|_{\tau}+q h(z)-(q t+T) \Re \log \Psi_{z}(\lambda)+q \Re \Delta_{z}(\lambda) \\
= & -(q t+T) \log \left|f_{\lambda_{0}}^{\prime}(z)\right|_{\tau}+q h(z)-(q t+T) \log \left|\Psi_{z}(\lambda)\right| \\
& \quad+q\left(h \circ G_{\lambda}(z)-h(z)\right) \\
= & -(q t+T) \log \left|f_{\lambda_{0}}^{\prime}(z)\right|_{\tau}-(q t+T)\left(\log \left|f_{\lambda}^{\prime} \circ G_{\lambda}(z)\right|_{\tau}\right. \\
& \quad-\log \left|f_{\lambda_{0}}^{\prime}(z)\right|+q h \circ G_{\lambda}(z) \\
= & \left(-(q t+T)\left(\log \left|f_{\lambda}^{\prime}\right|_{\tau}+q h\right) \circ G_{\lambda}(z)\right. \\
= & \left(q \phi_{\lambda}-T \log \left|f_{\lambda}^{\prime}\right|_{\tau}\right) \circ G_{\lambda}(z) .
\end{aligned}
$$

We are done.

For every $(\lambda, q, T) \in \Lambda \times \Sigma_{2}\left(\phi_{\lambda},-\log \left|f_{\lambda}^{\prime}\right|_{\tau}\right)$, let

$$
\mathrm{P}_{\lambda}(q, T)=\mathrm{P}\left(q \phi_{\lambda}-T \log \left|f_{\lambda}^{\prime}\right|_{\tau}\right)
$$

obviously taken with respect to the dynamical system $f_{\lambda}: \mathbb{C} \rightarrow \hat{\mathbb{C}}$. Fix now $\lambda_{0} \in \Lambda$ and $\left(q_{0}, T_{0}\right) \in \mathbb{R}^{2}$ such that $q_{0} t+T_{0}>\rho / \hat{\tau}$, i.e. $\left(q_{0}, T_{0}\right) \in \Sigma_{2}\left(\phi_{\lambda},-\log \left|f_{\lambda}^{\prime}\right|_{\tau}\right) \cap \mathbb{R}^{2}$ assuming that $\lambda \in D\left(\lambda_{0}, r / 4\right)$ with $r / 4$ sufficiently small as above. Since the maps $f_{\lambda}$ and $f_{\lambda_{0}}$ are topologically conjugate on their respective Julia sets via the map $G_{\lambda}$, we get, using Lemma 9.9 that

$$
\mathrm{P}_{\lambda}(q, T)=\mathrm{P}\left(\phi_{\lambda, q, T}\right)
$$

where the topological pressure on the right-hand side of this equality is taken with respect to the dynamical system $f_{\lambda_{0}}: \mathbb{C} \rightarrow \hat{\mathbb{C}}$. Now we can prove the following.

Corollary 9.10. The function $(\lambda, q, t) \mapsto \mathrm{P}_{\lambda}(q, T),(\lambda, q, T) \in \Lambda \times \Sigma_{2}(\phi, \psi) \cap \mathbb{R}^{2}$, is real-analytic.

Proof. Keep $\lambda_{0} \in \Lambda$ and $\left(q_{0}, T_{0}\right) \in \Sigma_{2}\left(\phi_{\lambda},-\log \left|f_{\lambda}^{\prime}\right|_{\tau}\right) \cap \mathbb{R}^{2}$ fixed. Since, by Lemma 9.9, $\phi_{\lambda, q, T}: \mathcal{J}\left(f_{\lambda^{0}}\right) \rightarrow \mathbb{R}$ is a $\beta$-tame potential, using (9.10), it follows from Theorem 6.5 that $\exp \left(\mathrm{P}_{\lambda}(q, T)\right)\left((\lambda, q, T) \in D\left(\lambda_{0}, r / 4\right) \times\left(q_{0}-(r / 4), q_{0}+\right.\right.$ $\left.(r / 4)) \times\left(T_{0}-(r / 4), T_{0}+(r / 4)\right)\right)$ is a simple isolated eigenvalue of the operator $\mathcal{L}_{\phi_{\lambda, q, T}} \in L\left(\mathrm{H}_{\beta}\left(\mathcal{J}\left(f_{\lambda^{0}}\right)\right)\right)$. Hence, in view of analyticity part of Lemma 9.9. KatoRellich Perturbation Theorem ( $\mathbf{R e S i}$, Theorem XII.8 cf. [ $\mathbf{K a}]$ ) is applicable to yield $r_{1} \in(0, r / 4]$ and a holomorphic function $\gamma: D_{4}\left(\left(\lambda_{0}, q_{0}, T_{0}\right), r_{1}\right) \rightarrow \mathbb{C}$ such that $\gamma\left(\lambda_{0}, q_{0}, t_{0}\right)=\exp \left(\mathrm{P} \lambda_{0}\left(q_{0}, T_{0}\right)\right)$ and $g(\lambda, q, t)$ is a simple isolated eigenvalue of the operator $\mathcal{L}_{\phi_{\lambda, q, T}} \in L\left(\mathrm{H}_{\beta}\left(\mathcal{J}\left(f_{\lambda^{0}}\right)\right)\right)$ for every $(\lambda, q, T) \in D_{4}\left(\left(\lambda_{0}, q_{0}, T_{0}\right), r_{1}\right)$ with the 
remainder of the spectrum uniformly separated from $\gamma(\lambda, t)$. In particular there exists $r_{2} \in\left(0, r_{1}\right]$ and $\eta>0$ such that

$$
\sigma\left(\mathcal{L}_{\phi_{\lambda, q, T}}\right) \cap D\left(\exp \left(\mathrm{P} \lambda_{0}\left(q_{0}, T_{0}\right)\right), \eta\right)=\{\gamma(\lambda, t)\}
$$

for all $(\lambda, q, T) \in D_{4}\left(\left(\lambda_{0}, q_{0}, T_{0}\right), r_{2}\right)$. Since $\exp \left(\mathrm{P} \lambda_{0}\left(q_{0}, T_{0}\right)\right)$ is equal to the spectral radius $r\left(\mathcal{L}_{\phi_{\lambda_{0}, q_{0}, T_{0}}}\right)$ of the operator $\mathcal{L}_{\phi_{\lambda_{0}, q_{0}, T_{0}}}$, in view of semi-continuity of the spectral set function (see Theorem 10.20 on p.256 in [Ru] ), taking $r_{2}$ appropriately smaller, we also have that $r\left(\mathcal{L}_{\phi_{\lambda, q, T}}\right) \in\left[0, \exp \left(\mathrm{P} \lambda_{0}\left(q_{0}, T_{0}\right)\right)+\eta\right)$. Along with (9.11), this implies that $\exp \left(\mathrm{P}_{\lambda}(q, T)\right)=\gamma(\lambda, t)$. Consequently, the function $(\lambda, t) \mapsto$ $\mathrm{P}_{\lambda}(q, T),(\lambda, q, T) \in D_{4}\left(\left(\lambda_{0}, q_{0}, T_{0}\right), r_{2}\right)$ is real-analytic.

Our first geometric result, proved in $\mathbf{M y U 2}$ concerns real analyticity of the Hausdorff dimension of the radial Julia sets $J_{r}\left(f_{\lambda}\right)$, which is based on the corollary above and on Theorem 8.3 (Bowen's formula).

Theorem 9.11. If $\mathcal{M}_{\Lambda}$ is an analytic family of bounded deformation and uniformly balanced growth with $\alpha_{1} \geq 0$, then the function $\lambda \mapsto \operatorname{HD}\left(J_{r}\left(f_{\lambda}\right)\right), \lambda \in \Lambda$, is realanalytic.

Proof. The proof is a direct consequence of Corollary 9.10, Theorem 8.3, which asserts that $\mathrm{P}_{\lambda}\left(\operatorname{HD}\left(J_{r}\left(f_{\lambda}\right)\right)\right)=0$, and the Implicite Function Theorem supported by Theorem 7.14 from which follows that

$$
\frac{\partial}{\partial T} \mathrm{P}_{\lambda}(T)=-\int \log \left|f_{\lambda}^{\prime}\right|_{\tau} d \mu<0
$$

where the differentiation is taken at the point $\left(\lambda, \operatorname{HD}\left(J_{r}\left(f_{\lambda}\right)\right)\right)$ and $\mu$ is the Gibbs (equilibrium) state of the potential $-\operatorname{HD}\left(J_{r}\left(f_{\lambda}\right)\right) \log \left|f_{\lambda}^{\prime}\right|_{\tau}$.

From now on assume that the bounded real-valued harmonic function $h$ is defined on the set $W_{\Lambda}=\bigcup_{\lambda \in \Lambda} B\left(J\left(f_{\lambda}\right), 2 \delta_{f_{\lambda}}\right)$ and $W_{\Lambda}$ is disjoint from the postsingular set of all maps $f_{\lambda}, \lambda \in \Lambda$. So, in particular, our, up to here considerations are independent of the point $\lambda_{0} 1 \Lambda$. In view of Lemma 8.2 and formula (9.9) for every $\lambda \in \Lambda$ and every $q \in \mathbb{R}$ there exists a unique "temperature" value $T_{\lambda}(q) \in \mathbb{R}$ such that $\left(q, T_{\lambda}(q)\right) \in \Sigma_{2}\left(\phi_{\lambda},-\log \left|f_{\lambda}^{\prime}\right|_{\tau}\right) \cap \mathbb{R}^{2}$ such that $\mathrm{P}_{\lambda}\left(q, T_{\lambda}(q)\right)=0$. A direct application of Corollary 9.10 and the Implicite Function Theorem supported by Theorem 7.14, which asserts that

$$
\left.\frac{\partial}{\partial T}\right|_{\lambda, q, T_{\lambda}(q)} \mathrm{P}_{\lambda}(q, T)=-\int \log \left|f_{\lambda}^{\prime}\right|_{\tau} d \mu_{q}<0
$$

( $\mu_{q}$ is the Gibbs (equilibrium) state of the potential $q \phi_{\lambda}-T_{\lambda}(q) \log \left|f_{\lambda}^{\prime}\right|_{\tau}$ ), gives the following.

Corollary 9.12. The temperature function $(\lambda, q) \mapsto T_{\lambda}(q),(\lambda, q) \in \Lambda \times \mathbb{R}$, is real-analytic.

In view of Theorem 8.8, for every $\lambda \in \Lambda$, the range of the function $q \mapsto-T_{\lambda}^{\prime}(q)$, $q \in \mathbb{R}$, is an open interval $\left(\alpha_{1}(\lambda), \alpha_{2}(\lambda)\right.$ with $0 \leq \alpha_{1}(\lambda) \leq \alpha_{2}(\lambda)<+\infty$. As an immediate consequence of Corollary 9.12, we get the following. 
Lemma 9.13. The functions $\lambda \mapsto \alpha_{1}(\lambda)$ and $\lambda \mapsto \alpha_{2}(\lambda), \lambda \in \Lambda$, are respectively upper and lower semi-continuous.

In turn, as an immediate consequence of this lemma, we get the following.

Proposition 9.14. Recall that $\phi_{\lambda}=-t \log \left|f_{\lambda}^{\prime}\right|_{\tau}+h: \bigcup_{\lambda \in \Lambda} B\left(J\left(f_{\lambda}\right), \delta_{f_{\lambda}}\right) \rightarrow \mathbb{R}$. Then the set

$$
U(t, h)=\bigcup_{\lambda \in \Lambda}\{\lambda\} \times\left(\alpha_{1}(\lambda), \alpha_{2}(\lambda)\right) \subset \mathbb{C} \times \mathbb{R}
$$

is open.

Given $\lambda \in \Lambda$, let $\mu_{\lambda}$ be the Gibbs state corresponding to the potential $\phi_{\lambda}$ and the dynamical system $f_{\lambda}: J\left(f_{\lambda}\right) \rightarrow J\left(f_{\lambda}\right)$. We define the function $\mathcal{F}_{\phi}: U_{t, h} \rightarrow[0,2]$ by the formula

$$
\mathcal{F}_{\phi}(\lambda, \alpha)=\mathcal{F}_{\phi_{\mu_{\lambda}}}(\alpha)
$$

The main theorem of this section and, in a sense, a culminating point of the whole paper, is the following.

Theorem 9.15. The function $\mathcal{F}_{\phi}: U_{t, h} \rightarrow \mathbb{R}$ is real-analytic.

Proof. It follows from Theorem 8.8 that for every $(\lambda, q) \in \Lambda \times \mathbb{R}$,

$$
\mathcal{F}_{\phi}\left(\lambda,-T_{\lambda}^{\prime}(q)\right)=T_{\lambda}(q)-q T_{\lambda}^{\prime}(q) .
$$

Now, fix an element $\left(\lambda_{0}, \alpha_{0}\right) \in U_{t, h}$. Then $\alpha_{0} \in\left(\alpha_{1}\left(\lambda_{0}\right), \alpha_{2}\left(\lambda_{0}\right)\right)$ and, in particular, $\alpha_{1}\left(\lambda_{0}\right)<\alpha_{2}\left(\lambda_{0}\right)$. It then follows from Theorem 8.8 that there exists a unique $q_{0} \in \mathbb{R}$ such that $\alpha_{0}=-T_{\lambda_{0}}^{\prime}\left(q_{0}\right)$ and $T_{\lambda_{0}}^{\prime \prime}\left(q_{0}\right) \neq 0$. Therefore, applying the Implicit Function Theorem to the real-analytic function $G(\lambda, \alpha, q)=\alpha+T_{\lambda}^{\prime}(q)$ (see Corollary 9.12), we see that there exists a real-analytic function $\rho: V \rightarrow \mathbb{R}$ defined on an open neighborhood $V \subset U_{t, h}$ of $\left(\lambda_{0}, \alpha_{0}\right)$ such that $\rho\left(\lambda_{0}, \alpha_{0}\right)=q_{0}$ and $\alpha=-T_{\lambda}^{\prime}(\rho(\lambda, \alpha))$ for all $(\lambda, \alpha) \in V$. Hence, $\mathcal{F}_{\phi}(\lambda, \alpha)=T_{\lambda}(\rho(\lambda, \alpha))+\rho(\lambda, \alpha) \alpha$ for all $(\lambda, \alpha) \in V$. Since compositions and products of real-analytic functions are real-analytic, we are done.

We now shall look a little bit closer at the structure of the set $U_{a, \phi}$. We start with the following trivial observation following immediately from its definition.

Proposition 9.16. The set $U_{t, h}$ is vertically connected, i.e. for every $\lambda \in \Lambda$, the set $(\{\lambda\} \times \mathbb{R}) \cap U_{t, h}$ is connected.

The family $\left\{\phi_{\lambda}\right\}_{\lambda \in \Lambda}$ of tame potentials is called essential if for no $\lambda \in \Lambda$, the function $\phi_{\lambda}$ is cohomologous to $-\operatorname{HD}\left(J_{r}\left(f_{\lambda}\right)\right) \log \left|f_{\lambda}^{\prime}\right|_{\tau}$ in the class of all Hölder continuous functions.

Theorem 9.17. If the family $\left\{\phi_{\lambda}\right\}_{\lambda \in \Lambda}$ of tame potential is essential, then the orthogonal projection of $U(t, h)$ on $\mathbb{C}$ is equal to $\Lambda$. If in addition $\Lambda$ is connected, then so is $U(t, h)$. 
Proof. Let $\pi_{1}: \mathbb{C} \times \mathbb{R} \rightarrow \mathbb{R}$ be the projection onto the first coordinate. It is obvious that $\pi_{1}(U(t, h)) \subset \Lambda$. Since family $\left\{\phi_{\lambda}\right\}_{\lambda \in \Lambda}$ is essential it follows from Theorem 8.8 that $\alpha_{1}(\lambda)<\alpha_{2}(\lambda)$ for all $\lambda \in \Lambda$. Consequently $\pi_{1}(U(t, h)) \supset \Lambda$. Now, it follows from Lemma 9.13 that for every $\lambda \in \Lambda$ there exists radius $r(\lambda)>0$ such that the set $D(\lambda, r(\lambda)) \subset \Lambda$ such that $U_{\lambda}:=\bigcup_{\gamma \in D(\lambda, r(\lambda))}\{\gamma\} \times\left(\alpha_{1}(\gamma), \alpha_{2}(\gamma)\right) \subset$ $U(t, h)$ is connected. Suppose now in addition that the set $\Lambda \subset \Lambda$ is connected. Fix two arbitrary points $(\lambda, \alpha),\left(\lambda, \alpha^{\prime}\right) \in U(t, h)$. Then there exists a compact (polygonal) arc $\gamma$ joining $\lambda$ and $\lambda^{\prime}$ in $\Lambda$. The standard compactness argument shows that there exist finitely many points $\lambda_{1}, \lambda_{2}, \ldots, \lambda_{n}$ on $\gamma$ such that $\lambda_{1}=\lambda$, $\lambda_{n}=\lambda^{\prime}$ and $B\left(\lambda_{i}, r\left(\lambda_{i}\right)\right) \cap B\left(\lambda_{i+1}, r\left(\lambda_{i+1}\right)\right) \neq \emptyset$ for all $i=1,2, \ldots, n-1$. Then all the sets $U\left(\phi, B\left(\lambda_{i}, r\left(\lambda_{i}\right)\right)\right) \subset U(t, h), i=1,2, \ldots, n-1$, are connected and

$$
U_{\lambda_{i}} \cap U_{\lambda_{i+1}}=\bigcup\{\lambda\} \times\left(\alpha_{1}(\lambda), \alpha_{2}(\lambda)\right) \neq \emptyset
$$

for all $i=1,2, \ldots, n-1,(\lambda, \alpha) \in U_{\lambda_{1}}$ and $\left(\lambda^{\prime}, \alpha^{\prime}\right) \in U_{\lambda_{n}}$ where the usnion is taken over the set $\left.\phi, B\left(\lambda_{i}, r\left(\lambda_{i}\right)\right) \cap B\left(\lambda_{i+1}, r\left(\lambda_{i+1}\right)\right)\right)$. Hence, the set $U(t, h)$ is connected and we are done.

The next result provides an extremely easy to verify sufficient condition for a harmonic tame potential to be essential. It follows from Theorem 6.20

Proposition 9.18. If $\phi_{\lambda}=-t \log \left|f_{\lambda}^{\prime}\right|_{\tau}+h$ and $t \geq 2$, then the family $\left\{\phi_{\lambda}\right\}_{\lambda \in \Lambda}$ is essential.

Proof. First notice that of $\phi$ and $\psi$ are tame potentials cohomologous modulo constant, then $\kappa(\phi)=\kappa(\psi)$. Since $\kappa\left(-\operatorname{HD}\left(J_{r}\left(f_{\lambda}\right)\right) \log \left|f_{\lambda}^{\prime}\right|\right)=\operatorname{HD}\left(J_{r}\left(f_{\lambda}\right)\right)$ and since $\operatorname{HD}\left(J_{r}\left(f_{\lambda}\right)\right)<2$ for all $\lambda \in H y p$, we are done. 


\section{Bibliography}

[AO] J. Aarts, L. Oversteegen, The geometry of Julia sets, Trans. Amer. Math. Soc. 338 (1993), 897-918.

[Ba] K. Barański, Hausdorff dimension and measures on Julia sets of some meromorphic functions, Fund. Math. 147 (1995), 239-260.

[BD] R. Bhattacharjee, R. Devaney, Tying hairs for structurally stable exponentials, Ergod. Th. Dyn. Sys. 20 (2000),1603-1617

[Bw1] W. Bergweiler, Iteration of meromorphic functions, Bull. A.M.S. 29:2 (1993), 151-188.

[Bw2] W. Bergweiler, Non-real periodic points of entire functions, Canad. Math. Bull. Vol. 40 (3) (1997), 271-275.

[BM] F. Berteloot, V. Mayer, Rudiments de dynamique holomorphe, Cours spécialisés 7, SMF (2000).

[Br] É. Borel, Sur les zéros des fonctions entières, Acta Math. 20 (1897), 357-396.

[Bw1] R. Bowen, R. Bowen, Equilibrium states and the ergodic theory for Anosov diffeomorphisms. LectNotes in Math4்70, Springer (1975).

[Bw2] R. Bowen, Hausdorff dimension of quasi-circles, Publ. Math. IHES, 50 (1980), 11-25.

[CY] W. Cherry, Z. Ye, Nevanlinna's Theory of Value Distribution, Spinger Monographs in Mathematics (2001).

[CS1] I. Coiculescu, B. Skorulski, Thermodynamic formalism of transcendental entire maps of finite singular type, Preprint 2004.

[CS2] I. Coiculescu, B. Skorulski, Perturbations in the Speicer class, Preprint 2004.

[DPU] M. Denker, F. Przytycki, M. Urbański, On the transfer operator for rational functions on the Riemann sphere, Ergod. Th. and Dynam. Sys. 16 (1996), 255-266.

[DU1] M. Denker, M. Urbański, On the existence of conformal measures, Trans. A.M.S. 328 (1991), 563-587.

[DU2] M. Denker, M. Urbański, Ergodic theory of equilibrium states, Nonlinearity 4 (1991), 103134.

[De] R. Devaney, Cantor bouquets, explosions, and Knaster continua: dynamics of complex exponentials. Publ. Mat. 43 (1999), no. 1, 27-54.

[DK] R. Devaney, M. Krych, Dynamics of $\operatorname{Exp}(z)$, Ergod. Th. \& Dynam. Sys. 4 (1984), 35-52.

[Elf] Elfving, G., Über eine Klasse von Riemannschen Flächen und ihre Uniformisierung, Acta Soc. Sci. Fenn. 2 (1934).

[Er] A. Eremenko Ahlfors' contribution to the theory of meromorphic functions .

[EL] A. E. Eremenko, M.Yu. Lyubich, Dynamical properties of some classes of entire functions, Ann. Inst. Fourier, Grenoble 42, 4 (1992), 989-1020.

[Go] M. I. Gordin, The Central Limit Theorem for stationary processes, Soviet Math. Dokl., 10 N. 5 (1969), 1174-1176.

[Hy] W. K. Hayman, Meromorphic functions, Oxford, Clarendon Press (1964).

[Hk] J. M. Hemke, Measurable dynamics of meromorphic maps, thesis, Kiel (2005).

[H1] E. Hille Analytic function theory, Vol. II, Ginn (1962).

[H2] E. Hille Ordinary differential equations in the complex domain, Dover Publications (1997).

[H3] E. Hille On the zeroes of the Functions of the Parabolic Cylinder, Ark. Mat. Astron. Fys., Vol. 18, No. 26 (1924).

[Hk] A. Hinkkanen, A sharp form of Nevanlinna's second fundamental theorem, Invent. Math. 108 (1992), 549-574.

[IL] I. A. Ibragimov, Y. V. Linnik, Independent and stationary sequences of random variables. Wolters-Noordhoff Publ., Groningen 1971. 
[IM] C. Ionescu-Tulcea, G. Marinescu, Théorie ergodique pour des classes d'operations noncomplètement continues, Ann. Math. 52, (1950), 140-147.

[Iv] F. Iversen Recherches sur les fonctions inverses dess fonctions méromorphes, Thèse de Helsingfors (1914).

[JV] G. Jank, L. Volkmann, Meromorphe Funktionen und Differentialgleichungen,

[Ka] T. Kato, Perturbation theory for linear operators, Springer (1995).

[KU1] J. Kotus, M. Urbański, Conformal, geometric and invariant measures for transcendental expanding functions, Math. Annalen. 324 (2002), 619-656.

[KU2] J. Kotus, M. Urbański, Geometry and ergodic theory of non-recurrent elliptic functions, J. d'Analyse Math. 93 (2004), 35-102.

[KU3] J. Kotus, M. Urbański, The dynamics and geometry of the Fatou functions, Preprint 2004, Discrete \& Continuous Dyn. Sys. 13 (2005), 291-338.

[KU4] J. Kotus, M. Urbański, Fractal Measures and Ergodic Theory of Transcendental Meromorphic Functions, Preprint 2004.

[KFS] I. P. Kornfeld, S. V. Fomin, Yakov G. Sinai, Ergodic Theory, Springer (1982).

[Ll] J.K. Langley, Postgraduate notes on complex analysis, preprint.

[Liv] C. Liverani, Central limit theorem for deterministic systems, Pitman Res. Notes Math. Ser., v.362, Longman, Harlow, (1996), 56-75.

[L1] M. Yu. Lyubich, Some typical properties of the dynamics of rational maps, Russian Math. Surveys, 8, 5 (1983) 154-155.

[L2] M. Yu. Lyubich, The dynamics of rational transforms: the topological picture, Russian Math. Surveys, 41, 4 (1986) 43-117.

[MSS] R. Mañé, P. Sad, D. Sullivan, On the dynamics of rational maps, Ann. Scient. Ec. Norm. Sup. 4e série, 16 (1983), 193-217.

[McM] C. McMullen, Area and Hausdorff dimension of Julia set of entire functions, Trans. A.M.S. 300 (1987), 329-342.

[Mane] R. Mane, Ergodic Theory and Differentiable Dynamics, Springer (1987).

[Mat] P. Mattila, Geometry of sets and measures in euclidean spaces, Cambridge Studies in Advanced Mathematics 44, Cambridge University Press, 1995.

[MdU] D. Mauldin and M. Urbański, Dimensions and measures in infinite iterated function systems, Proc. London Math. Soc. (3) 73 (1996) 105-154.

[MU] D. Mauldin, M. Urbański, Graph Directed Markov Systems, Cambridge Univ. Press 2003.

[My1] V. Mayer, Comparing measures and invariant line fields, Ergodic Theory \& Dynamical Syst. $22(2002), 555-570$

[My2] V. Mayer, Rational functions without conformal measures on the conical set, Preprint 2002.

[My3] V. Mayer, The size of the Julia set of meromorphic functions, Math. Nachrichten (to appear).

[MyU1] V. Mayer, M. Urbański, Gibbs and equilibrium measures for elliptic functions, Math. Zeitschrift 250 (2005), 657-683.

[MyU2] V. Mayer, M. Urbański, Geometric Thermodynamical Formalism and Real Analyticity for Meromorphic Functions of Finite Order, Ergod. Th. \& Dynam. Sys. 28 (2008), 915-946

[MyU3] V. Mayer, M. Urbański, Fractal Measures for Meromorphic Functions of Finite Order, Dynam. Sys. 22 (2007), 169178.

[Nev1] R. Nevanlinna, Eindeutige Analytische Funktionen, Springer Verlag, Berlin (1953).

[Nev2] R. Nevanlinna, Analytic Functions, Springer Verlag, Berlin (1970).

[Nev3] R. Nevanlinna, Über Riemannsche Flächen mit endlich vielen Windungspunkten, Acta Math. 58 (1932), 295-373.

[Py] W. Parry, Entropy and Generators in Ergodic Theory, New York, W. A. Benjamin (1969).

[Pa] S. J. Patterson, The limit set of a Fuchsian group, Acta Math. 136 (1976), 241-273.

[PU] F. Przytycki, M. Urbański, Fractals in the Plane - the Ergodic Theory Methods, available on Urbański's webpage, to appear Cambridge Univ. Press.

[PUZ] F. Przytycki, M. Urbański, A. Zdunik, Harmonic, Gibbs and Hausdorff measures on repellers for holomorphic maps I, Ann. of Math. 130 (1989), 1-40.

[ReSi] M. Reed, B. Simon Methods of Modern Mathematical Physics, IV: Analysis of Operators, (1978) Academic Press.

[Rem] L. Rempe, Hyperbolic dimension and reduced Julia sets of transcendental functions, Proc. Amer. Math. Soc. (to appear, published online Nov. 2008). 
[RiSt] P.J. Rippon, G.M. Stallard, Iteration of a class of hyperbolic meromorphic functions, Proc. of the AMS, Vol. 127, Nr. 11 (1999), 3251-3258.

[Ro] R. T. Rockafellar, Convex analysis, (1970) Princeton University Press.

[Ru] W. Rudin, Functional Analysis, (1991) McGraw-Hill, Inc.

[R1] D. Ruelle, Thermodynamic Formalism, (1978) Adison-Wesley.

[R2] D. Ruelle, Repellers for real analytic maps, Ergod. Th. \& Dynam. Sys., 2 (1982), 99-107.

[Sch] H. Schaefer, Banach lattices and positive operators, (1974) Springer.

[Sk] Z. Slodkowski, Holomorphic motions and polynomial hulls, Proc. Amer. Math. Soc. 111 (1991), 347-355

[St1] G.M. Stallard, The Hausdorff dimension of Julia sets of hyperbolic meromorphic functions, Math. Proc. Cambridge Philos. Soc. 127 (1999), no. 2, 271-288.

[St2] G.M. Stallard, Dimension of Julia sets of transcendental meromorphic functions, preprint.

[Su] D. Sullivan, Seminar on conformal and hyperbolic geometry, Preprint IHES (1982).

[Ur1] M. Urbański, Measures and dimensions in conformal dynamics, Bull. Amer. Math. Soc. 40 (2003), 281-321.

[Ur2] M. Urbański, Recurrence Rates for Loosely Markov Dynamical Systems, Journal of Australian Math. Soc. 82 (2007), 39-57.

[UZ1] M. Urbański, A. Zdunik, The finer geometry and dynamics of exponential family, Michigan Math. J. 51 (2003), 227-250.

[UZ2] M. Urbański, A. Zdunik, Real analyticity of Hausdorff dimension of finer Julia sets of exponential family, Ergod. Th. \& Dynam. Sys. 24 (2004), 279-315.

[UZ3] M. Urbański, A. Zdunik, Geometry and ergodic theory of non-hyperbolic exponential maps, Preprint 2003, to appear Trans. Amer. Math. Soc.

[UZ4] M. Urbański, A. Zdunik, Maximizing Measures on Metrizable Non-Compact Spaces, Preprint 2004, to appear Math. Proc. Edinburgh Math. Soc.

[UZi] M. Urbański, M. Zinsmeister, Geometry of hyperbolic Julia-Lavaurs sets, Indagationes Math. 12(2), (2001), 273-292

[Zd] A. Zdunik, Parabolic orbifolds and the dimension of the maximal measure for rational maps, Invent. Math. 99 (1990), no. 3, pp.627-649.

[Zin] M. Zinsmeister, Formalisme Thermodynamique et systèmes dynamiques holomorphes, Panoramas et Synthèses, N.4, SMF (1996). 